\title{
The ATLAS inner detector trigger performance in pp collisions at 13 TeV during LHC Run 2
}

\author{
ATLAS Collaboration ${ }^{\star}$ \\ CERN, 1211 Geneva 23, Switzerland
}

Received: 6 July 2021 / Accepted: 7 December 2021 / Published online: 8 March 2022

(C) CERN for the benefit of the ATLAS collaboration 2022

\begin{abstract}
The design and performance of the inner detector trigger for the high level trigger of the ATLAS experiment at the Large Hadron Collider during the 2016-2018 data taking period is discussed. In 2016, 2017, and 2018 the ATLAS detector recorded $35.6 \mathrm{fb}^{-1}, 46.9 \mathrm{fb}^{-1}$, and $60.6 \mathrm{fb}^{-1}$ respectively of proton-proton collision data at a centre-of-mass energy of $13 \mathrm{TeV}$. In order to deal with the very high interaction multiplicities per bunch crossing expected with the $13 \mathrm{TeV}$ collisions the inner detector trigger was redesigned during the long shutdown of the Large Hadron Collider from 2013 until 2015. An overview of these developments is provided and the performance of the tracking in the trigger for the muon, electron, tau and $b$-jet signatures is discussed. The high performance of the inner detector trigger with these extreme interaction multiplicities demonstrates how the inner detector tracking continues to lie at the heart of the trigger performance and is essential in enabling the ATLAS physics programme.
\end{abstract}

\section{Contents}

1 Introduction .................. 1

2 The ATLAS detector, trigger and data acquisition systems . . . . . . . . . . . . . . 2

3 The inner detector trigger $\ldots \ldots \ldots$. . . . . . . . 4

3.1 The inner detector trigger for Run $1 \quad \ldots \ldots .4$

3.2 Evolution of the inner detector trigger strategy from Run 1 to Run 2 . . . . . . . . . . . . . 4

3.3 The inner detector trigger in Run $2 \ldots \ldots .6$ 3.3.1 Data preparation . . . . . . . . . 7

3.3.2 Fast tracking ........... . . 8

3.3.3 Precision tracking . . . . . . . . . . . 9

3.3.4 Vertex reconstruction . . . . . . . . . 9 9

3.4 Multistage tracking . . . . . . . . . . . . 9 9

4 Inner detector trigger timing . . . . . . . . . . 11

4.1 Algorithm execution time in the muon trigger . 12

4.2 Algorithm execution time in the electron trigger 13

^e-mail: atlas.publications@cern.ch
4.3 Algorithm execution time in the tau trigger . . 13

4.4 Algorithm execution time in the $b$-jet trigger and vertexing . . . . . . . . . . . 13

4.5 Total tracking time per event . . . . . . . . 15

5 High-level trigger tracking performance . . . . . . . 15

5.1 Data selection . . . . . . . . . . . 15

5.2 Track selection . . . . . . . . . . 16

5.3 Tracking in the muon signature . . . . . . . 17

5.4 Tracking in the electron signature . . . . . . 21

5.5 Tracking in the tau signature . . . . . . 30

5.6 Tracking in the $b$-jet signature . . . . . . 31

5.7 Vertex finding performance . . . . . . . . . 36

6 Conclusion .................. 41

References . . . . . . . . . . . . . . . . . . 42

\section{Introduction}

The trigger system is an essential component of any collider experiment - especially so for a hadron collider - as it is responsible for deciding whether or not to keep an event from a given bunch-crossing interaction for later study. During Run 1 (2009 to early 2013) of the Large Hadron Collider (LHC) [1], the ATLAS detector [2] trigger system [3-10] operated efficiently at instantaneous luminosities of up to $8 \times 10^{33} \mathrm{~cm}^{-2} \mathrm{~s}^{-1}$ and primarily at centre-of-mass energies, $\sqrt{s}$, of $7 \mathrm{TeV}$ and $8 \mathrm{TeV}$. In Run $2(2015-18)$ the increased centre-of-mass energy of $13 \mathrm{TeV}$, higher luminosity and increased number of proton-proton interactions per bunchcrossing (pile-up) required a significant upgrade to the trigger system. This was necessary to avoid the required processing time exceeding that available when running with the trigger thresholds that are needed to satisfy the ATLAS physics programme. For this reason, the first long shutdown between LHC Run 1 and Run 2 was used to improve the trigger system with almost no component left untouched.

Final states involving electrons, muons, taus, and $b$-jets [8,11-15], provide important signatures for many precision measurements of the Standard Model and searches for new physics. The ability of the ATLAS trigger to process infor- 
mation from the inner detector (ID) [16] to reconstruct particle trajectories is an essential requirement for the efficient triggering of these objects at manageable rates. Typical rate rejection factors possible using tracking information from the ID trigger range very approximately from 50 to 100 for the electron and $b$-jet triggers, and from 3 to 10 for the muon and tau triggers. Without this rate rejection from the ID trigger it would not be possible to achieve the goals of the ATLAS physics programme. The ID trigger must therefore be able to reconstruct tracks with high efficiency across the entire range of possible physics signatures, under all expected circumstances. This challenge is exacerbated by the extremely high track and hit occupancies in the ID that arise for the large pile-up multiplicity present during LHC running at the highest operation intensities.

The performance of the ATLAS trigger system for the lower luminosity running in 2015 has been reported previously [4], and included a brief description of the ID trigger design. In this paper the full details of the ID trigger design and implementation are presented, followed by a discussion of the execution time of the different components of the ID trigger and the full performance in terms of efficiency and resolution from the 2016-2018 running period.

\section{The ATLAS detector, trigger and data acquisition systems}

The ATLAS detector [2] at the LHC is a multi-purpose particle detector with a forward-backward symmetric cylindrical geometry and a near $4 \pi$ coverage in solid angle around the collision point. ${ }^{1}$ It consists of an inner tracking detector surrounded by a thin superconducting solenoid, providing a $2 \mathrm{~T}$ axial magnetic field, electromagnetic and hadronic calorimeters, and a muon spectrometer incorporating large superconducting toroidal magnets. Owing to the cylindrical geometry, subdetector components generally consist of a barrel region with central pseudorapidities close to zero, and two endcap components, one each in the forward regions with large absolute pseudorapidity.

The inner detector, shown schematically in Fig. 1, allows the precise reconstruction of charged-particle trajectories in the pseudorapidity range $|\eta|<2.5$. It consists of three subsystems: the high granularity silicon pixel detector, the silicon microstrip tracker, and the transition radiation tracker.

\footnotetext{
1 ATLAS uses a right-handed coordinate system with the origin at the nominal interaction point (IP) at the centre of the detector and the $z$-axis along the beam direction; the $x$-axis pointing from the IP to the centre of the LHC ring, and the $y$-axis pointing upwards. Cylindrical coordinates $(r, \phi)$ are used in the transverse plane, $\phi$ being the azimuthal angle around the $z$-axis. The pseudorapidity is defined in terms of the polar angle, $\theta$, as $\eta=-\ln \tan (\theta / 2)$. Angular distance is measured using $\Delta R \equiv \sqrt{(\Delta \eta)^{2}+(\Delta \phi)^{2}}$
}

The pixel detector consists of three barrel layers, and three layers in each endcap, and an insertable B-layer (IBL) [17,18] closest to the interaction point, installed before Run 2. The IBL spans the entire pseudorapidity range of the inner detector. The pixel detector typically provides four measurements per track. The pixel detector is surrounded by the silicon microstrip tracker (SCT) $[19,20]$, which consists of double layer silicon strip detectors, four in the barrel, and nine in each endcap. These provide axial hits, or azimuthal hits in the endcaps, and small-angle stereo hits used to provide improved spatial resolution in the longitudinal direction along the modules. The SCT typically provides eight measurements per track. The silicon detectors are complemented by the transition radiation tracker (TRT) [21-23], consisting of multiple layers of straw cylindrical drift tubes filled with either a Xe$\mathrm{CO}_{2}-\mathrm{O}_{2}$, or $\mathrm{Ar}-\mathrm{CO}_{2}-\mathrm{O}_{2}$ mixture, and which enables radially extended track reconstruction up to $|\eta|=2.0$.

The calorimeter system covers the pseudorapidity range $|\eta|<4.9$. Within the region $|\eta|<3.2$, electromagnetic calorimetry is provided by barrel and endcap high granularity lead/liquid-argon (LAr) calorimeters, with an additional thin LAr presampler covering $|\eta|<1.8$ to correct for energy loss in material inside the calorimeter radius. Hadronic calorimetry is provided by the steel/scintillator-tile calorimeter, and two copper/LAr hadronic endcap calorimeters. The more forward regions have copper/LAr and tungsten/LAr calorimeter modules optimised for electromagnetic and hadronic measurements respectively.

The muon spectrometer (MS) [24] is composed of separate trigger and high-precision tracking chambers measuring the deflection of muons in a magnetic field generated by superconducting air-core toroids. The field integral of the toroids ranges between $2.0 \mathrm{Tm}$ and $6.0 \mathrm{Tm}$ across most of the detector. Precision chambers cover the region $|\eta|<2.7$ and are complemented by cathode-strip chambers in the forward region. The muon trigger system $[3,4,25]$ covers the range $|\eta|<2.4$ with resistive-plate chambers in the barrel, and thin-gap chambers in the endcap regions.

Interesting events are selected to be recorded by the first level trigger system (Level 1, L1) [3,26] implemented in custom hardware, followed by selections made by algorithms implemented in software in the high level trigger (HLT) $[3,4]$. The $\mathrm{L} 1$ trigger processes events at the $40 \mathrm{MHz}$ bunch crossing rate. Events are accepted at a rate below $100 \mathrm{kHz}$, which the HLT further reduces in order to record events to permanent storage at approximately $1.2 \mathrm{kHz}$ [27].

The ATLAS trigger and data acquisition (TDAQ) system used during Run 1 [3,28] consisted of a multi-level trigger system with a hardware, pipelined Level 1 trigger and two large farms of commodity CPUs, one for the Level 2 (L2) software trigger, and the second for the third level, event filter (EF) processing. In Run 1 the separate L2 and EF farms collectively constituted the HLT system. For the 


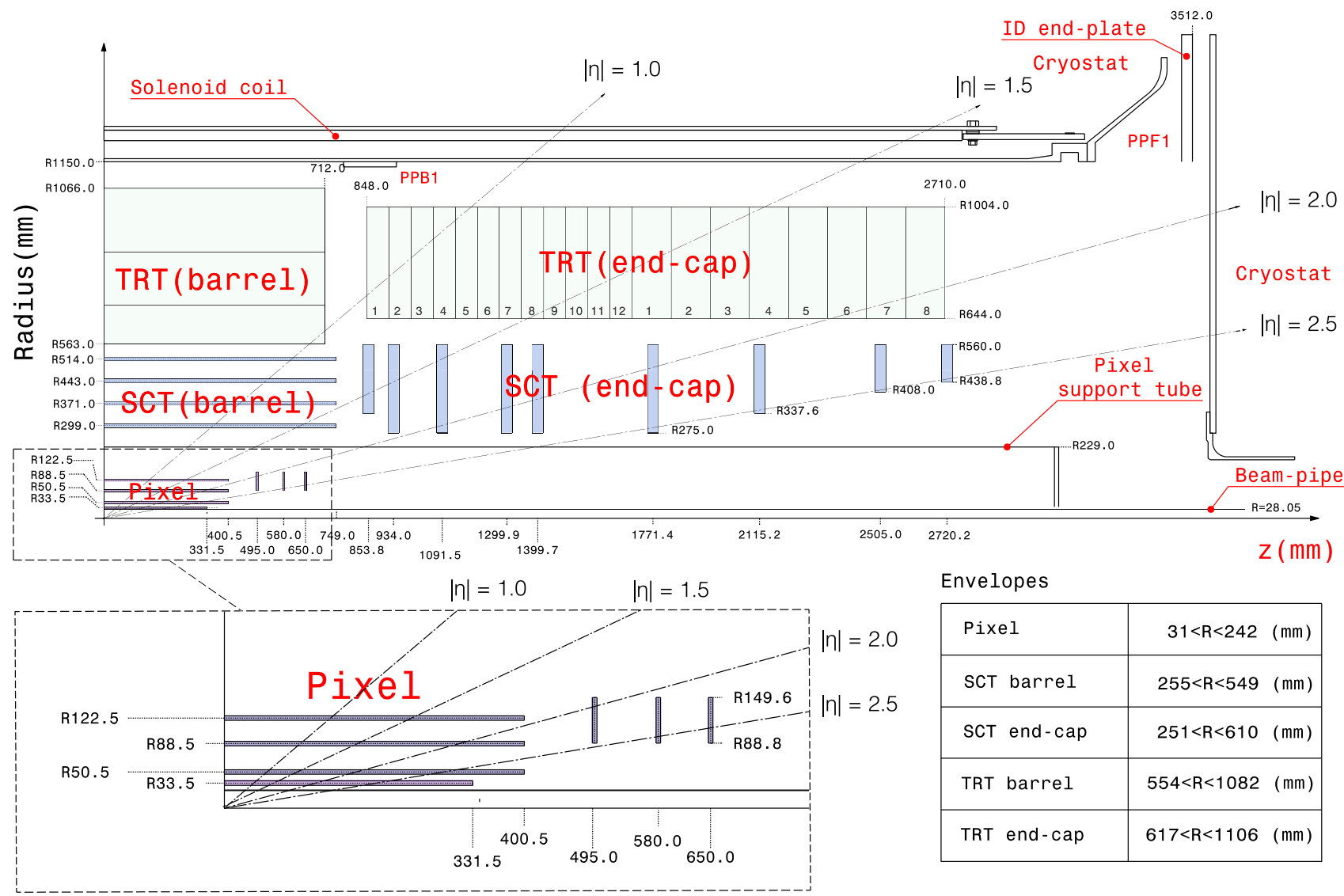

Fig. 1 A cross section of one quadrant of the ATLAS inner detector, showing the pixel, SCT and TRT barrel and endcap sections. The pixel detector includes the insertable b-layer

L1 trigger operation, a reduced granularity subsample of the data from the calorimeters and the muon spectrometer are read out and processed in a custom read-out path and sent to the off-detector hardware L1 processors. In parallel, the data from the entire detector are stored in custom on-detector pipelined read-out buffers (ROBs) [29] ready to be read out in the eventuality of a L1 trigger accept decision. Upon such a decision, the pipelines are stopped and the data for the corresponding bunch crossing is read out from the ROBs to the off-detector read-out subsystem, ready for distribution to the farm of the HLT processing nodes. Because of the extremely high data volume in the SCT and pixel detectors, these can be read out only following an L1 accept and therefore the first stage at which tracks could be reconstructed in the silicon layers during Run 1 was at Level 2. The coarse granularity muon spectrometer or calorimeter data read out for the L1 trigger are used to reconstruct objects of interest, such as potential track segments in the muon spectrometer from muon candidates, or high energy clusters reconstructed in the calorimeter. These objects are used to define regions of interest (RoI) in the detector which are worth reconstructing with the data read out at the full granularity. Using RoI typically reduces the amount of data to be transferred and processed in the HLT to between $2 \%-6 \%$ of the total data volume [6].

For Run 2, the LHC increased the centre-of-mass energy in $p p$ collisions from 8 to $13 \mathrm{TeV}$, with a reduction of the nominal bunch spacing from 50 to $25 \mathrm{~ns}$ [30] and an increase in the beam intensity per bunch crossing. This increase lead to a significant increase in the mean pile-up interaction multiplicity per bunch crossing, with correspondingly higher track and hit multiplicities.

The increase in beam energies meant that the trigger rates were on average a factor of 2-2.5 times larger for the same luminosity and with the same trigger criteria [4]. In order to prepare for the significantly higher expected rates in Run 2, several trigger upgrades were implemented during the 20132015 long shutdown, which are summarised here.

For Run 2, the separate L2 and EF farms were combined into a single, homogeneous HLT farm [31]. During Run 1, Level 2 requested only partial event data to be sent over the network, while the event filter operated on the full event information assembled by separate farm nodes dedicated to event building [32]. For Run 2, merging the separate farms into a single homogeneous farm allowed for better resource sharing and an overall simplification of both the hardware and 
software. To achieve higher read-out and output rates, the read-out subsystem, data collection network, and data storage system were upgraded [33]. The on-detector front-end electronics and detector-specific read-out systems were not changed for Run 2 operation in any significant way.

During Run 2, the typical mean processing time for the complete HLT processing per event was between 0.4 and $0.8 \mathrm{~s}$ for the higher pile-up at the start of a fill, decreasing to around $200 \mathrm{~ms}$ for lower pile-up.

\section{The inner detector trigger}

The HLT is the first level where information from the silicon detectors in the ID is available in the trigger. The track reconstruction in the HLT is then performed either within an RoI identified at L1 or for the full detector. Throughout Run 1 and Run 2, the HLT ran progressively more complex algorithms to reduce the rate of events to be processed successively throughout the HLT. Starting from the initial input rate determined by the L1 trigger accept rate, the fastest algorithms were executed first to reduce that rate at which these more complicated algorithms needed to run. To achieve this, the reconstruction in the HLT generally consists of a two-stage approach with a fast track reconstruction stage to reject the less interesting events, followed by a slower, precision reconstruction stage - the precision tracking - for those events remaining after the fast reconstruction. For both Run 1 and Run 2, each of the initial RoI used in the HLT is wedge shaped, extending in $z$ along the full $450 \mathrm{~mm}$ interaction region at the beam line and opening out in $\eta$ and $\phi$. This large extent in $z$ is required since the $z$ position of the interaction is not known before the tracking itself has been executed.

During the 2013-15 LHC long shutdown, many changes were implemented in the ID trigger to better handle the changing run conditions expected in Run 2. To motivate these changes, Sect. 3.1 briefly summarises the tracking strategy used during Run 1 and is followed in Sect. 3.2 by an explanation of the developments implemented for Run 2 and why they were needed. The subsequent sections describe in detail the structure and algorithms used in the ID trigger during Run 2.

\subsection{The inner detector trigger for Run 1}

For Run 1 the ID tracking in the trigger $[3,34,35]$ ran the fast reconstruction stage on the dedicated L2 CPU farm and the precision tracking stage on the dedicated Event Filter CPU farm. This division meant that the information passed between the different trigger levels was essentially limited to the RoI within which the reconstruction should run, necessitating that all stages in the track reconstruction, including

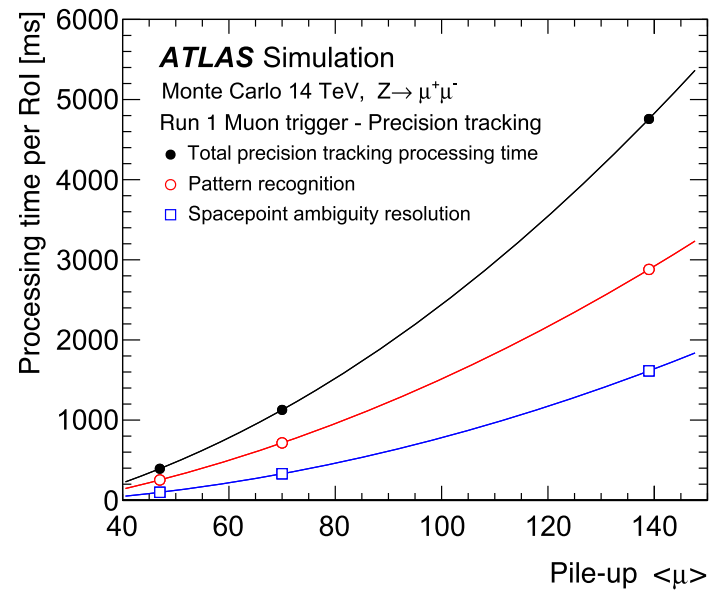

Fig. 2 The total processing time for the Run 1 precision tracking timing as a function of pile-up interaction multiplicity

any data preparation, be performed independently in each of the two trigger levels.

At L2 the tracking consisted of several alternative custom pattern recognition algorithms and tracking algorithms, which were all preceded by a fast data preparation stage to read out any data from the pixel detector and SCT, and prepare the space-points. To optimise the tracking efficiency, the best-performing pattern recognition algorithm was chosen independently for each signature. Following the pattern recognition, track candidates were then identified using a fast Kalman filter track fit [36] and the tracks could be extended into the TRT.

In the event filter, the precision tracking stage (referred to as EFID during Run 1) ran offline tracking algorithms adapted for use in the trigger. Starting with the offline data preparation, the tracking continued with the offline pattern recognition, followed by the ambiguity solver [37] which resolves any ambiguity with respect to duplicated hits or hits falsely attributed to tracks, ranking the tracks and rejecting those where the track quality is deemed insufficient. A more detailed exposition of the ID trigger used during Run 1 can be found elsewhere [35].

\subsection{Evolution of the inner detector trigger strategy from Run 1 to Run 2}

The general purpose in running the event filter during Run 1 was to confirm the objects reconstructed and selected by Level 2, and to improve on the precision of their reconstruction, so allowing for the application of tighter selection to further reduce the rate. For the Run 1 trigger, the events processed by the event filter were necessarily required to have passed the Level 2 selection and consequently, running any algorithm in the event filter that was more efficient than its Level 2 counterpart was in some sense unnecessary, as any 


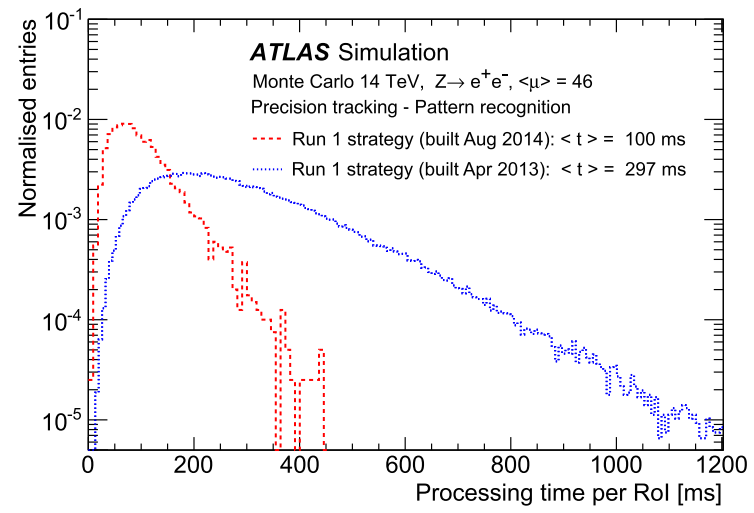

(a)

Fig. 3 The processing time for the Run 1 precision tracking pattern recognition stage (a), and the processing time of the ambiguity solver stage from the Run 1 precision tracking (b). In both cases the process-

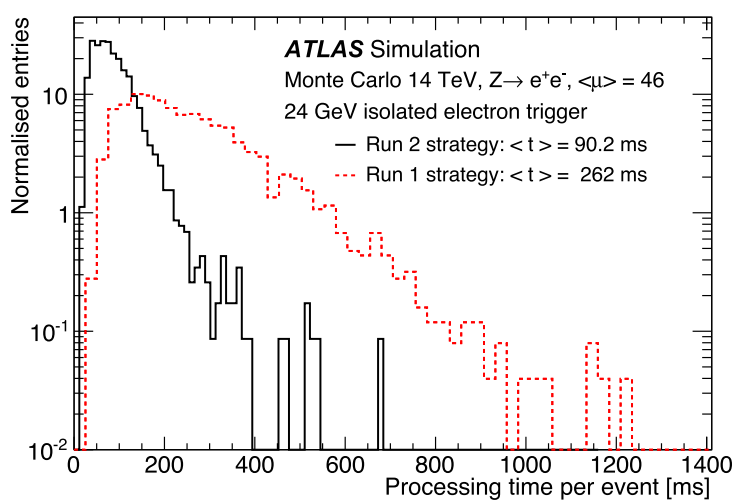

(a)

Fig. 4 The reduction in the latency for the Run 2 ID trigger strategy with respect to the Run 1 strategy: a the total time for the isolated $24 \mathrm{GeV}$ electron trigger with the Run 1 and Run 2 strategies; $\mathbf{b}$ the time taken

event that had not passed the Level 2 stage would have already been discarded. However, the longer processing times available for the offline algorithms executed in the Run 1 event filter afforded by the lower processing rate meant that the EF tracking was indeed more efficient than that in Level 2, but that this additional efficiency could not be exploited as the events would have already been rejected by the Level 2 trigger.

As mentioned in Sect. 2, for Run 2, the previously separate $\mathrm{L} 2$ and EF processing stages were merged to instead run as part of the same process on individual HLT nodes within the HLT farm. This merging allows information from algorithms that previously ran in the $\mathrm{L} 2$ reconstruction stage to more easily be used later in the processing chain, thus removing any need to run separate, duplicated data preparation and pattern recognition stages for the tracking.

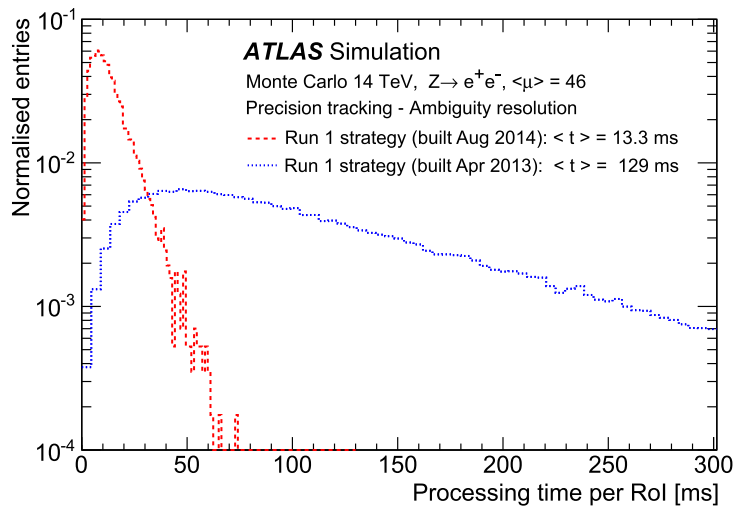

(b)

ing time is shown before and after the improvements to the algorithms implemented during the 2013-2015 long shutdown

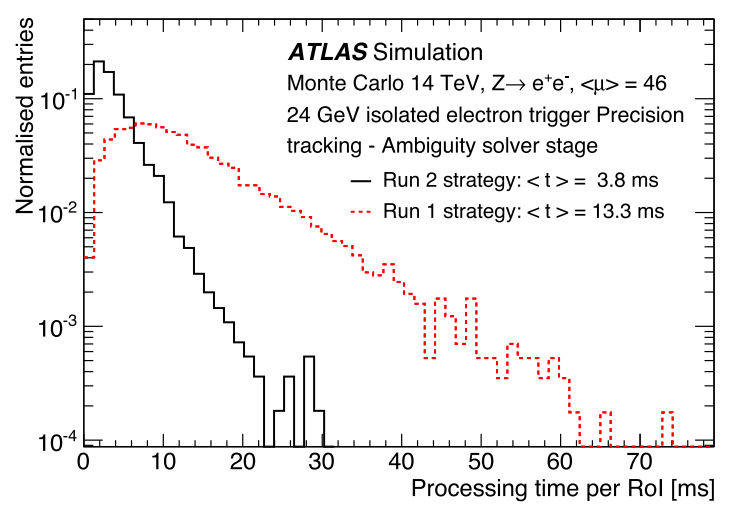

(b)

by the ambiguity solver in the Run 1 and Run 2 strategies. In all cases, the improved offline code implemented late in 2014 has been used in the precision tracking stage

The performance of the offline tracking algorithms as used in the precision tracking for the EF trigger in Run 1 was studied in detail under Run 2 conditions to determine which aspects of the offline tracking could be retained for the ID trigger in Run 2. Figure 2 shows the time taken by the different stages of the EF tracking from the Run 1 ATLAS muon trigger as a function of the mean pile-up interaction multiplicity, $\langle\mu\rangle$, expected during Run 2 and beyond, using simulated $Z \rightarrow \mu^{+} \mu^{-}$events with a $14 \mathrm{TeV}$ centre-of-mass energy. ${ }^{2}$ Under these Run 2 conditions, the pattern recognition stage of the offline tracking can be seen to constitute more than $60 \%$ of the total time of the Run 1 precision tracking with a pile-up of 70 interactions per bunch crossing and does not scale linearly with the pile-up multiplicity.

\footnotetext{
2 The sample was generated before the limitations on the LHC operating energy were fully understood.
} 


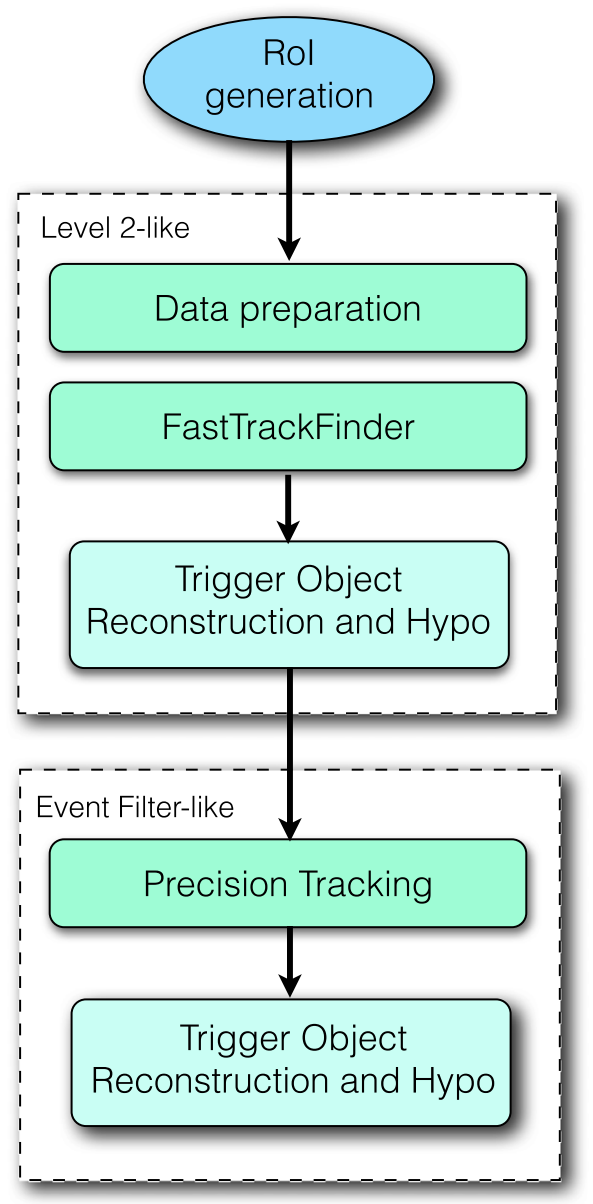

Fig. 5 Schematic illustrating the single-stage tracking for a single RoI. Here single-stage refers to the single processing of a specific RoI, rather than the separate steps in the track processing itself. Selection can be applied in hypothesis algorithms (hypos) which reduce the rate for processing the later steps

In addition to the constraints on the trigger system in Run 2, there are strong constraints on the time taken to perform the offline reconstruction. For this reason an extensive programme of software optimisation $[38,39]$ was undertaken between Run 1 and Run 2. This provided tangible benefits for the ID trigger for those algorithms shared with the offline reconstruction. Improvements in the execution time resulted from improvements to the computing infrastructure - namely the switch to running on a 64-bit, rather than a 32-bit kernel, and using a newer compiler: GCC 4.8 rather than 4.3 - and the replacement of the CLHEP [40] linear algebra library, by the Eigen library [41].

These consequently led to a reduction in the latency of both the pattern recognition and track fitting stages for offline tracking used in the Run 1 precision tracking. The effect on the processing times following these improvements for their modified use in the trigger can be seen in Fig. 3. This shows a clear improvement in the Run 1 pattern recognition strategy and ambiguity solver processing times by factors of approximately three and ten respectively, when running on simulated $Z \rightarrow e^{+} e^{-}$events.

Despite this significant improvement in processing time, the offline pattern recognition was still too time consuming to be used in the trigger with the increase in the pile-up multiplicity expected for Run 2 . As a consequence, the chosen strategy for the ID trigger for Run 2 was to reuse the output from the pattern recognition for the fast tracking by using the identified tracks and hits to seed the ambiguity solver directly, so completely removing the need to run the offline pattern recognition stage in the trigger.

The result of the improvement can be seen in Fig. 4 which shows, for the same simulated $Z \rightarrow e^{+} e^{-}$events, the total time required for the full $24 \mathrm{GeV}$ isolated electron trigger including the calorimeter reconstruction - in this case comparing the Run 2 strategy described above, with that from Run 1, but with the upgraded offline tracking algorithms.

Even with the complete reconstruction of the signature, including the time to run the calorimeter and the space-point reconstruction, the Run 2 strategy is still approximately three times faster than the Run 1 strategy.

Also illustrated in Fig. 4 is the execution time for the improved ambiguity solver code with both the Run 1 and Run 2 strategies. The Run 2 strategy is seen to be approximately three times faster than that used in Run 1 . This is a consequence of the track selection and reduction having been already performed by the fast tracking so that the ambiguity solver is not required to perform as much computation as it would if running on the greater number of track candidates that would arise from the offline pattern recognition.

\subsection{The inner detector trigger in Run 2}

For Run 2, the division of the tracking into fast tracking and precision tracking stages was retained: the fast tracking stage consisting of a newly implemented, trigger-specific pattern recognition stage, based on a hybrid of those used at L2 during Run 1, and the precision tracking stage again relying heavily on offline tracking algorithms, but seeded with information from the fast tracking stage $[4,16]$. As in Run 1, the inner detector data preparation reconstructs clusters, and space-points from the silicon clusters, using the information from the pixels and the SCT strips [42]. The clusters and spacepoints are used for the remainder of the track reconstruction. Since the fast and precision tracking steps were both running as aspects of the same process on single CPU nodes, they can share a single silicon data preparation step.

The generic Run 2 tracking strategy is shown schematically in Fig. 5. This illustrates how the tracking is split into separate steps, which can be separated by additional nontracking-related algorithms to reconstruct additional features, and hypothesis algorithms to select objects which sat- 


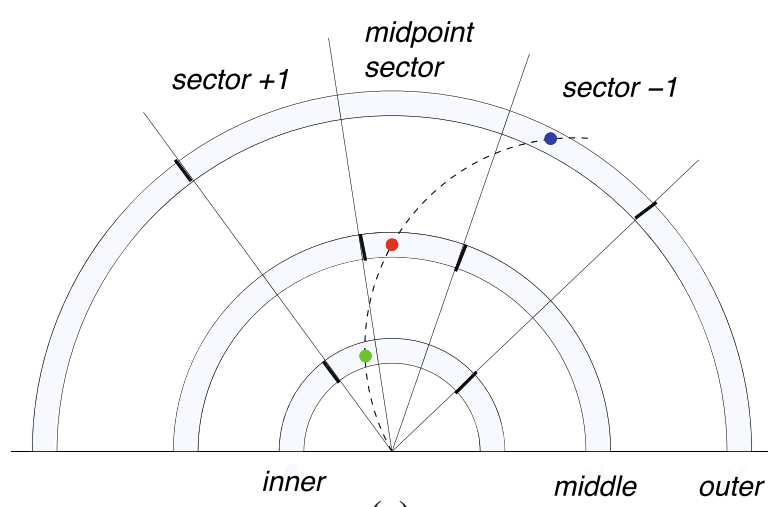

(a)

Fig. 6 Schematic illustrating a the track seed formation in radial bins and azimuthal sectors, where the midpoint sector for a triplet is the sector containing the midpoint, and where the inner and outermost hits are allowed to originate in the same sector, or the the adjacent sectors, and

isfy certain selection criteria. These are used to reduce the rate of RoI to be processed between the fast track finder and precision tracking steps.

\subsubsection{Data preparation}

The pixel and SCT data preparation consists of decoding hits in the silicon modules of the inner detector from a byte stream format, grouping these hits into clusters [42] - necessary because particles deposit charge in several adjacent silicon cells - and forming space-points from the clusters. These represent points in three-dimensional space which are used for the pattern recognition, the constituent clusters themselves being used for the actual track fitting.

The data preparation employs the RoI mechanism, described later in this section, which allows the trigger to request only the data from those silicon modules falling within an RoI, saving both processing time, and data transfer bandwidth [43]. Following the L1 accept the data for each detector are read out to the ROB system, but to reduce the bandwidth only those ROBs containing data corresponding to an RoI need be interrogated for read-out to the HLT.

The computational complexity of the decoding algorithms for both the pixel and SCT detector subsystems is linear with respect to the number of data words, containing the encoded detector data from the subsystem. The decoders iterate over ROB data fragments, and over the data words within each fragment. While looping through an individual fragment, the algorithm maintains information regarding which module it is decoding, assigning raw data objects (RDOs) - in this case, decoded hits - to an appropriate in-memory container. Error information can also be encoded in the byte stream, but this is a fringe case which is not discussed here. Once the data words have been decoded and RDOs created and mapped to their appropriate modules, the clustering algorithm takes

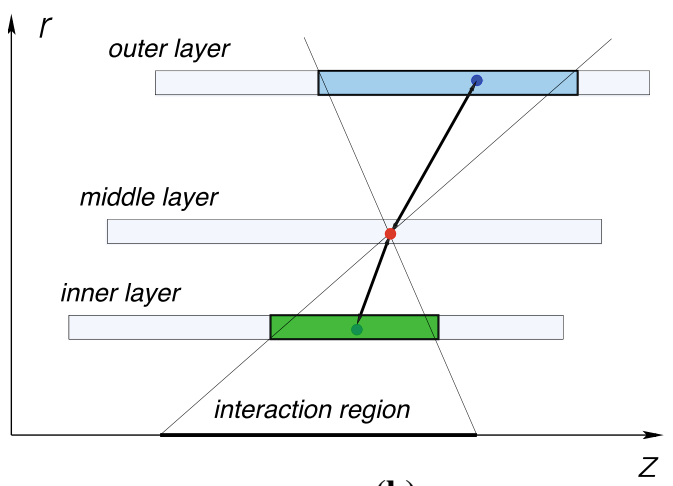

(b)

b track seed formation in the $r-z$ plane, where the extrapolated $z$ position of the triplet at the beamline can be restricted to within a specific $z$ region

over, grouping adjacent hits within a module into clusters, referred to as reconstruction input objects. The clustering algorithms for the pixel and SCT detectors are similar in principle, but differ significantly in complexity. Since it operates in only one dimension, the SCT clustering algorithm complexity is linear with respect to the number of hits per module. It consists of a single loop over the SCT strips, accumulating those which are adjacent and active into clusters. The pixel clustering algorithm operates in two dimensions, and so with quadratic complexity, consisting of a double loop over each module, generating clusters for each hit and merging clusters with shared hits where necessary.

Finally, the pixel and SCT clusters are converted to space-points by means of a simple geometric transformation. The pixel clusters are rotated according to the module orientation and then offset by the module position in the global ATLAS coordinate system. The cluster positions in the ATLAS coordinate system are obtained using the module geometry determined by the offline alignment procedure [44]. In the SCT case, one of the modules in each backto-back module pair is rotated at a $40 \mathrm{mrad}$ stereo angle to provide longitudinal information along the module. Clusters from each side of the module are combined into pairs and two-dimensional local positions are derived from these combinations, after which the rotation and translation procedure is identical to that for the pixels. During the decoding the data are checked for errors to ensure increased robustness against detector failures, and the decoded data from each module are cached to remove the need to decode again, should they be requested multiple times.

The TRT data preparation is performed only for the precision tracking in the RoI for which the TRT track extension is included. As in the pixel and SCT case, the data preparation consists of the TRT byte stream decoding and creation of RDOs with the drift time information. The drift time mea- 


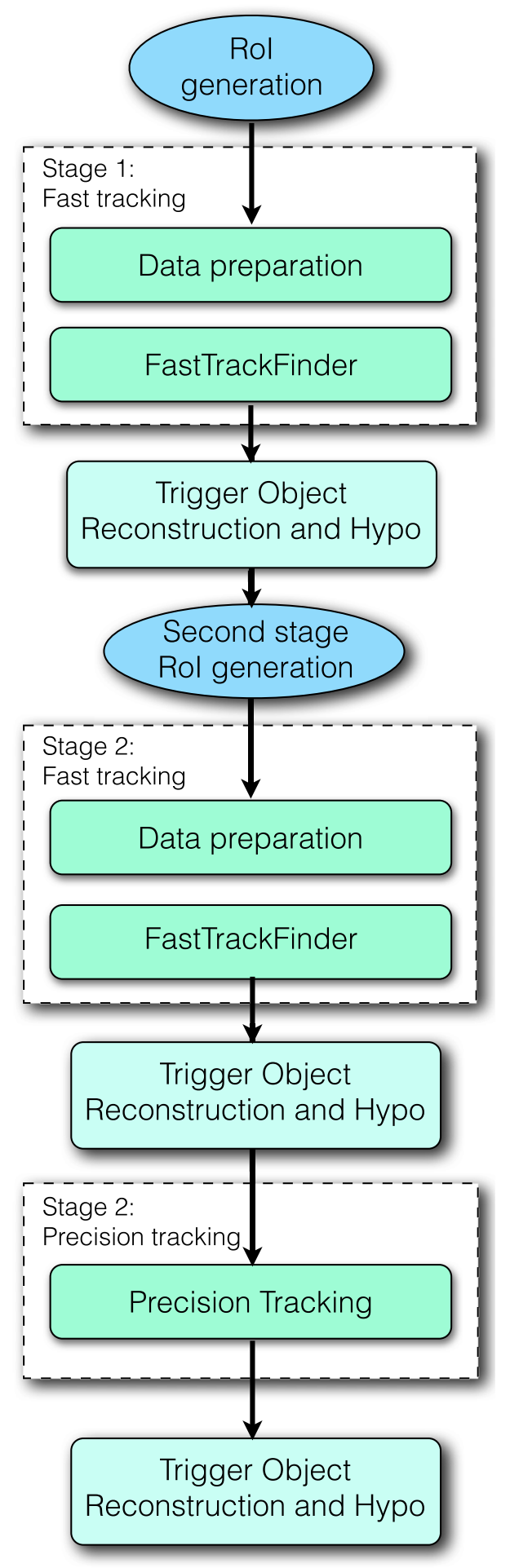

Fig. 7 Schematic illustrating the multistage tracking. Here multistage refers to the multiple passes of the track processing over specific parts of the detector, perhaps with an updated, or otherwise modified RoI. As in the single-stage case, selection can be applied in hypothesis algorithms (hypos) between any of the tracking steps in the two stages

surements are then converted to the drift distances which are stored in the output TRT reconstruction objects.

\subsubsection{Fast tracking}

For Run 2, the fast track finder (FTF) [16] was developed to provide track candidates for use early in the trigger. These track candidates are then used to seed the precision tracking stage. Since the precision tracking provides the track candidates used for the final objects selection in the trigger, the FTF design prioritises track finding efficiency over purity. During the FTF pattern recognition, a search for triplets of space-points (track seeds) is performed in bins of $r$ and approximately 50 sectors in $\phi$, where triplets may also be formed using hits in adjacent sectors at more positive, or more negative, $\phi$, as illustrated schematically in Fig. 6 . The innermost hits from the initial triplets must originate in the pixel detector. Triplet formation starts from a middle space-point and selects outer and inner space-points at larger and smaller radii, respectively. Also illustrated in Fig. 6, the inner and outer pairs of space-points must be compatible with the nominal interaction region along the beam line. In the case of tracking performed within an RoI, this region can be replaced by a restricted $z$-region of the RoI along the beam line if some information about the $z$ position of the interaction is available - the RoI itself is used to pass external information about the expected track $z$ interval to the track seeding algorithm. The triplet track parameters $\phi_{0}$, transverse momentum $p_{\mathrm{T}}$, and transverse impact parameter at the point of closest approach to the beam line $d_{0}$, are estimated using a conformal transformation [45], with the transformation centre placed at the middle space-point and applying cuts on $d_{0}$ and $p_{\mathrm{T}}$. The track transverse momentum parameter from the track reconstruction is signed by the track charge. In practice, $z$ information for the refinement of the RoI at the beam line is only available with high enough resolution after some version of the tracking itself has run.

Initial track candidates are then formed from the track seeds using a simple track finding algorithm which extends the track candidates into further layers to find additional hits, using the offline track-following algorithm [46], but with a modified configuration for faster execution. The trackfollowing algorithm extends track candidates into adjacent layers to identify additional hits, refitting the track candidates as new hits are added. An algorithm to remove duplicate tracks which share track seeds is applied, retaining those tracks of higher quality. These preliminary tracks are then passed to a fast Kalman filter track fitter [47]. To reduce the processing time, hits from the TRT are not used in the FTF.

During the initial track finding, track candidates that have too large an absolute value of $d_{0}$ are rejected in order to keep the contribution from fake tracks to a manageable level. For the muon signature, where the muon candidates are used to seed the $B$-physics signature which reconstructs hadronic resonances with a displaced secondary vertex, the maximum 
allowed $\left|d_{0}\right|$ is $10 \mathrm{~mm}$. For all other signatures, a maximum of $4 \mathrm{~mm}$ is used.

For some signatures, discussed later, a two-stage tracking strategy is used, running additional tracking stages in an updated RoI. In such a case, where the $z$ range of the RoI along the beam line is restricted, the FTF triplet seed formation employs an additional selection where the individual space-point doublets used to construct the triplets are rejected if they do not point back approximately to the restricted region in $z$.

\subsubsection{Precision tracking}

The precision tracking stage takes the FTF tracks as input, and applies a version of the offline tracking algorithms $[37,48]$ configured to run online in the trigger $[49,50]$. This includes a sophisticated algorithm for rejection of duplicate track candidates and clusters previously assigned to tracks but which lie too far from the track trajectory. In addition, the track candidates are extended into the TRT in an attempt to select TRT hits at larger radii to improve the track momentum resolution. The final ID track fit is then performed using a more precise global $\chi^{2}$ fitter algorithm [51] from code used for offline reconstruction. These stages are combined as aspects of the offline ambiguity solver algorithm discussed in Sect. 3.1.

Since the rate of event processing for the precision tracking is generally significantly lower than for the fast tracking, more detailed handling of the detector conditions is possible. This includes better compensation for detector effects such as inactive sensors or calibration corrections. The resulting precision tracks are therefore much closer in performance to the offline tracks than the fast tracks. By necessity, the precision tracking must generally make use of the detector conditions as they are known during the initial data taking for the run, and, unlike the offline tracking, cannot benefit from the improved knowledge of those conditions obtained during the subsequent offline processing. One exception where information must be updated during the data taking concerns knowledge of the beam line position. As the subsequent offline beamspot information is not available, a custom beam line vertex trigger is used, which runs the fast tracking, and then uses these tracks to run a fast vertex algorithm to establish the interaction region position in the transverse plane as a function of the $z$ position itself. This is then used to update the beam line position used online whenever this position has moved by a predetermined amount $[3,27]$.

Since the precision tracking uses the tracks and clusters identified by the fast tracking, the precision tracking efficiency, by construction, cannot exceed that of the fast tracking. Overall, the primary purpose of the precision tracking is to perform a higher quality fit to improve the purity and qual- ity of the trigger tracks relative to those tracks reconstructed offline.

\subsubsection{Vertex reconstruction}

During Run 2, two vertex algorithms were used online: a histogramming based algorithm, and the offline iterative vertex algorithm [52,53]. Typically, trigger signatures use only the offline vertex algorithm. For the case of the $b$-jet trigger [10] however, both vertex algorithms were employed to maximise the vertex finding efficiency. The simple histogramming algorithm functions by first histogramming the $z_{0}$ position for the point of closest approach to the beam line of each track. It then calculates the vertex $z$ position using the mean of the bin centres weighted by the number of tracks in each bin for the group of adjacent bins within the $1 \mathrm{~mm}$ sliding window which contain the largest number of tracks. All tracks passing some basic quality selection are used and are weighted equally. The second algorithm, based on the offline vertex finder, with some minor modifications to run online, iteratively clusters tracks to determine the positions of vertex candidates, sorting the resulting vertex candidates by the sum of the squared transverse momenta of the tracks assigned to each vertex.

In both cases, the vertex algorithm runs only on tracks that have been reconstructed in the relevant RoI of the track finding. For the leptonic triggers the offline based algorithm is usually executed using the precision tracks from the final stage of processing. For the $b$-jet trigger, both vertex algorithms are executed using tracks from a specific additional vertex tracking stage before the dedicated tracking in the $b$-jet RoI.

\subsection{Multistage tracking}

Although the fast and precision tracking run in distinct stages or steps - often separated by additional non-tracking algorithms and event rejection - running both algorithms sequentially in a single RoI is in general considered to be processing in a single tracking stage. This is because there is only a single pass through the full set of tracking algorithms over any given RoI. Where multiple passes of aspects of the tracking are intentionally performed over similar, but not identical, regions of the detector, and where the second pass is in a different RoI constructed to overlap with, extend, or update the RoI of the first pass, this is referred to as multistage tracking. In this case, each set of steps within a specific RoI constitutes a single tracking stage. Such a case might be where the first stage runs the fast tracking in a narrow RoI, and a subsequent stage again runs the fast tracking in a new RoI, along the same direction, but wider. This is illustrated for a generic multistage signature in Fig. 7. 


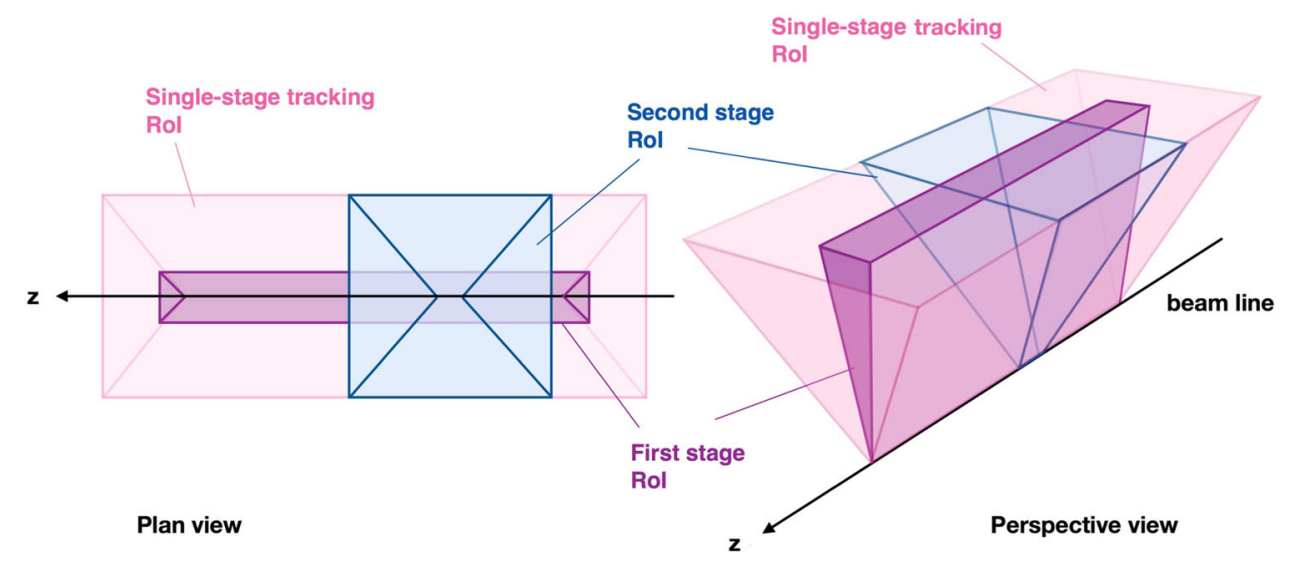

Fig. 8 Schematic illustrating the RoI from the single-stage and twostage tau lepton trigger tracking, shown in plan view ( $x-z$ plane) along the transverse direction and in perspective view. The $z$-axis is along the beam line. The combined tracking volume of the 1st and 2nd stage RoI in the two-stage tracking approach is significantly smaller than the RoI in the single-stage tracking scheme
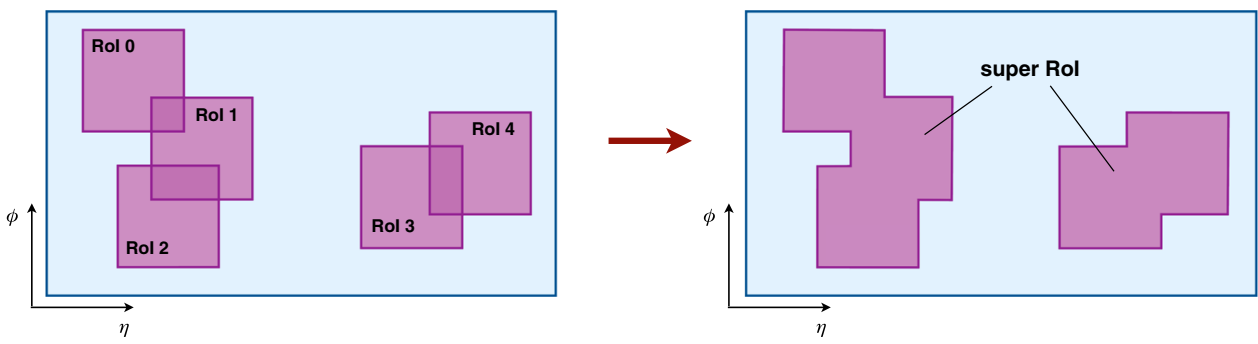

Fig. 9 Schematic illustrating how the super RoI for the vertex tracking is created from all the trigger jets reconstructed with $E_{\mathrm{T}}>30 \mathrm{GeV}$. The RoI around each initial jet direction is fully extended in $z$ along the beam line

For the hadronic tau trigger, it is useful to run the tracking in a larger RoI than that used, for instance, in the electron trigger, to allow for the opening angle of the tracks from three-prong tau decays $[8,13]$. For Run 1 , this was done by using a single RoI, wider in $\eta-\phi$ but fully extended along the beam line, which consequently was one of the most timeconsuming aspects of the tracking in Run 1 . To limit the tracking CPU usage in the wider RoI, a two-stage processing approach was implemented for the tau trigger in Run 2. In the first stage, the position of the tau event vertex along the beam line is identified by executing the fast tracking in a narrow RoI with a full width of 0.2 in both $\eta$ and $\phi$, directed towards the tau candidate calorimeter cluster, but fully extended along the beam line in the range $|z|<225 \mathrm{~mm}$ to identify the leading $p_{\mathrm{T}}$ tau decay tracks. The second stage executes the fast tracking again, followed by the precision tracking for the tracks found in this second fast tracking stage, but this time in a wider RoI with a full width 0.8 in both $\eta$ and $\phi$, centred on the $z$ position of the leading track identified by the first stage and limited to $|\Delta z|<10 \mathrm{~mm}$ relative to this leading track. The RoI from these different single-stage and two-stage strategies are illustrated schematically in Fig. 8.

For comparison purposes, during the commissioning for the Run 2 data taking, the tau lepton triggers were also executed in a single-stage mode, similar to that used for Run 1
[54], running the fast track finder only once in a wide RoI in $\eta$ and $\phi$ and fully extended along the beam line, followed by the precision tracking again in this same RoI. As a result of the time saved in the processing of the second stage because of the narrow $z$ extent along the beam line, the actual $\eta-\phi$ width of the second stage RoI can be made larger than would otherwise be possible running only in a single stage with the full $z$ extent.

In Run 1 , the $b$-jet trigger ran the full tracking individually in the full $z$-width RoI centred on jets from the trigger [9] with a wide $\eta-\phi$ extent. However, for Run 2, the increased beam energy and interaction multiplicity meant that this approach was inappropriate, resulting in too many RoI with too large an overlap such that the degree of processing of overlapping parts of the detector became prohibitive.

Consequently, for the Run $2 b$-jet trigger [10] a new multistage tracking strategy was adopted with a new initial tracking stage, running tracking specifically to identify the likely event vertex $z$ position for use in the second stage. For the second stage, separate RoI about each jet axis are used as in Run 1, but with each RoI specified more tightly at the beam line about the $z$ vertex position identified in the first stage. For the tracking in the initial vertex stage, all jets identified by the jet trigger with transverse energy $E_{\mathrm{T}}>30 \mathrm{GeV}$ are considered, and tracks are reconstructed 
with the fast tracking algorithm in a narrow region with full widths of 0.2 in $\eta$ and $\phi$ around the jet axis for each jet, but with $|z|<225 \mathrm{~mm}$ along the beam line. ${ }^{3}$ In order to prevent the multiple processing of regions of the detector that overlap, before running the fast tracking, the RoI about each jet axis are first aggregated into a single super RoI for the event. This super RoI is then used by the data preparation of the first stage vertex tracking to determine which detector elements - in this case individual silicon modules from the pixel and SCT detectors - should be read out for the subsequent processing. This happens only once per event for the single super RoI, which can include non-overlapping regions. This is illustrated in Fig. 9, which shows the aggregation of the individual narrow jet RoI into a single, more complicated region used by the data preparation. With only the data from these detector elements corresponding to the RoI about the jet directions, the tracking then runs as if it were processing the entire detector.

Following this stage, the tracks identified in the super RoI are used for the primary vertex reconstruction [55]. This vertex is used to define wider RoI about each jet axis, with $|\Delta \eta|<0.4$ and $|\Delta \phi|<0.4$ with respect to the jet axis, but with $|\Delta z|<10 \mathrm{~mm}$ relative to the primary vertex $z$ position. These RoI are then used for the second-stage reconstruction which again runs the fast tracking but this time in the wider $\eta$ and $\phi$ regions about the jets. This is then followed by the precision tracking, secondary vertexing and $b$-tagging algorithms. For the vertex stage, different vertex algorithms are available. As in the case of the tau multistage tracking, the time saved by running the jet tracking in very narrow RoI in $z$ about the vertex position allows the jet tracking stage to execute in a wider RoI than would be possible with a single-stage processing.

In Run 2 this same approach was also adopted for some of the standard jet triggers in order to be able to run the global sequential calibration [56]. This makes use of the information from the precision track reconstruction in the jets to improve the jet energy resolution measured with respect to the offline jets. This allows a tighter selection on the jet transverse momentum [57,58], closer to that used offline, thus reducing the rate from the jet trigger.

\section{Inner detector trigger timing}

In this section, timing measurements for the tracking related algorithms used in the inner detector trigger in Run 2 are presented. Timing measurements for the different tracking related algorithms were taken online, using the trigger cost monitoring framework [27]. Unless otherwise stated, the timing measurements were recorded during a typical physics

${ }^{3}$ In 2016 the constituent RoI full width in $\eta$ and $\phi$ was 0.4 .

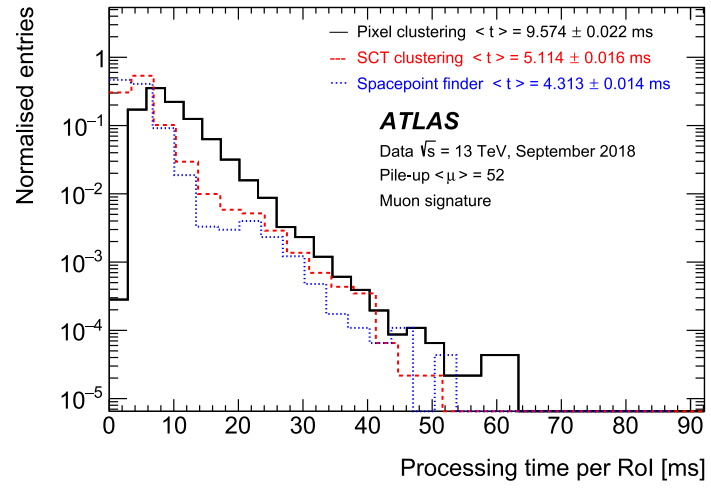

(a)

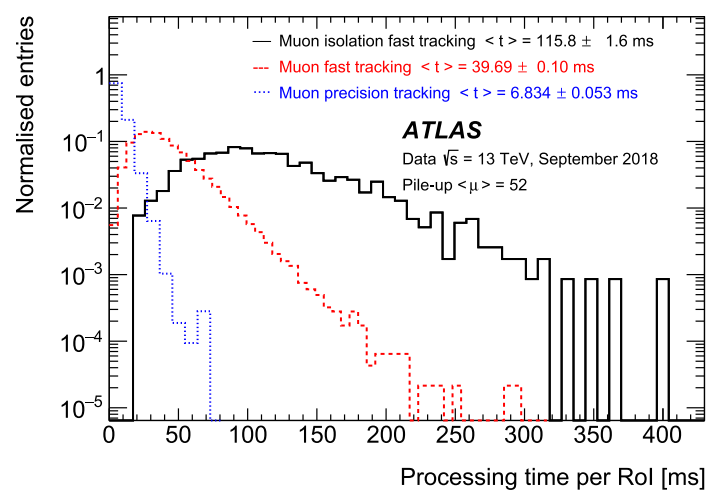

(b)

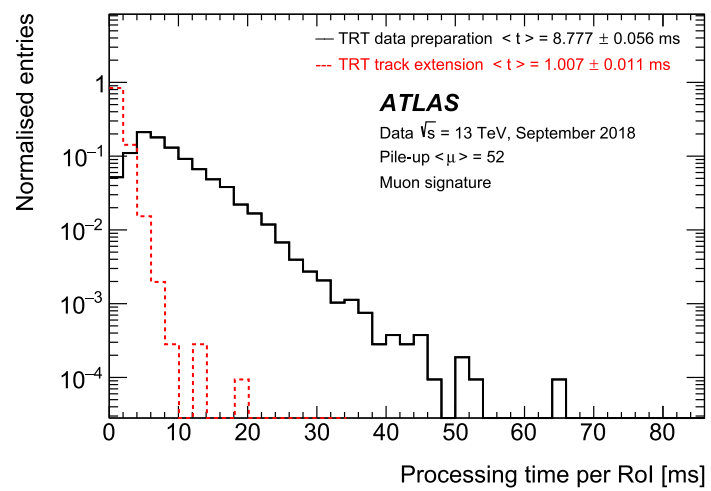

(c)

Fig. 10 The processing time for muon tracking related algorithms; a the data preparation times, $\mathbf{b}$ the tracking algorithm times, and $\mathbf{c}$ the TRT data processing

run, on Saturday, September 29th, 2018 with a mean pileup interaction multiplicity of 52 at the start, and 19 at the end of the run. The full timing distributions are presented for the start of the run only.

The retrieval and data preparation for the silicon detectors prior to running the tracking algorithms themselves is typically reasonably fast, but is still a significant contribution to the overall processing time. The subsequent fast track reconstruction then precedes the execution of hypothesis algorithms which combine the data from the calorimeters or muon 


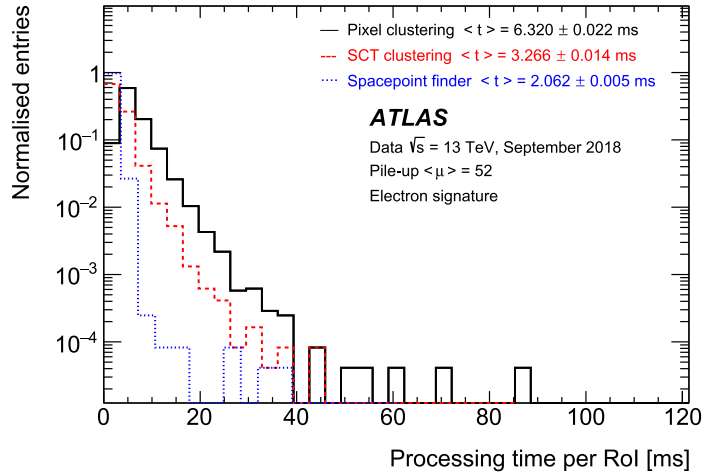

(a)

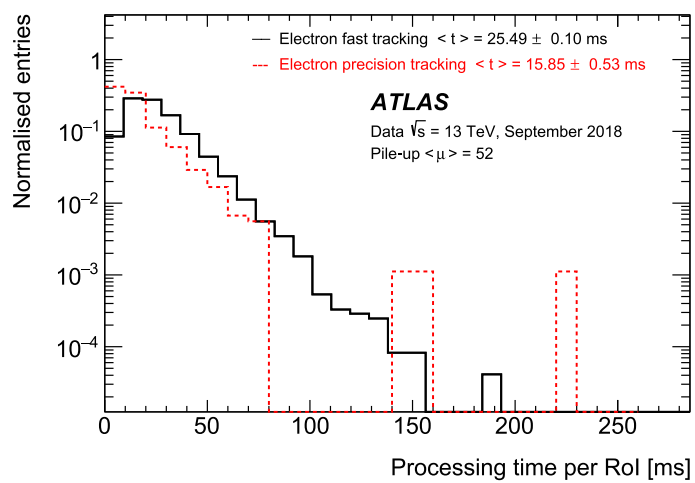

(b)

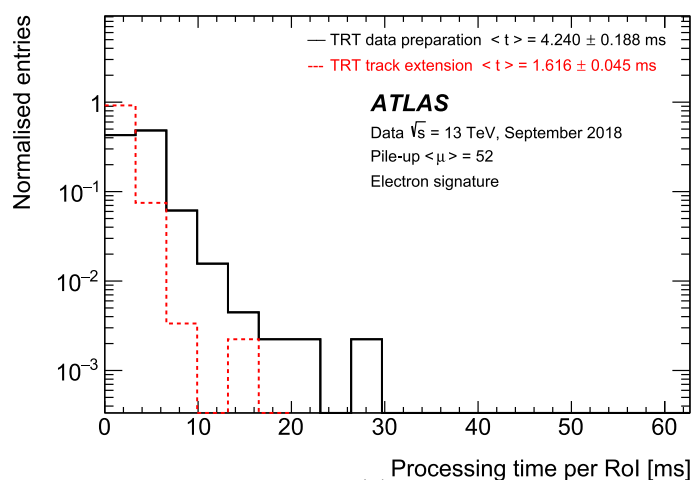

(c)

Fig. 11 The processing time for electron tracking related algorithms; $\mathbf{a}$ the data preparation times, $\mathbf{b}$ the tracking algorithm times, and $\mathbf{c}$ the TRT data processing

spectrometer with the ID tracks to further reduce the rate. Following this reduction, the precision tracking stage is executed, and the precision tracks can be extended into the TRT, which requires the TRT data preparation and track extension algorithms. Subsequent algorithms may use the tracks in combination with clusters reconstructed in the calorimeter, or perform a combined fit using the information from the muon spectrometer and the inner detector. A discussion of some of these other algorithms can be found elsewhere $[59,60]$.

A vertex finding algorithm can also be executed using the tracks from any stage in the processing, typically following the precision tracking, but also using the tracks from the fast track finder, for example, following the $b$-jet vertex tracking. In the case of the multistage tracking, the data preparation may be executed a second time in the wider regions of the detector. However, the times for the data preparation are not recorded separately for the different stages, and so for this analysis are aggregated into a single distribution for each specific trigger.

\subsection{Algorithm execution time in the muon trigger}

The principal processing times for the algorithms in the muon signature are shown in Fig. 10 for the standard full RoI width of 0.2 in both $\eta$ and $\phi$. Some triggers run a second isolation tracking stage in a wider $\eta-\phi$ region, after the first round of precision tracking, to determine whether tracks are isolated with respect to other tracks from the interaction. In this case the data preparation and fast track finder are executed a second time but in a wider RoI in $\eta$ and $\phi$, but still extended by the full length in $z$ along the beam line. The precision tracking then runs a second time on the tracks in this larger RoI. As in the case of the data preparation, information to distinguish between the first and second execution of the precision tracking within any specific muon trigger is not available so only the precision tracking time of the first pass is shown here.

The space-point preparation is generally very fast, as can be seen in Fig. 10a, each algorithm having a mean processing time per RoI of less than $10 \mathrm{~ms}$.

Figure 10b illustrates that the fast tracking in the first stage processing is reasonably fast with a mean execution time of $40 \mathrm{~ms}$ and a maximum at approximately $300 \mathrm{~ms}$. The precision tracking has a mean execution time of less than $7 \mathrm{~ms}$.

The second stage muon isolation fast track processing with the fully extended $z$ range, and wider $\eta-\phi$ size is significantly slower, with a mean processing time of approximately $116 \mathrm{~ms}$. This is only executed on RoI where a trigger muon candidate has already been confirmed such that the rate should be significantly lower than for the first stage RoI processing. The TRT extension is only performed for the precision tracks and the execution time from Fig. 10c is under $10 \mathrm{~ms}$.

One additional factor in the processing of the muon trigger is that prior to the execution of the first stage processing with the fast tracking, the RoI is determined by the muon spectrometer trigger [6], which calculates an updated RoI position based on the first processing of the spectrometer data in the HLT. Partly because of the toroidal field in the muon spectrometer, the resolution of the tracks in $z$ extrapolated back to the beam line for the spectrometer-only muon candidates is not good enough to refine the $z$ position of the RoI, so the full extent in $z$ along the beam line is required. Additionally, in the transition regions between the barrel and endcap detectors, with pseudorapidities near \pm 1 , the $\eta$ reso- 


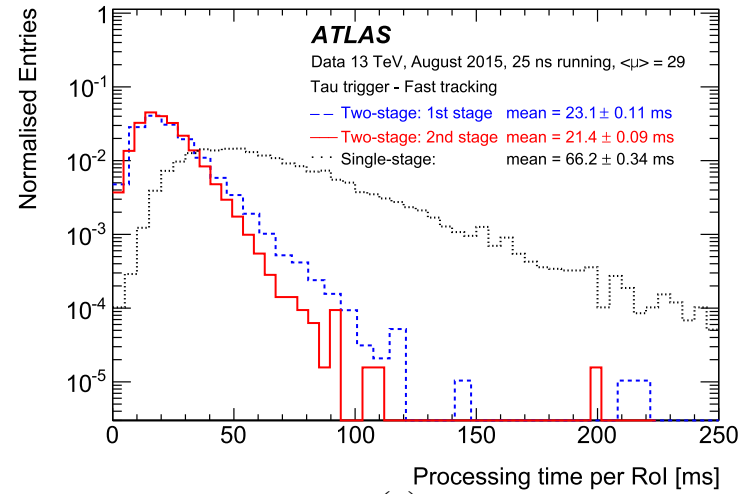

(a)

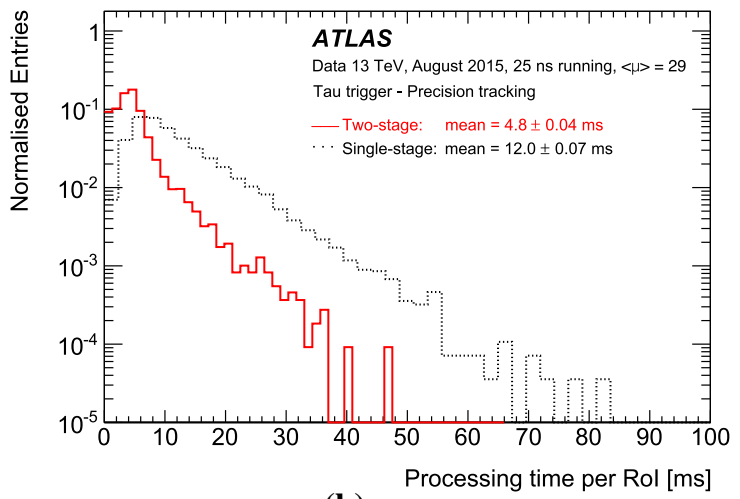

(b)

Fig. 12 The ID trigger tau tracking processing time for a the fast track finder and $\mathbf{b}$ the precision tracking comparing the single-stage and two-stage tracking approach

lution of the spectrometer-only candidates is poor, and so the RoI for the ID tracking can be made commensurately wider to ensure that the space-points for the ID track are contained within the RoI.

\subsection{Algorithm execution time in the electron trigger}

The processing for the electron triggers is slightly less complex than for the muons, since it does not run a second stage. As such both the fast and precision tracking run in the same RoI, produced after the first calorimeter reconstruction algorithm. The RoI dimensions are the same as for the first stage from the muon signature. Between the fast tracking and precision tracking, additional reconstruction algorithms combining the calorimeter and tracking information to produce electron candidates can be executed. Hypothesis algorithms are then executed to select on these electron candidates to reduce the rate before the precision tracking. The principal algorithm processing times for the different steps in the sequence can be seen in Fig. 11.

Here the data preparation stages are very quick - typically less than $7 \mathrm{~ms}$. The mean processing time in data from late 2018 was approximately $26 \mathrm{~ms}$ for the fast tracking and under $16 \mathrm{~ms}$ for the precision tracking. The full TRT extension following the precision tracking took under $6 \mathrm{~ms}$.

\subsection{Algorithm execution time in the tau trigger}

As an example of the benefits afforded by using the multistage approach for the tau processing discussed in Sect. 3.4, the time taken by the tau tracking in both the singlestage and two-stage variants is shown in Fig. 12. This is from an ATLAS data-taking run with $25 \mathrm{~ns}$ bunch spacing taken during the detector commissioning from 2015 where the mean pile-up interaction multiplicity was 29 . For subsequent production luminosity running in 2016 and onwards, only the two-stage version was running for all tau triggers.
Figure 12a shows the processing times per RoI for the fast tracking stages: individually for the first and second stages of the two-stage tracking, and for the single-stage tracking with the original wider $\operatorname{RoI}$ in $\eta, \phi$ and $z$. The fast tracking in the single-stage has a mean execution time of approximately $66 \mathrm{~ms}$, with a very long tail. In contrast, the first stage tracking with an RoI that is wide only in the $z$ direction has a mean execution time of $23 \mathrm{~ms}$, driven predominantly by the narrower RoI width in $\phi$. The second stage tracking, although wider in $\eta$ and $\phi$ even than the RoI used in the single-stage, takes only $21 \mathrm{~ms}$ on average because of the significant reduction in the RoI $z$-width along the beam line. Figure $12 \mathrm{~b}$ shows a comparison of the processing time per RoI for the precision tracking. The precision tracking in the two-stage strategy executes faster, with a mean of $4.8 \mathrm{~ms}$ compared to $12 \mathrm{~ms}$ for the single-stage tracking.

The execution times for the tracking related algorithms for the tau signature from production luminosity running late in 2018 are shown in Fig. 13. In this case the pile-up interaction multiplicity, with a peak mean value of approximately 52 interactions per bunch crossing, is significantly larger than for the 2015 data-taking from the previous figure. In addition, at the start of running in 2017, the $z$-width of the second stage tau RoI was reduced further. Here the first stage fast track finder execution time in the full $z$-width RoI is $35 \mathrm{~ms}$ and that of the second stage is $23 \mathrm{~ms}$. The precision tracking takes $10 \mathrm{~ms}$.

A significant time - approximately $13 \mathrm{~ms}$ - is taken by the calculation of the TRT drift circles for the extension of the precision tracks into the TRT.

\subsection{Algorithm execution time in the $b$-jet trigger and vertexing}

The $b$-jet trigger runs as a multistage trigger with regard to the tracking. Before the $b$-jet specific parts of the trigger are 


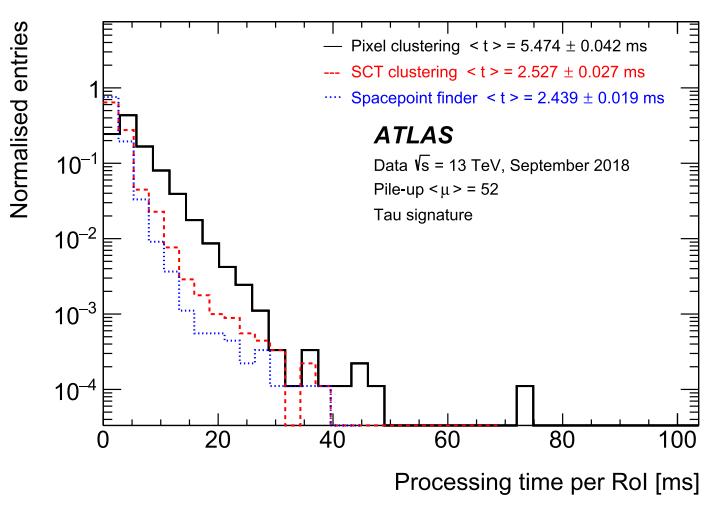

(a)

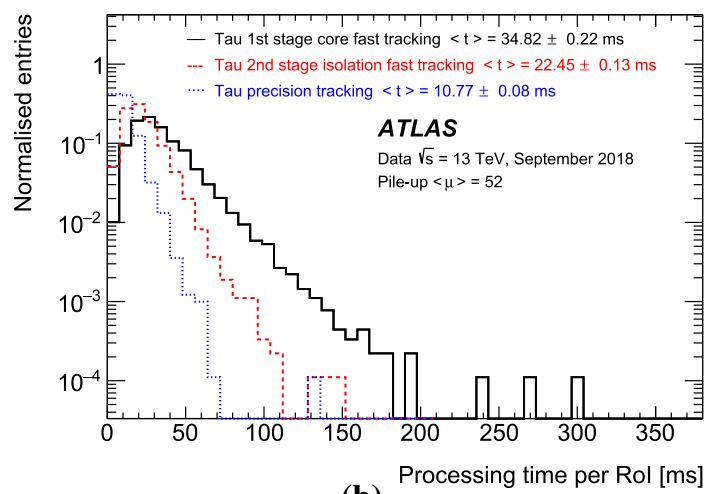

(b)

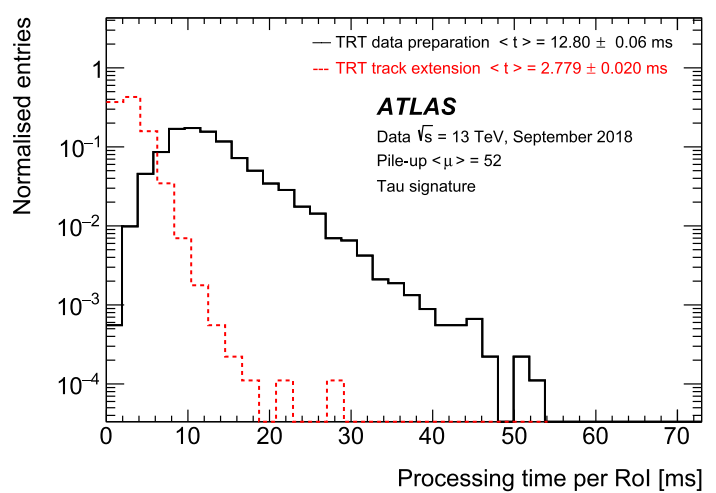

(c)

Fig. 13 The processing time for tau tracking related algorithms; a the data preparation times, $\mathbf{b}$ the tracking algorithm times, and $\mathbf{c}$ the TRT data processing

executed, the trigger runs as a standard jet trigger, running the jet finding in the full detector [9].

Following this, the first stage of the $b$-jet trigger takes the collection of jets from the full detector, and creates a separate RoI about each jet with $E_{\mathrm{T}}>30 \mathrm{GeV}$. These are aggregated into a super RoI as discussed in Sect. 3.4. The data preparation and fast tracking for the vertexing runs in this super RoI, followed by a vertex algorithm. The vertex position is then used to refine the $z$ position of each jet RoI, which are also widened in $\eta$ and $\phi$.

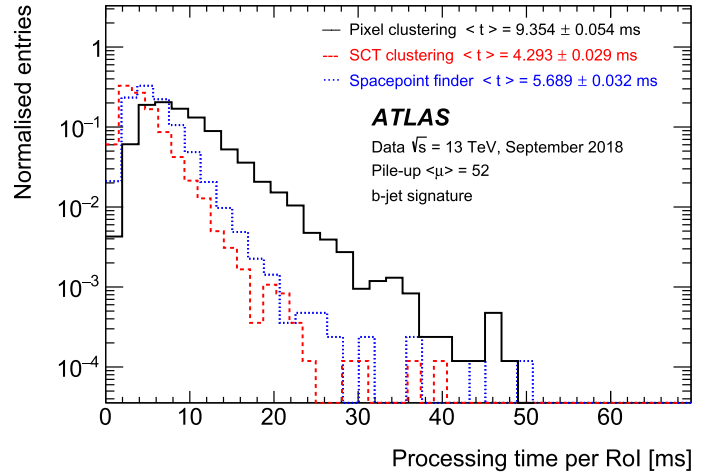

(a)

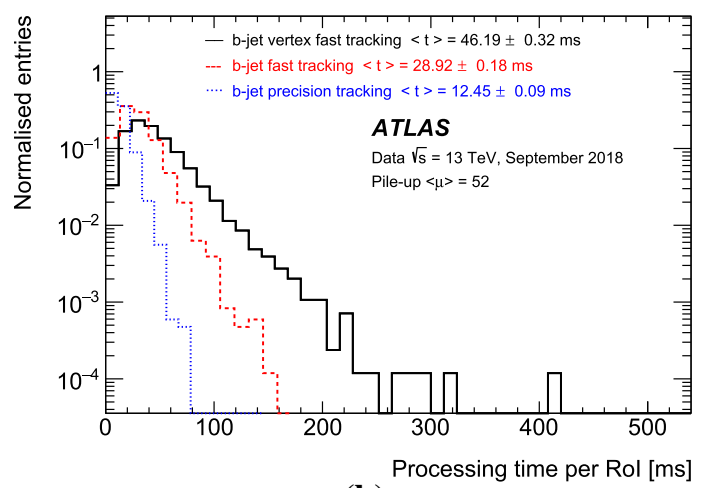

(b)

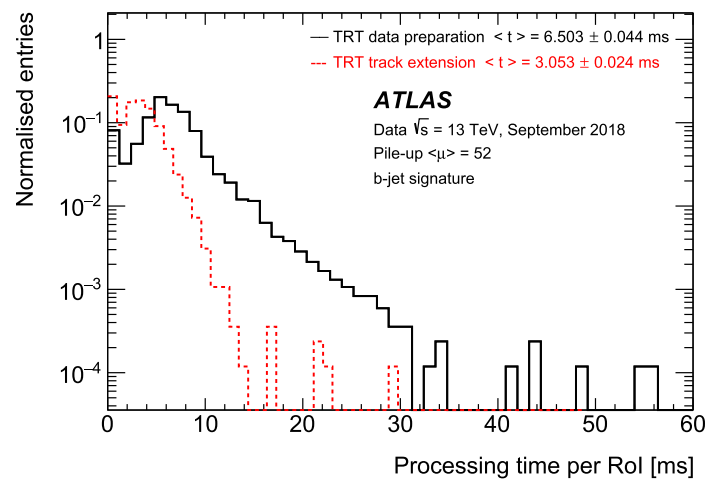

(c)

Fig. 14 The processing time for $b$-jet tracking related algorithms; a the data preparation times, $\mathbf{b}$ the tracking algorithm times, and $\mathbf{c}$ the TRT data processing

Subsequently, the data preparation, fast tracking and precision tracking are executed in each jet RoI individually. The precision tracks are then used for the subsequent $b$ tagging algorithms. For the standard jet triggers with the global sequential calibration, the tracks are used for the additional correction of the jet $E_{\mathrm{T}}$ before the $b$-tagging is performed.

The distribution of processing time for the ID data preparation can be seen in Fig. 14a, which includes the data preparation for both the vertex tracking stage and the second, RoI 
based, $b$-jet tracking stage. The mean execution time for each algorithm is under $10 \mathrm{~ms}$.

Due to the larger volume of the detector to be processed, the vertex tracking for the fast tracking is the longest of the tracking stages, with a mean execution time of $46 \mathrm{~ms}$, shown in Fig. 14b. However, caching of the objects reconstructed by the trigger means that the vertex tracking need be executed only once per event, with the output being shared between all jet triggers. The subsequent tracking within the individual jet RoI has a mean execution time of $29 \mathrm{~ms}$ and $12 \mathrm{~ms}$ per jet RoI for the fast and precision tracking algorithms respectively. The TRT extension for the precision tracks takes a total of approximately $10 \mathrm{~ms}$ per jet RoI, as illustrated in Fig. 14c.

Compared to the tracking itself, the execution time for the vertexing is rather fast. Shown in Fig. 15c, the execution time for the offline based vertex algorithm is a little over $1 \mathrm{~ms}$, whereas the histogram based vertex algorithm takes less than $0.5 \mathrm{~ms}$. Also shown in Fig. 15 are the times for the execution of the vertex algorithm in the muon, electron and tau signatures. In each case of the single-RoI leptonic triggers the offline based algorithm takes less than $1 \mathrm{~ms}$.

\subsection{Total tracking time per event}

The total time spent by the tracking related algorithms in the event processing from this same typical run from September 2018 can be seen in Fig. 16.

This figure shows the average of the total execution time per event for each algorithm of the ID trigger, and how this varies as the mean pile-up interaction multiplicity changes throughout the run. The total time for each algorithm is dependent on the details of the trigger menu, which determines how often and for which trigger thresholds the tracking will be executed.

The execution times for the data preparation in the silicon detectors are clearly seen to decrease for falling pileupmultiplicities. As expected from the execution time for each algorithm for the different signatures, the data preparation for the pixel detector is the slowest, taking $27 \mathrm{~ms}$ overall in the trigger per event for the high interaction multiplicities, with $\langle\mu\rangle=52$, at the start of the fill.

The fast tracking is clearly the longest with a mean of approximately $155 \mathrm{~ms}$ per event in total at high interaction multiplicities, and has a strong non-linear behaviour with increasing multiplicities. The precision tracking in contrast has a more linear dependence, and takes only a third as long as the fast tracking in total. This is due to the time per call being lower, and the precision tracking being executed less often due to the early rejection in the trigger, which terminates processing before running the precision tracking algorithm.

The TRT data preparation and track extension are both reasonably fast and exhibit a more linear behaviour, since they are only executed on tracks from the precision tracking.
Finally, the vertex algorithms are seen to be very fast overall. The histogramming algorithm is in principle only executed once per event and, as in the previous section, is seen to have a mean time per call at high pile-up of around 0.3 ms, exhibiting only a slight dependence on the pile-up interaction multiplicity. In contrast, the offline vertex algorithm also runs once per event for the $b$-jet vertexing, but in addition is executed once per RoI for the other signatures. The execution time shows a small dependence on the interaction multiplicity, and consists mostly of the time taken by the single execution in the $b$-jet vertex tracking.

Overall, the total time spent in all the tracking related algorithms discussed here is approximately $290 \mathrm{~ms}$ per event at high pile-up multiplicities, decreasing to a little more than $90 \mathrm{~ms}$ for the lower multiplicities.

\section{High-level trigger tracking performance}

\subsection{Data selection}

For the performance analyses of the muon and electron signatures, the full available integrated luminosity for the 20162018 running period is used. This is possible since the processing for these signatures did not change significantly during the data taking period.

For the case of the tau and $b$-jet analyses, which ran twostage tracking with a modified second stage RoI, significant changes were made to the reconstruction of either the first, or the second stages over the three years. These included modifications to the second stage seed finding, and so only the results for 2018 are presented in full detail. For each of the three years, 2016, 2017, and 2018 individually, brief comparisons of the efficiencies in each year are presented. For the tau signature, the full available integrated luminosity from each year is used. For the $b$-jet analyses only a subsample from each year, sufficient to provide a small statistical uncertainty is used.

In all cases, only events where the data quality was determined to be good for physics analyses are used [61].

The tracking efficiency for the trigger with respect to the offline tracking is determined using a number of support triggers. These triggers are essentially identical to the physics triggers, and operate by reconstructing the tracks in the trigger as normal, but then selecting on the objects reconstructed in the muon spectrometer or calorimeter only, with no selection on the tracking information from the inner detector. In this way, it is possible to estimate the efficiency of the tracking, unbiased by the ID track reconstruction itself. One caveat is that because the trigger does not select on the inner detector track information, the prescale for the triggers generally needs to be kept high to restrict the rate. Since the trigger objects used for the selection do not include any tracking, 


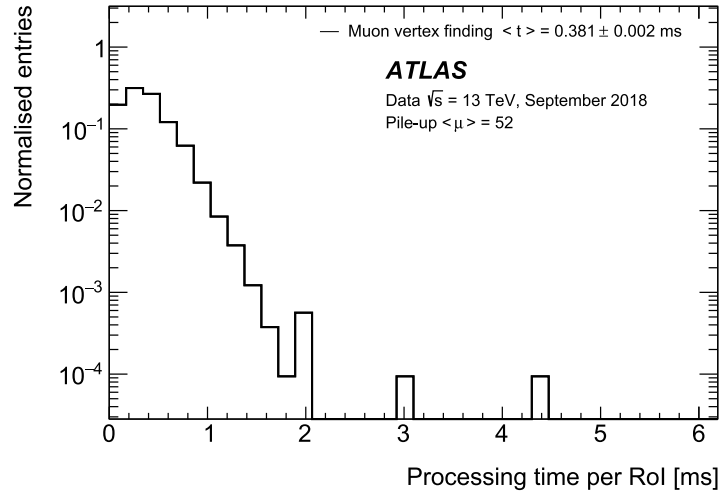

(a)

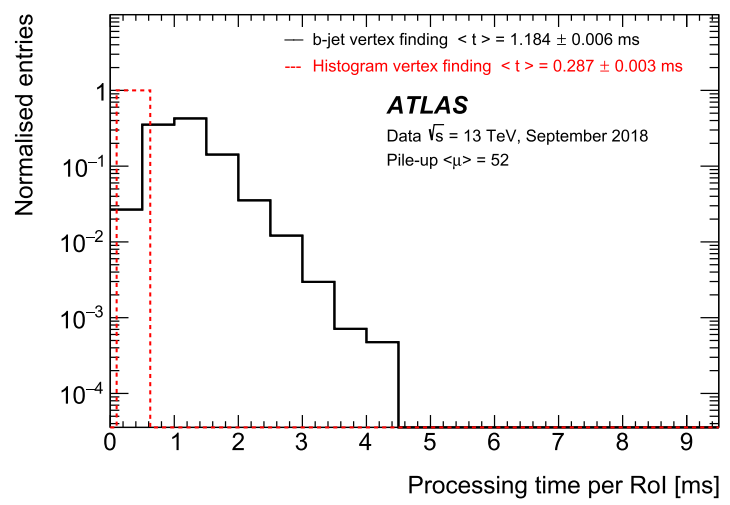

(c)

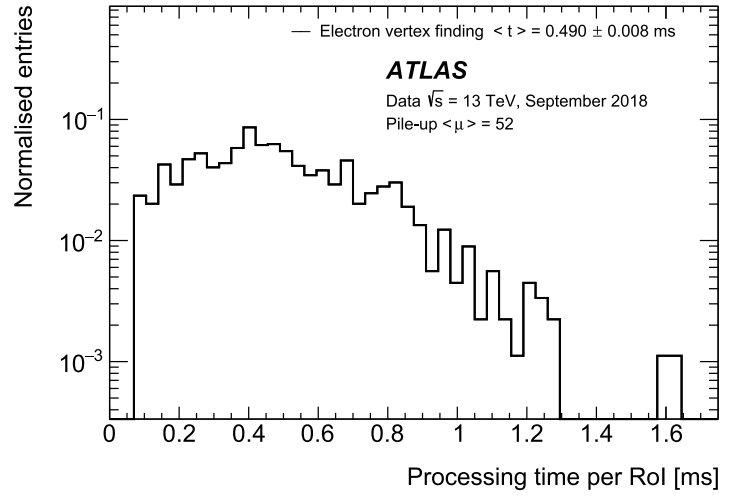

(b)

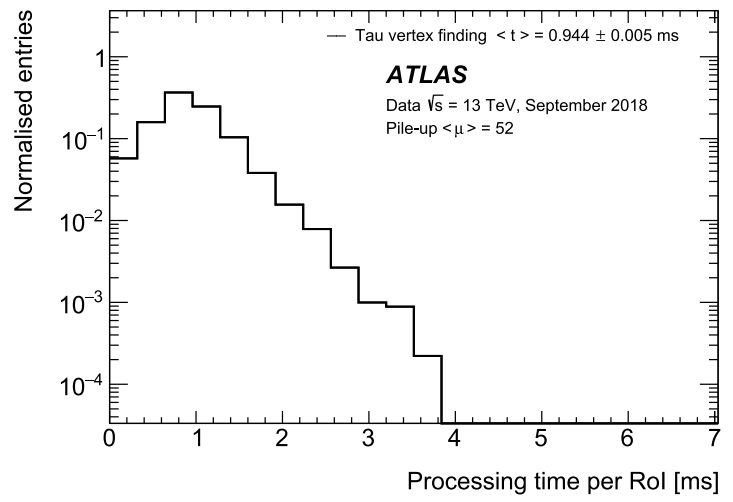

(d)

Fig. 15 The processing times for trigger vertex algorithms; $\mathbf{a}, \mathbf{b}$ for the muon and electron signatures, $\mathbf{c}$ for the $b$-jet vertex tracking, and $\mathbf{d}$ for the tau signature

the fraction of background selected by the trigger will of necessity be significantly higher than the fully selecting triggers, e.g. electron candidates from these triggers will have been selected without any requirement on track multiplicity, instead being based purely on the clusters reconstructed in the calorimeter. As a consequence there may be a large contribution from narrow QCD jets which resemble an electromagnetic cluster. For each of the analyses, any such background is reduced by the selection of a reference sample using the full offline reconstructed objects, against which the performance is measured. Consequently, the sample of good offline objects, including the offline tracks, that are used to monitor the trigger may be statistically limited in regions of phase space.

\subsection{Track selection}

For the measurement of the efficiency and resolution, the ensemble of inner detector trigger tracks within the RoI are first matched to the selected offline reference objects using a modified solution to the stable marriage problem [62] - the closest matches taken only from those tracks which match within a loose preselection cone of size $\Delta R=$
$\sqrt{(\Delta \eta)^{2}+(\Delta \phi)^{2}}=0.05$ around an offline reconstructed track. For any given offline track, the closest matching trigger track is chosen as a match if, and only if, it does not match more closely any other offline track.

Efficiencies are then measured by taking the correlated ratio of the number of offline reference objects that have such a matched trigger track to the total number of offline objects passing the selection.

Since the residual distributions themselves may have very long non-Gaussian tails, the resolution is estimated by taking the root-mean-square of the central $95 \%$ of the residual distribution. This is then scaled by the inverse of the root-mean-square of the central $95 \%$ of a Gaussian distribution with unit standard deviation, such that for any Gaussian residual distribution the true width would be obtained.

Reference objects for the analyses are chosen to satisfy the full offline selection criteria for such objects, similar to those used for physics analyses, but with additional selection. These are discussed in detail later in the text.

In all cases, offline candidate tracks are required to lie within the RoI used for the trigger reconstruction, since tracks outside the RoI would not be reconstructable. An additional 


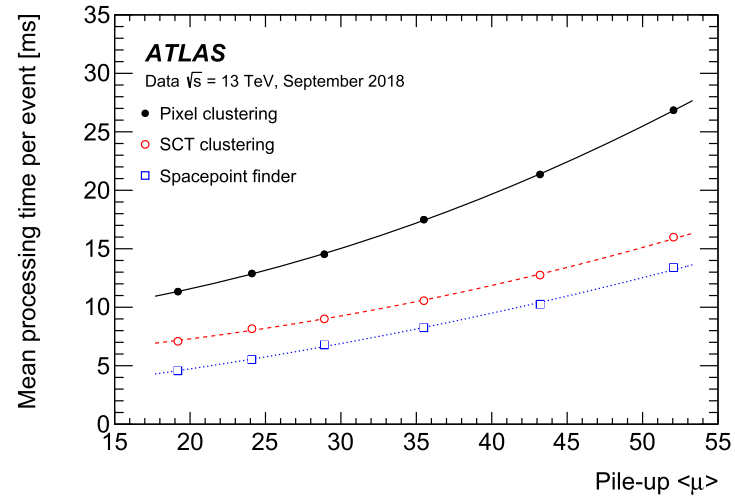

(a)

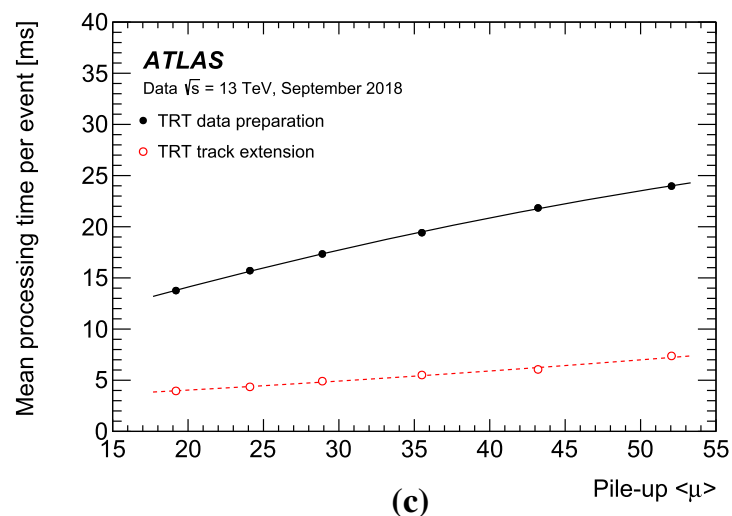

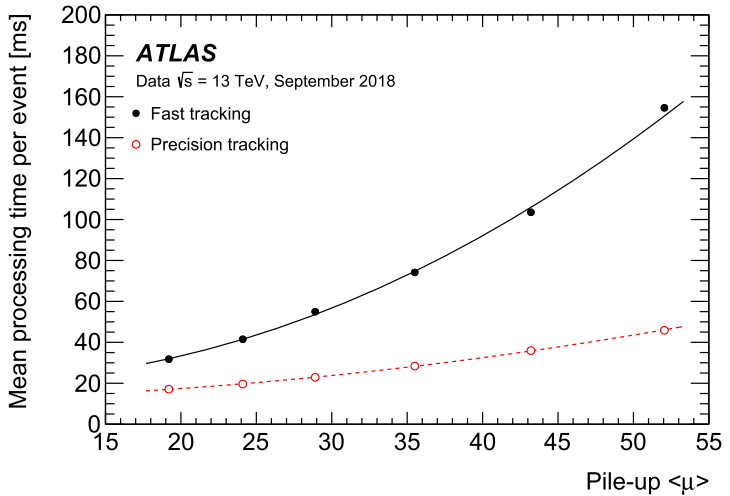

(b)

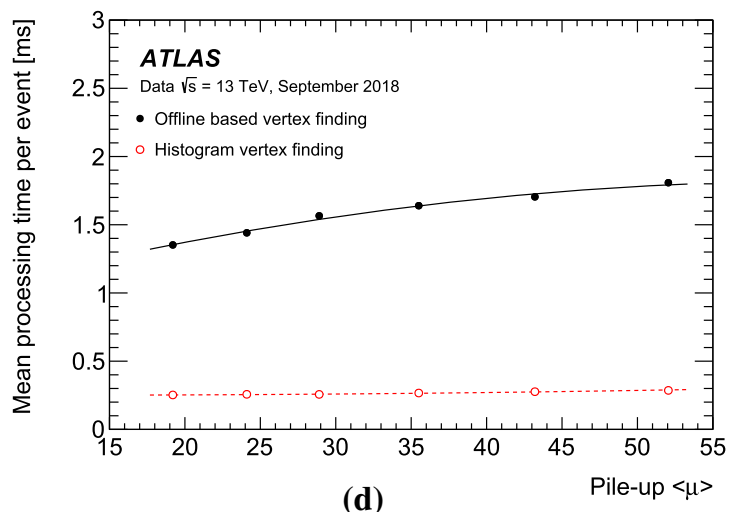

(d)

Fig. 16 The mean of the total processing time per event for the tracking related algorithms; a the data preparation for the silicon detectors, $\mathbf{b}$ the fast, and precision tracking, $\mathbf{c}$ the TRT data preparation and track extension, and $\mathbf{d}$ the vertexing

requirement ensures that offline tracks are used only if the transverse impact parameter for the point of closest approach to the beam line, and the $z$ position relative to any relevant offline vertex, are both less than $10 \mathrm{~mm}$.

For the remaining discussion no distinction is made between positively and negatively charged particles for the transverse momentum and only the absolute value is considered.

\subsection{Tracking in the muon signature}

For the muon signature the HLT uses triggers $[4,25]$ with muon transverse momentum reconstruction down to $4 \mathrm{GeV}$. The performance of the trigger tracking is therefore studied using a range of support triggers covering the region down to this threshold.

Figure 17 shows the tracking efficiency of the ID trigger for medium quality offline muon candidates [63]. The efficiency integrated over $p_{\mathrm{T}}$ is shown for the fast tracking and the precision tracking for two representative thresholds for the offline muon selection: $p_{\mathrm{T}}>4 \mathrm{GeV}$ corresponding to the lowest trigger threshold, and $p_{\mathrm{T}}>20 \mathrm{GeV}$. The efficiency is shown as a function of the offline muon candidate track parameters and the mean number of pile-up interactions per event. The selection of the offline muon candidates is slightly biased by the muon spectrometer trigger selection near the threshold, but this does not affect the measurements of the inner detector trigger performance. The efficiency is shown for both the fast and precision tracking and is significantly better than $99 \%$. The efficiency is observed to be constant as a function of the mean pile-up interaction multiplicity and other variables, with perhaps a slight decrease in efficiency for tracks with a large impact parameter.

Shown in Fig. 18 are the resolutions for the trigger tracks in $\eta, z_{0}$, and $d_{0}$ with respect to the offline muon candidate pseudorapidity on the left, and with respect to the offline muon candidate $p_{\mathrm{T}}$ on the right. The resolution with respect to $\eta$ is shown for the precision tracking and the fast tracking for the same two representative thresholds, $p_{\mathrm{T}}>4 \mathrm{GeV}$ and $20 \mathrm{GeV}$, used in the efficiency determination. The resolution is seen to deteriorate somewhat for larger pseudorapidities, largely due to the larger amount of material through which the muons must pass as they leave the beam pipe and traverse the inner detector. The endcap silicon detectors are arranged perpendicular to the beam to partially ameliorate this, the effect of which can be seen for absolute pseudorapidities larger than 1.2 which is approximately the boundary between the barrel and endcap silicon detectors. 


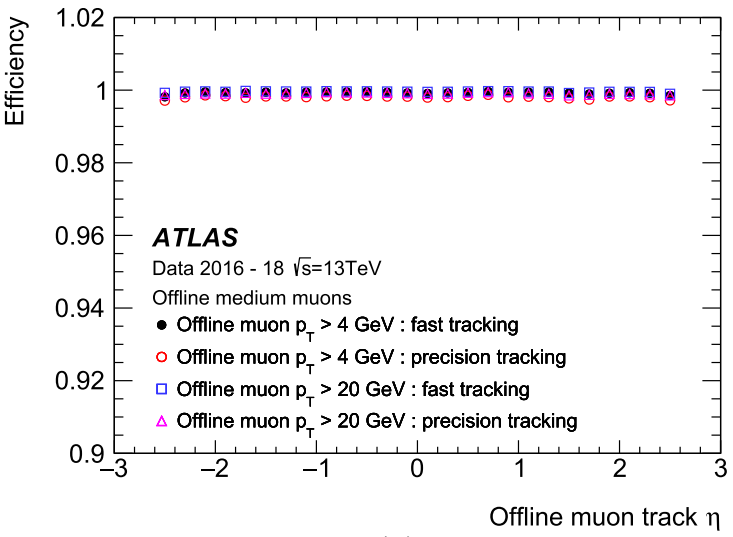

(a)

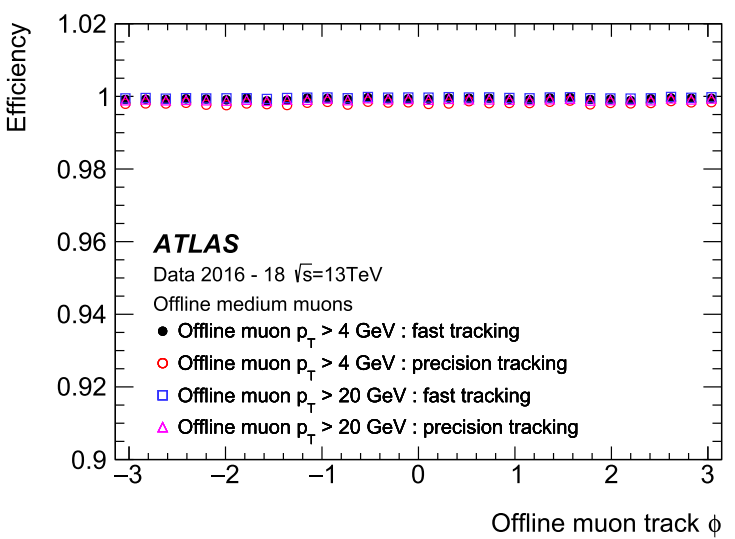

(c)

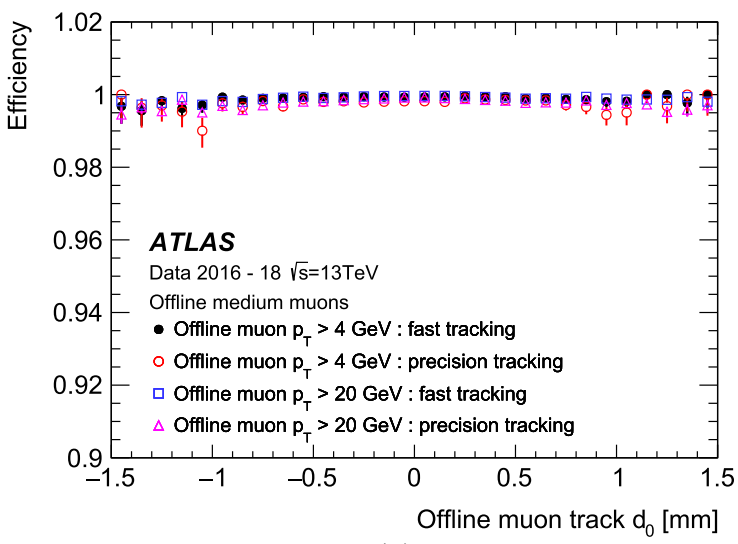

(e)

Fig. 17 The ID tracking efficiency for muons selected by the $4 \mathrm{GeV}$ and $20 \mathrm{GeV}$ muon support triggers, with respect to medium offline muon candidates with $p_{\mathrm{T}}>4 \mathrm{GeV}$ or $p_{\mathrm{T}}>20 \mathrm{GeV}$. The efficiency is shown as a function of: a the offline reconstructed muon $\eta$, $\mathbf{b}$ the offline reconstructed muon $p_{\mathrm{T}}, \mathbf{c}$ the offline reconstructed muon $\phi, \mathbf{d}$

These effects on the resolutions can be further understood by examination of the mean number of hits on the tracks. The mean multiplicity of pixel, SCT and TRT hits on trigger muon candidate tracks which are matched to offline muon

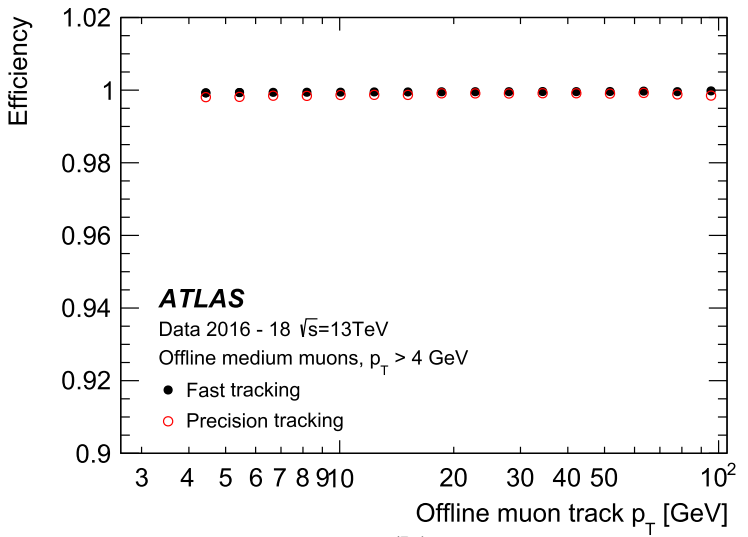

(b)

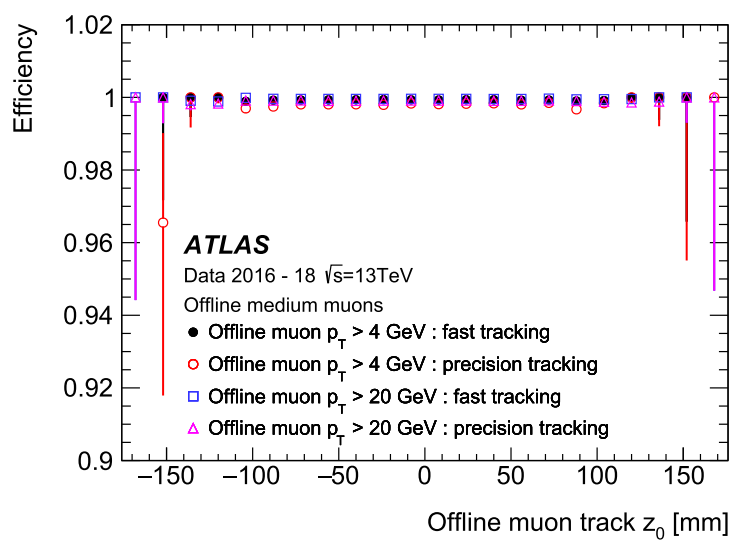

(d)

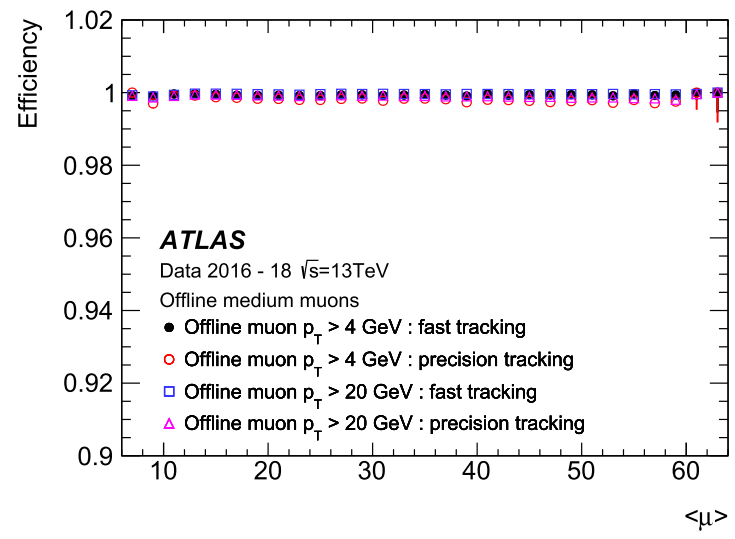

(f)

the offline reconstructed muon $z_{0}$, e the offline reconstructed muon $d_{0}$, and $\mathbf{f}$ the mean number of pile-up interactions, $\langle\mu\rangle$. Efficiencies are shown for both the fast tracking and precision tracking algorithms. Statistical Bayesian uncertainties are shown, using a $68.3 \%$ confidence interval and uniform beta function prior

candidates can be seen in Fig. 19 as a function of the pseudorapidity of the offline muon candidate. For the TRT multiplicity, only the precision tracks are shown since the TRT extension is not performed for the FTF tracks. 


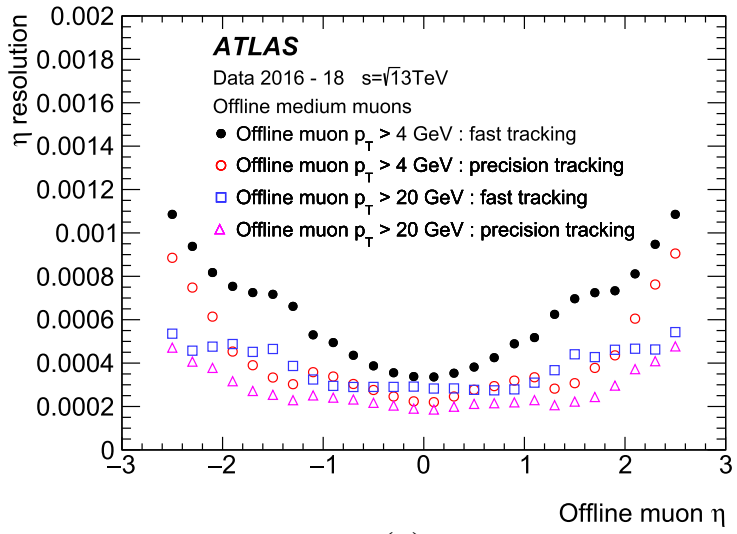

(a)

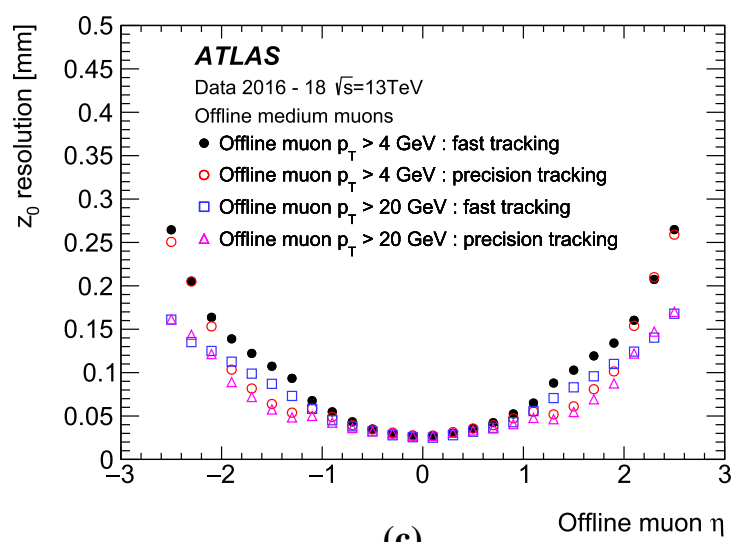

(c)

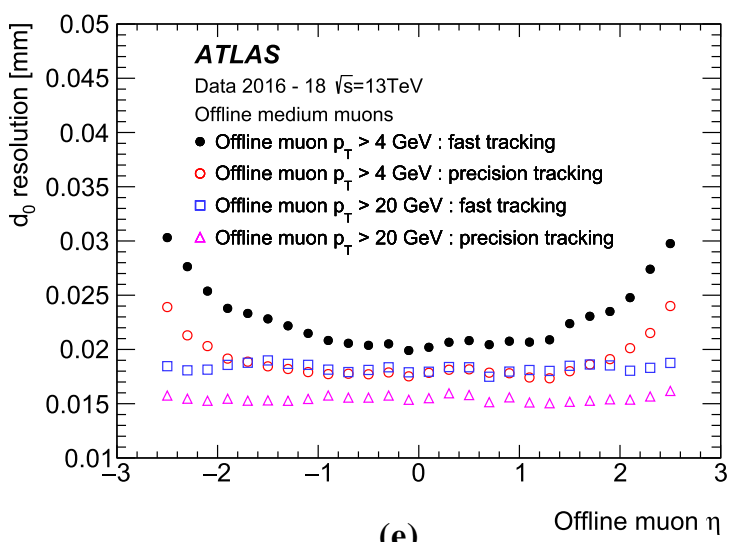

(e)

Fig. 18 The track resolution for pseudorapidity $(\eta)$, track $z$-vertex position, and transverse impact parameter with respect to the beam line, $d_{0}$, as a function of offline track pseudorapidity and transverse momen-

For central pseudorapidities there are approximately four pixel hits per track originating from the four pixel barrel layers. For $|\eta|>1.9$, which delimits approximately the end of the acceptance for the outer pixel barrel layer, the multiplicity increases as the tracks transition into the endcap detectors. It should be noted that the acceptance of the IBL and the innermost of the three original pixel barrel layers extends beyond

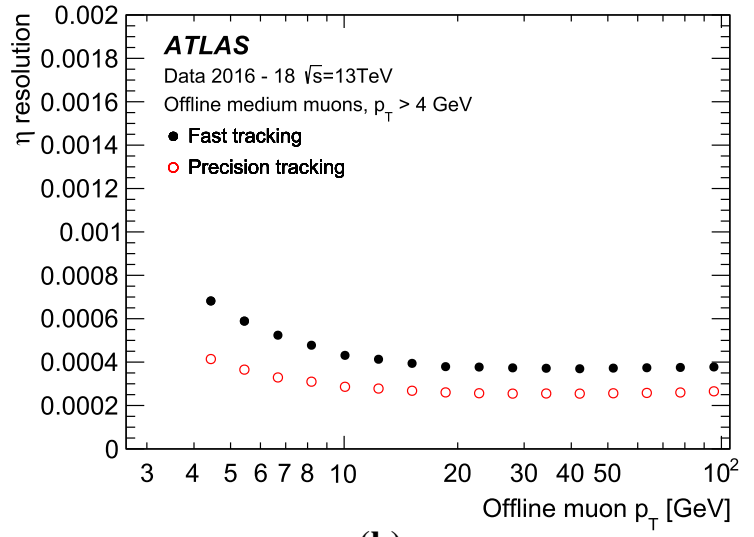

(b)
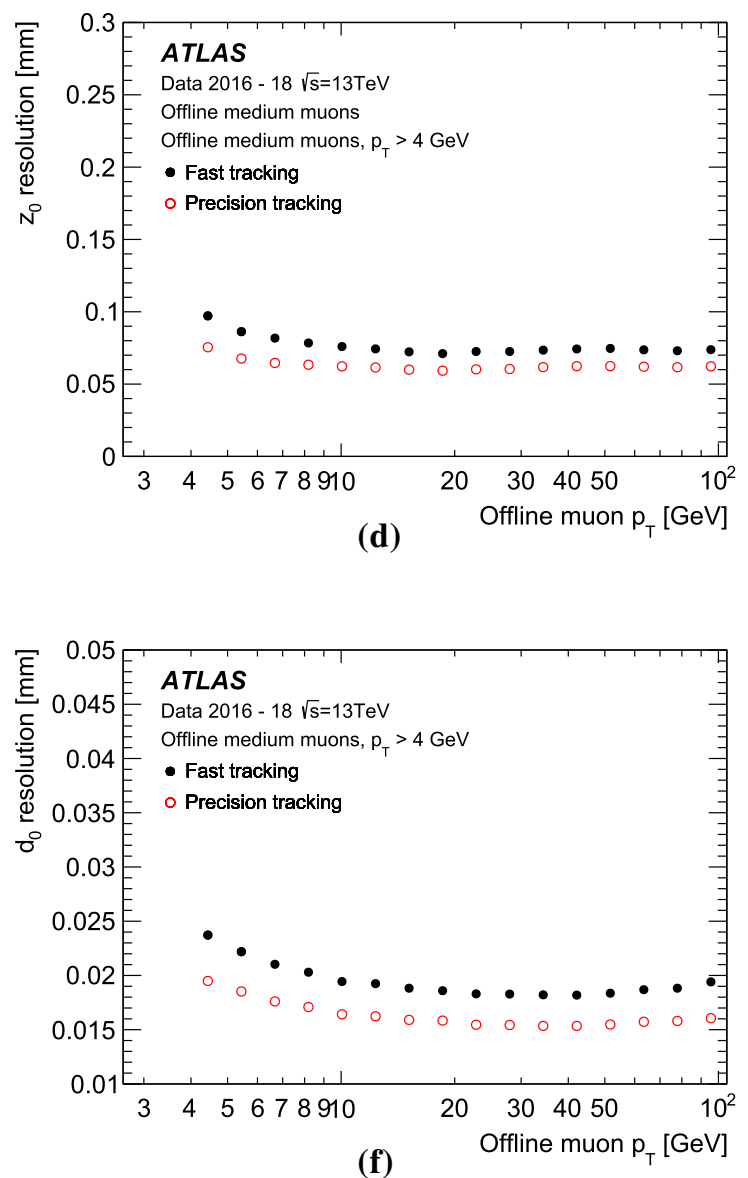

tum. The resolutions are shown for muons selected by the $4 \mathrm{GeV}$ and $20 \mathrm{GeV}$ muon support triggers for both the fast tracking and precision tracking algorithms

$|\eta|=2.5$. This is the reason that the mean pixel cluster multiplicity for tracks at large absolute pseudorapidity is greater than four.

Because of the much larger radius of the outer SCT layer, the transition between barrel and endcap for tracks in the SCT begins much earlier in pseudorapidity, around $|\eta|=1$.2. The four double layers of the barrel are responsible for the mean of 


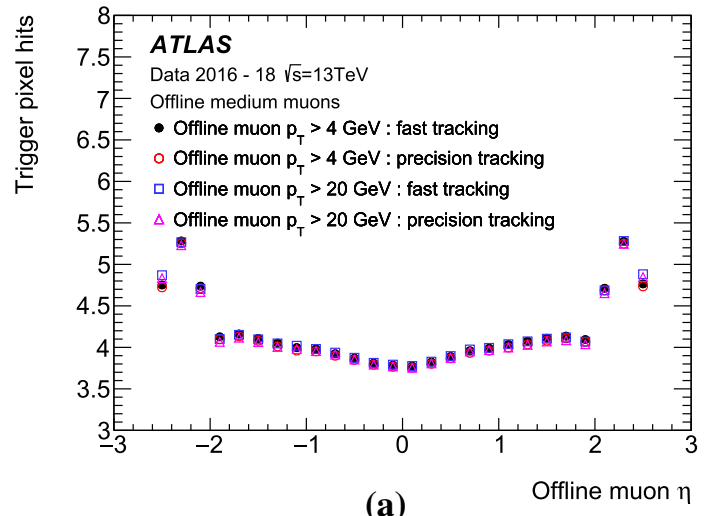

(a)

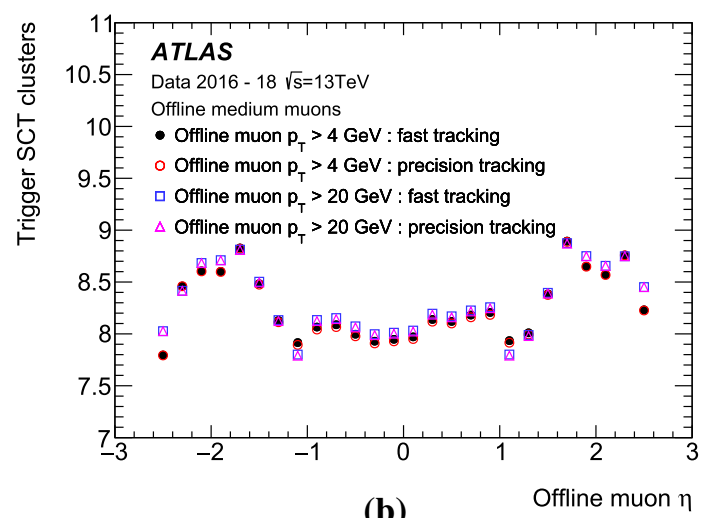

(b)

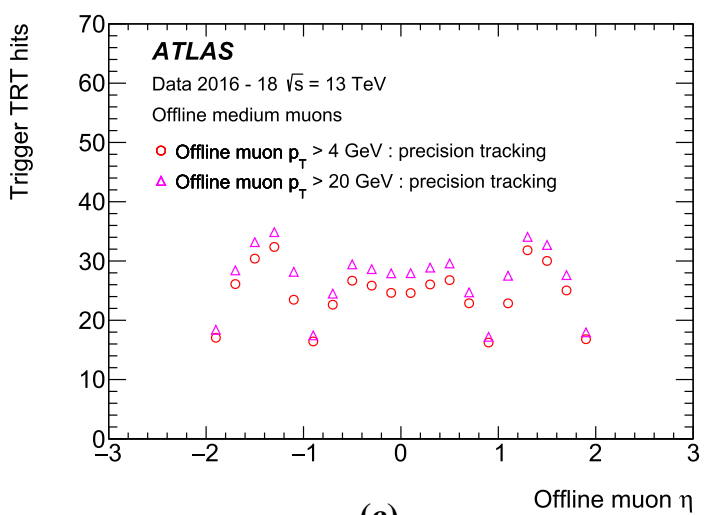

(c)

Fig. 19 The mean multiplicities for a pixel, b SCT, and $\mathbf{c}$ TRT hits on trigger tracks matched to offline tracks as a function of the matching offline track pseudorapidity. Shown are the hit multiplicities for the precision and fast tracking for the pixel and SCT. The TRT extension does not run for the FTF tracks and so the multiplicity for the FTF is not shown in $\mathbf{c}$

eight SCT clusters in the barrel region, and the nine additional wheels in each endcap allow the tracks to maintain a mean of eight of more clusters out to the maximum acceptance at $|\eta|=2.5$. It is largely the transition from SCT barrel to endcap which is responsible for the structure in the resolutions as the tracks transition from pure barrel hits, to a combination of barrel and endcap hits, and finally, to purely endcap

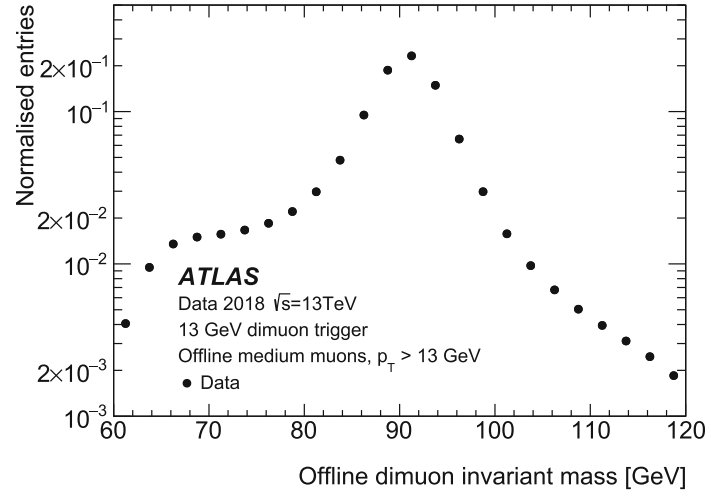

Fig. 20 The offline dimuon invariant mass from events passing the analysis selection from the dimuon performance trigger. For the performance triggers, the RoIs used by the fast and precision tracking are the same and as such the offline dilepton candidates chosen for the analysis of the fast and precision tracking are identical

hits for $|\eta|>1.6$. The small asymmetry between positive and negative pseudorapidities is due to small asymmetries in the detector conditions regarding the detector response and non-operational modules.

The structure of the TRT is somewhat different - there are two barrel sections with only axial straws providing only $r-\phi$ hit information - and two TRT endcaps which provide only $z-\phi$ information. The $z$ position for the end of the TRT barrel is approximately the same as for the SCT barrel, and the endcaps extend to approximately the same maximum $z$ position as the SCT endcaps, such that tracks with $|\eta|>2.1$ have few or no TRT hits. The drop in hit multiplicity for $|\eta|$ around 0.8 is predominantly due to the transition from the barrel to the endcap TRT at $\pm 800 \mathrm{~mm}$ in $z$. A small number of tracks at all pseudorapidities also have no TRT hits. This is more likely for tracks with low transverse momentum, such that the contribution of tracks with no TRT hits reduces the apparent mean hit multiplicity over all tracks and is responsible for the lower mean TRT multiplicity for the lower $p_{\mathrm{T}}$ muon trigger.

In general, the track hit multiplicities are broadly similar for all signatures for full-length tracks. There is a notable exception, however, for electron candidates where the hit multiplicities can be affected by bremsstrahlung. This is discussed in Sect. 5.4.

In addition to the standard performance triggers, which select on tracks reconstructed using the muon spectrometer only, there are additional dimuon performance triggers. These select events with two muon candidates consistent with both muons arising from the decay of a $Z$ boson, by selecting candidates with a dimuon mass within a window around $91 \mathrm{GeV}$ with the range $70<m_{l l}<110 \mathrm{GeV}$. For these triggers, the two muons must each be in a different RoI. One of the RoI - the tag RoI - selects on the full muon candidate including the full selection on the combined spec- 
trometer and inner detector muon candidate, and the other the probe RoI - selects solely on the muon candidate reconstructed in the spectrometer, without using any inner detector information. Selecting on the combined muon candidate in the tag RoI increases the purity of the selected events. The spectrometer-only selection in the probe RoI allows the efficiency to be measured for the probe muon candidate, unbiased by the track reconstruction in the inner detector itself. The presence of the first, reasonably pure fully selected tag muon reduces the rate of such events and the selection of the two muon candidates having to form a $Z$ boson candidate greatly enhances the purity of the spectrometer-only probe muon candidates, particularly at high transverse momenta.

For the fully selecting, tag leg, the muon trigger selects muon candidates with $p_{\mathrm{T}}>13 \mathrm{GeV}$. The probeleg, selecting on the muon candidates reconstructed in the spectrometer only, also selects candidates with transverse momenta greater than $13 \mathrm{GeV}$. Unbiased measurements are therefore possible from these triggers for muon transverse momenta greater than $13 \mathrm{GeV}$. The data used for this analysis correspond to the full 2018 integrated luminosity.

The dilepton invariant mass distribution for offline candidates for the events passing the medium offline muon selection, with no additional background rejection, for the dimuon performance trigger but with no corrections for trigger efficiency or detector response can be seen in Fig. 20.

The efficiency as a function of the mean pile-up interaction multiplicity, $\langle\mu\rangle$, and the offline track variables can be seen in Fig. 21. With this analysis, the statistical uncertainties are very much reduced with respect to the analyses presented earlier in this section. The efficiency for both the fast and precision tracking can be seen to approach $100 \%$ with small statistical uncertainties even at large transverse momenta, approaching $1 \mathrm{TeV}$. The efficiency versus the transverse impact parameter clearly shows a very small decrease of slightly less than $0.5 \%$ for the fast tracking for $d_{0}$ values of $1.5 \mathrm{~mm}$, and slightly larger than $0.5 \%$ for the precision tracking. This is consistent with the suggestion from Fig. 17. The efficiency for both the precision and fast tracking is again seen to be approximately constant with respect to changes in the $z_{0}$ position, but with a very small loss of efficiency for the precision tracking at high pile-up interaction multiplicity consistent with Fig. 17.

The resolution in the track pseudorapidity, track $z$ position along the beam line, and the transverse impact parameter for these data are shown in Fig. 22 as functions of the offline track pseudorapidity and transverse momentum. The performance of the resolution at large transverse momentum, with the exception of $d_{0}$, is seen to be generally consistent with that seen in Fig. 18 but again with significantly smaller uncertainties, most notably at large track transverse momenta. With regards to the resolution in $d_{0}$, the dilepton analysis is performed using only the 2018 data where the mean pile- upinteraction multiplicity is larger than for the combined 2016-2018 data. Since the transverse impact parameter resolution has some dependence on the pile-up interaction multiplicity, the resolution seen in Fig. 22 is somewhat worse than that shown in Fig. 18 for the combined 2016-2018 data.

\subsection{Tracking in the electron signature}

Because of the significant background from QCD jet events, a tight offline likelihood identification [11] is used for the reference electron selection for the electron signature analysis. The offline electron candidate track is required to have at least two pixel hits, an IBL hit if passing through at least one active IBL module, and at least four clusters in the SCT. These requirements were chosen to ensure better reconstruction in the pixel detector for offline tracks by eliminating extremely poorly reconstructed bremsstrahlung candidates where the hits in the pixel detector were missed. Analyses for several triggers are presented, each employing a kinematic selection of $|\eta|<2.5$ and $p_{\mathrm{T}}>5 \mathrm{GeV}$ for the offline electron candidate tracks. In addition, there is a selection on the electron candidate transverse energy, $E_{\mathrm{T}}$. For the candidates from the $5 \mathrm{GeV}$ trigger a selection of $E_{\mathrm{T}}>5 \mathrm{GeV}$ is applied. For the $10 \mathrm{GeV}$ trigger the selection is $E_{\mathrm{T}}>10 \mathrm{GeV}$, and $E_{\mathrm{T}}>26 \mathrm{GeV}$ for the $26 \mathrm{GeV}$ trigger. For the analyses with the harder $E_{\mathrm{T}}$ selection, the same $5 \mathrm{GeV}$ track $p_{\mathrm{T}}$ selection is used. Consequently, the fraction of the electron candidate energy that can be radiated as bremsstrahlung for candidates near the trigger threshold for the higher $E_{\mathrm{T}}$ threshold triggers is larger than for the lower $E_{\mathrm{T}}$ threshold triggers. As an additional requirement, to remove offline candidates where the track $p_{\mathrm{T}}$ has been badly overestimated, offline candidates with $E_{\mathrm{T}} / p_{\mathrm{T}}<0.8$ are removed, where $E_{\mathrm{T}}$ is measured in the calorimeter, and $p_{\mathrm{T}}$ is from the offline track.

Figure 23 shows the ID tracking efficiency for the $5 \mathrm{GeV}$ and $26 \mathrm{GeV}$ electron triggers as a function of the $\eta, \phi$, and $z_{0}$ values of the offline electron candidate track, and as a function of the mean pile-up multiplicity. The tracking efficiency is measured with respect to offline electron tracks with $p_{\mathrm{T}}>5 \mathrm{GeV}$ for trigger electron candidates with either $E_{\mathrm{T}}>5 \mathrm{GeV}$ or $E_{\mathrm{T}}>26 \mathrm{GeV}$, from the $5 \mathrm{GeV}$ and $26 \mathrm{GeV}$ electron support triggers. The offline electro tracks are those from tight offline electron candidates. The efficiencies of the fast track finder and precision tracking exceed $99 \%$ for all pseudorapidities.

Figure 24 shows the resolutions of the track pseudorapidity and $1 / p_{\mathrm{T}}$ as a function of either the $\eta$ or $p_{\mathrm{T}}$ of the offline track from the offline electron candidates. For this analysis, the same selection on the electron candidate $E_{\mathrm{T}}$ and $p_{\mathrm{T}}$ discussed previously is applied. Consequently, for events with a $5 \mathrm{GeV}$ selection, there is no phase space for the electron candidates near the threshold to radiate any bremsstrahlung photons. For the $26 \mathrm{GeV}$ electrons however, there is significant 


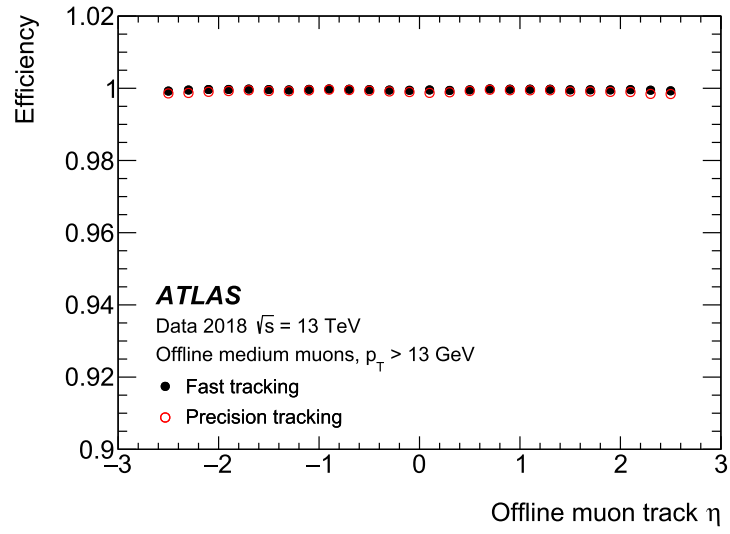

(a)

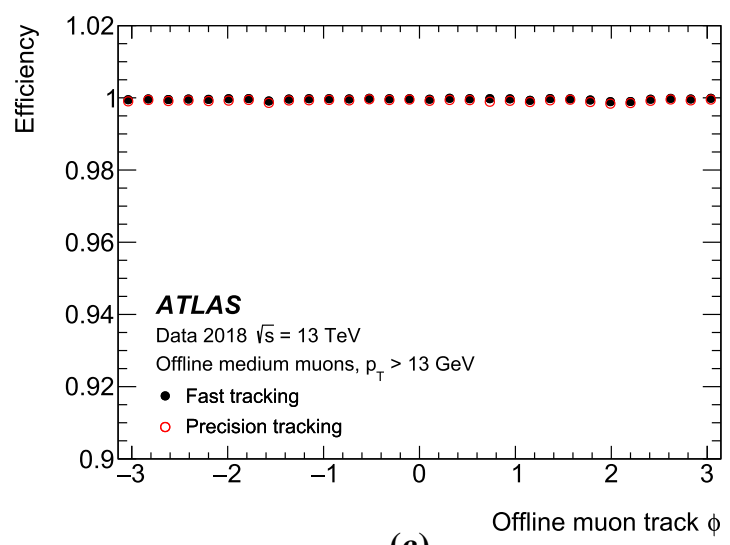

(c)

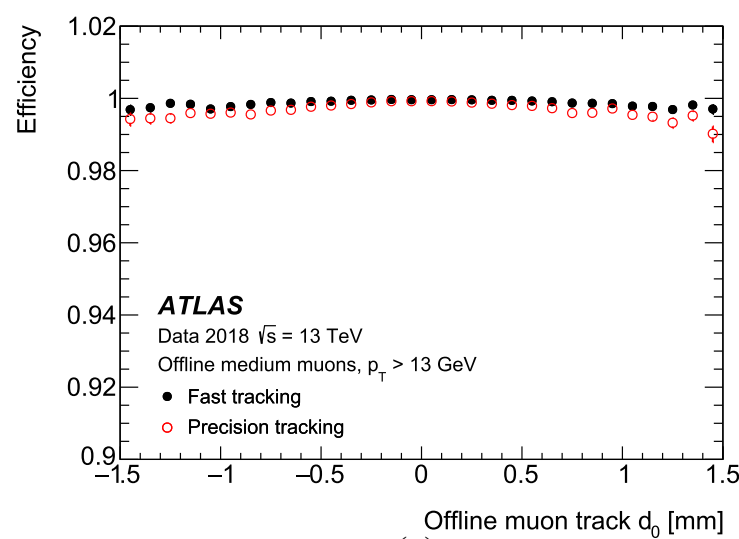

(e)

Fig. 21 The muon track finding efficiency versus a the offline muon track pseudorapidity, $\mathbf{b}$ the offline muon track transverse momentum, $\mathbf{c}$ the offline muon track azimuthal angle, $\mathbf{d}$ the offline muon track $z_{0}$ position along the beam line, $\mathbf{e}$ the offline muon track transverse impact

phase space available for radiation. As such, for the $26 \mathrm{GeV}$ threshold trigger, tracks which have $p_{\mathrm{T}}$ far below $26 \mathrm{GeV}$ will correspond to electrons that have undergone significant radiation. The resolutions for these tracks will be significantly worse than for the tracks with the same $p_{\mathrm{T}}$ from the $5 \mathrm{GeV}$

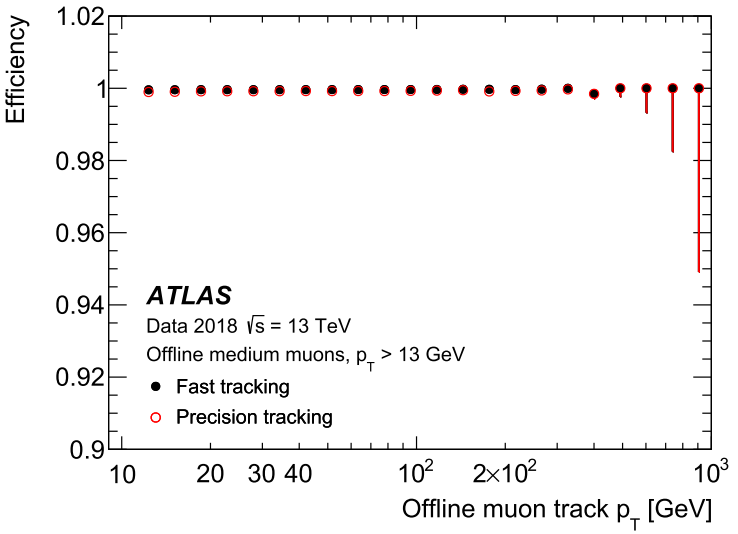

(b)

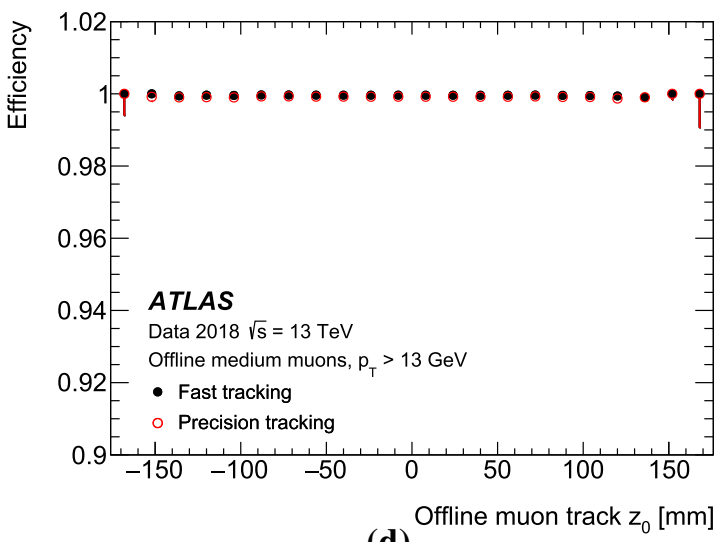

(d)

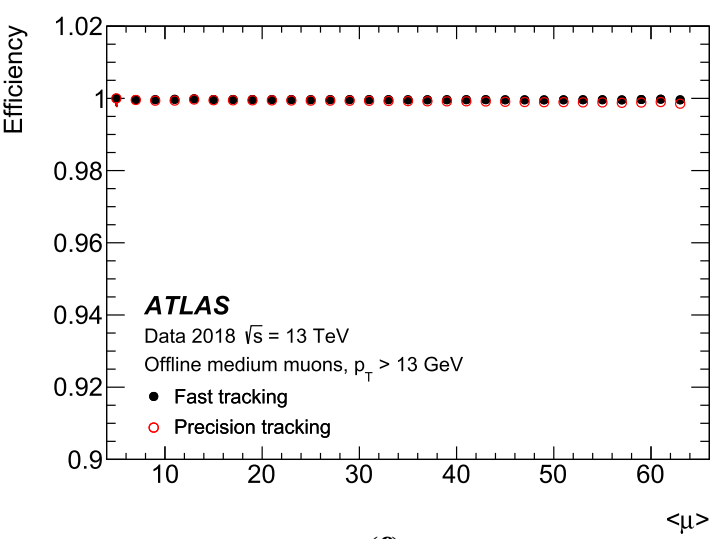

(f)

parameter, $d_{0}$, measured with respect to the beam line, and $\mathbf{f}$ the mean pile-up interaction multiplicity, all measured using the dimuon performance trigger. Statistical Bayesian uncertainties are shown, using a $68.3 \%$ confidence interval and uniform beta function prior

trigger, but that have not undergone such emission. For the $5 \mathrm{GeV}$ threshold, the resolution of the inverse $p_{\mathrm{T}}$ for the fast tracking is slightly worse than for the precision tracking. As expected it is seen to deteriorate at larger $\eta$ for both the higher and lower threshold triggers. Despite the significantly worse 


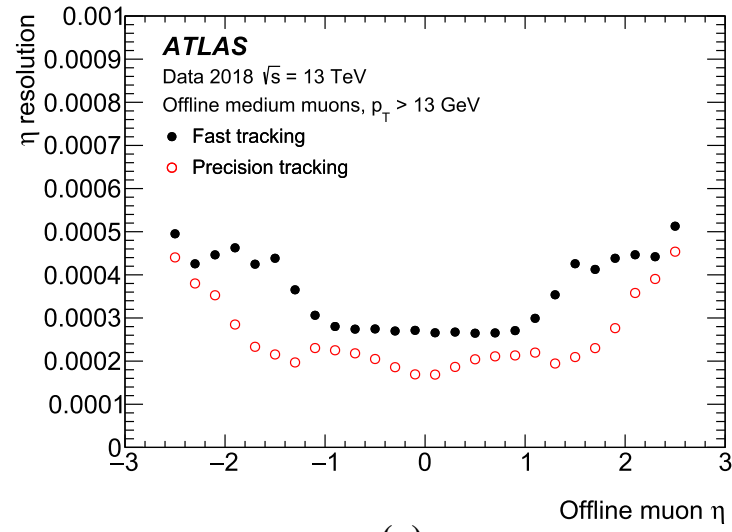

(a)

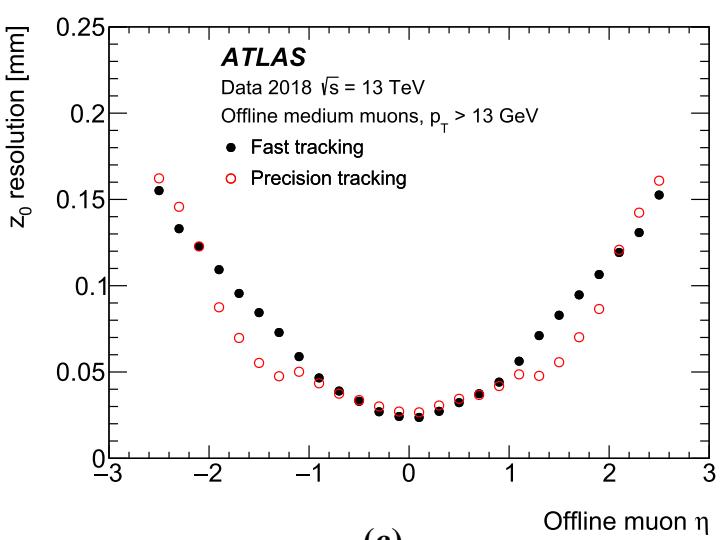

(c)

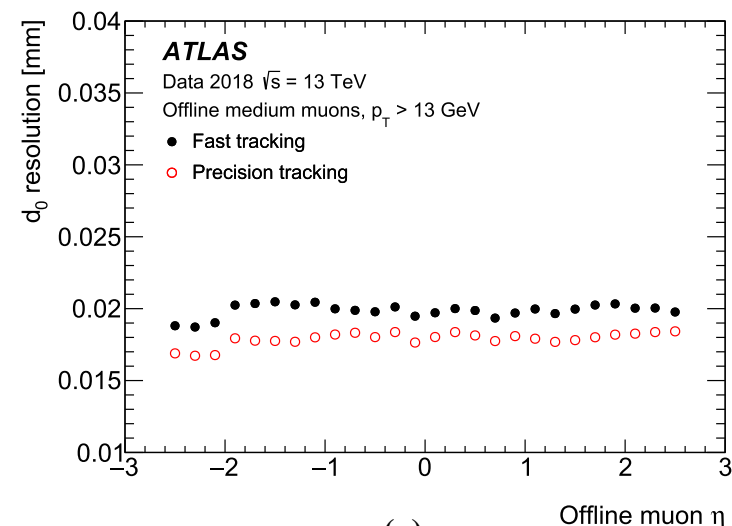

(e)

Fig. 22 The resolution in the pseudorapidity and inverse transverse momentum as a function of offline track pseudorapidity and transverse momentum. The resolutions are shown for muons selected with the

resolution at low $p_{\mathrm{T}}$ for the tracks from the $26 \mathrm{GeV}$ trigger, the resolution, when integrated over $p_{\mathrm{T}}$, is still significantly better than for the lower $p_{\mathrm{T}}$ threshold since only a relatively small fraction of events from the $26 \mathrm{GeV}$ trigger have low $p_{\mathrm{T}}$ tracks. Therefore, the usual improvement of the resolu-

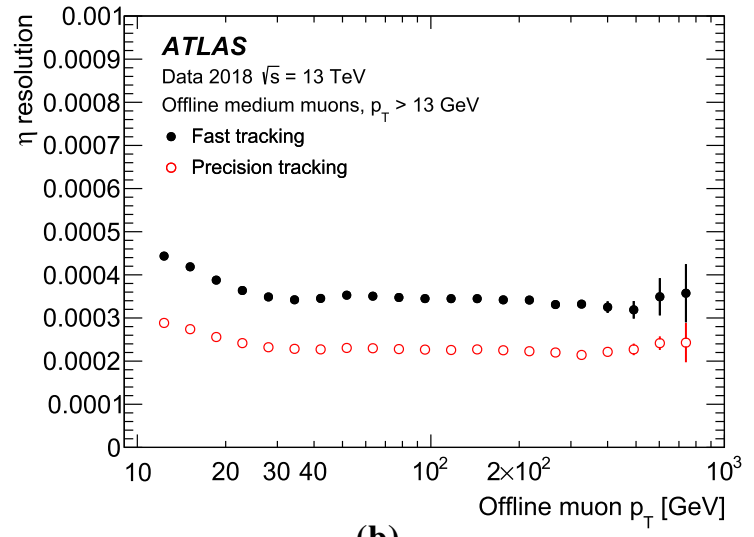

(b)
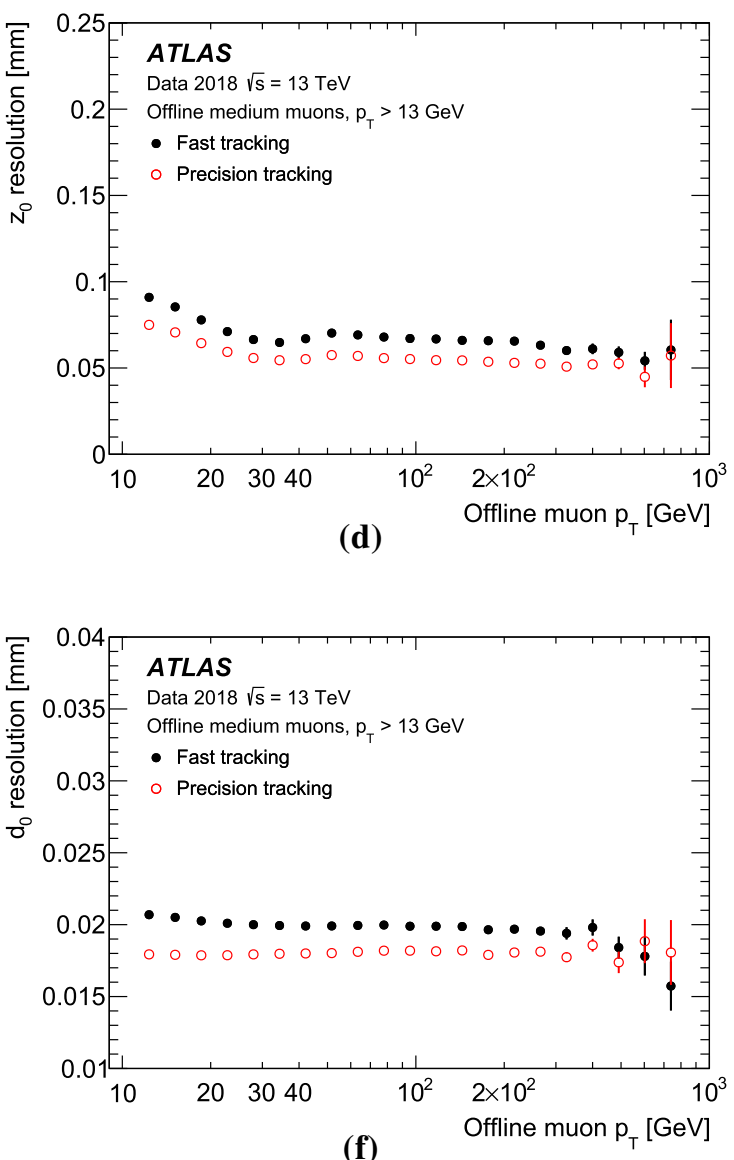

$p_{\mathrm{T}}>15 \mathrm{GeV}$ muon requirement from the dimuon support triggers, for both the fast tracking and precision tracking algorithms

tion with increasing $p_{\mathrm{T}}$ is enough to provide the observed significantly better overall resolution.

The resolution for the precision tracking at low $p_{\mathrm{T}}$ and large pseudorapidities from the $26 \mathrm{GeV}$ trigger is worse than for the fast tracking as a consequence of the different SCT 


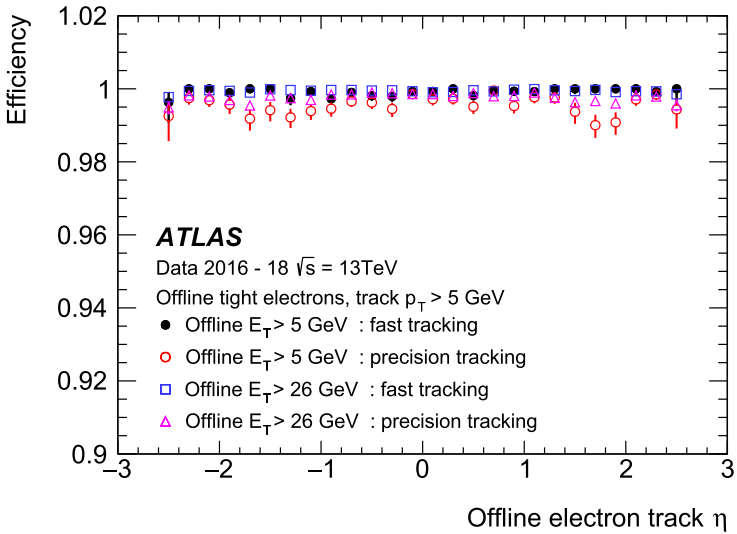

(a)

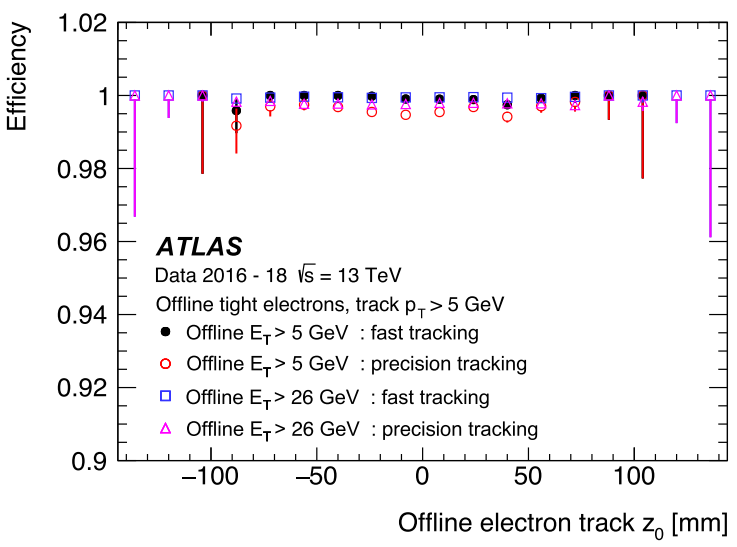

(c)

Fig. 23 The ID trigger tracking efficiency for electrons selected by the $5 \mathrm{GeV}$ and $26 \mathrm{GeV}$ electron triggers, with respect to offline electron candidates with $E_{\mathrm{T}}>5 \mathrm{GeV}$ for the $5 \mathrm{GeV}$ trigger and $E_{\mathrm{T}}>26 \mathrm{GeV}$ for the $26 \mathrm{GeV}$ trigger. The efficiency is shown as a function of a the offline reconstructed electron track $\eta$, $\mathbf{b}$ the offline reconstructed elec-

hit multiplicities observed on each type of trigger track. This is discussed in more detail later in this section.

This difference between the degree of bremsstrahlung for tracks between 5 and $26 \mathrm{GeV}$ from the different triggers is also responsible for the differences in the efficiency as a function of the track $p_{\mathrm{T}}$ observed in Fig. 25. For this figure the $E_{\mathrm{T}} / p_{\mathrm{T}}$ selection is relaxed. Here, it is clear that the track finding efficiency for all electron candidates as a function of the $E_{\mathrm{T}}$ of the offline electron candidate is consistently high. For the track candidates from the $26 \mathrm{GeV}$ trigger but with tracks with $p_{\mathrm{T}}$ below $26 \mathrm{GeV}$ there is significant radiation, and the track reconstruction is less efficient, as there may be significant kinks in the electron trajectory. Also shown is the distribution of the ratio of the offline electron transverse energy measured in the calorimeter to the offline track transverse momentum. The long tail to values greater than unity represents bremsstrahlung candidates. Also seen in the

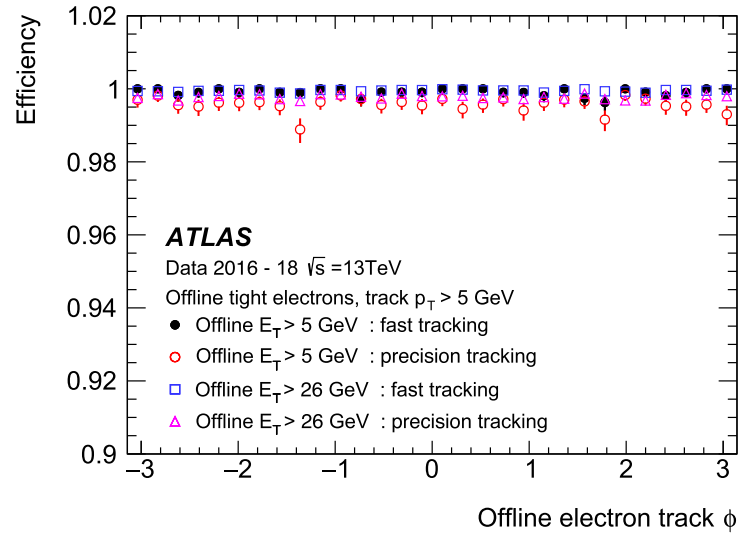

(b)

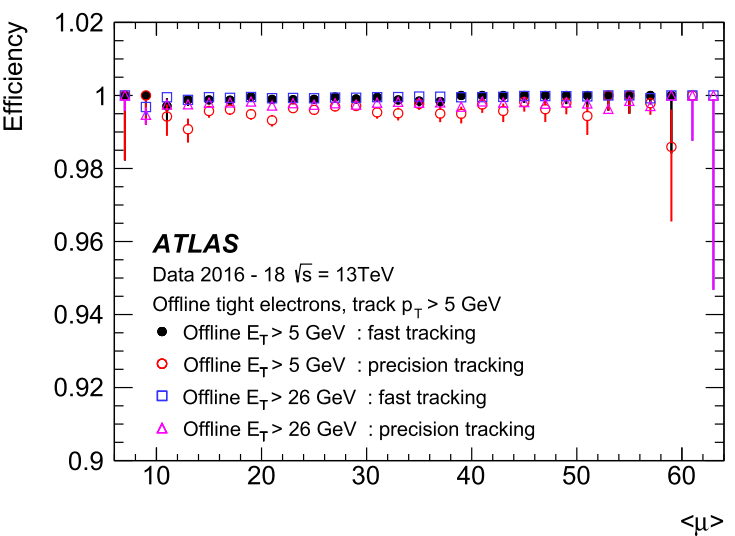

(d)

tron track $\phi, \mathbf{c}$ the offline reconstructed electron $z_{0}, \mathbf{d}$ the mean number of pile-up vertices, $\langle\mu\rangle$. Efficiencies are shown for both the fast tracking and precision tracking algorithms. Statistical Bayesian uncertainties are shown, using a $68.3 \%$ confidence interval and uniform beta function prior

figure is the clear tail with values of $E_{\mathrm{T}} / p_{\mathrm{T}}$ less than one, which results from electron candidates where the track $p_{\mathrm{T}}$ is larger than the cluster $E_{\mathrm{T}}$ from the calorimeter. This is a consequence of the offline $p_{\mathrm{T}}$ measurement migrating to larger values when the track $p_{\mathrm{T}}$ itself is less well reconstructed. In this case, the track cannot realistically have significantly more energy than the cluster, and so any larger $p_{\mathrm{T}}$ value would therefore represent a less reliable track $p_{\mathrm{T}}$ measurement. The efficiency as a function of $E_{\mathrm{T}} / p_{\mathrm{T}}$ shows a clear decrease, with a loss of around $1 \%$ for tracks where the track $p_{\mathrm{T}}$ is $60 \%$ higher than might be physically possible. The efficiency for tracks which have not radiated is well over $99 \%$, and the efficiency for tracks that have radiated well over half of their original energy is still greater than $98 \%$.

The selection on $E_{\mathrm{T}} / p_{\mathrm{T}}>0.8$ is not strictly required for measurements which integrate over the transverse momentum of the tracks, but is essential for an unbiased measure- 


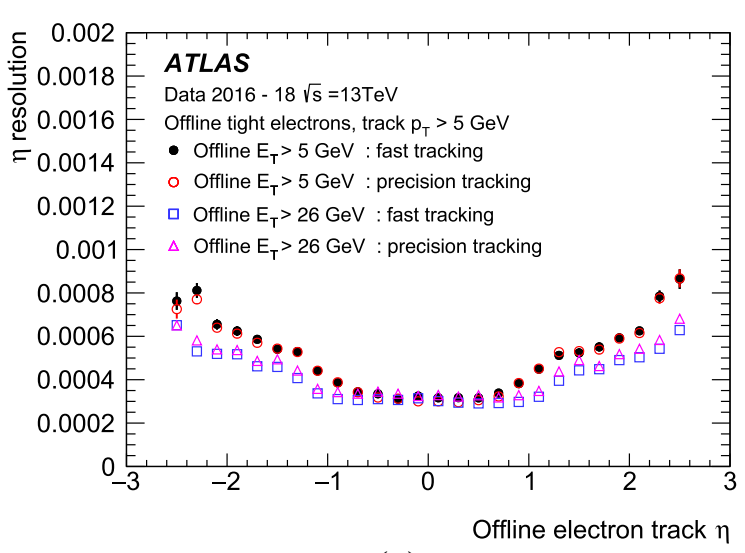

(a)

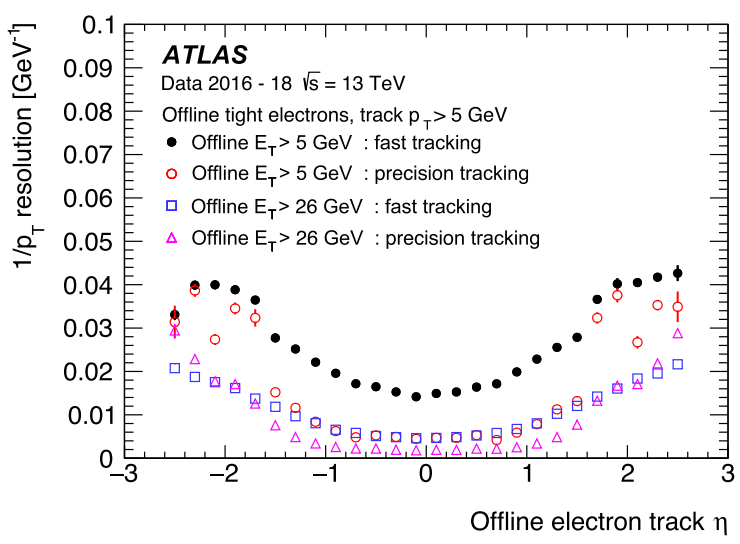

(c)

Fig. 24 The resolution for pseudorapidity and inverse transverse momentum as a function of offline track pseudorapidity and transverse momentum. The resolutions are shown for

ment versus the offline track $p_{\mathrm{T}}$ itself. Therefore, for consistency this selection is applied everywhere, with the exception of the study of the $E_{\mathrm{T}} / p_{\mathrm{T}}$ variable and the corresponding efficiency.

To complement the standard performance triggers, which do not select on the tracking, as with the muon trigger, there are additional dilepton performance triggers in the electron signature which select events with two electron candidates consistent with both electrons originating from the decay of a $Z$ boson. This allows a tag and probe analysis largely similar to that discussed in Sect. 5.3. For these triggers, one of the electrons is fully selected - using the full selection including the tracking information to increase the purity of the selected candidates - and the selection of the other electron is based solely on the calorimeter reconstruction in the trigger.

This allows the efficiency of the tracking to again be measured for the electron candidate selected using only the calorimeter reconstruction, unbiased by the track selection itself. The presence of the first, reasonably pure fully selected

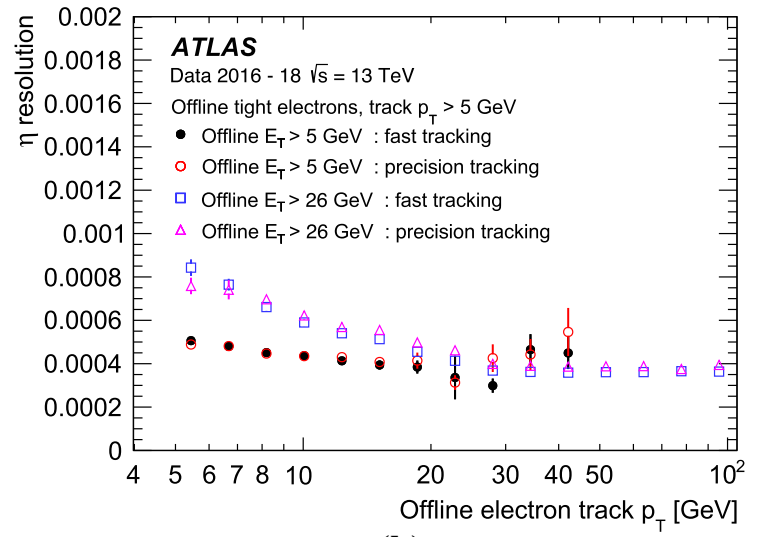

(b)

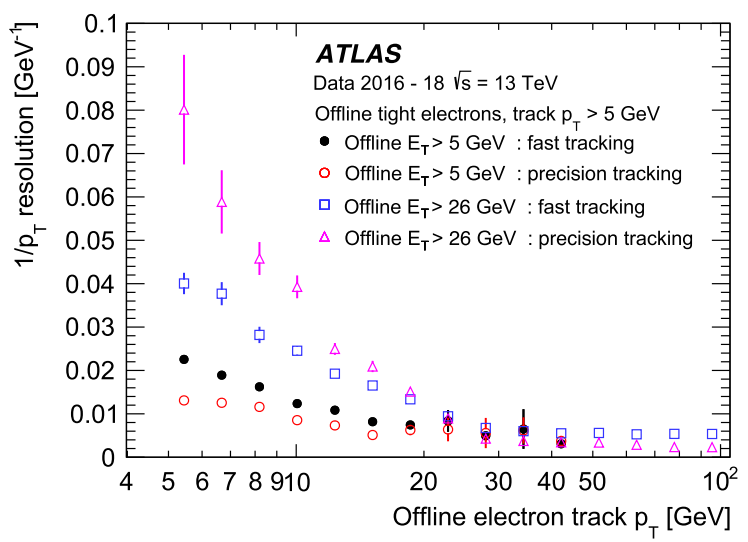

(d)

electrons selected by the $5 \mathrm{GeV}$ and $26 \mathrm{GeV}$ electron support triggers for both the fast tracking and precision tracking algorithms

tag electron reduces the rate of events with two electron candidates and the selection of the two electron candidates having to form a $Z$ boson candidate greatly enhances the purity of the calorimeter-only probe electron candidate. The purity of electron candidates in such events from these triggers is therefore high and the triggers themselves have a reasonably high rate. However, because the triggers are limited to $Z$ candidates, and have a reasonably high threshold for the electon selection in the calorimeter, they do not have the same full range in transverse energy as the standard performance triggers.

For the following analysis such a double-electron performance trigger which selects on a $Z$ boson candidate is used, with the fully selected electron candidate passing the $E_{\mathrm{T}}>26 \mathrm{GeV}$ trigger. In this case the calorimeter-only probe electron candidate is required to have $E_{\mathrm{T}}>15 \mathrm{GeV}$. As such, unbiased measurements are possible from this trigger for electron candidates above this $15 \mathrm{GeV}$ threshold. The data used for this analysis correspond to the full 2018 integrated luminosity. 


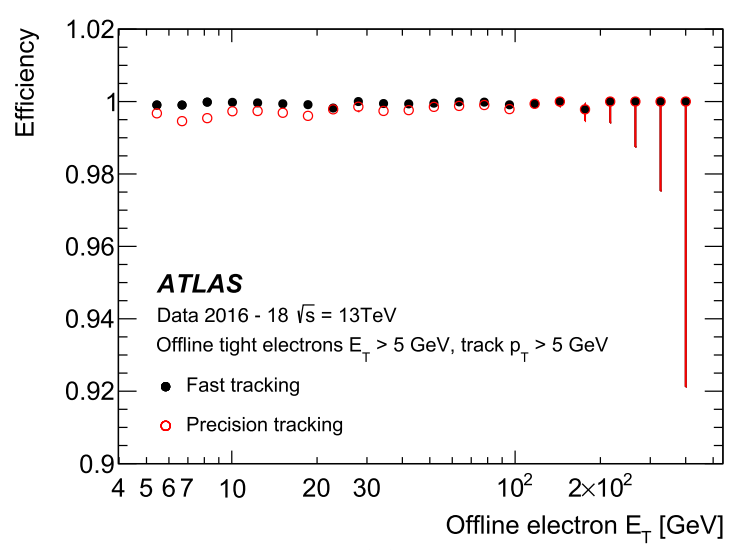

(a)

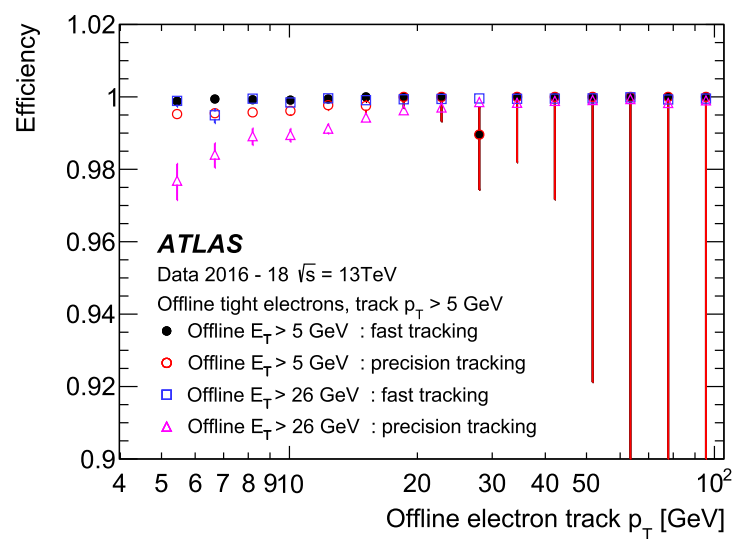

(c)

Fig. 25 The efficiency with respect to a the offline electron transverse energy, $E_{\mathrm{T}}$, b the offline electron $E_{\mathrm{T}} / p_{\mathrm{T}}$, and $\mathbf{c}$ the offline electron track $p_{\mathrm{T}}$. Shown in $\mathbf{d}$ is the distribution of $E_{\mathrm{T}} / p_{\mathrm{T}}$ for the offline electron candidates. Results are shown for electrons selected by the $5 \mathrm{GeV}$, $10 \mathrm{GeV}$ and $26 \mathrm{GeV}$ electron support triggers for panel a, and by the

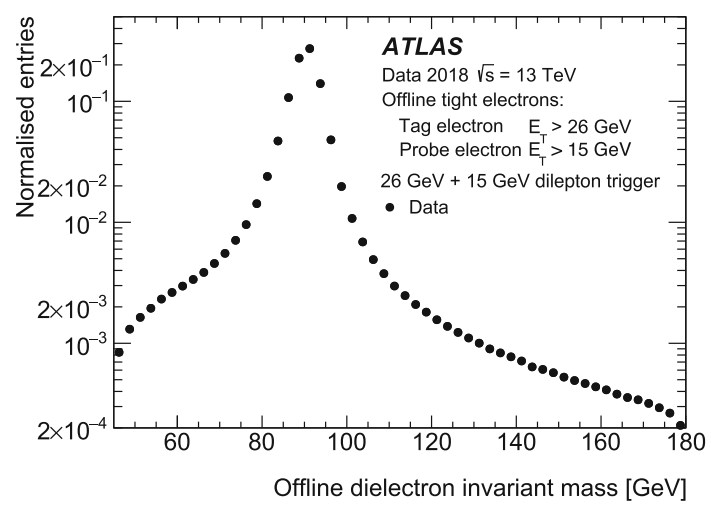

Fig. 26 The uncorrected offline dilepton invariant mass in events from the dilepton performance trigger that pass the analysis selection. For the performance triggers, the RoIs used by the fast and precision tracking are the same and so the offline dilepton candidates chosen for the analysis of the fast and precision tracking are identical

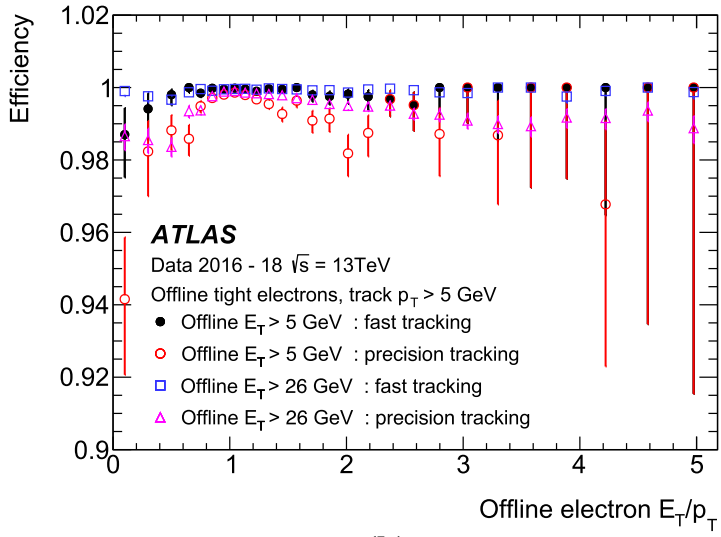

(b)

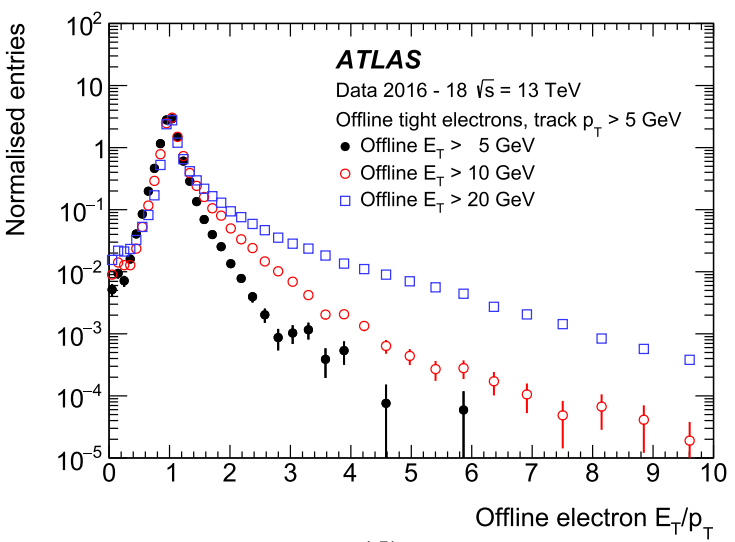

(d)

$5 \mathrm{GeV}, 26 \mathrm{GeV}$ support triggers for panels $\mathbf{b}$ and $\mathbf{c}$. For the efficiencies versus offline electron candidate $E_{\mathrm{T}}$ and $p_{\mathrm{T}}$, only candidates where $E_{\mathrm{T}} / p_{\mathrm{T}}>0.8$ are used. Statistical Bayesian uncertainties are shown, using a $68.3 \%$ confidence interval and uniform beta function prior

The dilepton invariant mass distribution for offline candidates for the events passing the offline selection for the combined $26 \mathrm{GeV}$ and $15 \mathrm{GeV}$ dilepton performance trigger but with no corrections for trigger efficiency or detector response can be seen in Fig. 26.

Figure 27 shows the efficiency for the offline electron candidates versus the offline electron candidate $E_{\mathrm{T}}$ and track $p_{\mathrm{T}}$, and versus the ratio $E_{\mathrm{T}} / p_{\mathrm{T}}$, together with the $E_{\mathrm{T}} / p_{\mathrm{T}}$ distribution itself, determined using the tag and probe trigger. The reduction of the efficiency for candidates with significantly overestimated track transverse momenta can again be seen but with higher statistical precision. For the remainder of the analysis, those candidates with $E_{\mathrm{T}} / p_{\mathrm{T}}<0.8$ are once again removed to avoid biases from the track $p_{\mathrm{T}}$ measurement at high track $p_{\mathrm{T}}$.

The efficiency as a function of $E_{\mathrm{T}} / p_{\mathrm{T}}$ is seen to behave in a manner consistent with that from the inclusive analyses presented earlier in this section, but now with greatly reduced 


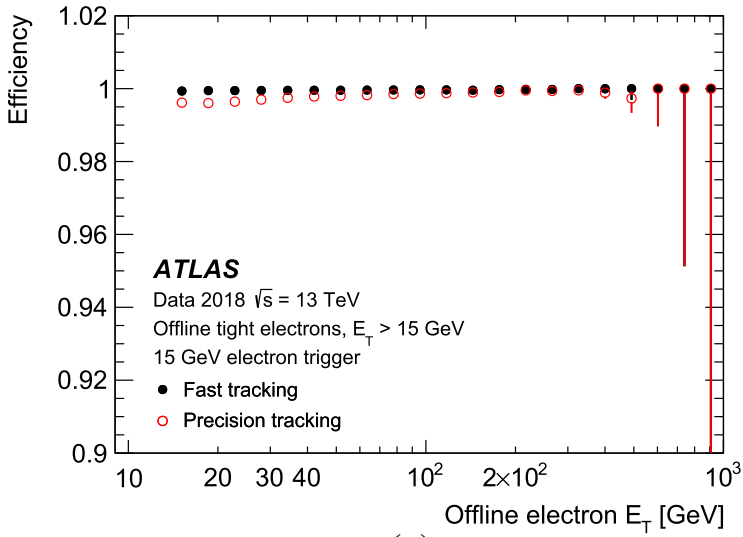

(a)

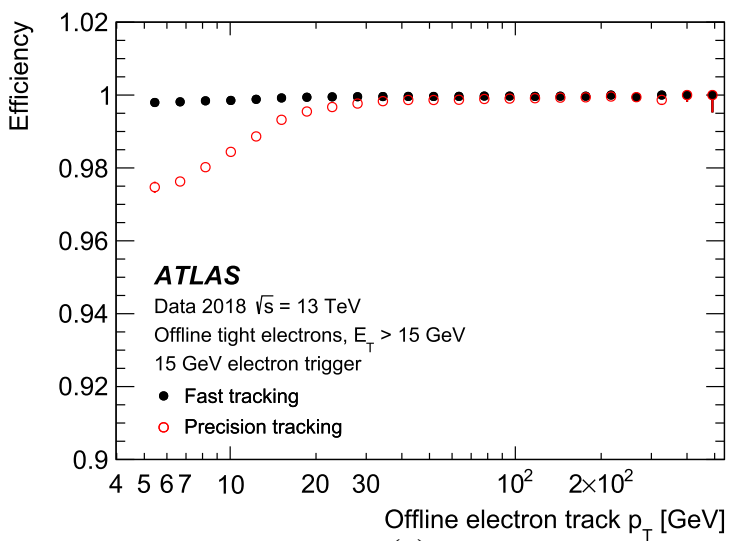

(c)

Fig. 27 The efficiency versus transverse energy of the electron candidate from the tag and probe analysis of the $15 \mathrm{GeV}$ trigger with respect to $\mathbf{a}$ the offline electron transverse momentum, $\mathbf{b}$ the offline electron $E_{\mathrm{T}} / p_{\mathrm{T}}$, and $\mathbf{c}$ the offline electron track $p_{\mathrm{T}}$. Shown in $\mathbf{d}$ is the distri-

statistical uncertainties. It is reassuring to see that the efficiency for the fast tracking remains very high for all such candidates, and is still seen for the precision tracking to be higher than $98 \%$ even for candidates that have radiated up to $80 \%$ of their energy.

The efficiency as a function of $E_{\mathrm{T}}$ remains very high even for very high energies up to $1 \mathrm{TeV}$ seen here, with a reduction of only approximately $0.5 \%$ near the $15 \mathrm{GeV}$ trigger threshold. The efficiency versus the track $p_{\mathrm{T}}$ is also constant at large $p_{\mathrm{T}}$, as seen in the earlier analyses. Candidates with $p_{\mathrm{T}}$ between $5 \mathrm{GeV}$ and $15 \mathrm{GeV}$ consist almost entirely of candidates that have undergone significant bremsstrahlung and yet are still reconstructed with efficiencies greater than $97 \%$ for the precision tracking, and better than $99.5 \%$ for the fast tracking. It should be stressed that in this region between $5 \mathrm{GeV}$ and $15 \mathrm{GeV}$, it is not that the overall electron tracking efficiency is reduced, but rather that the selection on the electron candidate $E_{\mathrm{T}}$ is acting to preferentially reject any candidate that has not radiated. This efficiency for candi-

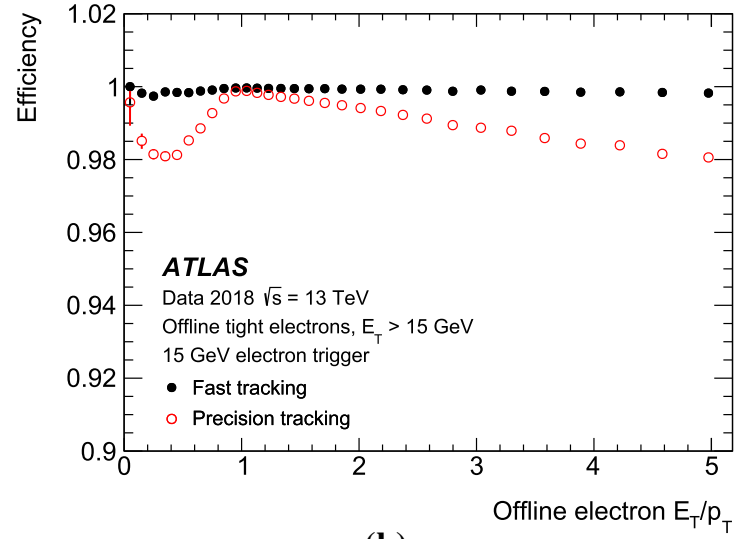

(b)

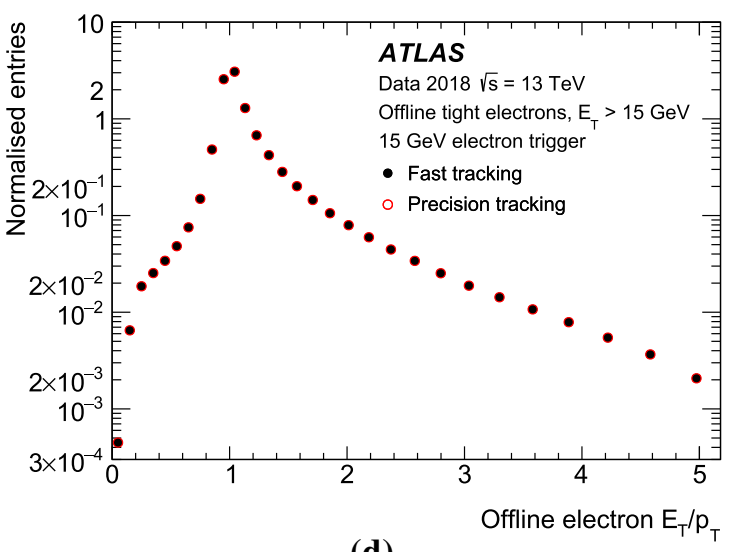

(d)

bution of $E_{\mathrm{T}} / p_{\mathrm{T}}$ for the offline electron candidates. For the efficiencies versus offline electron candidate $E_{\mathrm{T}}$ and $p_{\mathrm{T}}$, only candidates with $E_{\mathrm{T}} / p_{\mathrm{T}}>0.8$ are used

dates with transverse momenta below $15 \mathrm{GeV}$ therefore represents that only for those candidates that have undergone bremsstrahlung.

The efficiency with respect to the offline track pseudorapidity and $z$ position along the beam line, together with the efficiency versus the mean pile-up interaction multiplicity can be seen in Fig. 28. The efficiency versus pseudorapidity shows a clear $0.5 \%$ inefficiency for the precision tracking with pseudorapidity in the range $1.2<|\eta|<2$ in the SCT endcaps. This is consistent with what can be observed from Fig. 23 but with the higher precision illustrating this more clearly. The efficiency for both the precision and fast tracking is again seen to be approximately constant with respect to changes in the $z_{0}$ position and pile-up interaction multiplicity.

The resolutions in the track pseudorapidity and inverse transverse momentum are shown in Fig. 29 as functions of the offline track pseudorapidity and transverse momentum themselves. The performance of the resolution at large trans- 


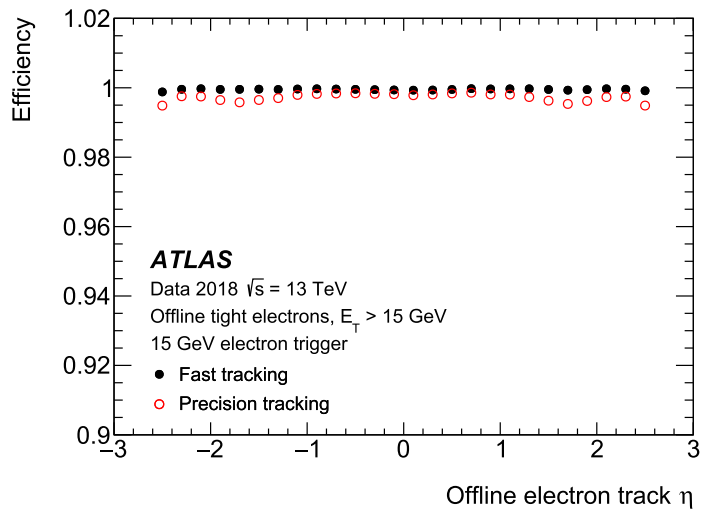

(a)

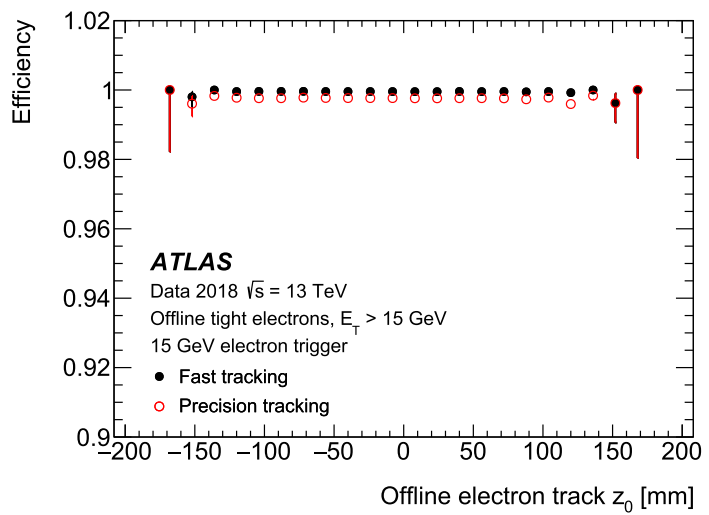

(b)

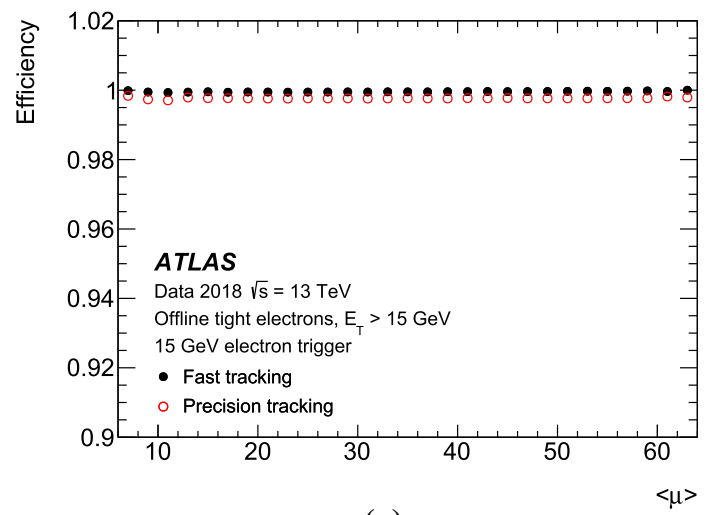

(c)

Fig. 28 The electron track finding efficiency versus a the offline electron track pseudorapidity, $\mathbf{b} z$ position at the beam line, and $\mathbf{c}$ the mean pile-up interaction multiplicity measured using the dilepton trigger

verse momentum is seen to be consistent with that observed in Fig. 24. The resolutions at lower transverse momentum, and versus pseudorapidity are also consistent, lying between the resolutions from the $10 \mathrm{GeV}$ and $26 \mathrm{GeV}$ triggers, but once again with a greatly reduced statistical uncertainty.

The worse resolution for the inverse of the transverse momentum for the precision tracking in comparison with the fast tracking is again seen at low transverse momenta and large pseudorapidities, as in Fig. 24. This can be understood as a consequence of the lower mean SCT hit multiplicity for the FTF tracks in the endcaps.

Figure 30 shows the mean SCT cluster multiplicity for the precision and fast tracks for the $E_{\mathrm{T}}>15 \mathrm{GeV}$ electron trigger tracks as functions of both transverse momentum and pseudorapidity of the matched offline electron candidate. The general variation of the multiplicity with pseudorapidity is the same as for the other signatures, but at large pseudorapidities, and for transverse momenta lower than the calorimeter cluster threshold, it is seen that the FTF tracks have a lower SCT hit multiplicity than the precision tracks.

Tracks from electron candidates which have radiated an energetic photon may have significant kinks such that their trajectory will not be helical. To account for this, the offline electron reconstruction runs a Gaussian sum filter [64] to allow for significant kinks in tracks due to bremsstrahlung, whereas the electron track reconstruction used in the trigger during Run 2 was running the standard track reconstruction. As a consequence of the pixel requirements, when fitting tracks including the full complement of correctly identified SCT hits, the precision track candidates containing kinks will be less helical. The transverse momentum reconstruction near the beam line for the fitted helical track will therefore be affected as the track fit is pulled away from the true trajectory to compensate for the position of the outer SCT hits and so produce a fit with lower transverse momentum. For the FTF tracks, if the outer SCT hits are rejected in the fit, as suggested by Fig. 30, then the early sections of the track closest to the beam line before any bremsstrahlung will be better reconstructed and so the $p_{\mathrm{T}}$ reconstruction of the track nearest the beam line will be somewhat better.

This can be seen in Fig. 31a which shows the residual in the $1 / p_{\mathrm{T}}$ distribution for the fast track finder and precision tracking with respect to the offline electron track candidate $p_{\mathrm{T}}$ for candidates with the offline track $p_{\mathrm{T}}$ around $10 \mathrm{GeV}$ $\left(7.4<p_{\mathrm{T}} \leq 13.6 \mathrm{GeV}\right)$. Here the fast track finder residual distribution can be seen to be somewhat narrower than that from the precision tracking, which has a large tail extending to larger $1 / p_{\mathrm{T}}$ values reconstructed in the trigger, i.e. to lower $p_{\mathrm{T}}$. The central peak for the fast track finder is shifted to somewhat lower values, whereas the peak in the precision tracking is closer to zero, but with the large tail, pulling the mean to higher values.

This can be seen more concisely in the Fig. 31b which shows the mean of the residual distribution for the fast tracking finder and precision tracking as a function of the offline electron candidate track $p_{\mathrm{T}}$. This clearly shows that for tracks with very low $p_{\mathrm{T}}$ relative to the minimum electron $E_{\mathrm{T}}$, the offset of the mean is large and positive for the precision tracking, meaning a bias towards lower track $p_{\mathrm{T}}$ than that measured offline. For the fast track finder, the bias - predominantly from the small shift in the peak position - is towards 


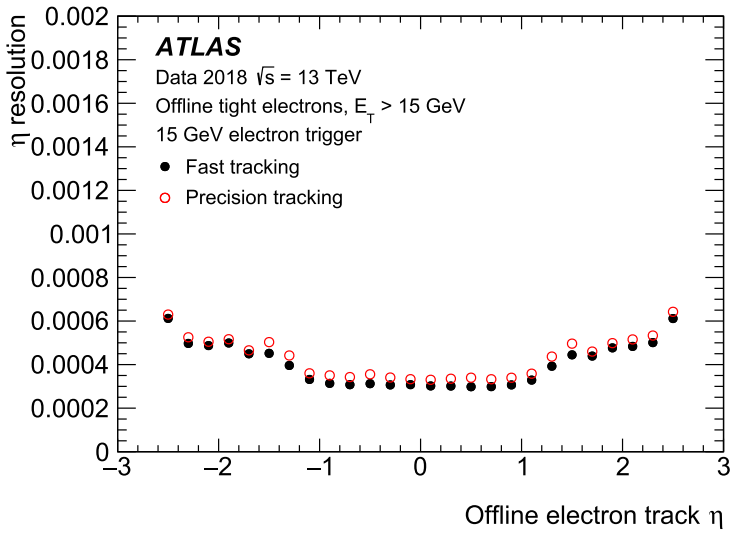

(a)

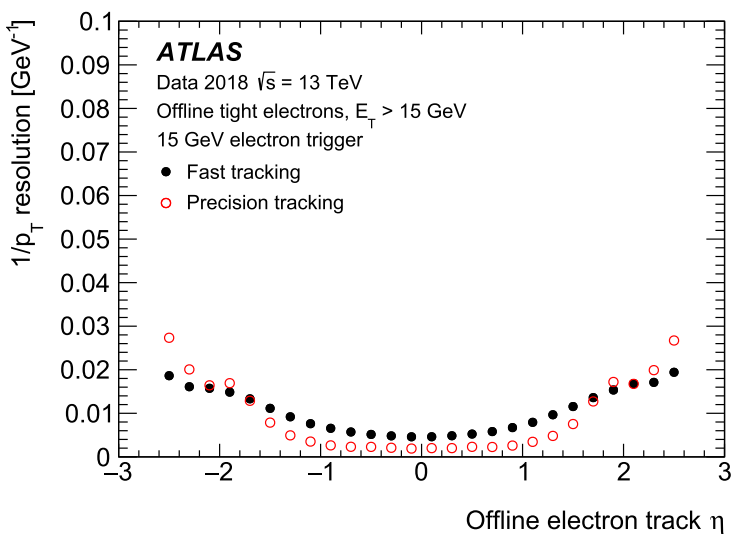

(c)

Fig. 29 The resolution in the pseudorapidity $(\mathbf{a}, \mathbf{b})$, and the inverse transverse momentum $(\mathbf{c}, \mathbf{d})$ as a function of offline track pseudorapidity and transverse momentum. The resolutions are shown for electrons

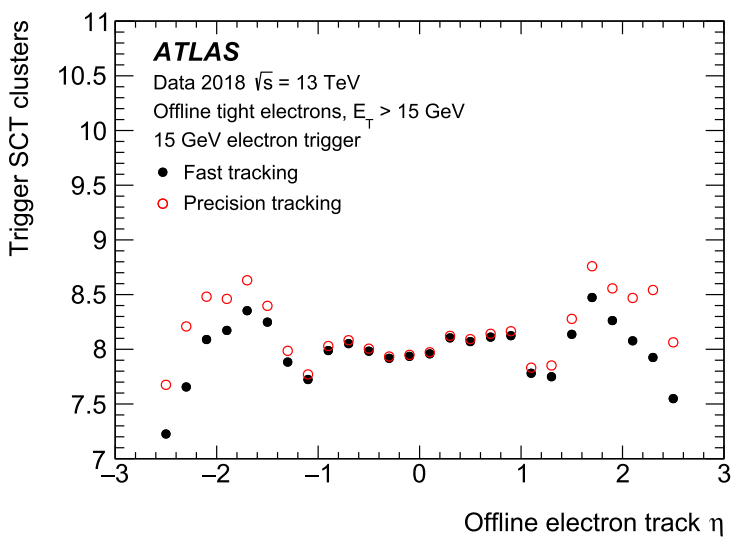

(a)

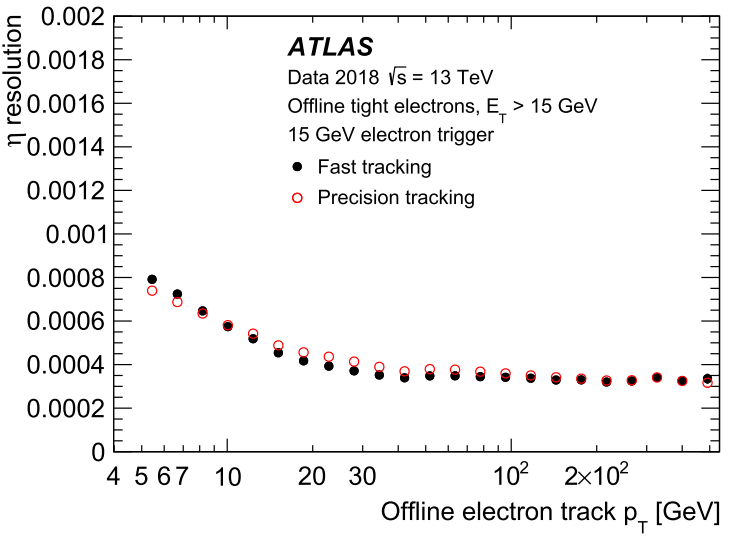

(b)

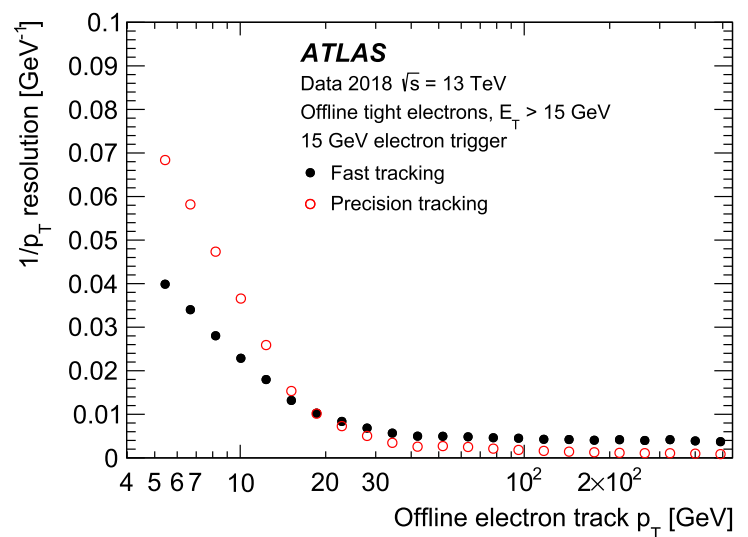

(d)

selected by the $E_{\mathrm{T}}>15 \mathrm{GeV}$ electron requirement from the dilepton support triggers, for both the fast tracking and precision tracking algorithms

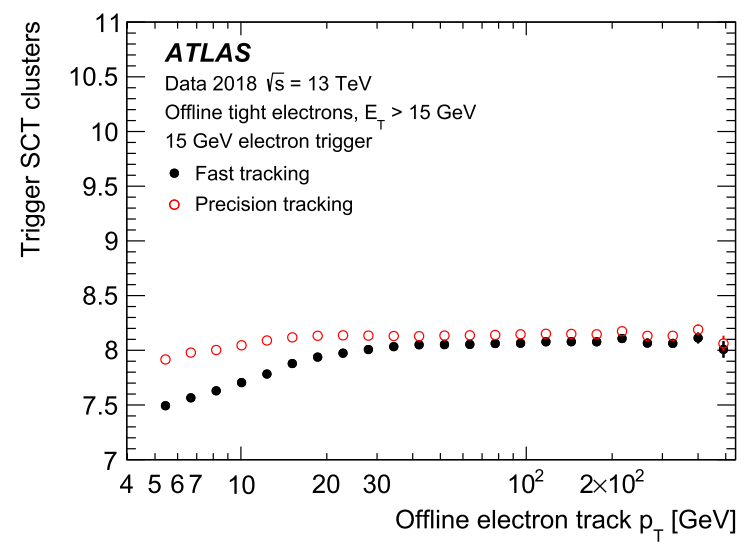

(b)

Fig. 30 The SCT cluster multiplicity for the trigger tracks matched to offline electron candidate tracks as a function of a the offline electron candidate pseudorapidity and $\mathbf{b}$ the offline electron candidate transverse momentum from the dilepton performance trigger

higher $p_{\mathrm{T}}$ than for the offline track, and so closer to the transverse energy of the electron candidate as determined by the cluster measured in the calorimeter.
For future data taking it may be possible for the trigger to include aspects of the Gaussian sum filter tracking, used 


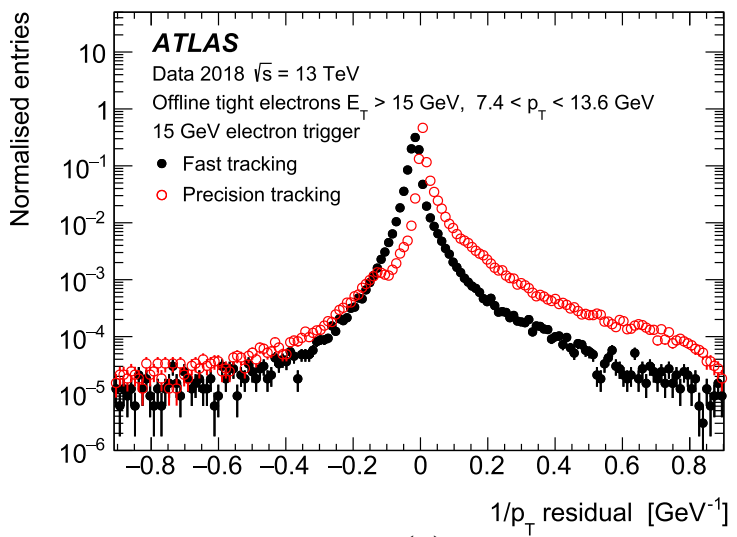

(a)

Fig. 31 The ID trigger residual in $1 / p_{\mathrm{T}}$ with respect to offline for the fast and precision tracking for electron candidates with the offline track transverse momentum the range in $7.4<p_{\mathrm{T}}<13.6 \mathrm{GeV}$ selected by

offline, to improve the transverse momentum resolution of the precision tracking.

\subsection{Tracking in the tau signature}

Figure 32 shows the efficiency of the tau two-stage tracking with respect to the offline tracking for offline tracks with $p_{\mathrm{T}}>1 \mathrm{GeV}$ originating from decays of offline tau lepton candidates $[8,13]$ with $p_{\mathrm{T}}>25 \mathrm{GeV}$. The multistage tracking used in the tau trigger signature [4,7] is described in detail in Sect. 3.4, but it is worth recalling that the first stage runs in a narrow RoI in $\eta-\phi$. The narrow $\phi$ region means that tracks at low $p_{\mathrm{T}}$ (up to around $5 \mathrm{GeV}$ ) may bend significantly in the solenoid field, and if near the edge of the RoI, may not be fully contained in the RoI and so could not the three years be reconstructed. This is partly taken into account when selecting reference tracks for the measurement of the efficiency for the different stages, where offline tau candidate tracks which are not fully contained within the RoI are rejected. The width of the RoI determines the lowest transverse momentum that can be reconstructed in an RoI. However, since the RoI originates from $(0,0)$ in the detector rather than at the beam line, some lower momentum tracks near the edges of an RoI in $\phi$ may still be subject to a reduction in efficiency if part of the track falls outside the RoI. Figure 32 shows the efficiencies for the fast tracking from the first and second stages together with that from the second stage precision tracking. Because of the RoI containment, the sample of tracks near threshold is quite small, particularly for the first stage tracking, resulting in a lower efficiency and a significantly larger uncertainty.

The track resolutions for $\eta, d_{0}$ and $z_{0}$ with respect to offline track $\eta$ and $p_{\mathrm{T}}$ are shown in Fig. 33. The precision tracking resolution is generally better than the fast tracking resolution. The resolution from the fast tracking in both the first

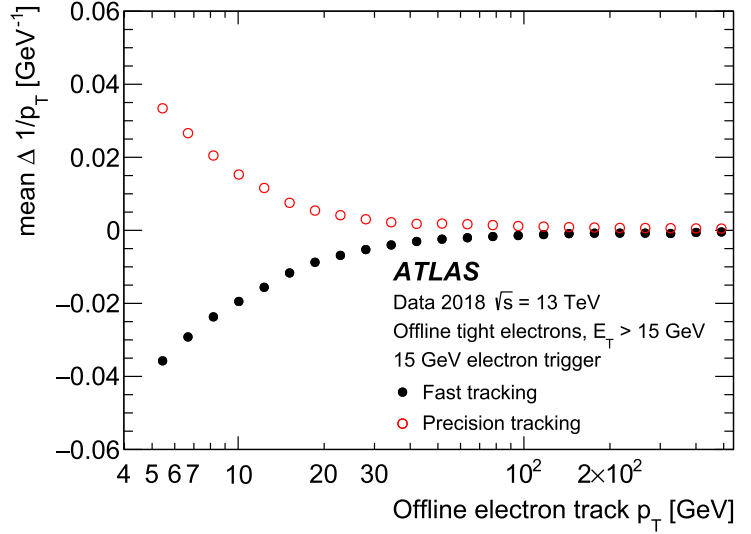

(b)

the $15 \mathrm{GeV}$ tag and probe trigger (a); and the mean of the residual as a function of the offline track $p_{\mathrm{T}}$ (b) for the fast and precision tracking

and second stages has essentially the same performance for offline tau track candidates with $p_{\mathrm{T}}>5 \mathrm{GeV}$ but differs for $p_{\mathrm{T}}<5 \mathrm{GeV}$. This is an artifact of the RoI containment requirement for the different RoI discussed above. It is worth noting that only the $d_{0}$ from the precision tracking reconstruction is used in the final selection of tau candidates online, for which the resolution with respect to offline is better than $20 \mu \mathrm{m}$ for tracks with $p_{\mathrm{T}}>5 \mathrm{GeV}$. When integrating over $p_{\mathrm{T}}$, the mean resolution lies in the range 15-20 $\mu \mathrm{m}$ for all pseudorapidities.

The effect of changes to the trigger between 2016, 2017, and 2018 can be seen for the second stage fast tracking in Fig. 34. The first stage tracking essentially underwent no changes between the three years, the differences being predominantly in the second stage processing. As seen in Fig. 34a, during 2016 a small inefficiency was observed at large pseudorapidities because of the tightening of the second stage RoI about the $z$-position of the leading track. The resolution of the predicted seed positions for seed pairs at large pseudorapidities becomes important. Approximations used in the layer positions for the seed finding were particularly affected by the worse resolution for seeds at large pseudorapidities, and as such the predicted position of the seed pair at the beam line was not precise enough to guarantee that the seed pairs fell within the selection window of the RoI, and consequently a significant fraction of seeds were being rejected. For 2017, the increased rate due to the higher pile-up occupancy required an additional change to the seed finding used for the FTF tracking, which resulted in a further deterioration of the efficiency at larger $|\eta|$, while the efficiency at central $\eta$ was unaffected.

Modifications to take account of the worse seed resolution at large pseudorapidities were under development, but were not ready for the start of data taking in 2017 . For 2018 


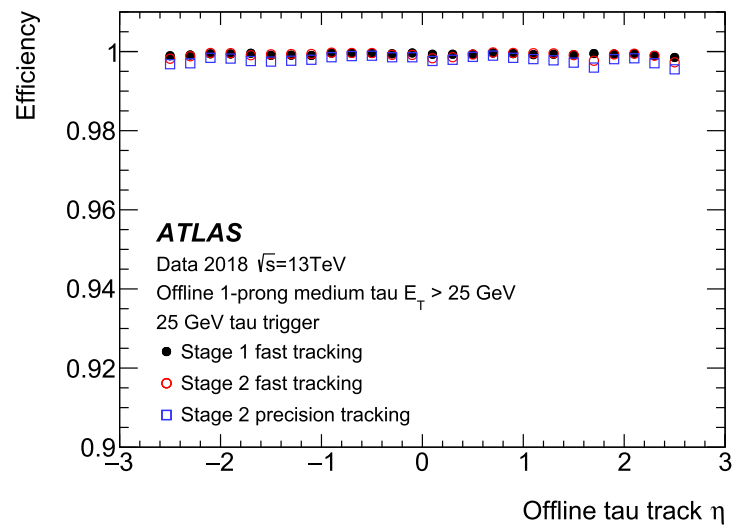

(a)

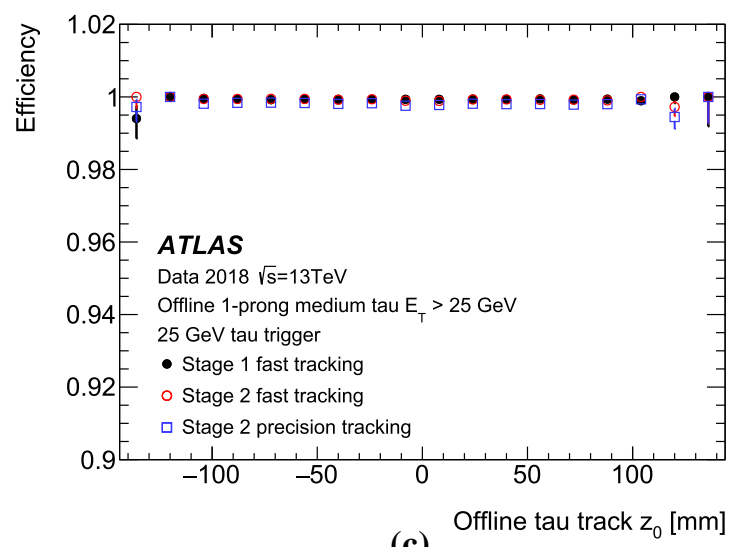

(c)

Fig. 32 The track finding efficiency of the ID trigger for tracks from tau decays with $p_{\mathrm{T}}>1 \mathrm{GeV}$. The efficiency is evaluated for the $25 \mathrm{GeV}$ tau performance trigger which does not use ID tracking information for the selection. The efficiency is shown as a function of $\mathbf{a}$ offline track $\eta$,

data taking, the seed finding was reimplemented to avoid the approximations and restore the efficiency at large $|\eta|$. The efficiency versus $p_{\mathrm{T}}$, seen in Fig. 34b, illustrates the significant loss of efficiency in 2017 at large $|\eta|$, which leads to an overall $3 \%$ loss of efficiency, improving to $2 \%$ at higher $p_{\mathrm{T}}$ where the tracks become more central. With the modifications to the seeding for 2018 running, the efficiency improves to better than $98 \%$ everywhere and better than $99.5 \%$ for $p_{\mathrm{T}}>1.2 \mathrm{GeV}$.

\subsection{Tracking in the $b$-jet signature}

As discussed in Sect. 3.4, for the $b$-jet trigger $[4,10]$, the tracking runs in two stages. The first stage runs the vertex tracking in narrow $\eta-\phi$ RoI about the jet direction for those jets with $p_{\mathrm{T}}>30 \mathrm{GeV}$, and which are aggregated into a super RoI before running the track reconstruction. The individual jet RoI about each jet are fully extended from $-225<z<225 \mathrm{~mm}$ along the beam line. These tracks

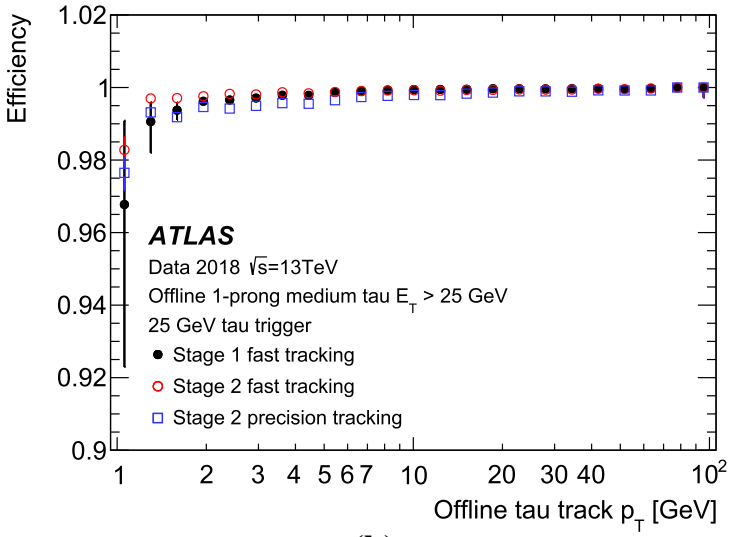

(b)

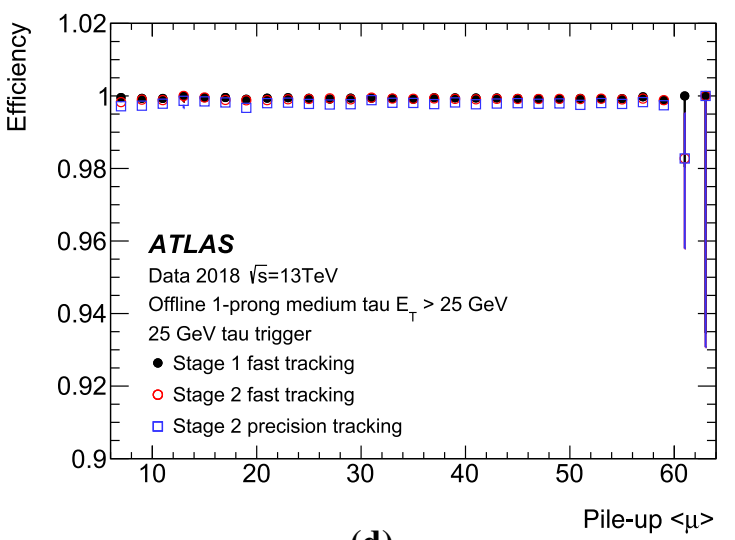

(d)

$\mathbf{b}$ offline track $p_{\mathrm{T}}, \mathbf{c}$ offline track $z_{0}$ and $\mathbf{d}$ mean number of interactions per bunch crossing. Statistical Bayesian uncertainties are shown, using a $68.3 \%$ confidence interval and uniform beta function prior

are then used to reconstruct the vertex for the second stage processing. From 2017, to reduce the processing time, the pattern recognition for the first stage tracking ran only for track candidates with $p_{\mathrm{T}}>5 \mathrm{GeV}$.

The purpose of the vertex tracking is primarily to identify sufficient tracks with a high enough transverse momentum to reconstruct the primary interaction vertex corresponding to the jets to refine the RoI for the tracking used for the $b$ tagging. Since the first stage vertex is used only to define the $z$ position of the second stage RoI, the vertex resolution is not required to be better than approximately $1 \mathrm{~mm}$. The size of the jet RoI used in the first stage, and the higher threshold for track finding were carefully tuned for 2017 data taking and chosen to keep the processing time to a manageable level in events with high jet multiplicities whilst still maintaining a high enough efficiency for the $b$-jet events of interest.

The second stage runs both the fast and the precision tracking in wider RoI centred on the jet axes, with $|\Delta \eta|<0.4$ and $|\Delta \phi|<0.4$ relative to the RoI direction, and with the 


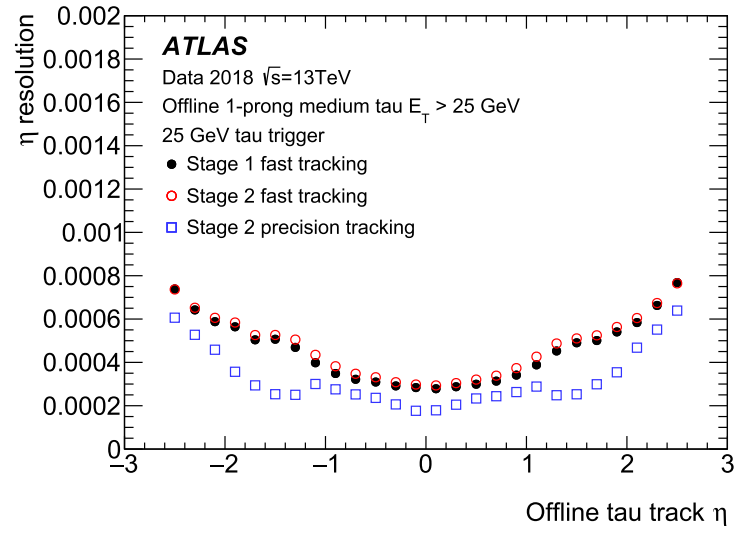

(a)

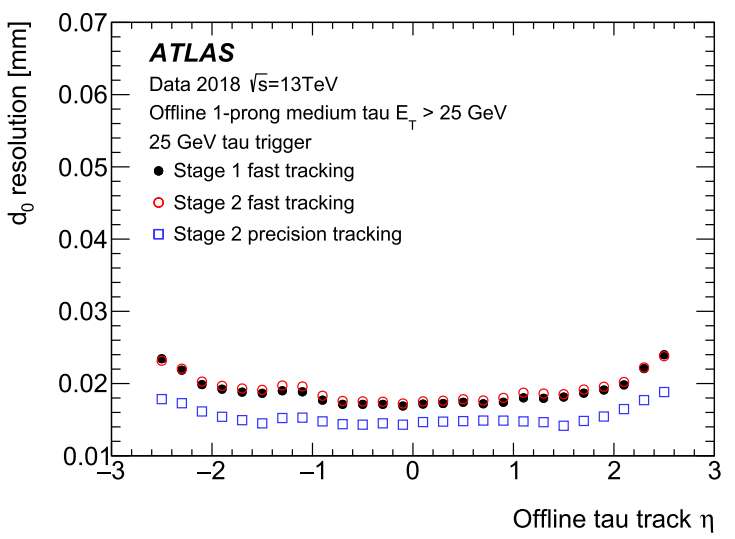

(c)

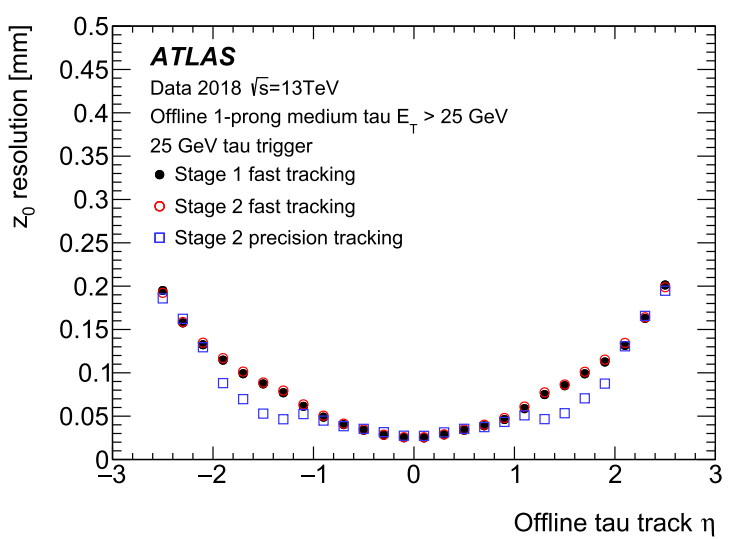

(e)

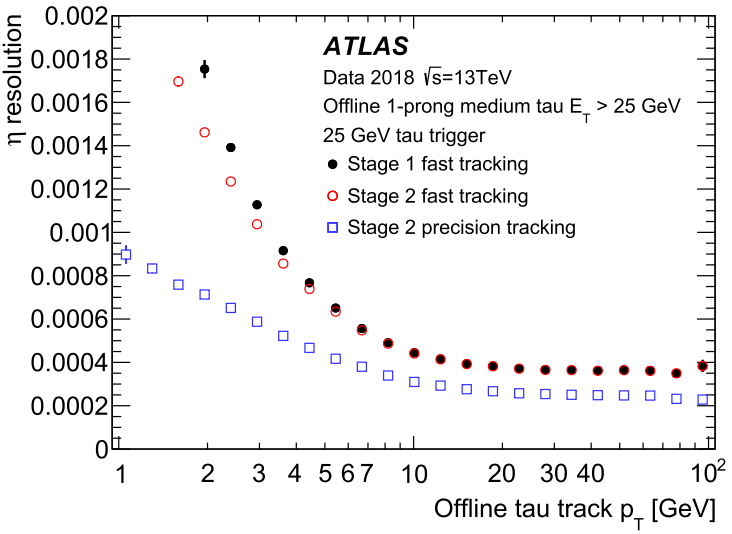

(b)

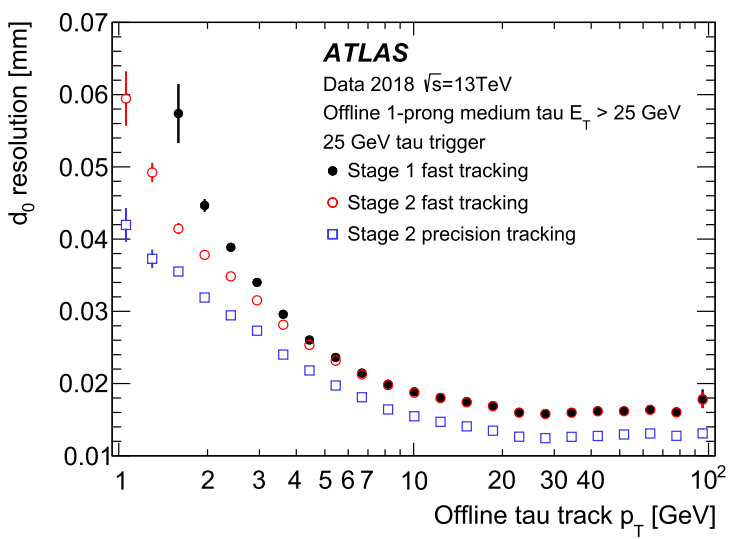

(d)

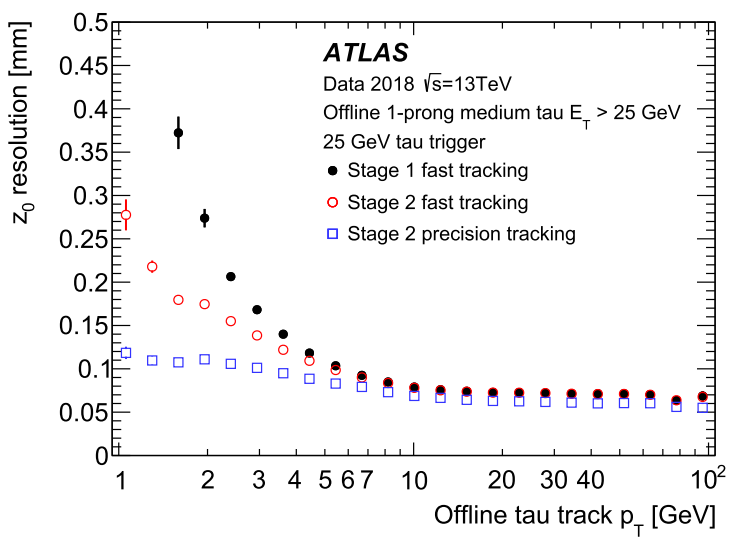

(f)

Fig. 33 The tau signature tracking resolution in $\mathbf{a}, \mathbf{b}$ pseudorapidity, $\mathbf{c}, \mathbf{d}$ transverse impact parameter, and $\mathbf{e}, \mathbf{f}$ track $z$ position along the beam line, all as functions of the matching offline track pseudorapidity and transverse momentum

centre of each RoI in $z$, at the position of the primary vertex identified in the first stage. These second stage RoI have a smaller extent, $\Delta z<10 \mathrm{~mm}$, about this $z$ position.

Only the tracks from the precision tracking of the second stage are used for the $b$-jet selection. The precision tracks are also used in the trigger for the global sequential jet energy calibration [65].
For the selection of the reference tracks used for the analysis of the efficiency and resolution of the $b$-jet signatures, a tight offline selection was used which takes into account the detector geometry and performance as a function of pseudorapidity. The selection criteria are: 


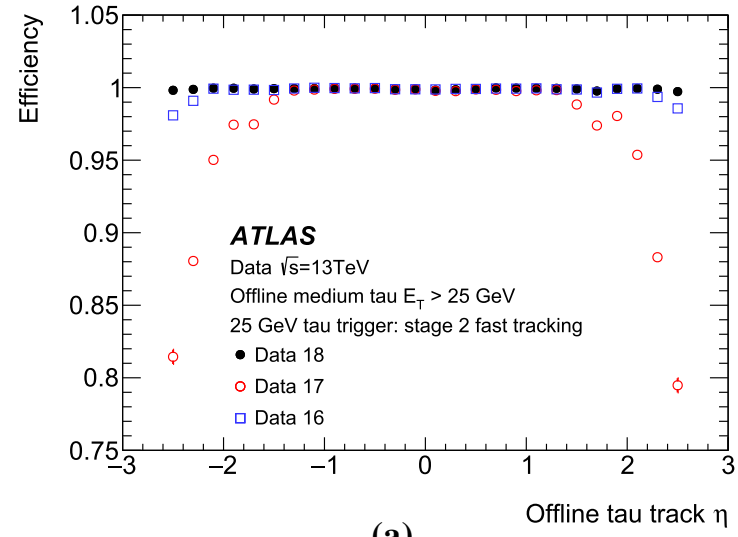

(a)

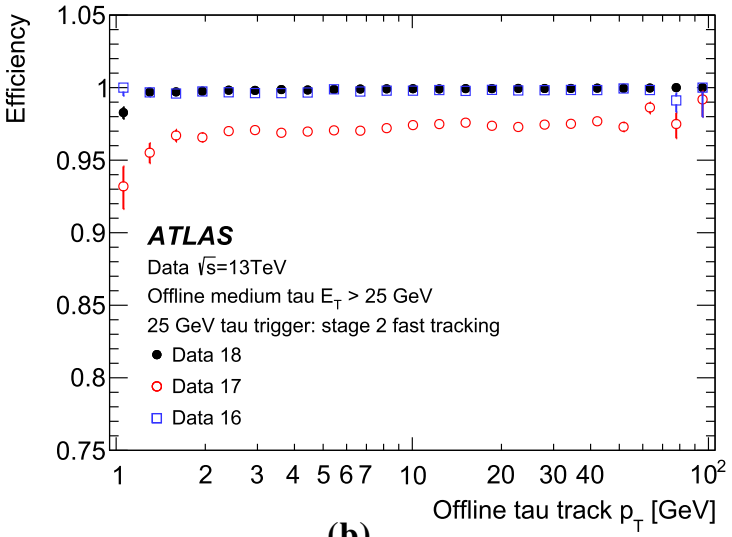

(b)

Fig. 34 The efficiency of the tau second stage fast tracking as a function of a pseudorapidity and $\mathbf{b}$ transverse momentum, comparing the performance in 2016, 2017, and 2018

- greater than eight silicon hits if $|\eta| \leq 1.65$, or eleven or more silicon hits if $|\eta|>1.65$,

- at least one hit in the IBL and next innermost pixel layer if passing through active modules in those layers,

- no missing pixel hits in any active modules in layers at a larger radius than the innermost pixel hit on a track,

- at least one pixel hit from any of the remaining layers, should hits not be present in the IBL and next innermost pixel layer.

The efficiency is measured using dedicated triggers which run as standard $b$-jet physics signatures, but without selecting on the tracking information or running the $b$-tagging selection.

The efficiency for the tracking from both stages in the $b$-jet signature is shown in Fig. 35 for the $55 \mathrm{GeV}$ threshold $b$-jet trigger, shown as a function of the offline track parameters and $\langle\mu\rangle$. Figure 36 compares the precision and fast tracking efficiencies for the jet tracking from the $55 \mathrm{GeV}$ and $150 \mathrm{GeV}$ threshold triggers. By design, the first-stage vertex tracking only reconstructs tracks with $p_{\mathrm{T}}>5 \mathrm{GeV}$ and thus only offline tracks with $p_{\mathrm{T}}>5 \mathrm{GeV}$ were selected for the vertex tracking reference sample used for Fig. 35. For offline tracks above approximately $1.2 \mathrm{GeV}$ the second stage fast tracking efficiency is better than $99.5 \%$. For tracks near $1 \mathrm{GeV}$ the fast tracking efficiency is better than $98 \%$ but the precision tracking efficiency is lower - approximately $85 \%$. $^{4}$ This is expected and is a consequence of an explicit $1 \mathrm{GeV}$ cut placed on the tracks from the precision tracking in the $b$-jet signature implemented for processing-latency reasons. As such the reconstruction for tracks near the threshold for

\footnotetext{
$\overline{4}$ This is not shown in this figure, since the required axis range would render other more relevant features in the figure more difficult to discern, but can be seen in Fig. 39.
}

which the trigger $p_{\mathrm{T}}$ would be reconstructed to be lower than the $1 \mathrm{GeV} p_{\mathrm{T}}$ threshold is terminated early.

For offline tracks with transverse momenta greater than approximately $10 \mathrm{GeV}$ the precision tracking efficiency starts to fall slightly, and is more significant for the higher threshold $b$-jet triggers. This is largely a consequence of the reduced pixel hit multiplicity found on the precision tracks for the higher threshold $b$-jet triggers. Although the hits are largely present on the tracks from the fast tracking, the tighter hit selection cuts on the precision tracks used to improve the purity relative to the fast tracking leads to this reduction. The effect of this can be seen in Fig. 37, which compares the multiplicity of trigger tracks with that of offline tracks contained within the corresponding RoI and associated with the primary vertex. The second stage precision and fast tracking must of course have an identical set of offline tracks contained within their common RoI, whereas the multiplicity of the second stage fast tracks themselves is significantly higher than the precision tracking multiplicity, which is, in turn, much closer to that for the offline tracks. For this study, it should be noted that the selection applied to the precision tracks is looser than for the offline tracks.

However, if using the same selection as used for the reference offline tracks for the determination of the efficiency, but applying instead to the tracks from the precision tracking, the purity of the $b$-jet precision tracks with respect to the offline tracking is seen to be better than $99 \% .^{5}$ In the trigger, additional track selection can be applied by any subsequent algorithm within the trigger which is a client of the tracking, to further improve the track purity. As such, any more detailed analysis of the track purity would be very depen-

\footnotetext{
5 Here, the purity is defined as the fraction of the trigger tracks that have a matching offline track when applying the same matching criteria used for determination of the efficiency.
} 


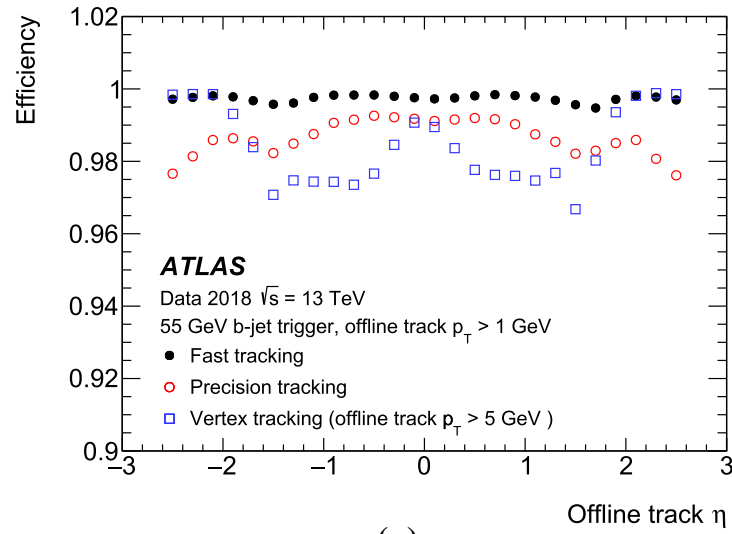

(a)

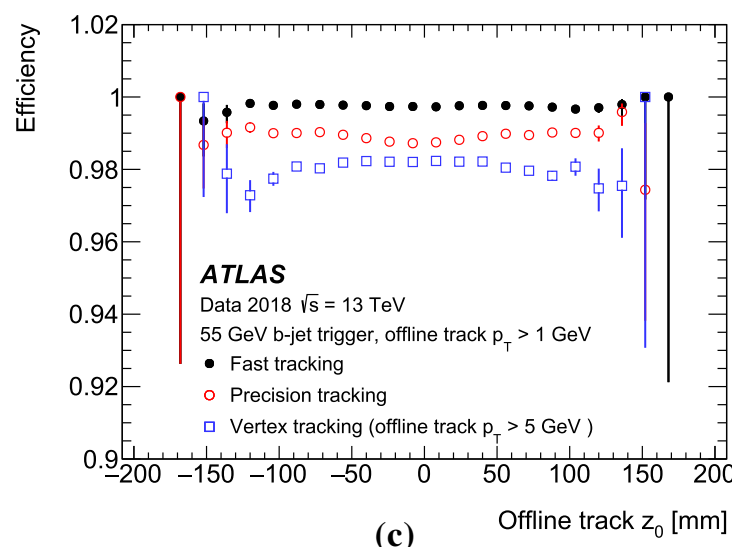

(c)

Fig. 35 The performance of the tracking in the $b$-jet signature, comparing the RoI based jet tracking with vertex tracking. Shown are the efficiencies with respect to $\mathbf{a}$ the pseudorapidity, $\mathbf{b}$ transverse momentum, and $\mathbf{c}$ the $z_{0}$ position at the point of closest approach to the beam for

dent on the specific requirements of that selection and is thus beyond the scope of the current discussion.

The tighter hit selection in the precision track reconstruction and the significantly lower efficiency near $1 \mathrm{GeV}$ are largely responsible for the lower overall efficiency of the precision tracking relative to the fast tracking. There is a clear structure in pseudorapidity - the efficiency being slightly lower in the endcaps and for central $z$. The small fall in efficiency with increasing $\langle\mu\rangle$ is more significant for the precision tracking, again driven largely by the lower efficiency at low $p_{\mathrm{T}}$.

Figure 38 shows the track resolutions for the fast and precision tracking from the second stage of the $b$-jet trigger for the $55 \mathrm{GeV}$ and $150 \mathrm{GeV}$ signatures. Shown are the resolutions for the pseudorapidity, transverse impact parameter and track $z$-vertex position, as functions of the offline track pseudorapidity and transverse momentum. The resolutions are estimated using data from the full 2018 data set, and so include small run-by-run variations in the residual distributions.

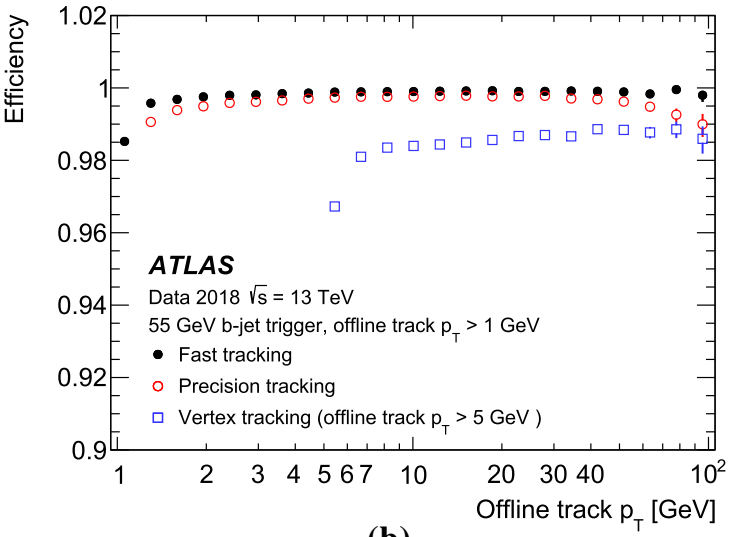

(b)

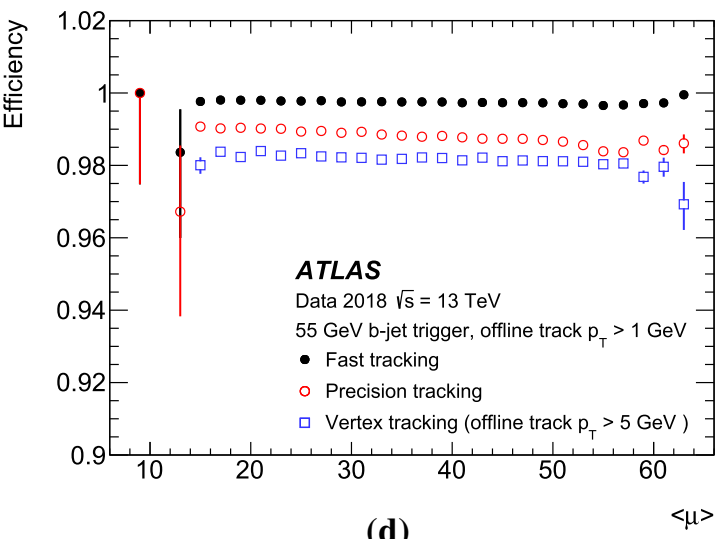

the offline reference tracks, and $\mathbf{d}$ with respect to the mean pile-up interaction multiplicity. Statistical Bayesian uncertainties are shown, using a $68.3 \%$ confidence interval and uniform beta function prior

The precision tracking provides significantly better resolution for all variables at lower track $p_{\mathrm{T}}$. However, there appears to be a slight deterioration of the $d_{0}$ resolution with increasing $p_{\mathrm{T}}$ for the high $E_{\mathrm{T}}$ jet trigger. This is correlated with a slight loss of pixel hits and corresponding loss in efficiency for the precision tracking at higher $p_{\mathrm{T}}$ in the high $E_{\mathrm{T}}$ triggers from Fig. 36.

Finally, in discussing the performance of the $b$-jet trigger one should consider the changes to the trigger during Run 2. In 2017, to deal with the increasing pile-up interaction multiplicity, the size of the first stage RoI in $\eta-\phi$ about each jet in the super RoI was reduced from $0.4 \times 0.4$ to the $0.2 \times 0.2$ size discussed in Sect. 3.4. The $z$ extent along the beam line remained unchanged. In addition the minimum track $p_{\mathrm{T}}$ used in the pattern recognition for the reconstruction of the vertex tracks was increased to $5 \mathrm{GeV}$. As in the case of the second stage tracking in the tau signature discussed in Sect. 5.5 the track finding in the second stage fast tracking was affected by the seeding. In the case of the $b$-jet trigger, the second stage RoI $z$-width was 


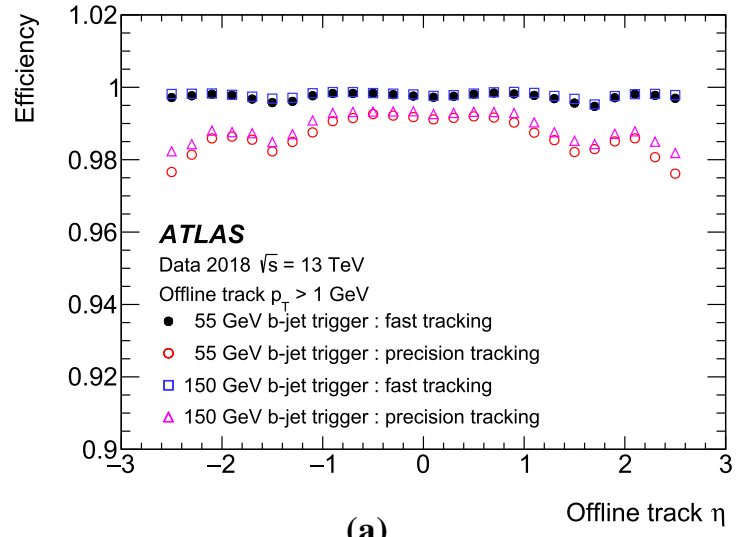

(a)

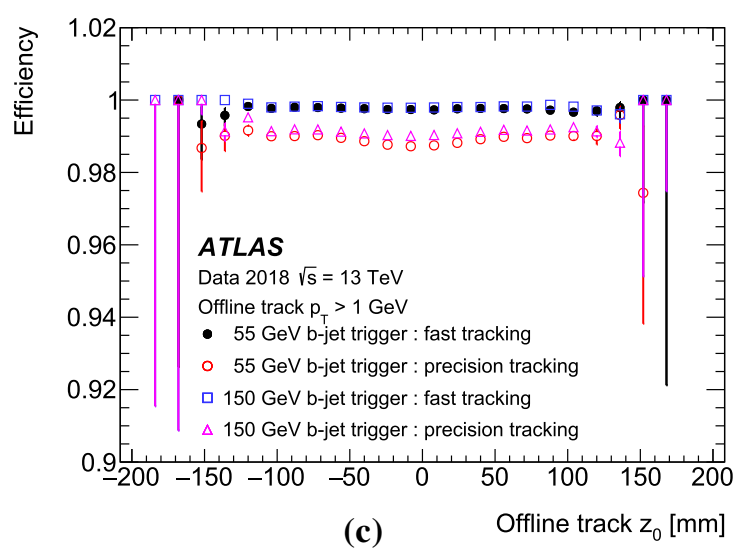

Fig. 36 The $b$-jet trigger tracking efficiency for tracks with $p_{\mathrm{T}}>$ $1 \mathrm{GeV}$ within jets shown as a function of $\mathbf{a}$ offline track $\eta$, $\mathbf{b}$ offline track $p_{\mathrm{T}}, \mathbf{c}$ offline track $z_{0}$, d the mean number of interactions per bunch crossing. The efficiency is evaluated for the $55 \mathrm{GeV} b$-jet trigger

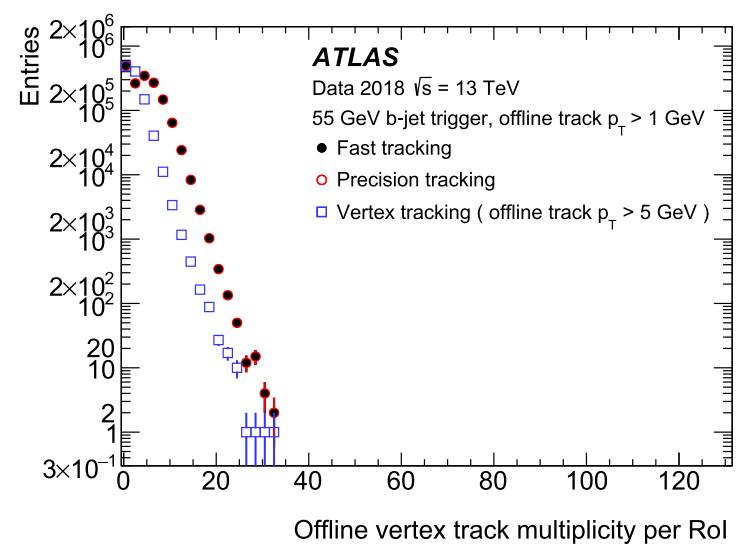

(a)

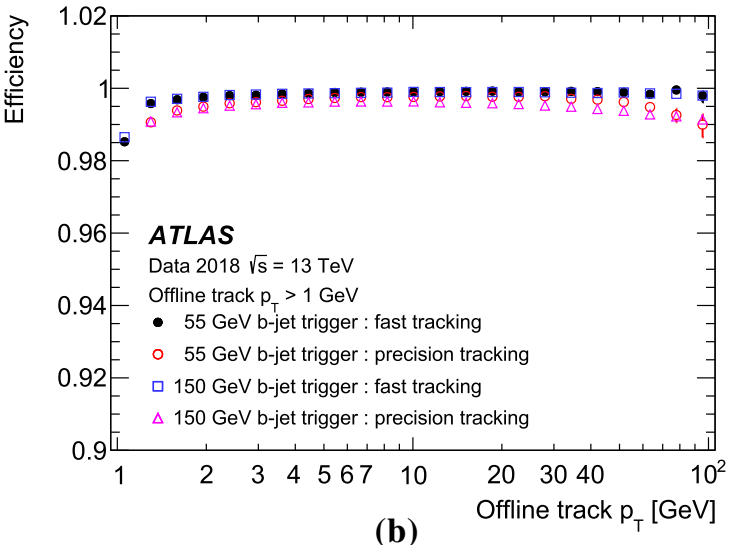

(b)

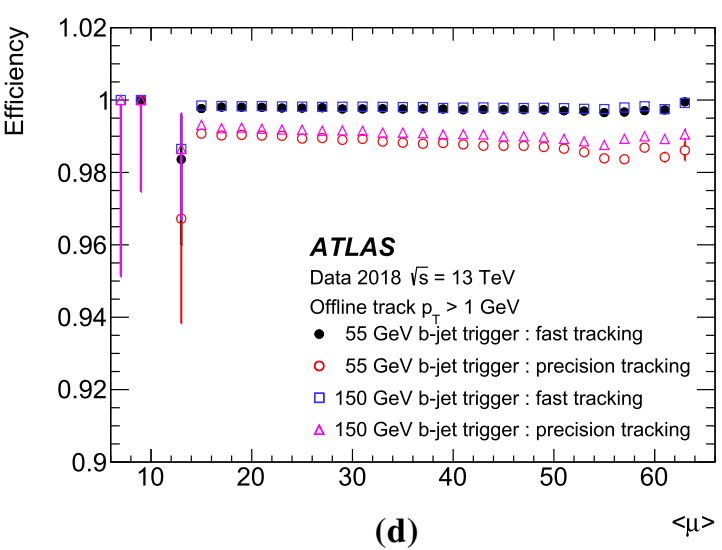

and is shown for the fast and precision tracking in both the vertex finding, and jet tracking stages of the $b$-jet ID trigger. Statistical Bayesian uncertainties are shown, using a $68.3 \%$ confidence interval and uniform beta function prior

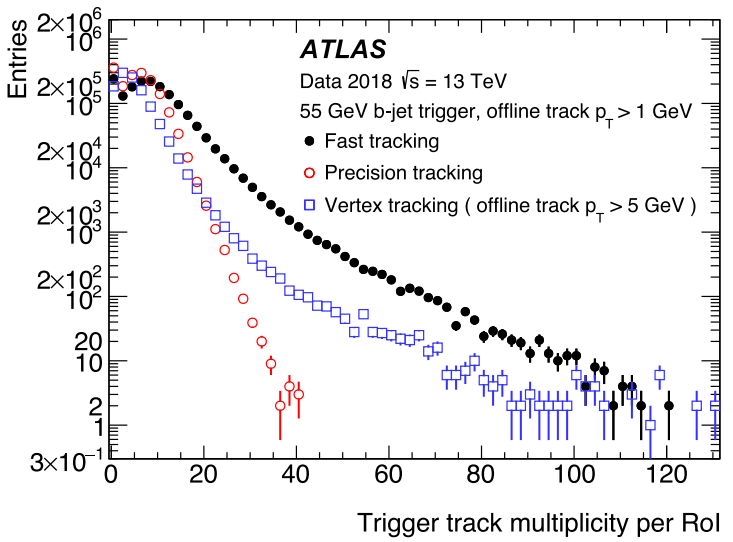

(b)

Fig. 37 The multiplicity of tracks found by the offline reconstruction, associated with the primary vertex and contained within the respective $b$-jet RoI (a), and the multiplicity of trigger tracks per RoI (b)

larger than for the tau signature. The resulting reduction in the efficiency of the fast tracking in the second stage jet RoI can be seen in Fig. 39 but this reduction is not as signifi- cant as for the tau signature. In contrast to the tau case, this loss in efficiency is seen to lie almost exclusively at lower $p_{\mathrm{T}}$ where the efficiency in 2017 is approximately $1 \%$ lower 


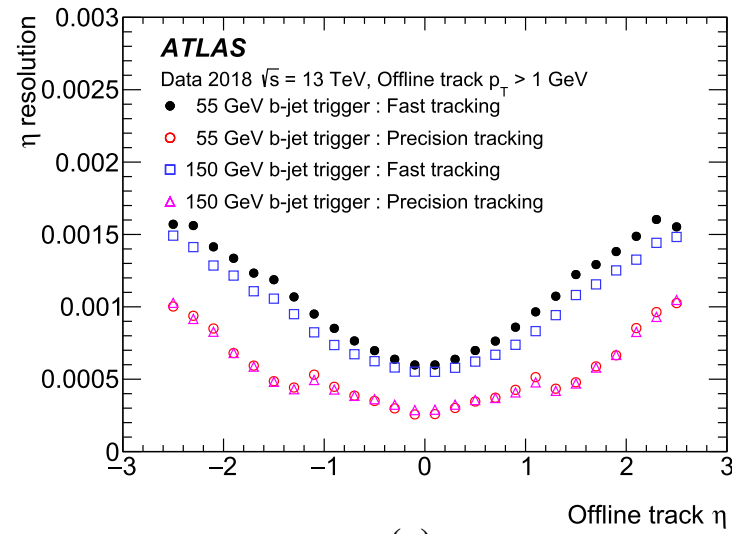

(a)

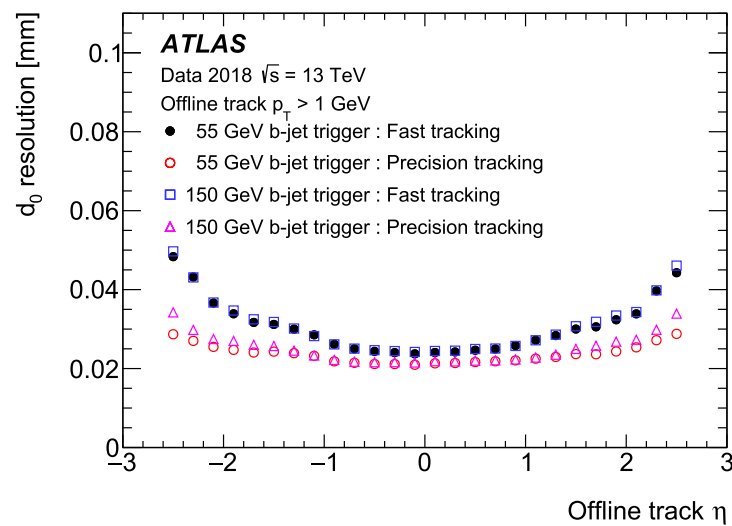

(c)

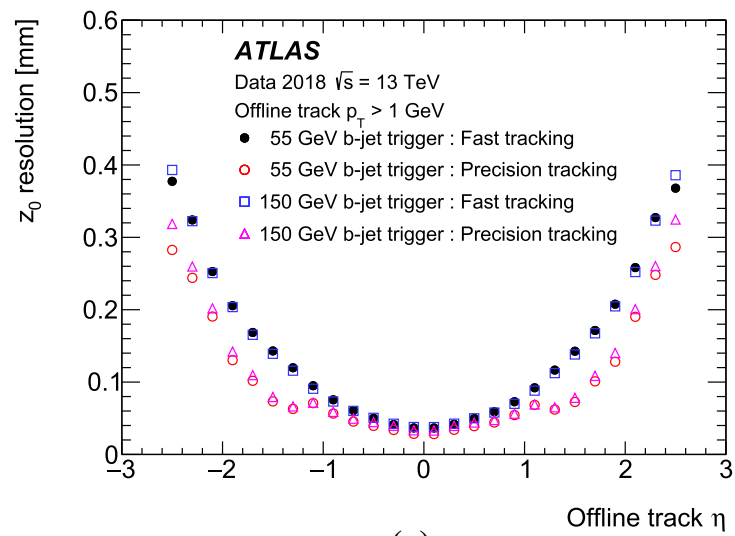

(e)

Fig. 38 The track resolutions for the fast and precision tracking from the second stage of the $b$-jet trigger for the $55 \mathrm{GeV}$ and $150 \mathrm{GeV}$ signatures. Shown are the resolutions for the trigger track pseudorapidity

than in either 2016 or 2018 . For 2018, the fixes to the seeding allowed the efficiency of the fast tracking in the second stage RoI to increase, but not quite recover to the level of 2016, where the LHC operated with significantly lower pileupmultiplicity.

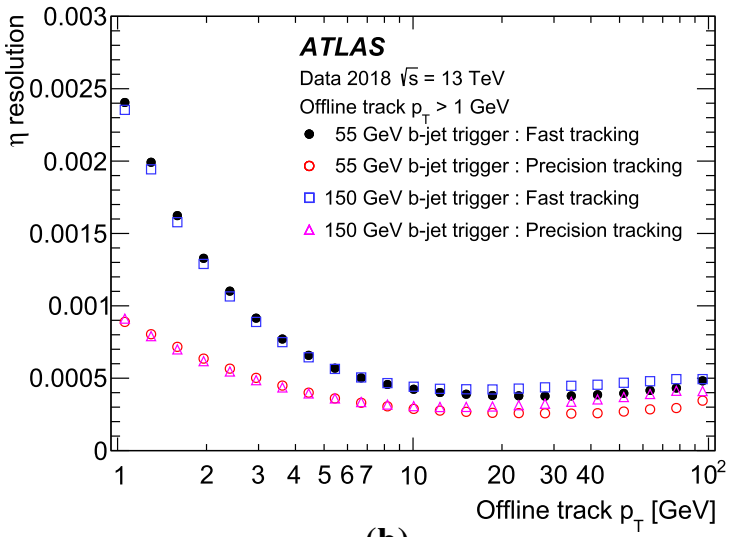

(b)

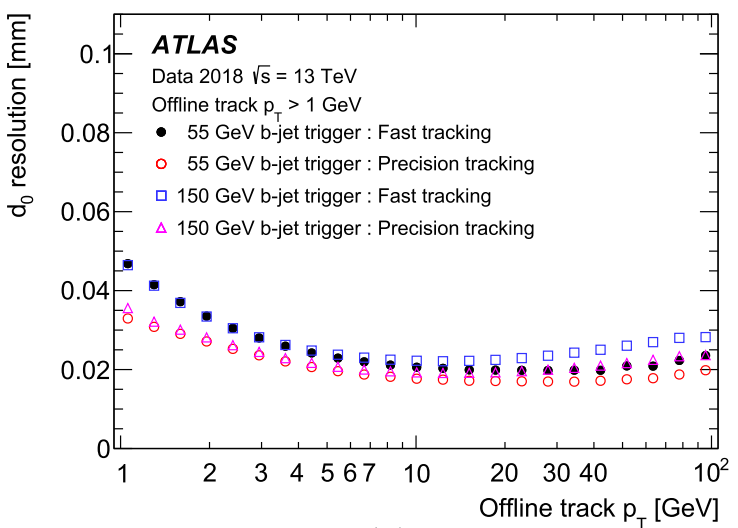

(d)

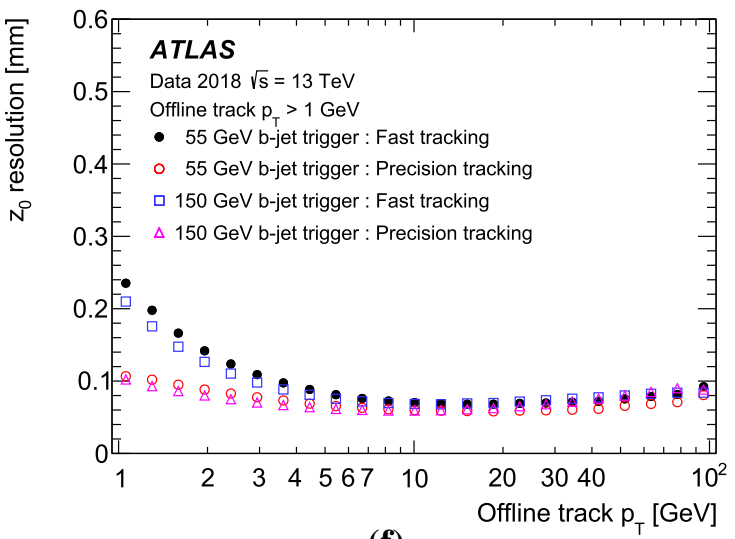

(f)

$(\mathbf{a}, \mathbf{b})$, transverse impact parameter $(\mathbf{c}, \mathbf{d})$ and track $z$ position $(\mathbf{e}, \mathbf{f})$, as functions of the offline tracks pseudorapidity and transverse momentum

\subsection{Vertex finding performance}

During Run 2, the $b$-jet vertex tracking stage ran with both vertex algorithms discussed in Sect. 3.3.4. For the online vertex selection, the more robust, but less precise histogramming vertex algorithm was used to identify vertex candidates for 


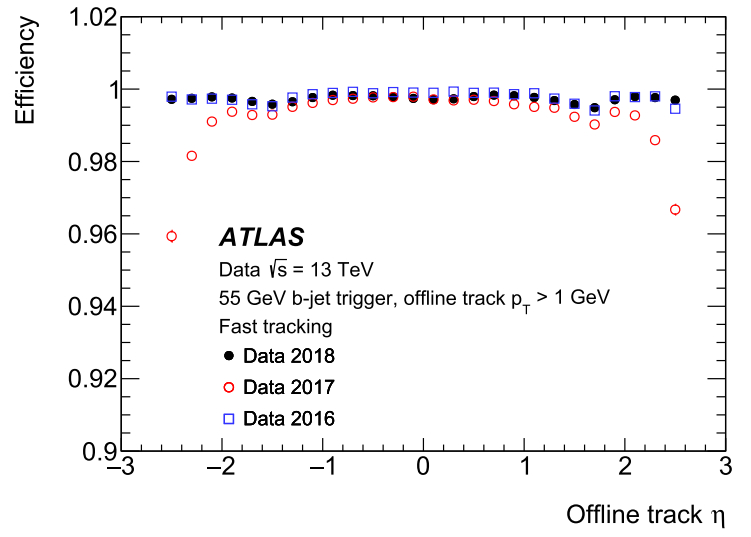

(a)

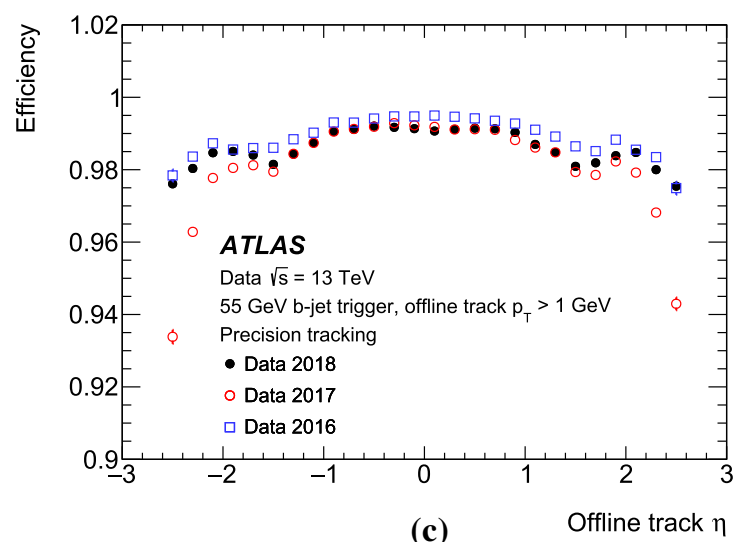

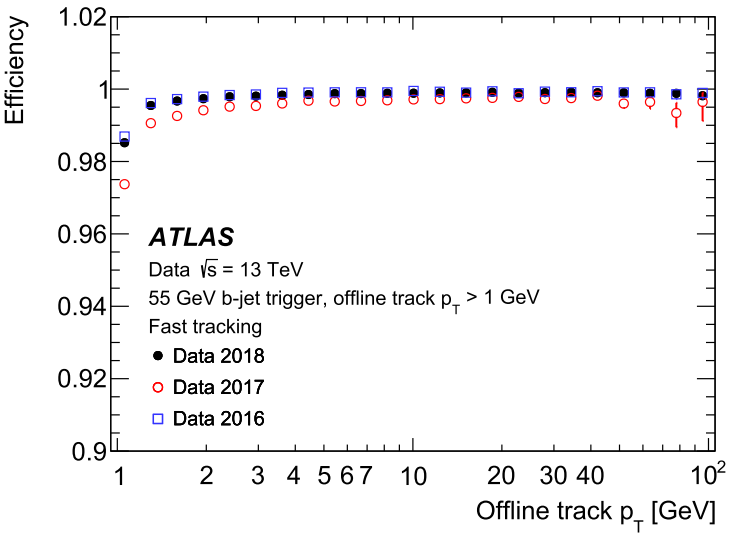

(b)

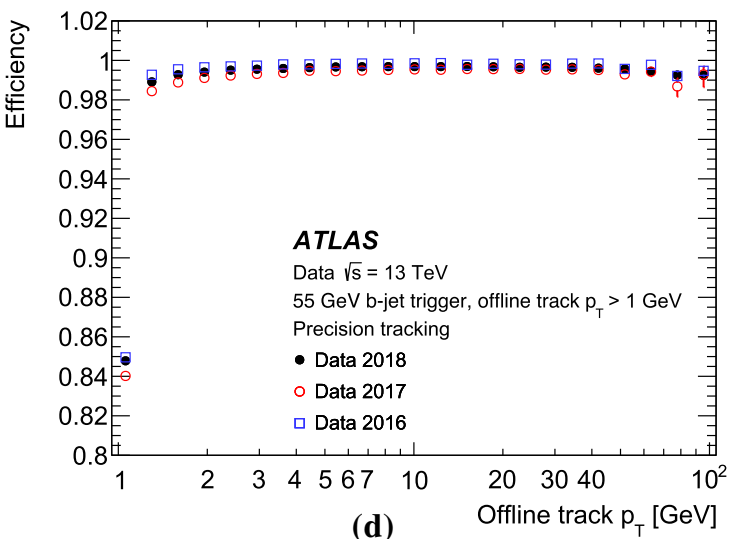

(d)

Fig. 39 The $b$-jet second stage tracking performance efficiency differences between 2016, 2017, and 2018

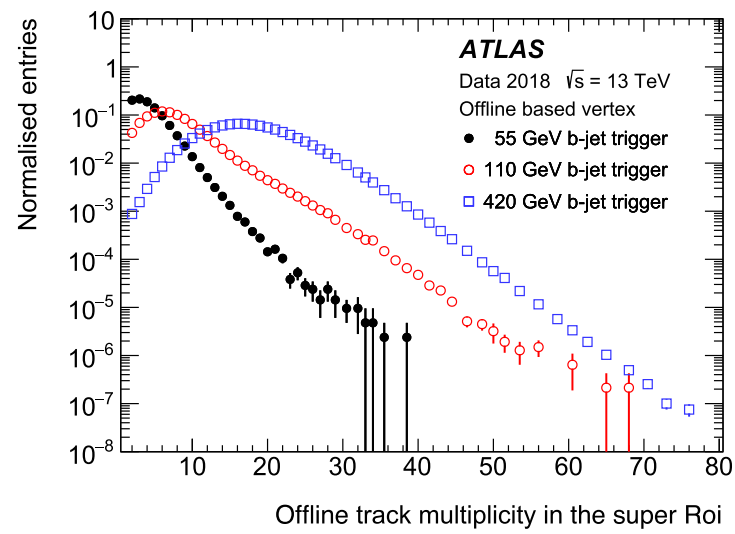

(a)

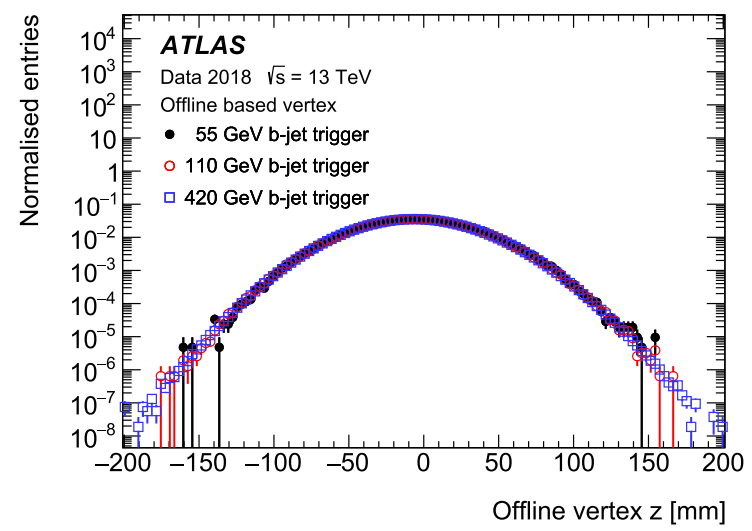

(b)

Fig. 40 The distribution of the offline track multiplicity for all selected offline tracks within the super RoI used for the vertex tracking (a), and their distribution in track $z_{0}$ position at the beam line (b)

those events where the offline based vertex algorithm failed to find a vertex. For the measurement of the performance, the online vertex efficiency is calculated for the single offline vertex candidate with the highest sum of the squared transverse momenta from the bunch crossing.
Since the offline vertex algorithm [66] runs over all offline tracks in the event, the multiplicity of offline tracks associated with the vertex can be quite high. In contrast, as the trigger runs only on those tracks in the jet super RoI, the multiplicity of tracks to be considered is much lower. As the offline vertex was intended to be used in a high occupancy environment, 


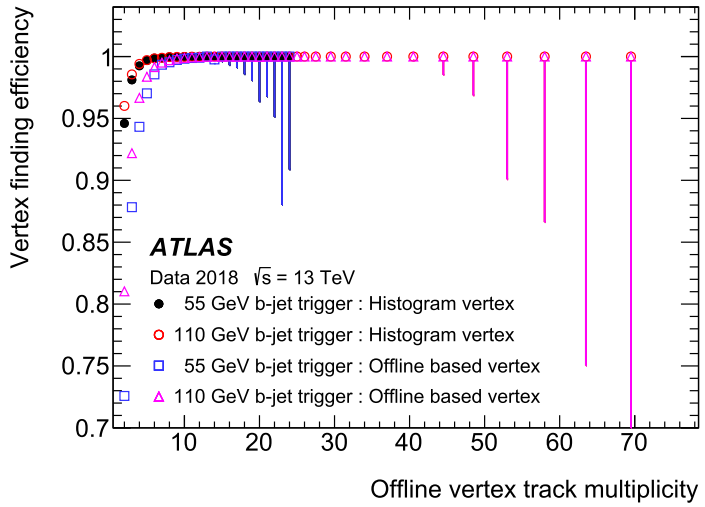

(a)

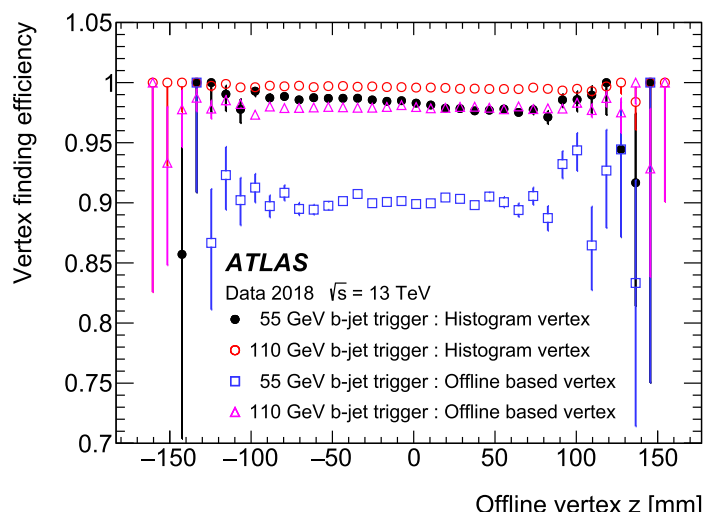

(b)

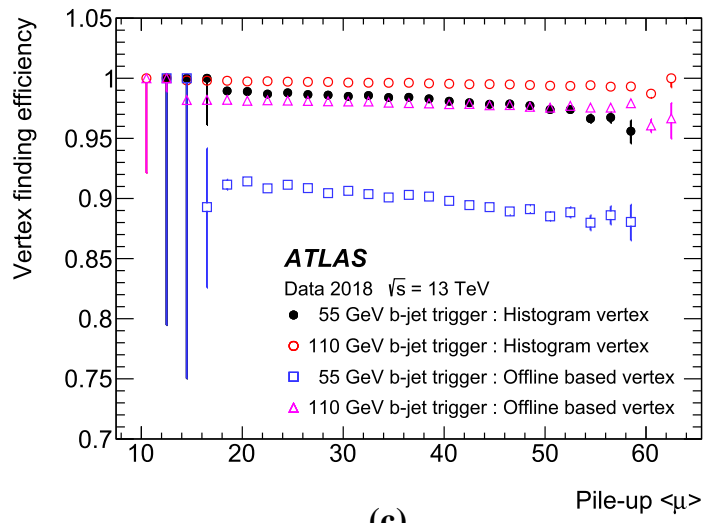

(c)

Fig. 41 The vertex algorithm efficiencies for low and intermediate $E_{\mathrm{T}}$ $b$-jet triggers. Shown are the efficiencies a versus the offline track multiplicity for tracks in the super RoI; $\mathbf{b}$ versus the $z_{0}$ position of the offline vertex; and c versus the mean pile-up interaction multiplicity. Statistical Bayesian uncertainties are shown, using a $68.3 \%$ confidence interval and uniform beta function prior

the performance in lower multiplicity conditions, may not be expected to be optimal.

In order to characterise the vertex in terms of track multiplicity, only those offline tracks associated with the vertex and which lie within the super RoI are counted. It is possible that the primary vertex identified by the offline reconstruc- tion does not in fact have any tracks on the vertex that lie within the super RoI pointing to the jets. It is observed that this possibility increases with the pile-up multiplicity since the chance of a pile-up vertex with a high enough track multiplicity to be chosen as the primary vertex in preference to the vertex from which the jets originate increases with the number of separate interactions in the event. Since, in this case, the offline algorithm used to select the offline primary vertex would in fact have selected a pile-up vertex candidate rather than the hard interaction corresponding to the jets, such events are excluded from the study.

As an additional selection, only those events in which there are at least two trigger jets in the super RoI within the tracking pseudorapidity acceptance $(|\eta|<2.5)$ and with $E_{\mathrm{T}}>30 \mathrm{GeV}$ are considered. The $E_{\mathrm{T}}$ requirement is a consequence of the super RoI, which is created only for trigger jets with $E_{\mathrm{T}}$ above $30 \mathrm{GeV}$.

Figure 40 shows the distribution of the $z$ position of the offline primary vertex candidates together with the multiplicity of offline tracks within the super RoI with $p_{\mathrm{T}}>5 \mathrm{GeV}$ for three $b$-jet triggers with $E_{\mathrm{T}}$ thresholds of $55 \mathrm{GeV}, 110 \mathrm{GeV}$ and $420 \mathrm{GeV}$. The multiplicity is for all selected offline tracks in the super RoI, not simply those associated with the offline primary vertex. For the higher $E_{\mathrm{T}}$ triggers, the track multiplicities are significantly higher as expected. Although not shown here, the mean $p_{\mathrm{T}}$ of the tracks within the jets is also harder for the higher $E_{\mathrm{T}}$ triggers. The distribution of the track $z$-positions is approximately Gaussian, and is largely independent of the jet threshold, again as expected.

The efficiency for identifying the vertex candidates in the trigger is shown in Fig. 41 for the $55 \mathrm{GeV}$ and $110 \mathrm{GeV}$ triggers, as a function of the offline track multiplicity in the super RoI, the $z$ position of the offline vertex, and the mean offline pile-up interaction multiplicity of the event. Figure 42 compares the efficiencies for the $110 \mathrm{GeV}$ and $420 \mathrm{GeV}$ triggers.

Shown in Fig. 41a, the vertex finding efficiency rises steeply with track multiplicity and reaches full efficiency for the histogram based algorithm for events with more than four vertex tracks within the super RoI. The offline based algorithm reaches full efficiency only for events with more than eight vertex tracks within the super RoI. This is due to the offline based algorithm having a tighter selection on track quality. Consequently, some vertices with only a few tracks in the super RoI may not have any tracks remaining with which to form a vertex once the tracks with lower quality are removed.

The efficiency as a function of the $z$ position, seen in Fig. 41b, is approximately constant for the offline based vertex algorithm, whereas the simple histogramming algorithm shows a clear bias towards negative $z$ for the lower $E_{\mathrm{T}}$ jet trigger. This results from the very simple implementation of the histogramming algorithm, which selects the bin with the 


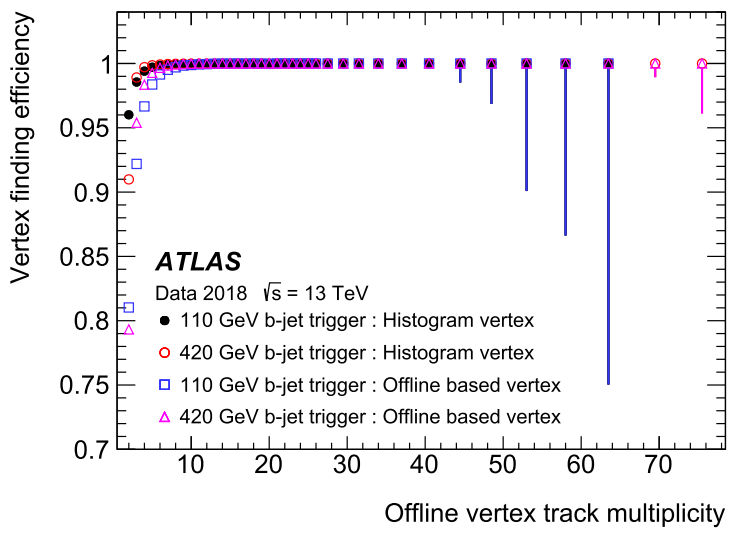

(a)

Fig. 42 The vertex algorithm efficiencies for intermediate and high $E_{\mathrm{T}} b$-jet triggers. Shown are the efficiencies a versus the offline track multiplicity for tracks in the super RoI; and $\mathbf{b}$ versus the mean pile-

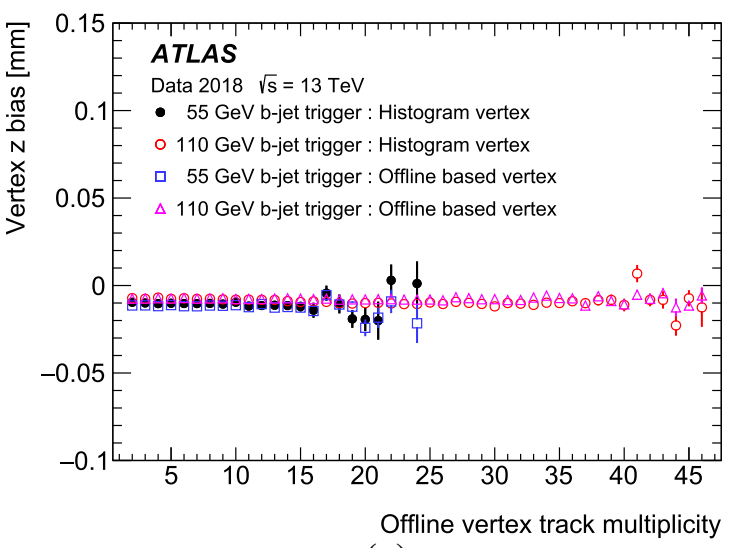

(a)

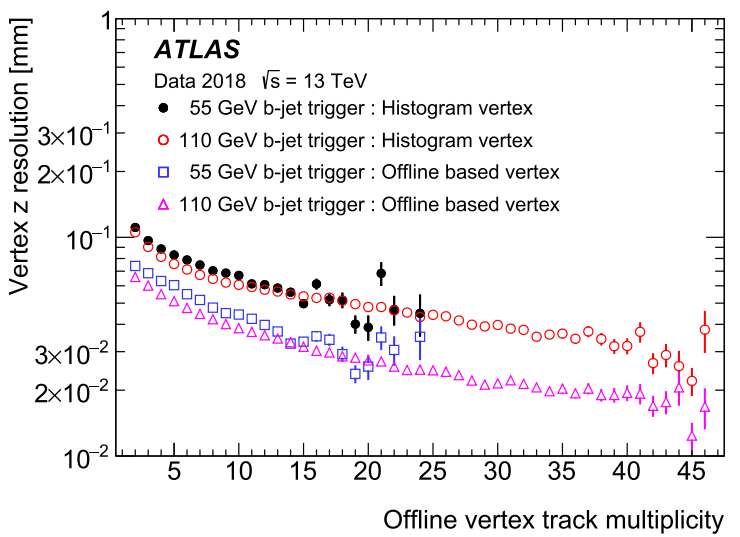

(c)

Fig. 43 The trigger vertex algorithm bias and resolution in the $z$ position along the beamline, for low and intermediate $E_{\mathrm{T}} b$-jet triggers. Shown are the $\mathbf{a}$ bias, and $\mathbf{c}$ resolution with respect to the multiplicity of

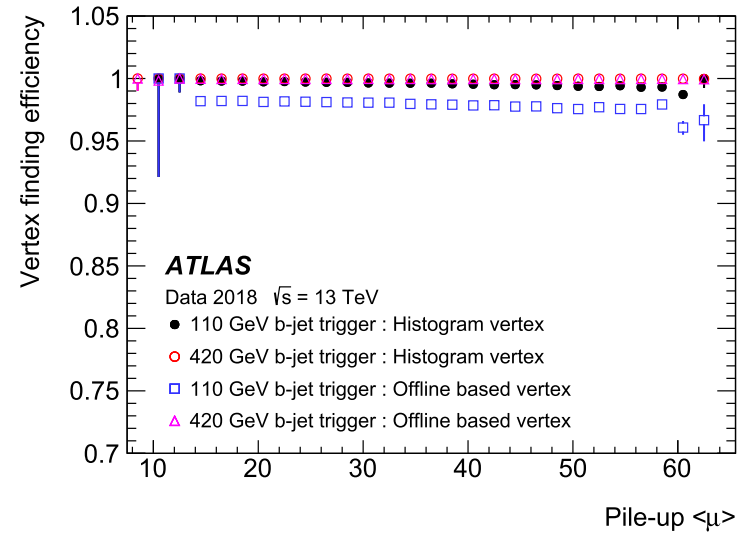

(b)

upinteraction multiplicity. Statistical Bayesian uncertainties are shown, using a $68.3 \%$ confidence interval and uniform beta function prior

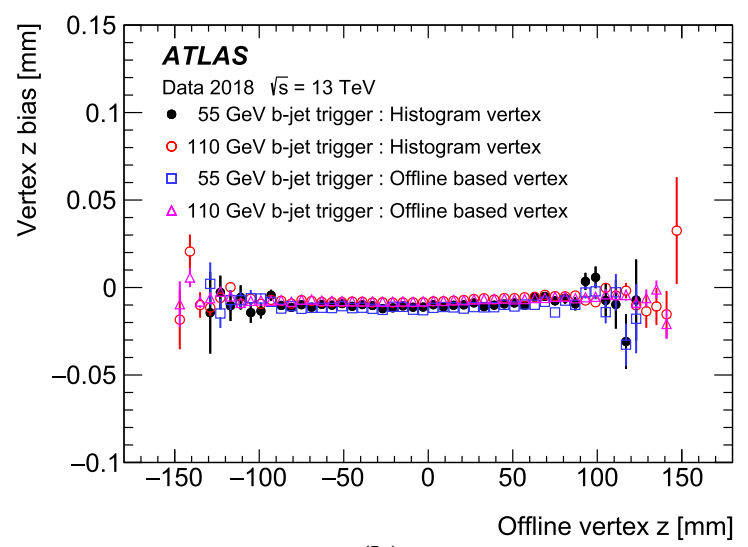

(b)

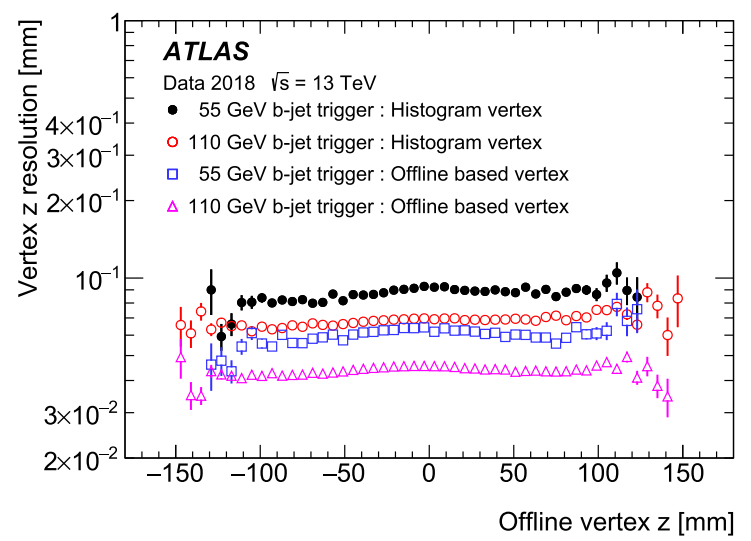

(d)

offline tracks within the super RoI which are matched to the vertex, and the $\mathbf{b}$ bias, and $\mathbf{d}$ resolution, with respect to the offline vertex $z$ position 


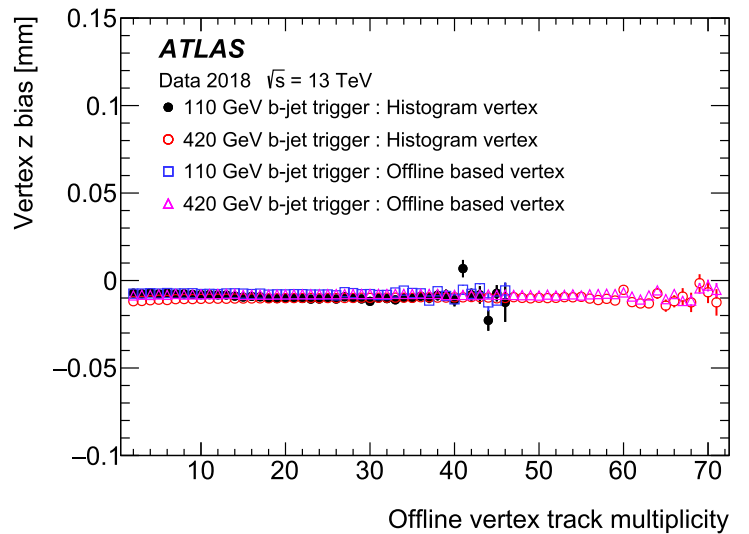

(a)

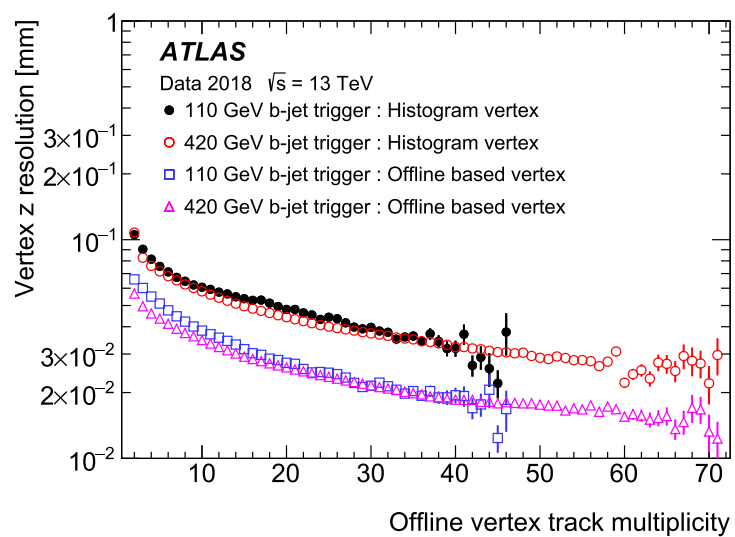

(c)

Fig. 44 The bias and resolution in the $z$ position along the beam line for the trigger vertex reconstruction for intermediate and high $E_{\mathrm{T}} b$-jet triggers. Shown are the bias (a), and resolution (c) with respect to the mul-

highest occupancy starting from the most negative $z$ bin. If it subsequently finds a bin with the same occupancy, it keeps the original bin. In low $E_{\mathrm{T}}$ events, the chance of some additional pile-up interaction having the same track multiplicity in the super RoI can be reasonably high and consequently, if such a vertex is at a more negative $z$ than the interaction of interest then the incorrect vertex can be reconstructed online, leading to a lower efficiency for vertices with a more positive- $z$ position.

For the higher $E_{\mathrm{T}}$ jet triggers shown in Figs. 41 and 42, the larger jet $E_{\mathrm{T}}$ results in significantly higher track multiplicities and larger average track $p_{\mathrm{T}}$ such that the probability of the track multiplicity for a pile-up vertex matching or exceeding that for the interaction of interest is significantly lower.

It should be noted that the overall lower efficiency for the online based vertex algorithm, and particularly for the lower $E_{\mathrm{T}}$ jet trigger is due to the lower overall track multiplicity in the events passing the trigger, such that the trigger is still on the rising edge of the efficiency distribution for many events. For the high $E_{\mathrm{T}}$ triggers where the multiplicity is

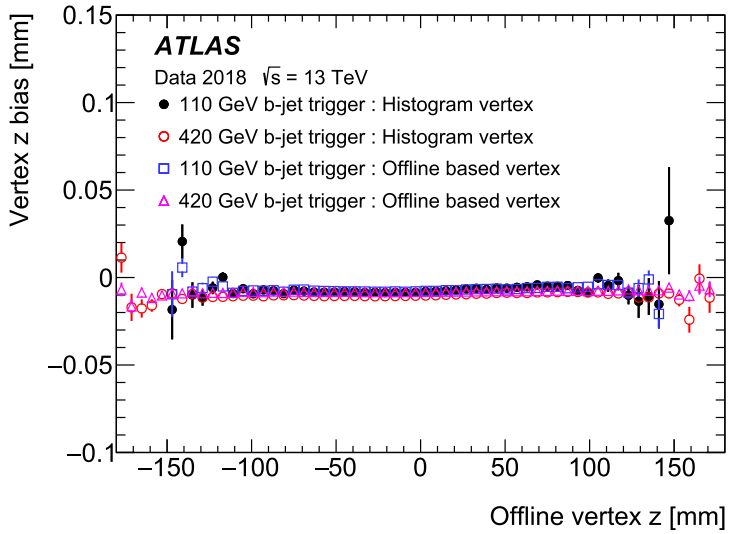

(b)

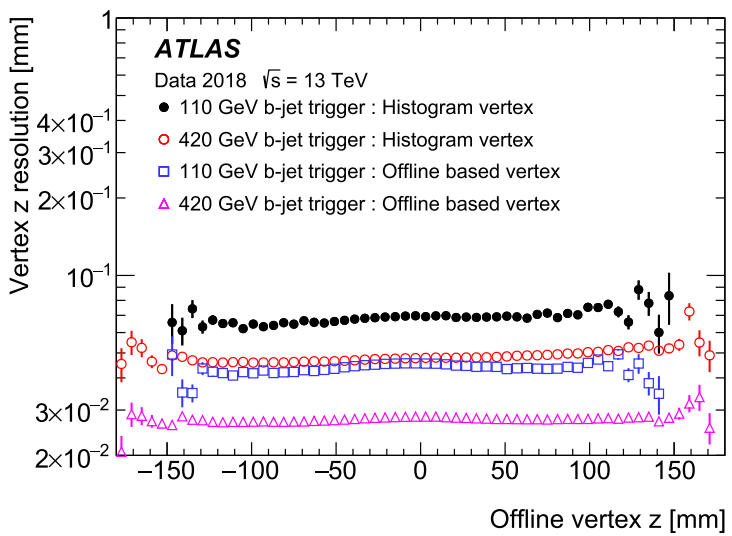

(d)

tiplicity of offline tracks on the vertex matched to within the super RoI, and the bias (b), and resolution (d), with respect to the offline vertex $z$ position

higher, the algorithm is further along the rising edge for most events. For very high $E_{\mathrm{T}}$ jet triggers, where the super RoI track multiplicity is much higher, the efficiency approaches $100 \%$. For the histogramming algorithm, the efficiency rises earlier.

Both algorithms show a decrease in efficiency as $\langle\mu\rangle$ increases, seen in Fig. 41c, with the offline based algorithm having a lower overall efficiency due to the track multiplicity of the vertex discussed above. The decrease with increasing $\langle\mu\rangle$ follows from the increased possibility for the offline selection to misidentify the jet vertex candidate within the super RoI. In this case, as the pile-up interaction multiplicity increases within an event, so do the chances of there being additional tracks from additional vertices within the super RoI that compete with the tracks from the desired primary vertex. For higher jet $E_{\mathrm{T}}$ thresholds, the track multiplicities of the desired vertex candidates is also higher, and the fall in efficiency with increasing pile-up is less pronounced. For triggers with higher still thresholds, the efficiency approaches $100 \%$ for both algorithms, and exhibits no residual loss as a 
function of pile-up. This can be seen in Fig. 42, which compares the efficiency versus pile-up $\langle\mu\rangle$ for the $420 \mathrm{GeV}$ jet trigger in addition.

Figures 43 and 44 illustrate the vertex $z$ bias and resolution for both online algorithms, where the bias is calculated as the mean of the residuals between the trigger and offline $z$ positions. Both the bias and resolution are shown as a function of either the offline track multiplicity or the offline $z$ vertex position, in Fig. 43 for the low and intermediate $E_{\mathrm{T}}$ jet triggers, and in Fig. 44 for the intermediate and high $E_{\mathrm{T}}$ jet triggers. In all cases the bias is reasonably constant and is seen to be approximately $10 \mu \mathrm{m}$ in the negative direction. This arises from a small systematic bias in the track $z$ position reconstruction. This bias is itself significantly smaller than the vertex $z$ resolution, even at high track multiplicities, and has no discernible impact on the subsequent trigger reconstruction or selection. For vertices with more positive $z$, this bias appears to decrease by a few $\mu \mathrm{m}$ for the histogramming algorithm, and less for offline based algorithm. The resolution of the vertex $z$ position for both online algorithms is seen to clearly improve with increasing track multiplicity, with the offline based algorithm showing significantly better resolution. For low multiplicity vertices the histogramming algorithm resolution is approximately $100 \mu \mathrm{m}$ whereas for the offline based algorithm it is $70 \mu \mathrm{m}$. For both algorithms the resolution improves logarithmically with track multiplicity, such that for 50 tracks the resolutions for the histogramming and offline based algorithms are approximately $30 \mu \mathrm{m}$ and $20 \mu \mathrm{m}$ respectively.

For Fig. 44 it should be noted that for the $420 \mathrm{GeV}$ high $E_{\mathrm{T}}$ jet trigger, even for low track multiplicities, the resolution is somewhat better than for the low and intermediate $E_{\mathrm{T}}$ triggers, being approximately $10 \mu \mathrm{m}$ better than for the $110 \mathrm{GeV}$ trigger, and around $20 \mu \mathrm{m}$ better than for the $55 \mathrm{GeV}$ trigger. This is a consequence of the larger average $p_{\mathrm{T}}$ of tracks from the higher $E_{\mathrm{T}}$ jet triggers, such that the track $z$ positions themselves have an intrinsically better $z$ resolution.

The resolution is largely constant with the offline vertex $z$ position with perhaps a small deterioration of the resolution around $z=0 \mathrm{~mm}$, and a slight trend towards better resolutions for more negative $z$ for the lower $E_{\mathrm{T}}$ triggers, notably for the histogramming algorithm.

\section{Conclusion}

The ATLAS inner detector trigger was modified significantly in order to operate with the extremely demanding conditions in Run 2. The many improvements in the inner detector trigger are described, and the performance is reported here for the 2016, 2017, and 2018 data recorded by ATLAS from $13 \mathrm{TeV}$ proton-proton collisions with $25 \mathrm{~ns}$ bunch spacing at the LHC. The performance has been excellent and exceeded expectations, even at the very high interaction multiplicities observed at the end of data taking in 2018. The detailed efficiencies and resolutions of the trigger in a wide range of physics signatures are presented for the full Run 2 highluminosity data set, showing the continued high efficiency, and in particular the robustness in the face of increasing pileup interaction multiplicities. The excellent performance of the inner detector trigger algorithms demonstrates how the inner detector trigger continues to lie at the heart of the trigger performance, playing an essential rôle in the ATLAS physics programme, and will continue to do so in the future.

Acknowledgements The authors thank CERN for the very successful operation of the LHC, as well as the support staff from the institutions without whom ATLAS could not be operated efficiently. The authors also gratefully acknowledge the support of ANPCyT, Argentina; YerPhI, Armenia; ARC, Australia; BMWFW and FWF, Austria; ANAS, Azerbaijan; SSTC, Belarus; CNPq and FAPESP, Brazil; NSERC, NRC and CFI, Canada; CERN; ANID, Chile; CAS, MOST and NSFC, China; Minciencias, Colombia; MSMT CR, MPO CR and VSC CR, Czech Republic; DNRF and DNSRC, Denmark; IN2P3-CNRS and CEA-DRF/IRFU, France; SRNSFG, Georgia; BMBF, HGF and MPG, Germany; GSRI, Greece; RGC and Hong Kong SAR, China; ISF and Benoziyo Center, Israel; INFN, Italy; MEXT and JSPS, Japan; CNRST, Morocco; NWO, Netherlands; RCN, Norway; MEiN, Poland; FCT, Portugal; MNE/IFA, Romania; JINR; MES of Russia and NRC KI, Russian Federation; MESTD, Serbia; MSSR, Slovakia; ARRS and MIZŠ, Slovenia; DSI/NRF, South Africa; MICINN, Spain; SRC and Wallenberg Foundation, Sweden; SERI, SNSF and Cantons of Bern and Geneva, Switzerland; MOST, Taiwan; TAEK, Turkey; STFC, United Kingdom; DOE and NSF, United States of America. In addition, individual groups and members have received support from BCKDF, CANARIE, Compute Canada and CRC, Canada; COST, ERC, ERDF, Horizon 2020 and Marie Skłodowska-Curie Actions, European Union; Investissements d'Avenir Labex, Investissements d'Avenir Idex and ANR, France; DFG and AvH Foundation, Germany; Herakleitos, Thales and Aristeia programmes co-financed by EU-ESF and the Greek NSRF, Greece; BSFNSF and GIF, Israel; Norwegian Financial Mechanism 2014-2021, Norway; NCN and NAWA, Poland; La Caixa Banking Foundation, CERCA Programme Generalitat de Catalunya and PROMETEO and GenT Programmes Generalitat Valenciana, Spain; Göran Gustafssons Stiftelse, Sweden; The Royal Society and Leverhulme Trust, United Kingdom. The crucial computing support from all WLCG partners is acknowledged gratefully, in particular from CERN, the ATLAS Tier-1 facilities at TRIUMF (Canada), NDGF (Denmark, Norway, Sweden), CCIN2P3 (France), KIT/GridKA (Germany), INFN-CNAF (Italy), NLT1 (Netherlands), PIC (Spain), ASGC (Taiwan), RAL (UK) and BNL (USA), the Tier-2 facilities worldwide and large non-WLCG resource providers. Major contributors of computing resources are listed elsewhere [67].

Data Availability Statement This manuscript has no associated data or the data will not be deposited. [Authors' comment: All ATLAS scientific output is published in journals, and preliminary results are made available in Conference Notes. All are openly available, without restriction on use by external parties beyond copyright law and the standard conditions agreed by CERN. Data associated with journal publications are also made available: tables and data from plots (e.g. cross section values, likelihood profiles, selection efficiencies, cross section limits, ...) are stored in appropriate repositories such as HEPDATA (http:// hepdata.cedar.ac.uk/). ATLAS also strives to make additional material related to the paper available that allows a reinterpretation of the data in the context of new theoretical models. For example, an extended 
encapsulation of the analysis is often provided for measurements in the framework of RIVET (http://rivet.hepforge.org/). This information is taken from the ATLAS Data Access Policy, which is a public document that can be downloaded from http://opendata.cern.ch/record/413 [opendata.cern.ch].]

Open Access This article is licensed under a Creative Commons Attribution 4.0 International License, which permits use, sharing, adaptation, distribution and reproduction in any medium or format, as long as you give appropriate credit to the original author(s) and the source, provide a link to the Creative Commons licence, and indicate if changes were made. The images or other third party material in this article are included in the article's Creative Commons licence, unless indicated otherwise in a credit line to the material. If material is not included in the article's Creative Commons licence and your intended use is not permitted by statutory regulation or exceeds the permitted use, you will need to obtain permission directly from the copyright holder. To view a copy of this licence, visit http://creativecomm ons.org/licenses/by/4.0/.

Funded by SCOAP ${ }^{3}$.

\section{References}

1. L. Evans, P. Bryant, L.H.C. Machine, JINST 3, S08001 (2008). https://doi.org/10.1088/1748-0221/3/08/S08001

2. ATLAS Collaboration, The ATLAS Experiment at the CERN Large Hadron Collider. JINST 3, S08003 (2008) https://doi.org/10.1088/ 1748-0221/3/08/S08003

3. ATLAS Collaboration, Performance of the ATLAS Trigger System in 2010, Eur. Phys. J. C 72, 1849 (2012). arXiv:1110.1530 [hep-ex]

4. ATLAS Collaboration, Performance of the ATLAS trigger system in 2015. Eur. Phys. J. C 77, 317 (2017). arXiv:1611.09661 [hep-ex]

5. ATLAS Collaboration, Performance of electron and photon triggers in ATLAS during LHC Run 2. Eur. Phys. J. C 80, 47 (2020). arXiv:1909.00761 [hep-ex]

6. ATLAS Collaboration, Performance of the ATLAS muon trigger in pp collisions at $\sqrt{s}=8 T e V$. Eur. Phys. J. C 75, 120 (2015). arXiv: 1408.3179 [hep-ex]

7. ATLAS Collaboration, The ATLAS Tau Trigger in Run 2, ATLASCONF-2017-061 (2017). https://cds.cern.ch/record/2274201

8. ATLAS Collaboration, Identification and energy calibration of hadronically decaying tau leptons with the ATLAS experiment in pp collisions at $\sqrt{s}=8 T e V$. Eur. Phys. J. C 75, 303 (2015). arXiv:1412.7086 [hep-ex]

9. ATLAS Collaboration, The performance of the jet trigger for the ATLAS detector during 2011 data taking. Eur. Phys. J. C 76, 526 (2016). arXiv:1606.07759 [hep-ex]

10. ATLAS Collaboration, Configuration and performance of the ATLAS $b$-jet triggers in Run 2. Eur. Phys. J. C 81, 1087 (2021). https://doi.org/10.1140/epjc/s10052-021-09775-5. arXiv:2106.03584 [hep-ex]

11. ATLAS Collaboration, Electron reconstruction and identification in the ATLAS experiment using the 2015 and 2016 LHC protonproton collision data at $\sqrt{s}=13 \mathrm{TeV}$. Eur. Phys. J. C 79, 639 (2019). arXiv:1902.0465 5 [hep-ex]

12. ATLAS Collaboration, Measurement of the muon reconstruction performance of the ATLAS detector using 2011 and 2012 LHC proton-proton collision data. Eur. Phys. J. C 74, 3130 (2014). arXiv:1407.3935 [hep-ex]

13. ATLAS Collaboration, Reconstruction of hadronic decay products of tau leptons with the ATLAS experiment. Eur. Phys. J. C 76, 295 (2016). arXiv:1512.05955 [hep-ex]
14. ATLAS Collaboration, Performance of b-jet identification in the ATLAS experiment. JINST 11, P04008 (2016). arXiv:1512.01094 [hep-ex]

15. ATLAS Collaboration, ATLAS b-jet identification performance and efficiency measurement with $t \bar{t}$ events in pp collisions at $\sqrt{s}=13$ TeV. Eur. Phys. J. C 79, 970 (2019). arXiv:1907.05120 [hep-ex]

16. ATLAS Collaboration, Studies for the development of the inner detector trigger algorithms at ATLAS, ATL-DAQ-PUB-2013-002 (2013). https://cds.cern.ch/record/1602918

17. ATLAS Collaboration, ATLAS Insertable B-Layer Technical Design Report, ATLAS-TDR-19; CERN-LHCC-2010-013 (2010). https://cds.cern.ch/record/1291633 [Addendum: ATLAS-TDR19-ADD-1; CERN-LHCC-2012-009 (2012) https://cds.cern.ch/ record/1451888]

18. B. Abbott et al., Production and integration of the ATLAS Insertable B-Layer. JINST 13, T05008 (2018). arXiv:1803.00844 [physics.ins-det]

19. ATLAS Collaboration, ATLAS pixel detector electronics and sensors. JINST 3, P07007 (2008) https://doi.org/10.1088/1748-0221/ 3/07/p07007

20. A. Ahmad et al., The silicon microstrip sensors of the ATLAS semiconductor tracker. Nucl. Instrum. Meth. A 578, 98 (2007). https://doi.org/10.1016/j.nima.2007.04.157

21. E. Abat et al., The ATLAS Transition Radiation Tracker (TRT) proportional drift tube: design and performance. JINST 3, P02013 (2008). https://doi.org/10.1088/1748-0221/3/02/P02013

22. ATLAS Collaboration, Basic ATLAS TRT performance studies of Run 1, ATL-INDET-PUB-2014-001 (2014). https://cds.cern.ch/ record/1669603

23. ATLAS Collaboration, Performance of the ATLAS Transition Radiation Tracker in Run 1 of the LHC: tracker properties. JINST 12, P05002 (2017). arXiv: 1702.06473 [hep-ex]

24. ATLAS Collaboration, ATLAS Muon Spectrometer: Technical Design Report, ATLAS-TDR-10; CERN-LHCC-97-022, CERN (1997). https://cds.cern.ch/record/331068

25. ATLAS Collaboration, Performance of the ATLAS muon triggers in Run 2. JINST 15 P09015 (2020). arXiv:2004.13447 [hep-ex]

26. ATLAS Collaboration, ATLAS Level-1 Trigger: Technical Design Report, ATLAS-TDR-12; CERN-LHCC-98-014 (1998). https:// cds.cern.ch/record/381429

27. ATLAS Collaboration, Operation of the ATLAS trigger system in Run 2. JINST 15, P10004 (2020). arXiv:2007.12539 [physics.insdet]

28. ATLAS TDAQ Collaboration, The ATLAS Data Acquisition and High Level Trigger system. JINST 11, P06008 (2016) https://doi. org/10.1088/1748-0221/11/06/P06008

29. ATLAS Collaboration, ATLAS High-Level Trigger, Data Acquisition and Controls: Technical Design Report, ATLAS-TDR16; CERN-LHCC-2003-022 (2003). https://cds.cern.ch/record/ 616089

30. ATLAS Collaboration, Luminosity determination in pp collisions at $\sqrt{s}=13 \mathrm{TeV}$ using the ATLAS detector at the LHC, ATLASCONF-2019-021 (2019). https://cds.cern.ch/record/2677054

31. N. Berger et al., The ATLAS high level trigger steering. J. Phys. Conf. Ser. 119, 022013 (2008). https://doi.org/10.1088/ 1742-6596/119/2/022013

32. H.P. Beck et al., Performance of the final Event Builder for the ATLAS Experiment. IEEE Trans. Nucl. Sci. 55, 176 (2008). https:// doi.org/10.1109/TNS.2007.910868

33. ATLAS Collaboration, Technical Design Report for the Phase-I Upgrade of the ATLAS TDAQ System, ATLAS-TDR-023 (2013). http://cdsweb.cern.ch/record/1602235

34. N. Konstantinidis et al., A fast tracking algorithm for the ATLAS level 2 trigger. Nucl. Instrum. Meth. A 566, 166 (2006) TIME 2005 https://doi.org/10.1016/j.nima.2006.05.059 
35. ATLAS Collaboration, Performance of the ATLAS Inner Detector Trigger algorithms in $\mathrm{p}-\mathrm{p}$ collisions at $\sqrt{s}=900 \mathrm{GeV}$, ATLASCONF-2010-014 (2010). https://cds.cern.ch/record/1276548

36. R.E. Kalman, A new approach to linear filtering and prediction problems. J. Fluids Eng. 82, 35 (1960). https://doi.org/10.1115/1. 3662552

37. T. Cornelissen et al., The new ATLAS track reconstruction (NEWT). J. Phys. Conf. Ser. 119, 032014 (2008). https://doi.org/ $10.1088 / 1742-6596 / 119 / 3 / 032014$

38. A. Salzburger, Optimisation of the ATLAS Track Reconstruction Software for Run-2. J. Phys. Conf. Ser. 664, 072042 (2015). https:// doi.org/10.1088/1742-6596/664/7/072042

39. N. Styles, M. Bellomo, A. Salzburger, Developments in the ATLAS Tracking Software ahead of LHC Run 2. J. Phys. Conf. Ser. 608, 012047 (2015) (ed. by L. Fiala, M. Lokajicek and N. Tumova) https://doi.org/10.1088/1742-6596/608/1/012047

40. L. Lonnblad, CLHEP - a project for designing a C++ class library for high-energy physics. Comput. Phys. Commun. 84, 307 (1994). https://doi.org/10.1016/0010-4655(94)90217-8

41. G. Guennebaud, B. Jacob et al., Eigen v3 (2010). http://eigen. tuxfamily.org

42. ATLAS Collaboration, Operation and performance of the ATLAS semiconductor tracker. JINST 9, P08009 (2014). arXiv:1404.7473 [hep-ex]

43. V. Boisvert et al., A new implementation of the region of interest strategy for the ATLAS second level trigger, ECONFC0303241:TUGT008 (2003). arXiv:physics/0306101 [physics.comp-ph]

44. ATLAS Collaboration, Alignment of the ATLAS Inner Detector in Run 2. Eur. Phys. J. C 80, 1194 (2020). arXiv:2007.07624 [hep-ex]

45. P. Yepes, A fast track pattern recognition. Nucl. Instrum. Meth. A380, 582 (1996). https://doi.org/10.1016/ 0168-9002(96)00726-7

46. ATLAS Collaboration, Performance of the ATLAS track reconstruction algorithms in dense environments in LHC Run 2. Eur. Phys. J. C 77, 673 (2017). arXiv:1704.07983 [hep-ex]

47. R. Fruhwirth, Application of Kalman filtering to track and vertex fitting. Nucl. Instrum. Meth. A262, 444 (1987). https://doi.org/10. 1016/0168-9002(87)90887-4

48. ATLAS Collaboration, Performance of the ATLAS silicon pattern recognition algorithm in data and simulation at $\sqrt{s}=$ $7 \mathrm{TeV}$, ATLAS-CONF-2010-072 (2010). https://cds.cern.ch/ record/1281363

49. ATLAS Collaboration, ATLAS Inner Detector: Technical Design Report, Vol. 1, ATLAS-TDR-4; CERN-LHCC-97-016 (1997). https://cds.cern.ch/record/331063

50. ATLAS Collaboration, ATLAS Inner Detector: Technical Design Report, Vol. 2, ATLAS-TDR-5, CERN-LHCC-97-017 (1997). https://cds.cern.ch/record/331064

51. T.G. Cornelissen et al., The global $x^{2}$ track fitter in ATLAS. J. Phys. Conf. Ser. 119, 032013 (2008). https://doi.org/10.1088/ $1742-6596 / 119 / 3 / 032013$

52. P. Ferrari, Tracking and vertexing at ATLAS, 17th Symposium on Hadron Collider Physics 2006 (HCP 2006) (2007). arXiv:0707.3071 [physics.ins-det]

53. ATLAS Collaboration, Reconstruction of primary vertices at the ATLAS experiment in Run 1 proton-proton collisions at the LHC. Eur. Phys. J. C 77, 332 (2017). arXiv:1611.10235 [hep-ex]

54. ATLAS Collaboration, Performance of the ATLAS trigger system in 2010. Eur. Phys. J. C 72, 1849 (2012). arXiv:1110.1530 [hep-ex]

55. F. Meloni, Primary vertex reconstruction with the ATLAS detector. JINST 11, C12060 (2016). https://doi.org/10.1088/1748-0221/11/ $12 / \mathrm{c} 12060$

56. ATLAS Collaboration, Jet energy scale and resolution measured in proton-proton collisions at $\sqrt{s}=13 \mathrm{TeV}$ with the ATLAS detector. Eur. Phys. J. C 81, 689 (2020). arXiv:2007.02645 [hep-ex]
57. ATLAS Collaboration, Determination of jet calibration and energy resolution in proton-proton collisions at $\sqrt{s}=8 \mathrm{TeV}$ using the ATLAS detector. Eur. Phys. J. C 80, 1104 (2020). arXiv:1910.04482 [hep-ex]

58. ATLAS Collaboration, Jet energy scale and resolution measured in proton-proton collisions at $\sqrt{s}=13 \mathrm{TeV}$ with the ATLAS detector (2020). arXiv:2007.02645 [hep-ex]

59. W. Lampl et al., Calorimeter clustering algorithms: description and performance, ATL-LARG-PUB-2008-002 (2008). https://cds. cern.ch/record/1099735

60. ATLAS Collaboration, Standalone vertex finding in the ATLAS muon spectrometer. JINST 9, P02001 (2014). arXiv:1311.7070 [hep-ex]

61. ATLAS Collaboration, ATLAS data quality operations and performance for 2015-2018 data-taking. JINST 15, P04003 (2020). arXiv:1911.04632 [physics.ins-det]

62. D. Gale, L.S. Shapley, College admissions and the stability of marriage. Am. Math. Mon. 69, 9 (1962). http://www.jstor.org/stable/ 2312726

63. ATLAS Collaboration, Muon reconstruction performance of the ATLAS detector in proton-proton collision data at $\sqrt{s}=13 \mathrm{TeV}$. Eur. Phys. J. C 76, 292 (2016). arXiv:1603.05598 [hep-ex]

64. ATLAS Collaboration, Improved electron reconstruction in ATLAS using the Gaussian sum filter-based model for bremsstrahlung, ATLAS-CONF-2012-047 (2012). https://cds. cern.ch/record/1449796

65. ATLAS Collaboration, Trigger menu in 2017, ATL-DAQ-PUB2018-002 (2018). https://cds.cern.ch/record/2625986

66. S. Boutle et al., Primary vertex reconstruction at the ATLAS experiment. J. Phys. Conf. Ser. 898, 042056 (2017). https://doi.org/10. 1088/1742-6596/898/4/042056

67. ATLAS Collaboration, ATLAS computing acknowledgements, ATL-SOFT-PUB-2021-003. https://cds.cern.ch/record/2776662 


\section{ATLAS Collaboration}

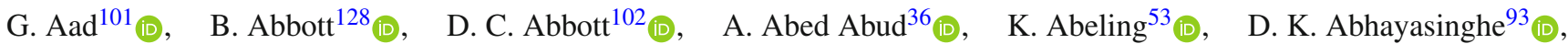
S. H. Abidi ${ }^{29}$ (1), O. S. AbouZeid ${ }^{40}$ (D), N. L. Abraham ${ }^{156}, \quad$ H. Abramowicz ${ }^{161}$ (D), H. Abreu ${ }^{160}\left(0_{0}, \quad\right.$ Y. Abulaiti ${ }^{6}$ (D), B. S. Acharya ${ }^{66 a, 66 b, 0}{ }_{(\mathbb{D})}$, B. $\operatorname{Achkar}^{53}$ (D) L. Adam $^{99}$ (D), C. Adam Bourdarios ${ }^{5}$ (D) L. Adamczyk ${ }^{83 a}$ (D) L. Adamek ${ }^{166}$ (D),

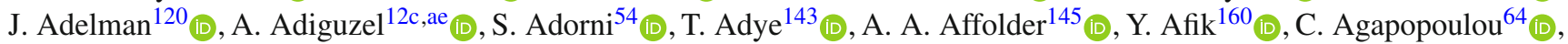

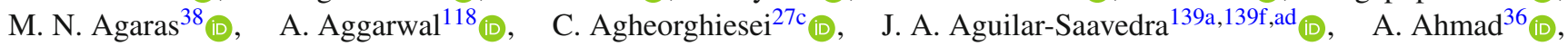

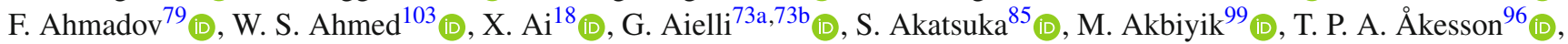
E. Akilli ${ }^{54}$ (D) A. V. Akimov ${ }^{110}$ (D) K. Al Khoury ${ }^{64}$ (D) , G. L. Alberghi ${ }^{23 b}$ (D) J. Albert $^{175}$ (D), M. J. Alconada Verzini ${ }^{161}$ (D), S. Alderweireldt ${ }^{36}$ (D), M. Aleksa ${ }^{36}$ (I), I. N. Aleksandrov ${ }^{79}$ (D), C. Alexa ${ }^{27 b}$ (I), T. Alexopoulos ${ }^{10}$ (D), A. Alfonsi ${ }^{119}$ (D),

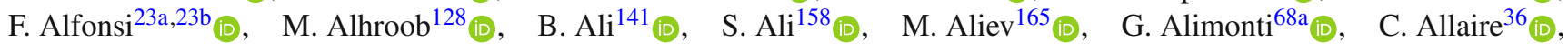

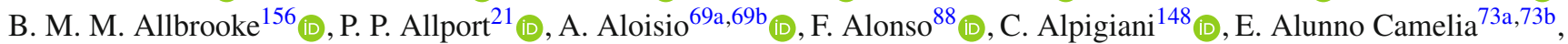

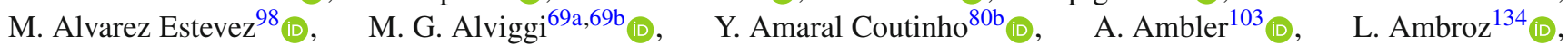

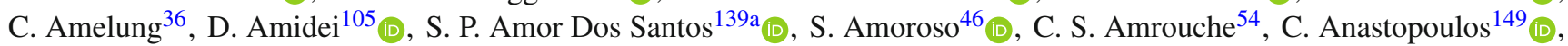

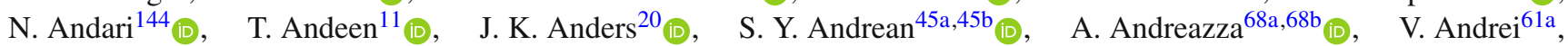

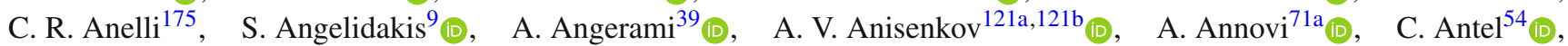

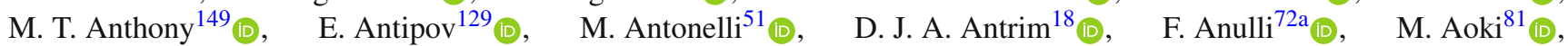

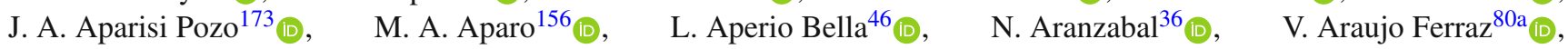
R. Araujo Pereira ${ }^{80 b}$, C. Arcangeletti $^{51}$ (D), A. T. H. $\operatorname{Arce}^{49}$ (D), J-F. Arguin ${ }^{109}$ (D), S. Argyropoulos ${ }^{52}$ (D), J.-H. Arling ${ }^{46}$ (D),

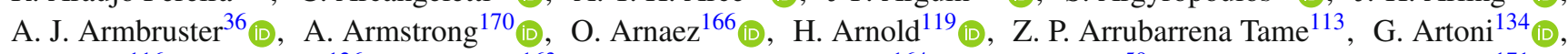

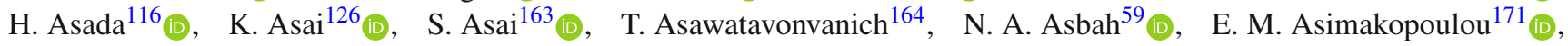

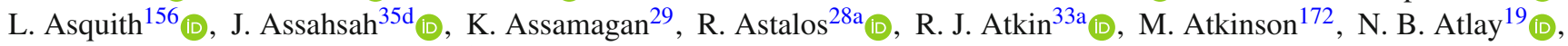
H. Atmani ${ }^{64}$, P. A. Atmasiddha ${ }^{105}$ (D), K. Augsten ${ }^{141}$ (D), V. A. Austrup ${ }^{181}$ (D), G. Avolio $^{36}$ (D), M. K. Ayoub ${ }^{15 c}$ (D),

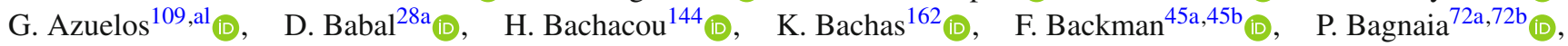
H. Bahrasemani ${ }^{152}$, A. J. Bailey ${ }^{173}$ (D), V. R. Bailey ${ }^{172}$ (D), J. T. Baines ${ }^{143}$ (D), C. Bakalis ${ }^{10}$ (D), O. K. Baker ${ }^{182}$ (D),

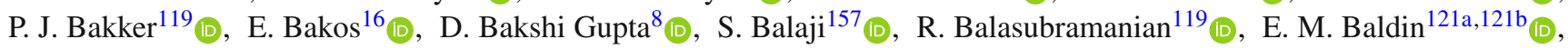

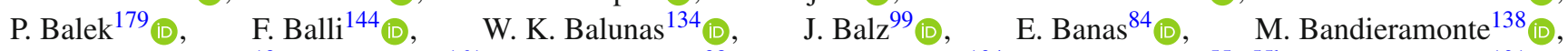
A. Bandyopadhyay ${ }^{19}$ (D), L. Barak ${ }^{161}$ (D), W. M. Barbe ${ }^{38}$ (D) E. L. Barberio ${ }^{104}$ (D), D. Barberis ${ }^{55 a, 55 b}$ (D), M. Barbero ${ }^{101}$ (D),

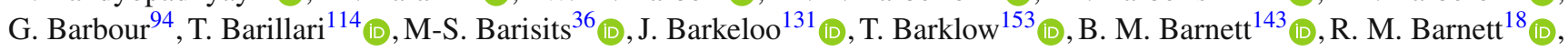

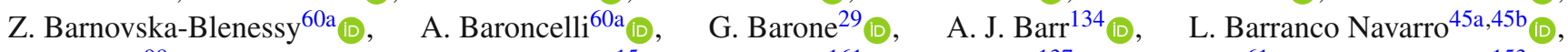

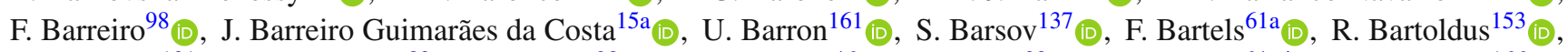

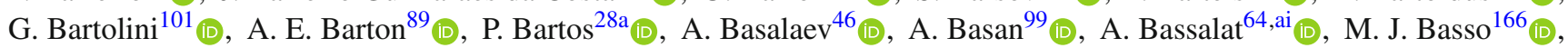

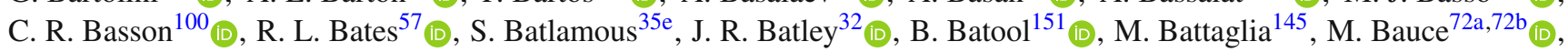
F. Bauer ${ }^{144, *}$ (D) P. Bauer ${ }^{24}$ (D) H. S. Bawa ${ }^{31}$, A. Bayirli ${ }^{12 c}$ (D) J. B. Beacham ${ }^{49}$ (D), T. Beau ${ }^{135}$ (I) P. H. Beauchemin ${ }^{169}$ (D),

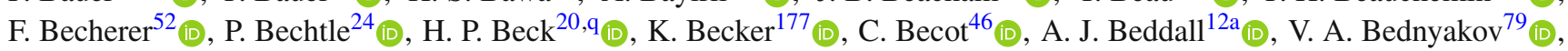

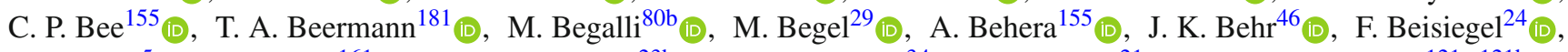

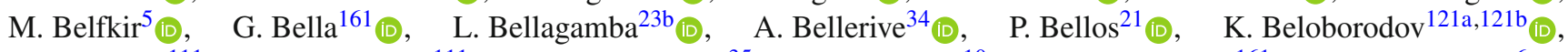

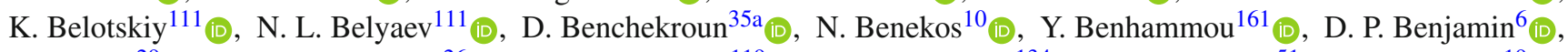

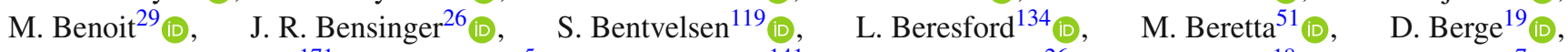
E. Bergeaas Kuutmann ${ }^{171}$ (D), N. Berger ${ }^{5}$ (D), B. Bergmann ${ }^{141}$ (D), L. J. Bergsten ${ }^{26}$ (D), J. Beringer ${ }^{18}$ (D), S. Berlendis ${ }^{7}$ (D), G. Bernardi ${ }^{135}$ (D) C. Bernius ${ }^{153}$ (D) , F. U. Bernlochner ${ }^{24}$ (D) T. Berry ${ }^{93}$ (I) , P. Berta ${ }^{99}$ (D) A. Berthold ${ }^{48}$ (D) I. A. Bertram ${ }^{89}$ (D), O. Bessidskaia Bylund ${ }^{181}$ (D), S. Bethke ${ }^{114}$ (D), A. Betti ${ }^{42}$ (D) A. J. Bevan ${ }^{92}$ (D), S. Bhatta ${ }^{155}$ (D), D. S. Bhattacharya ${ }^{176}$ (D) $_{\text {, }}$

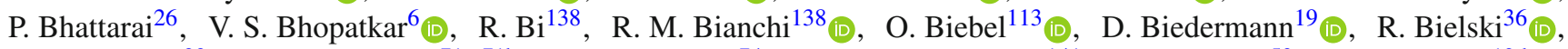

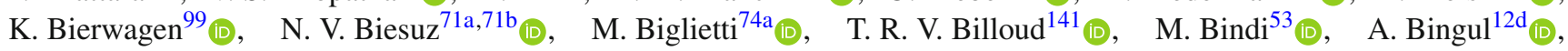

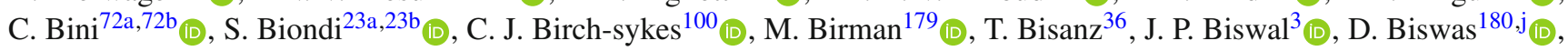

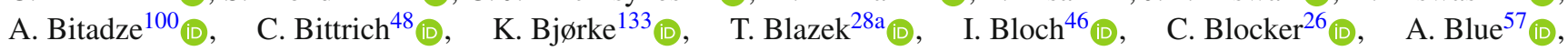

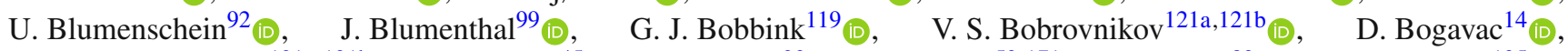

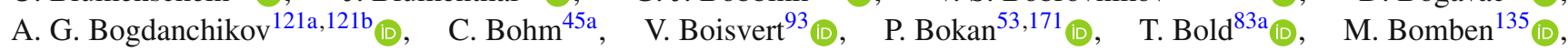

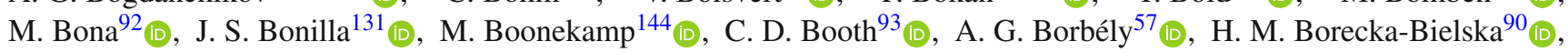

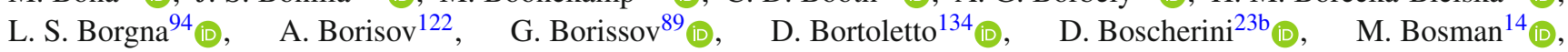

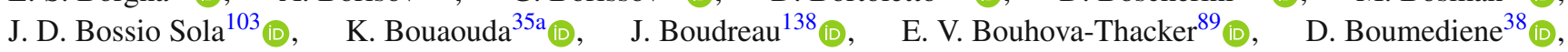
R. Bouquet ${ }^{135}$ (I), A. Boveia ${ }^{127}$ (D), J. Boyd ${ }^{36}$ (D), D. Boye ${ }^{29}$ (D), I. R. Boyko ${ }^{79}$ (D), A. J. Bozson ${ }^{93}$ (D), J. Bracinik ${ }^{21}$ (D),

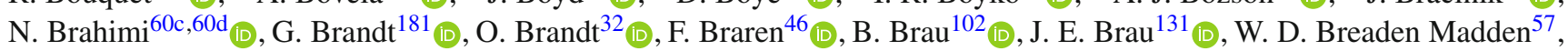




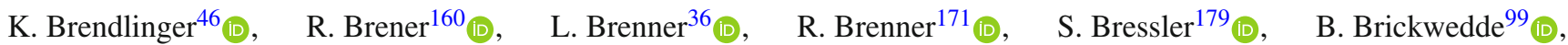
D. L. Briglin ${ }^{21}$ (D), D. Britton ${ }^{57}$ (D), D. Britzger ${ }^{114}$ (D) I. Brock ${ }^{24}$ (D) R. Brock ${ }^{106}$ (D), G. Brooijmans ${ }^{39}$ (D) W. K. Brooks ${ }^{146 f}$ (D),

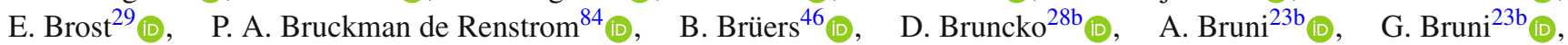

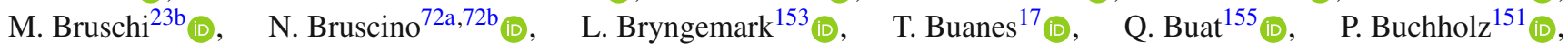
A. G. Buckley ${ }^{57}$ (D), I. A. Budagov ${ }^{79}$ (D), M. K. Bugge ${ }^{133}$ (D), O. Bulekov ${ }^{111}$ (D), B. A. Bullard ${ }^{59}$ (D), T. J. Burch ${ }^{120} \mathbb{C}_{\text {(D) }}$,

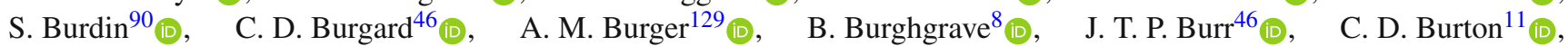

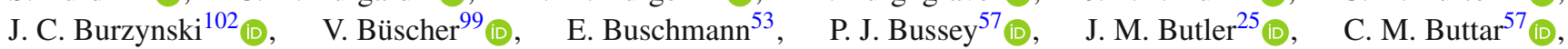

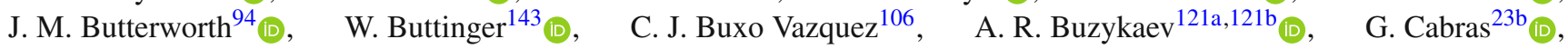

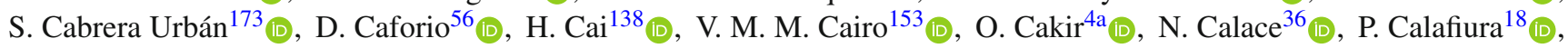
G. Calderini ${ }^{135}(\mathbb{D})$ P. Calfayan ${ }^{65}$ (D), G. Callea ${ }^{57}$ (D) L. P. Caloba ${ }^{80 b}, \quad$ A. Caltabiano ${ }^{73 a}, 73 b, \quad$ S. Calvente Lopez ${ }^{98}$ (D),

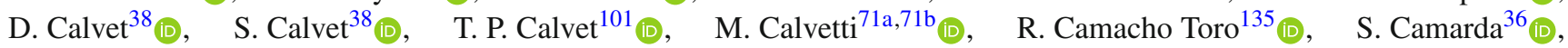
$\begin{array}{ll}\text { D. Camarero Munoz } & \text { (D) }, \quad \text { P. Camarri } \\ \text { 73a,73b } & \text { M. T. Camerlingo }\end{array}$

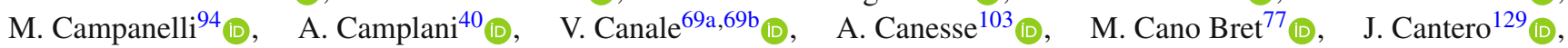

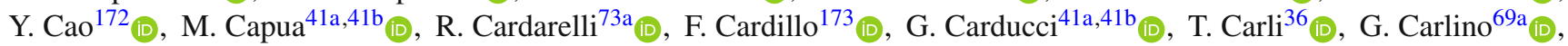

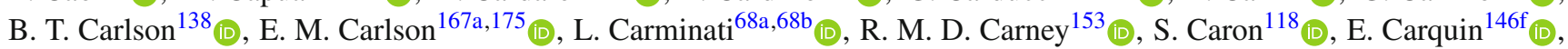

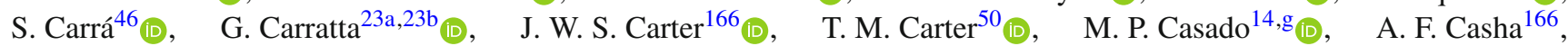

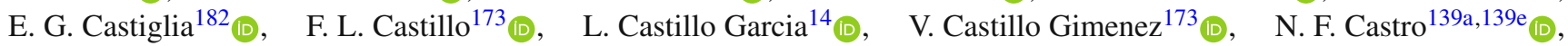

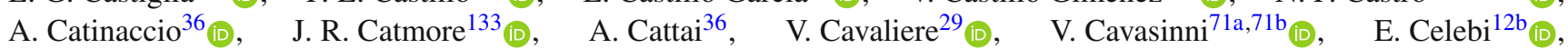

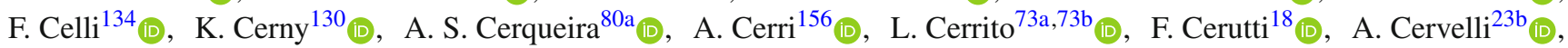

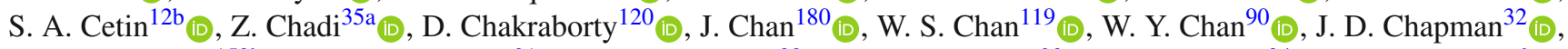
B. Chargeishvili ${ }^{159 b}$ (D), D. G. Charlton ${ }^{21}$ (D), T. P. Charman ${ }^{92}$ (D) M. Chatterjee ${ }^{20}$ (D) C. C. Chau ${ }^{34}$ (D) S. Chekanov ${ }^{6}$,

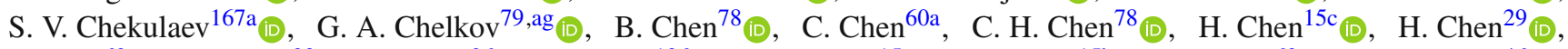

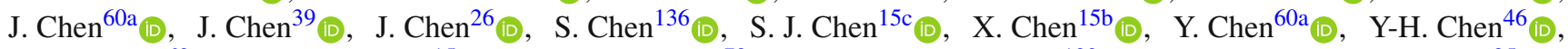

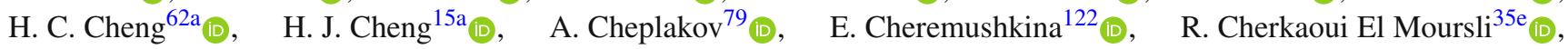

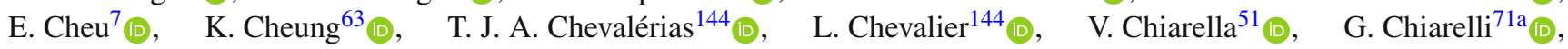

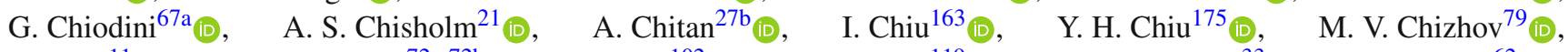

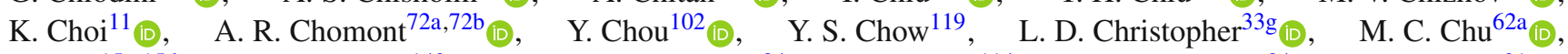

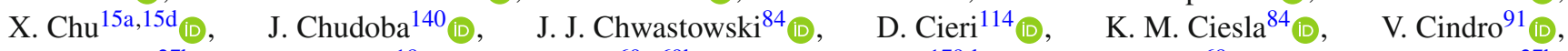

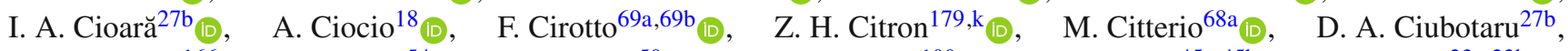

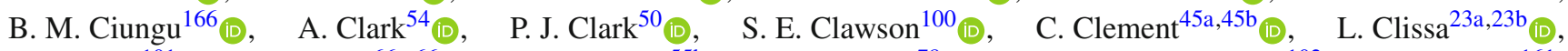
Y. Coadou ${ }^{101}$ (D), M. Cobal ${ }^{66 a, 66 c}$ (D), A. Coccaro ${ }^{55 b}$ (I),$\quad$ J. Cochran ${ }^{78}, \quad$ R. Coelho Lopes De Sa ${ }^{102}$ (D), H. Cohen ${ }^{161}$,

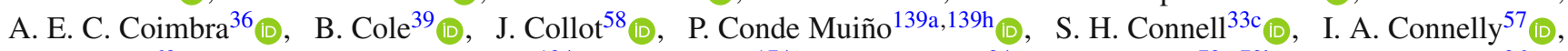

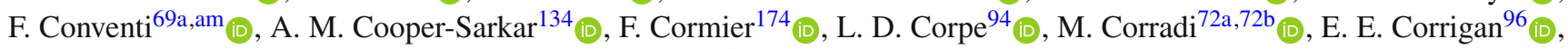

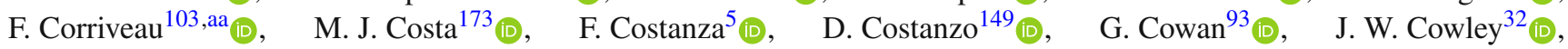

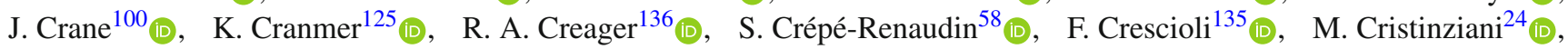

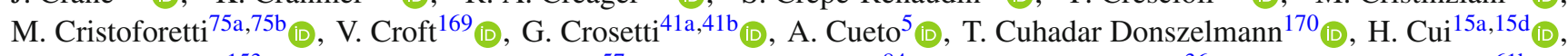

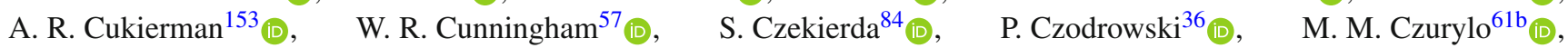

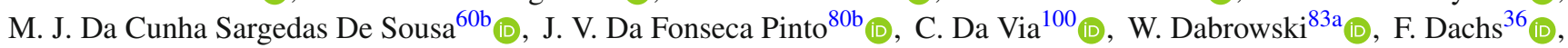

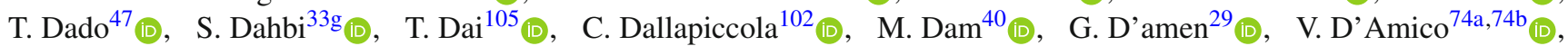

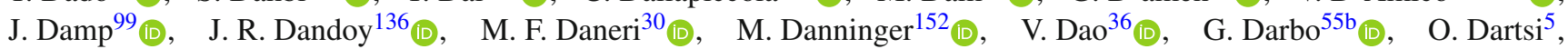

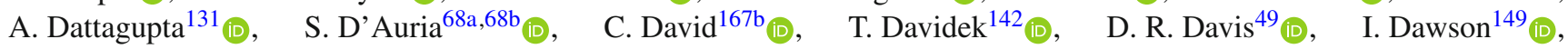

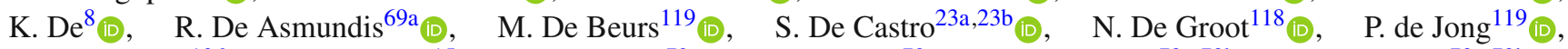

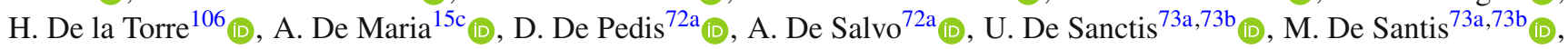

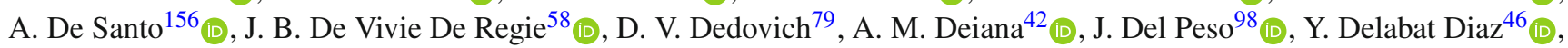

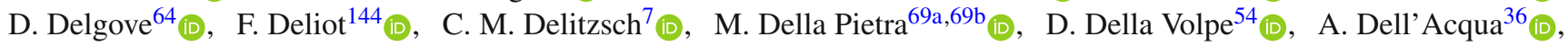

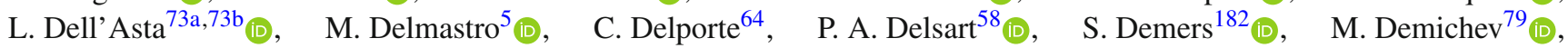

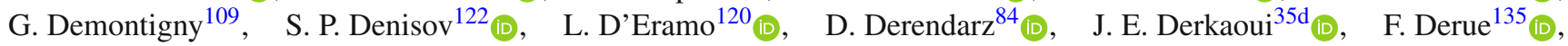

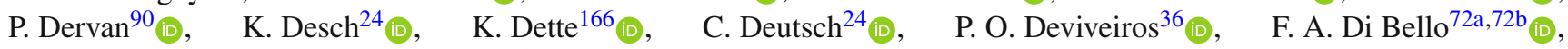

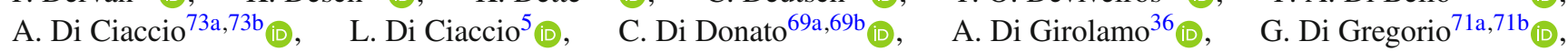

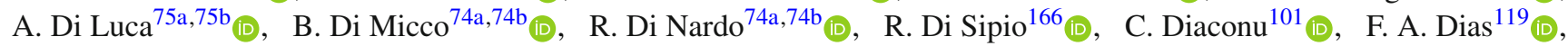

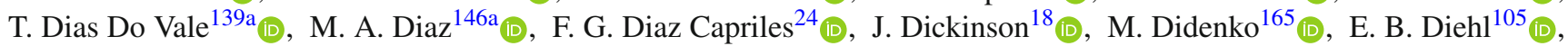

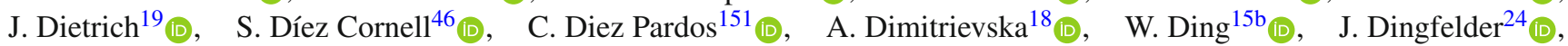

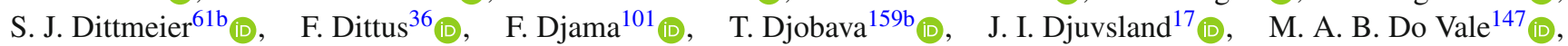




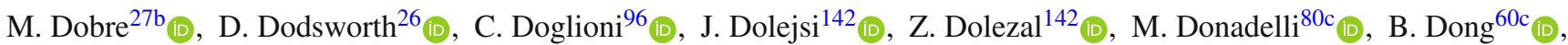
J. Donini ${ }^{38}$ (D), A. D'onofrio ${ }^{15 c_{1}}$, M. D’Onofrio ${ }^{90}$ (D), J. Dopke ${ }^{143}$ (D), A. Doria ${ }^{69} a_{\text {(D) }}$, M. T. Dova ${ }^{88}$ (D), A. T. Doyle ${ }^{57}$ (D),

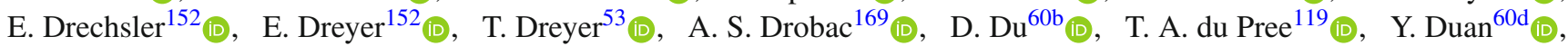

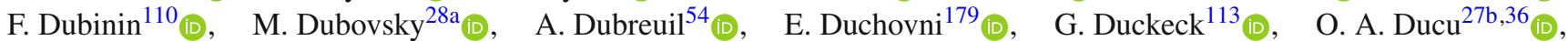

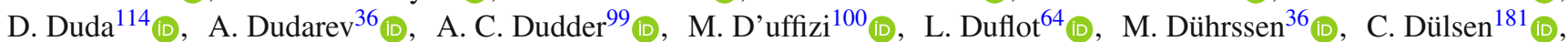

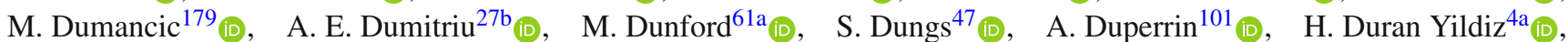

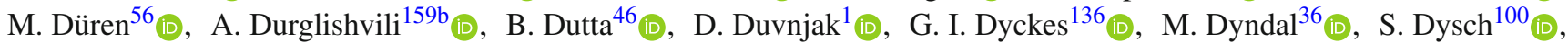

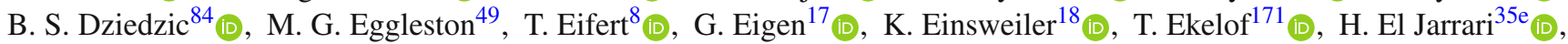

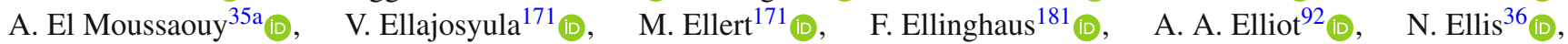

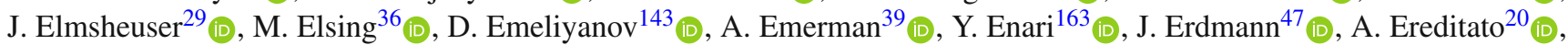

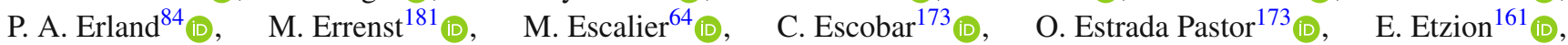

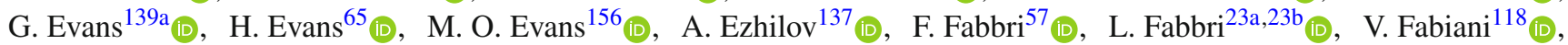

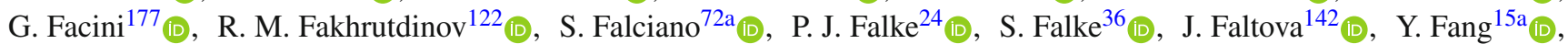

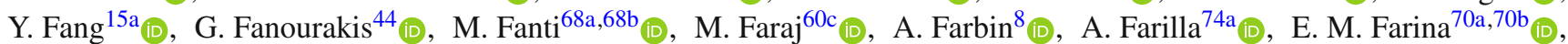

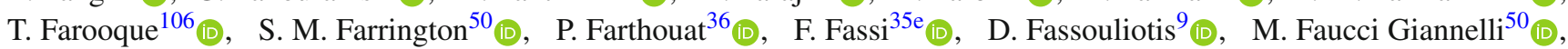

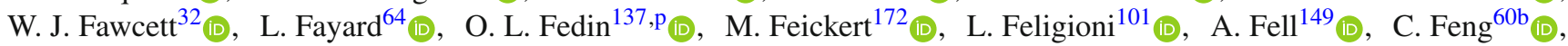

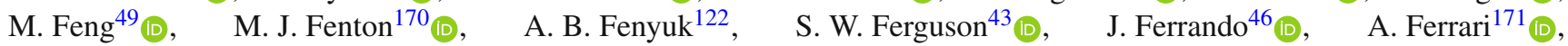

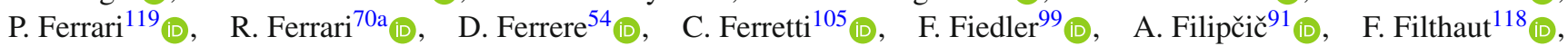

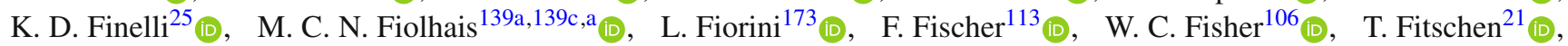

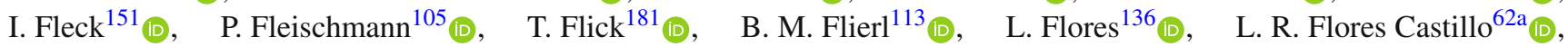

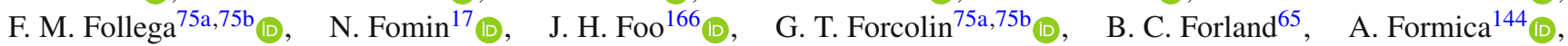

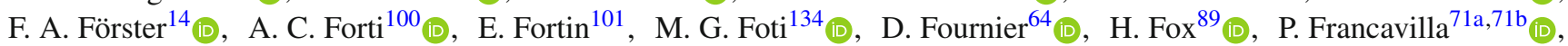

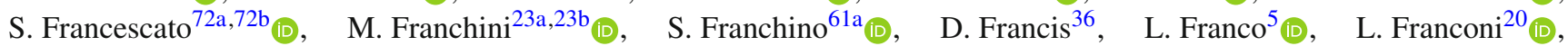

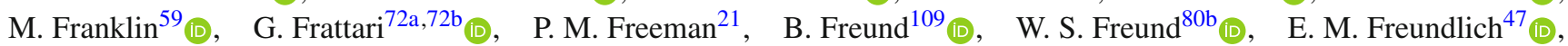
D. C. Frizzell ${ }^{128}$ (D), D. Froidevaux ${ }^{36}$ (D), J. A. Frost ${ }^{134}$ (D), M. Fujimoto ${ }^{126}$ (D), E. Fullana Torregrosa ${ }^{173}$ (D), T. Fusayasu ${ }^{115}$,

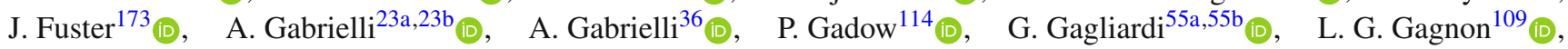

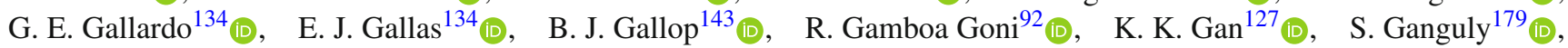

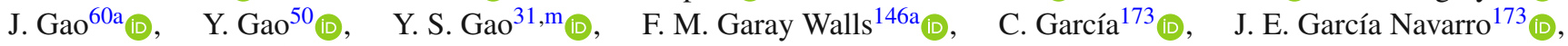
J. A. García Pascual ${ }^{15 a}{ }^{(0)}$, M. Garcia-Sciveres ${ }^{18}$ (D) R. W. Gardner ${ }^{37}$ (D) S. Gargiulo ${ }^{52}$ (D) C. A. Garner ${ }^{166}$, V. Garonne ${ }^{133}$ (D),

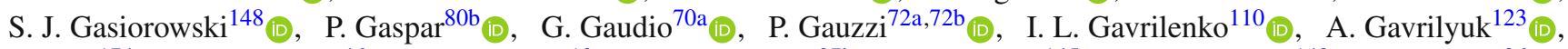
C. Gay ${ }^{174}$ (D), G. Gaycken ${ }^{46}$, E. N. Gazis ${ }^{10}$ (D), A. A. Geanta ${ }^{27 b}$ (D) C. M. Gee ${ }^{145}$ (D), C. N. P. Gee ${ }^{143}$ (D), J. Geisen ${ }^{96}$ (D), M. Geisen ${ }^{99}$ (D), C. Gemme ${ }^{55 b}$ (D), M. H. Genest ${ }^{58}$ (D), C. Geng ${ }^{105}$, S. Gentile ${ }^{72 a, 72 b}$ (D), S. George ${ }^{93}$ (D), T. Geralis ${ }^{44}$ (D), L. O. Gerlach ${ }^{53}$, P. Gessinger-Befurt ${ }^{99}$ (D) , G. Gessner ${ }^{47}$ (D) , M. Ghasemi Bostanabad ${ }^{175}$ (D), M. Ghneimat ${ }^{151}$ (D), A. Ghosh ${ }^{64}$ (D),

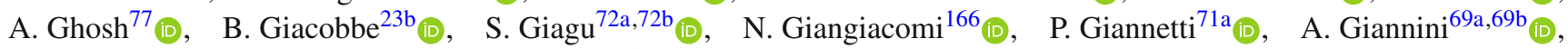

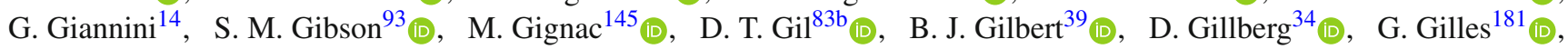

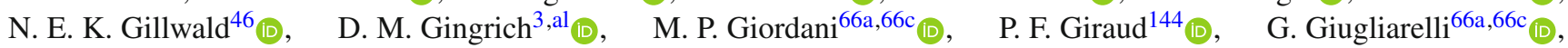

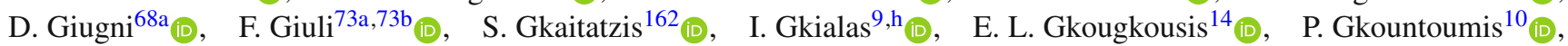

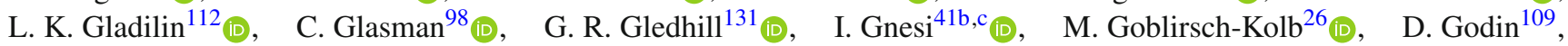
S. Goldfarb ${ }^{104}$ (D), T. Golling ${ }^{54}$ (D) D. Golubkov ${ }^{122}$ (D), A. Gomes ${ }^{139 a, 139 b}$ (D), R. Goncalves Gama ${ }^{53}$ (D) R. Ronçalo ${ }^{139 a, 139 c}{ }^{(D)}$, G. Gonella ${ }^{131}$ (D), L. Gonella ${ }^{21}$ (D), A. Gongadze ${ }^{79}$ (D), F. Gonnella ${ }^{21}$ (D), J. L. Gonski ${ }^{39}$ (D), S. González de la Hoz $^{173}$ (D), S. Gonzalez Fernandez ${ }^{14}$ (D), R. Gonzalez Lopez ${ }^{90}$ (D) $\quad$ C. Gonzalez Renteria ${ }^{18}$ (D), R. Gonzalez Suarez ${ }^{171}$ (D), S. Gonzalez-Sevilla ${ }^{54}$ (D), G. R. Gonzalvo Rodriguez ${ }^{173}$ (D) L. Goossens ${ }^{36}$ (D) N. A. Gorasia ${ }^{21}$ (D), P. A. Gorbounov ${ }^{123}$ (D),

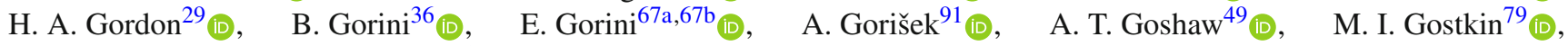

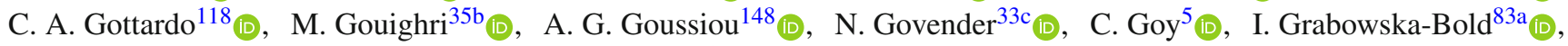

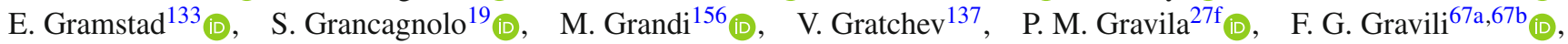

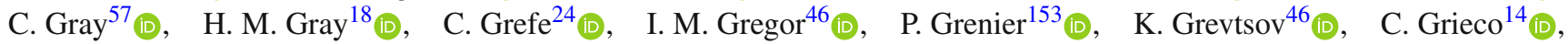
N. A. Grieser ${ }^{128}$, A. A. Grillo ${ }^{145}$, K. Grimm ${ }^{31,1}$ (D) S. Grinstein ${ }^{14, w}$ (D), J.-F. Grivaz ${ }^{64}$ (D), S. Groh ${ }^{99}$ (D), E. Gross ${ }^{179}$ (D),

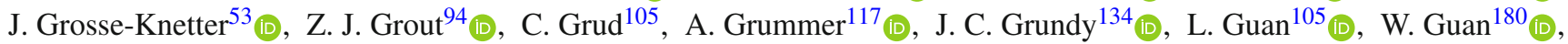
C. Gubbels ${ }^{174}$ (D), J. Guenther ${ }^{36}$ (D) , J. G. R. Guerrero Rojas ${ }^{173}$ (D), F. Guescini ${ }^{114}$ (D), D. Guest ${ }^{19,76}$ (D), R. Gugel ${ }^{99}$ (D),

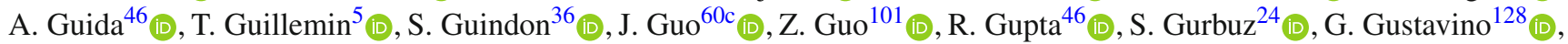
M. Guth ${ }^{52}$ (D), P. Gutierrez ${ }^{128}$ (D), L. F. Gutierrez Zagazeta ${ }^{136}$ (I) C. Gutschow ${ }^{94}$ (D) , C. Guyot ${ }^{144}$ (D), C. Gwenlan $^{134}$ (D),

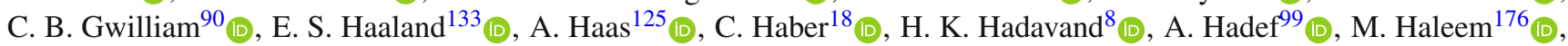

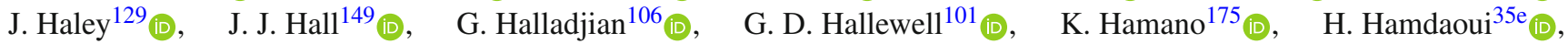




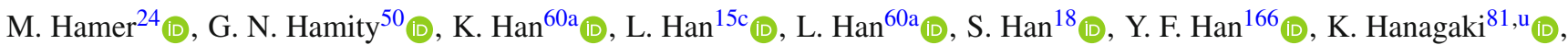

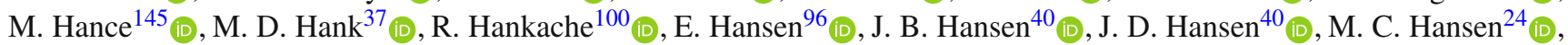

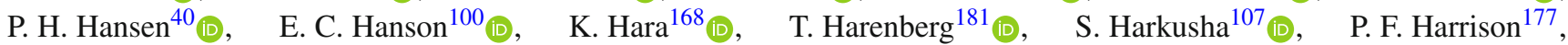

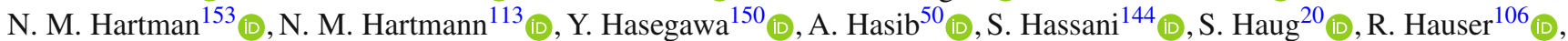
M. Havranek ${ }^{141}$ (D)， C. M. Hawkes ${ }^{21}$ (D)， R. J. Hawkings ${ }^{36}$ (D) S. Hayashida ${ }^{116}$ (D)， D. Hayden ${ }^{106}(\mathbb{D}), \quad$ C. Hayes ${ }^{105}$ (D),

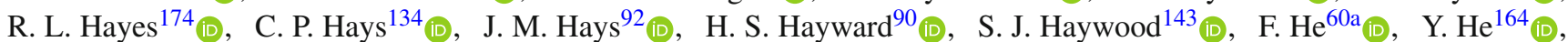
M. P. Heath ${ }^{50}$ (D) , V. Hedberg ${ }^{96}$ (D) A. L. Heggelund ${ }^{133}$ (D), N. D. Hehir ${ }^{92}$ (D) , C. Heidegger ${ }^{52}$ (D) , K. K. Heidegger $^{52}$ (D),

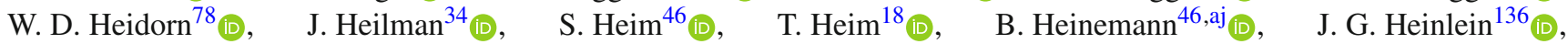

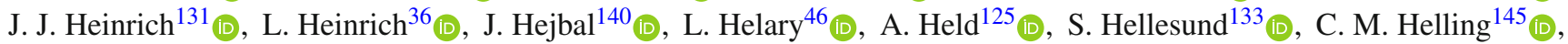

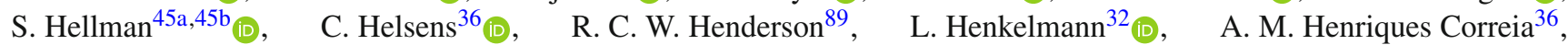

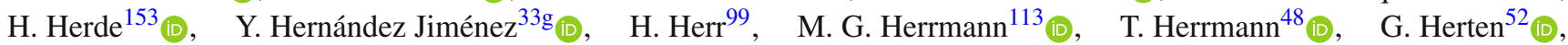

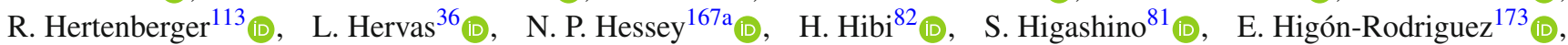

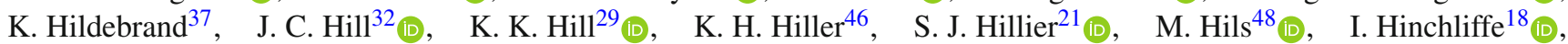

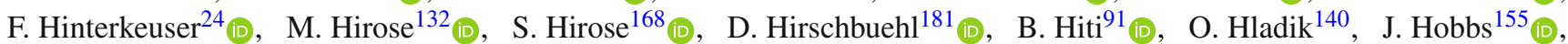

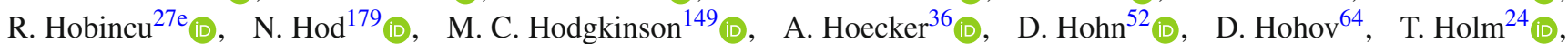
T. R. Holmes ${ }^{37}$ (D), M. Holzbock ${ }^{114}$ (D), L. B. A. H. Hommels ${ }^{32}$ (D), T. M. Hong ${ }^{138}$ (D), J. C. Honig ${ }^{52}$ (D), A. Hönle ${ }^{114}$ (D), B. H. Hooberman ${ }^{172}$ (D), W. H. Hopkins ${ }^{6}$ (D), Y. $\operatorname{Horii}^{116}$ (D), P. $\operatorname{Horn}^{48}$ (D), L. A. $\operatorname{Horyn}^{37}$ (D), S. Hou ${ }^{158}$ (D), J. Howarth ${ }^{57}$ (D),

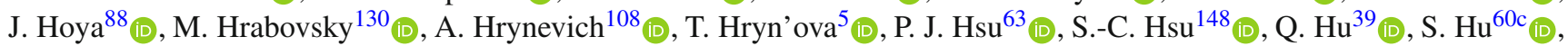

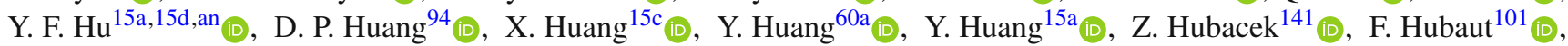

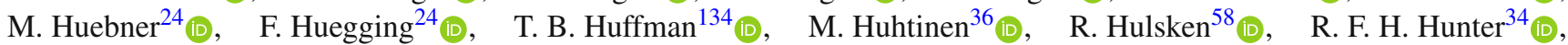

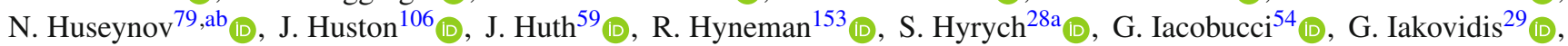
I. Ibragimov ${ }^{151}$ (D), L. Iconomidou-Fayard ${ }^{64}$ (D), P. Iengo ${ }^{36}$ (D) R. Ignazzi $^{40}$, R. Iguchi $^{163}$ (D), T. Iizawa ${ }^{54}$ (D), Y. Ikegami ${ }^{81}$ (D),

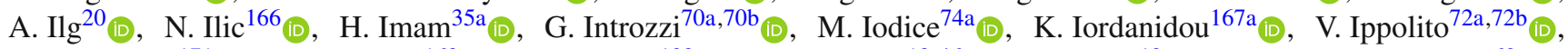

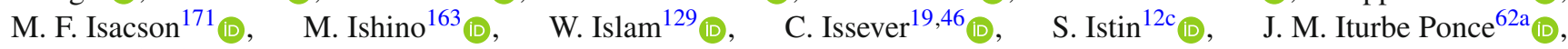

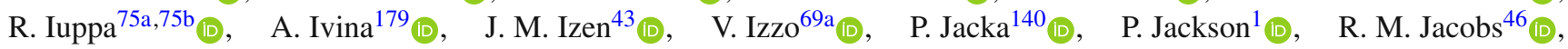
B. P. Jaeger ${ }^{152}$ (D), G. Jäkel ${ }^{181}$ (D), K. B. Jakobi ${ }^{99}$, K. Jakobs ${ }^{52}$ (D), T. Jakoubek ${ }^{179}$ (D), J. Jamieson ${ }^{57}$ (D), K. W. Janas ${ }^{83 a}$ (D),

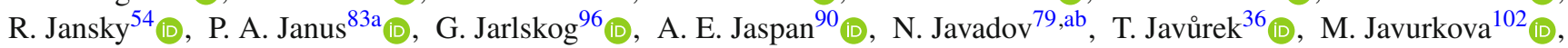

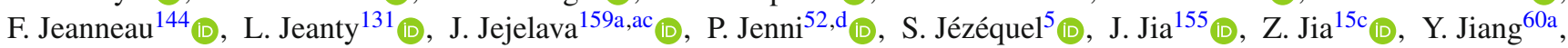

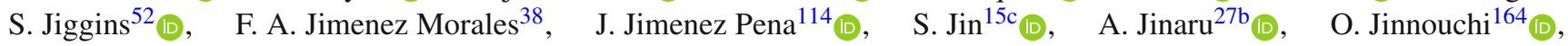

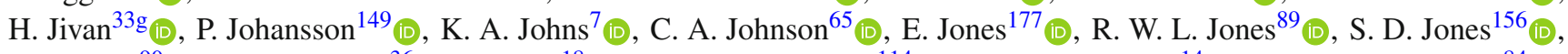

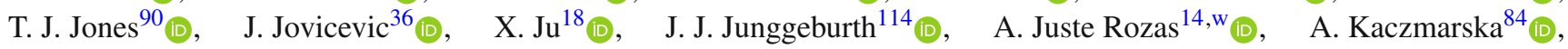

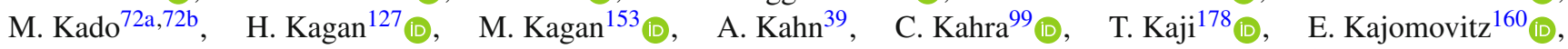
C. W. Kalderon ${ }^{29}$ (D), A. Kaluza ${ }^{99}, \quad$ A. Kamenshchikov ${ }^{122}$ (D), M. Kaneda ${ }^{163}$ (D), N. J. Kang ${ }^{145}$ (I), S. Kang ${ }^{78}$ (D),

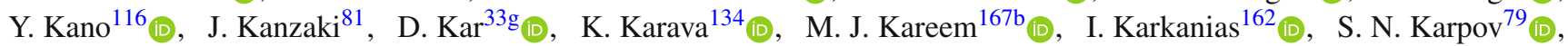

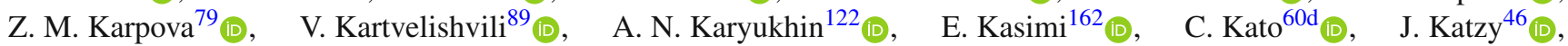
K. Kawade ${ }^{150}$ (D) K. Kawagoe ${ }^{87}$ (D), T. Kawaguchi ${ }^{116}$ (D) T. Kawamoto ${ }^{144}$ (D) , G. Kawamura ${ }^{53}$, E. F. Kay ${ }^{175}$ (D), F. I. Kaya ${ }^{169}$ (D),

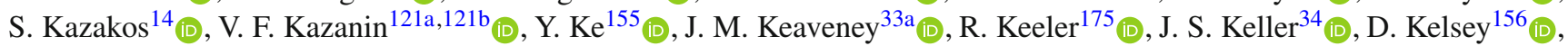

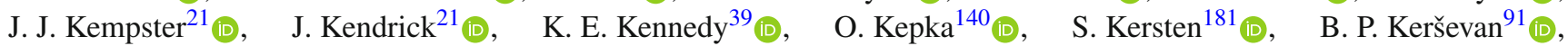
S. Ketabchi Haghighat ${ }^{166}$ (D), F. Khalil-Zada ${ }^{13}, \quad$ M. Khandoga ${ }^{144}$ (D), A. Khanov ${ }^{129}$ (D), A. G. Kharlamov ${ }^{121 a, 121 b}{ }_{(\text {D }}$,

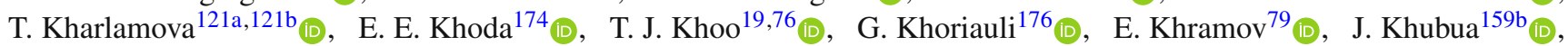

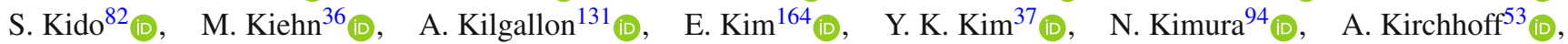

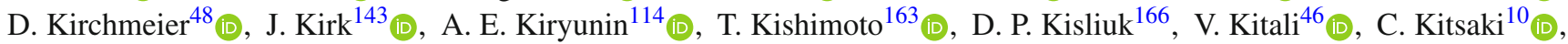

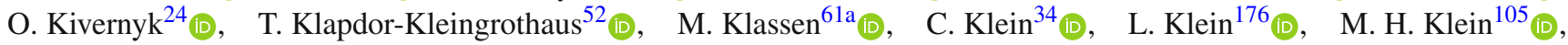

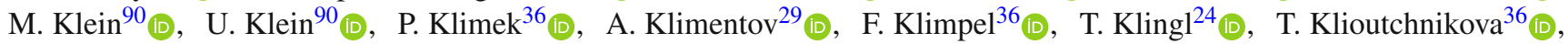

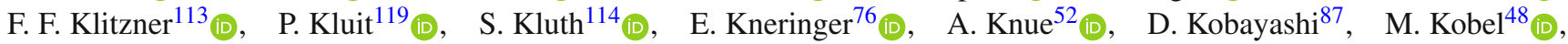

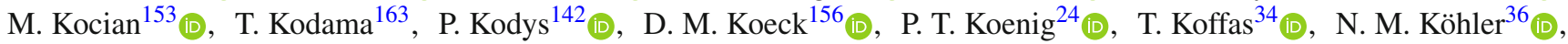

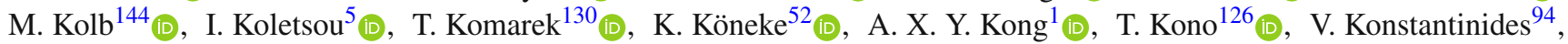

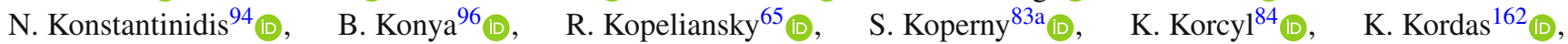
G. Koren ${ }^{161}$, A. Korn ${ }^{94}$ (D), I. Korolkov ${ }^{14}$ (D), E. V. Korolkova ${ }^{149}$, N. Korotkova ${ }^{112}$ (D), O. Kortner ${ }^{14}$ (D), S. Kortner ${ }^{114}$ (D), V. V. Kostyukhin ${ }^{149,165}$ (D) A. Kotsokechagia ${ }^{64}$ (D) , A. Kotwal $^{49}$ (D), A. Koulouris ${ }^{10}$ (D), A. Kourkoumeli-Charalampidi ${ }^{70 a}, 70 b$ (D), C. Kourkoumelis ${ }^{9}$ (D) , E. Kourlitis ${ }^{6}$ (D), R. Kowalewski ${ }^{175}$ (D), W. Kozanecki ${ }^{144}$ (D) A. S. Kozhin ${ }^{122}$ (D), V. A. Kramarenko ${ }^{112}$ (D),

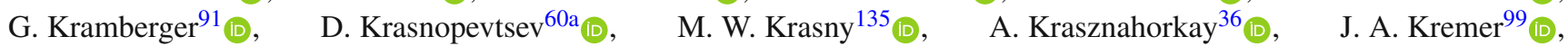

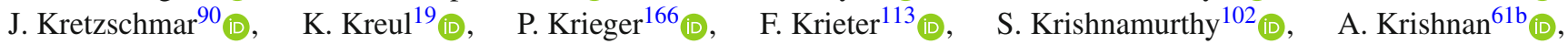




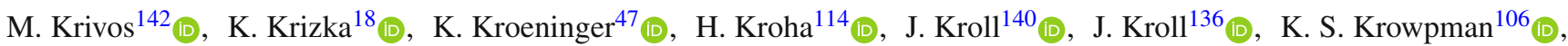

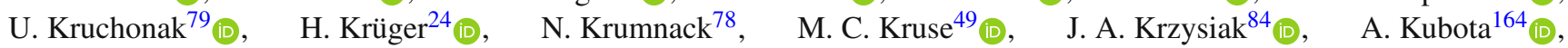
O. Kuchinskaia ${ }^{165}$ (D), S. Kuday ${ }^{4 b}$ (D), D. Kuechler ${ }^{46}$ (D), J. T. Kuechler ${ }^{46}$ (D), S. Kuehn ${ }^{36}$ (D), T. Kuhl ${ }^{46}$ (D), V. Kukhtin ${ }^{79}$ (D),

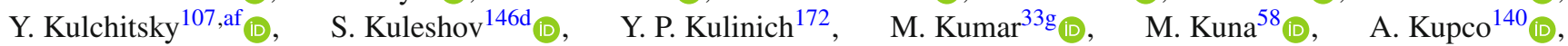

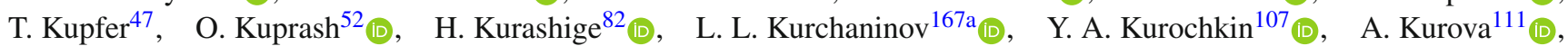

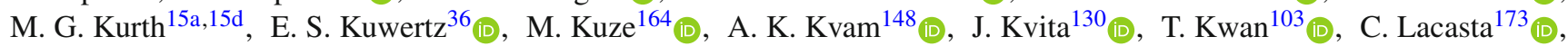

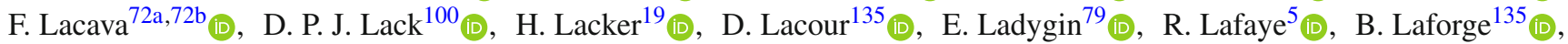

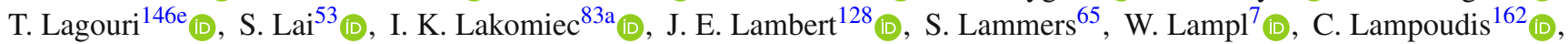
E. Lançon ${ }^{29}$ (D), U. Landgraf ${ }^{52}$ (D), M. P. J. Landon ${ }^{92}$ (D), V. S. Lang ${ }^{52}$ (D), J. C. Lange ${ }^{53}$ (D), R. J. Langenberg ${ }^{102}$ (D),

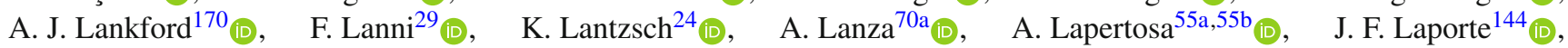

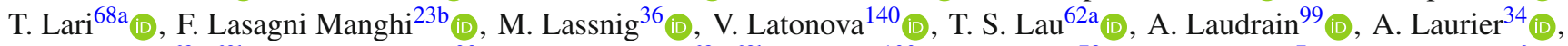

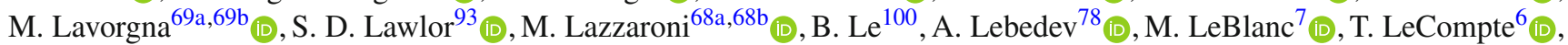

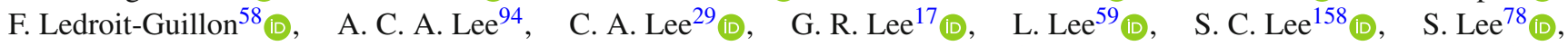

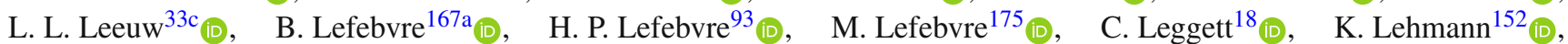

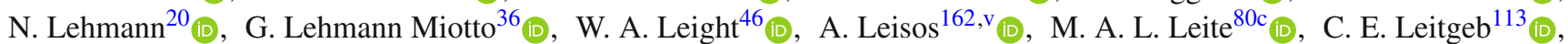

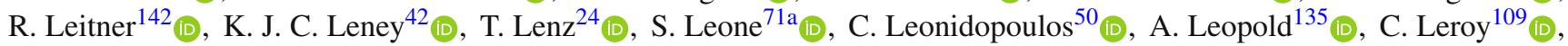
R. Les ${ }^{106}(\mathbb{D})$ C. G. Lester $^{32}$ (D), M. Levchenko ${ }^{137}$ (D), J. Levêque ${ }^{5}$, D. Levin ${ }^{105}$ (D), L. J. Levinson ${ }^{179}$ (D), D. J. Lewis ${ }^{21}$ (D),

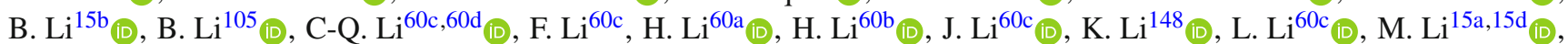

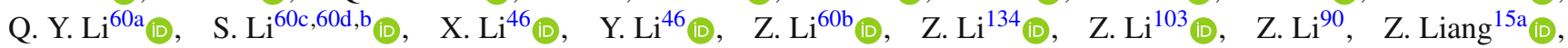

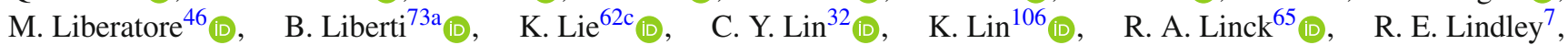

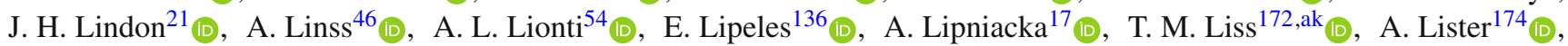

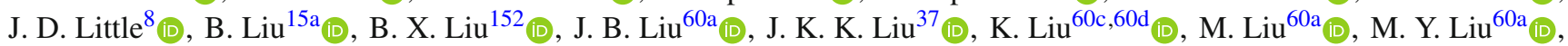

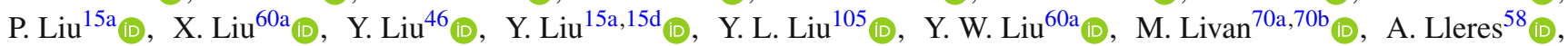
J. Llorente Merino ${ }^{152}$ (I), S. L. Lloyd ${ }^{92}$ (D), E. M. Lobodzinska ${ }^{46}$ (D), P. Loch $^{7}$ (D), S. Loffredo ${ }^{73 a}, 73 b$ (D), T. Lohse ${ }^{19}$ (D),

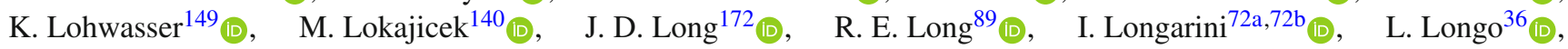
R. Longo ${ }^{172}$ (D), I. Lopez Paz ${ }^{100}$, A. Lopez Solis ${ }^{149}$ (D), J. Lorenz ${ }^{113}$ (D), N. Lorenzo Martinez ${ }^{5}$ (D) A. M. Lory ${ }^{113}$ (D),

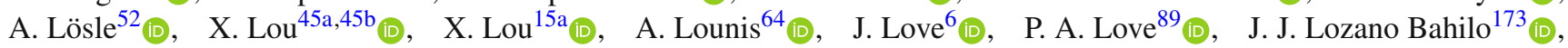

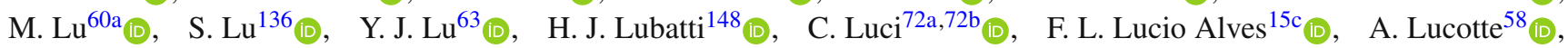

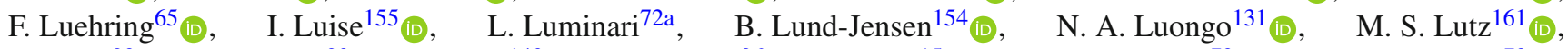

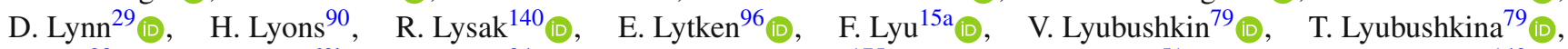

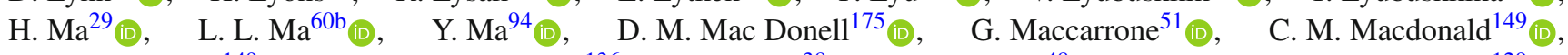
J. C. MacDonald ${ }^{149}$ (D), J. Machado Miguens ${ }^{136}$ (D), R. $\operatorname{Madar}^{38}$ (D), W. F. $\operatorname{Mader}^{48}$ (D), M. Madugoda Ralalage $\operatorname{Don}^{129}$ (D), N. Madysa ${ }^{48}$ (D), J. Maeda ${ }^{82}$ (D), T. Maeno ${ }^{29}$ (D), M. Maerker ${ }^{48}$ (D), V. Magerl ${ }^{52}$ (I), J. Magro ${ }^{66 a, 66 c, r}$ (D), D. J. Mahon ${ }^{39}$ (D),

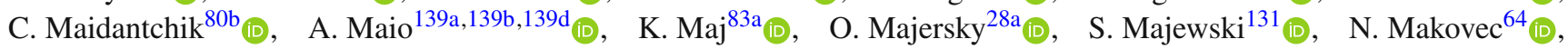

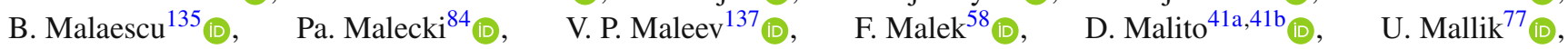
C. Malone $^{32}$ (D), S. Maltezos $^{10}$, S. Malyukov ${ }^{79}$, J. Mamuzic ${ }^{173}$ (D), G. Mancini ${ }^{51}$ (D), J. P. Mandalia ${ }^{92}$ (D), I. Mandić ${ }^{91}$ (D), L. Manhaes de Andrade Filho ${ }^{80}{ }_{(\mathbb{D}}$, I. M. Maniatis ${ }^{162}$ (D), J. Manjarres Ramos ${ }^{48}$ (D) K. H. Mankinen ${ }^{96}$ (D), A. Mann ${ }^{113}$ (D),

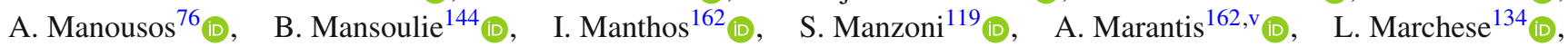

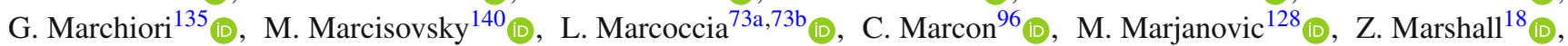

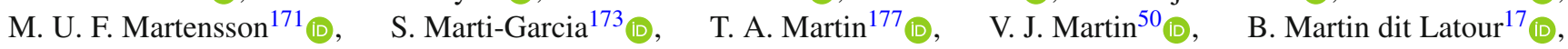
L. Martinelli ${ }^{74 a, 74 b} \mathbb{D}_{\text {D }}$, M. Martinez ${ }^{14, w}$ (D) P. Martinez Agullo ${ }^{173}$ (D) V. I. Martinez Outschoorn ${ }^{102}$ (D) S. Martin-Haugh ${ }^{143}$ (D), V. S. Martoiu ${ }^{27 b}$ (D), A. C. $\operatorname{Martyniuk}^{94}$ (D), A. $\operatorname{Marzin}^{36}$ (D), S. R. Maschek ${ }^{114}$ (D), L. Masetti ${ }^{99}$ (D), T. Mashimo ${ }^{163}$ (D),

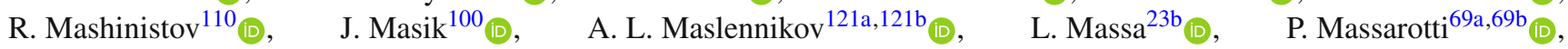

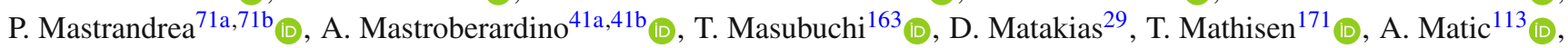

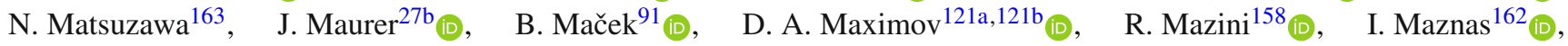
S. M. $\operatorname{Mazza}^{145}$ (D) C. Mc Ginn ${ }^{29}$ (D) J. P. Mc Gowan ${ }^{103}$ (D) , S. P. Mc Kee ${ }^{105}$ (D), T. G. McCarthy ${ }^{114}$ (D), W. P. McCormack ${ }^{18}$ (D),

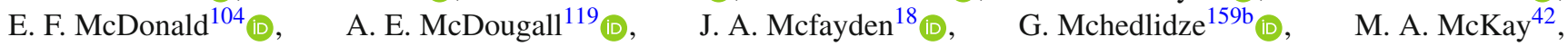

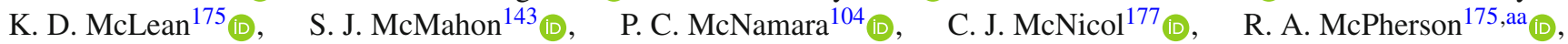

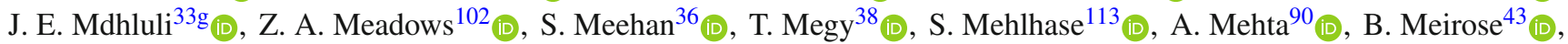
D. Melini ${ }^{160}\left(\mathbb{D}, \quad\right.$ B. R. Mellado Garcia ${ }^{33 g_{(D)}, \quad \text { F. Meloni }}{ }^{46}, \quad$ A. Melzer ${ }^{24}$ (D), E. D. Mendes Gouveia ${ }^{139 a, 139 e} e_{\mathbb{D}}$,

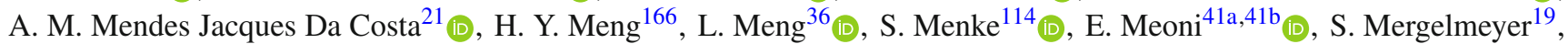

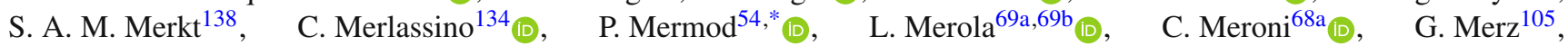

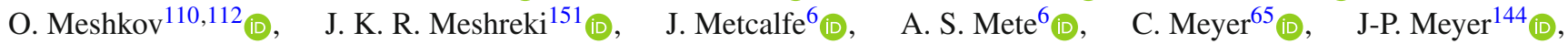




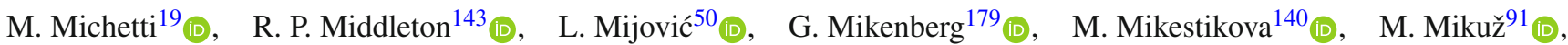

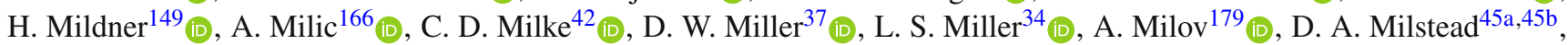
A. A. Minaenko ${ }^{122}$ (D), I. A. Minashvili ${ }^{159 b}$ (D), L. Mince $^{57}$ (D), A. I. $\operatorname{Mincer}^{125}$ (D), B. Mindur ${ }^{83 \mathrm{a}}$ (D), M. Mineev ${ }^{79}$ (D), Y. Minegishi ${ }^{163}$, Y. Mino ${ }^{85}$ (D), L. M. Mir $^{14}$ (D), M. Mironova ${ }^{134}$ (D), T. Mitani ${ }^{178}$ (D), J. Mitrevski ${ }^{113}$, V. A. Mitsou ${ }^{173}$ (D),

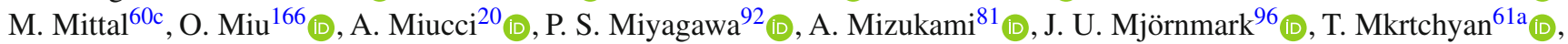

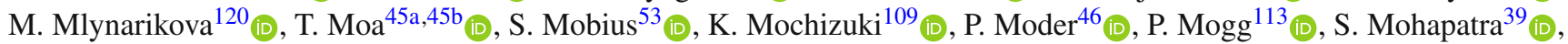

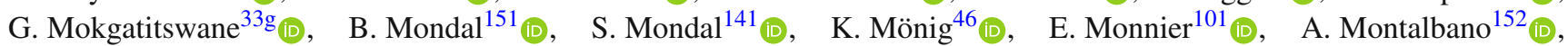

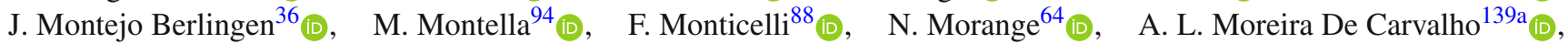
M. Moreno Llácer ${ }^{173}$ (i), C. Moreno Martinez ${ }^{14}$ (i), P. Morettini $^{55 b}$ (i),$\quad$ M. Morgenstern ${ }^{160}$ (I),$\quad$ S. Morgenstern ${ }^{177}$ (D),

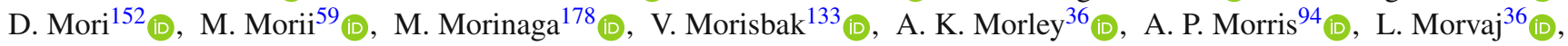

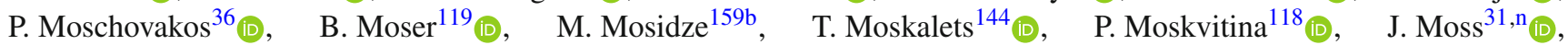
E. J. W. Moyse ${ }^{102}$ (D), S. Muanza ${ }^{101}$ (D), J. Mueller ${ }^{138}$ (D), D. Muenstermann ${ }^{89}$ (D), G. A. Mullier ${ }^{96}$ (D), J. J. Mullin ${ }^{136}$,

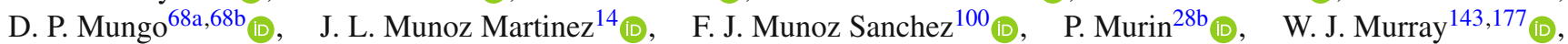

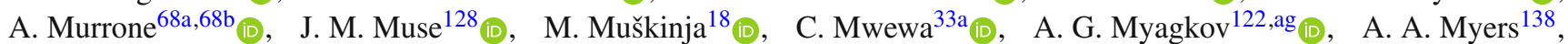
G. Myers ${ }^{65}$ (D), J. Myers ${ }^{131}$ (D), M. Myska ${ }^{141}$ (D), B. P. Nachman ${ }^{18}$ (D), O. Nackenhorst ${ }^{47}$ (D), A.Nag Nag ${ }^{48}$ (D), K. Nagai ${ }^{134}$ (D), K. Nagano ${ }^{81}$ (D) J. L. Nagle ${ }^{29}$ (D), E. Nagy ${ }^{101}$ (D), A. M. Nairz ${ }^{36}$ (D) Y. Nakahama ${ }^{116}$ (D), K. Nakamura ${ }^{81}$ (D) H. Nanjo ${ }^{132}$ (D), F. Napolitano ${ }^{61 \mathrm{a}}$ (D), R. F. Naranjo Garcia ${ }^{46}$ (D), R. Narayan ${ }^{42}$ (D), I. Naryshkin ${ }^{137}$ (D), M. Naseri ${ }^{34}$ (D), T. Naumann ${ }^{46}$ (D),

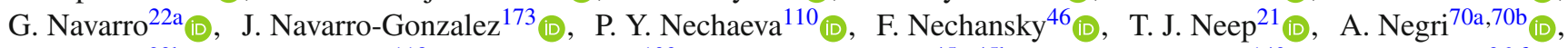

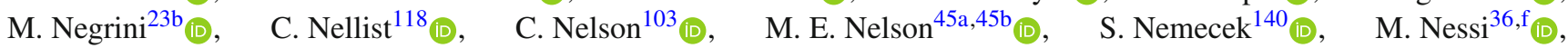

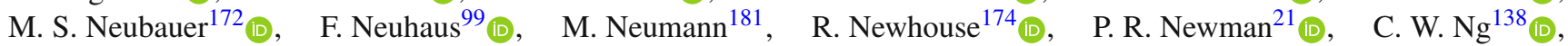

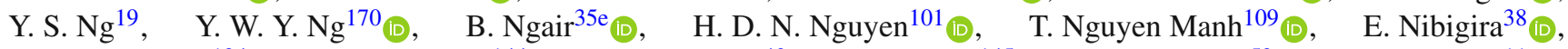

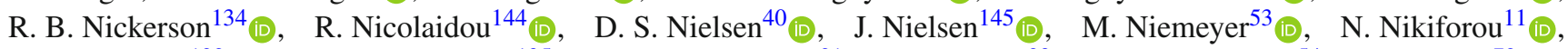

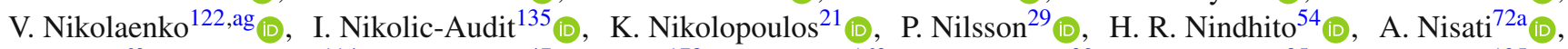
N. Nishu ${ }^{60}$ (D) , R. Nisius ${ }^{114}$ (D) I. Nitsche ${ }^{47}$, T. Nitta ${ }^{178}$ (D) T. Nobe ${ }^{163}$ (D), D. L. Noel ${ }^{32}$ (D), Y. Noguchi ${ }^{85}$ (D) I. Nomidis ${ }^{135}$ (D),

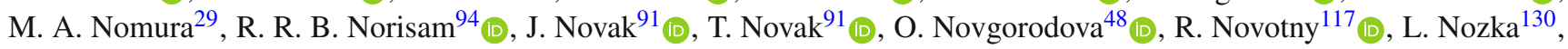
K. Ntekas ${ }^{170}$ (D) E. Nurse ${ }^{94}$, F. G. Oakham ${ }^{34, a 1}$ (D) J. Ocariz ${ }^{135}$ (D), A. Ochi ${ }^{82}$ (D), I. Ochoa ${ }^{139 a}$ (D), J. P. Ochoa-Ricoux ${ }^{146 a}$ (D),

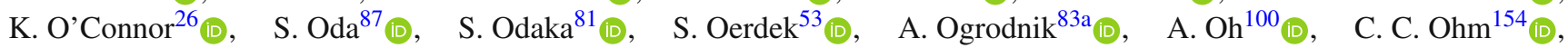

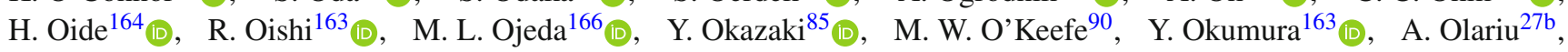

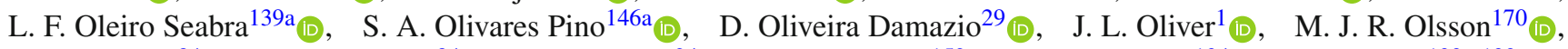
A. Olszewski ${ }^{84}$ (D, J. Olszowska ${ }^{84}$ (D), Ö.O. Öncel ${ }^{24}$ (D), D. C. O'Neil ${ }^{152}$ (D), A. P. O'neill ${ }^{134}$ (D), A. Onofre ${ }^{139 a, 139 e} \mathrm{e}_{\mathbb{D}}$,

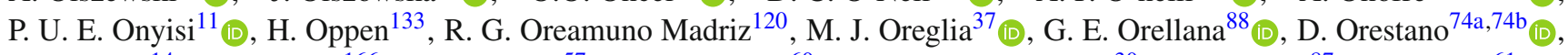

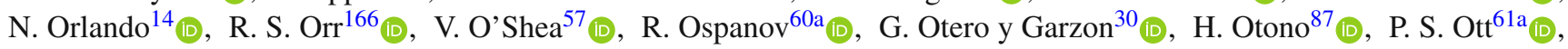

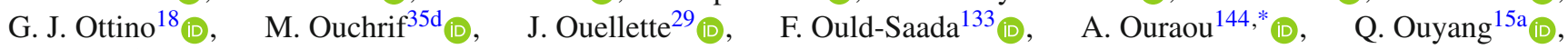
M. Owen ${ }^{57}$ (1), R. E. Owen ${ }^{143}$ (D), V. E. Ozcan ${ }^{12 c}$ (I), N. Ozturk ${ }^{8}$ (D), J. Pacalt ${ }^{130}{ }^{6}$, H. A. Pacey ${ }^{32}$ (D), K. Pachal ${ }^{49}$ (D),

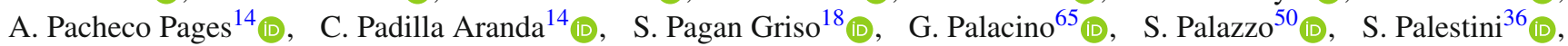

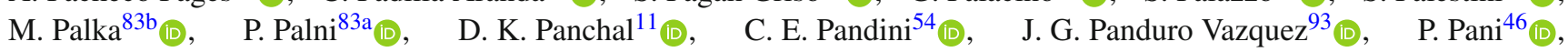
G. Panizzo ${ }^{66 a, 66 c}$ (D), L. Paolozzi ${ }^{54}$ (D) C. Papadatos ${ }^{109}$ (D), S. Parajuli ${ }^{42}$ (D), A. Paramonov ${ }^{6}$ (D) C. Paraskevopoulos ${ }^{10}$ (D),

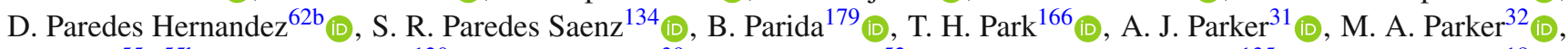
F. Parodi ${ }^{55 a, 55 b}$ (D) E. W. Parrish ${ }^{120}$ (I) J. A. Parsons ${ }^{39}$ (D), U. Parzefall ${ }^{52}$ (D) L. Pascual Dominguez ${ }^{135}$ (D) V. R. Pascuzzi ${ }^{18}$ (D),

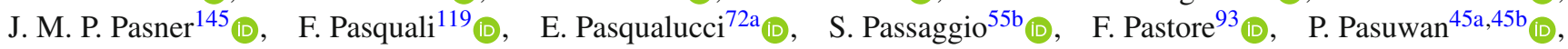

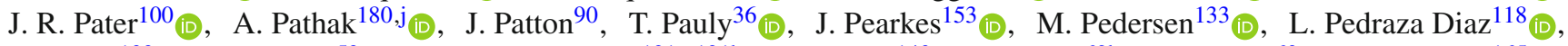

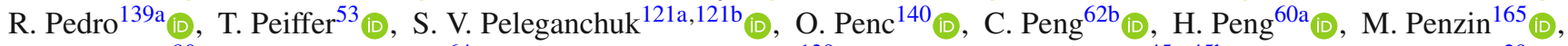

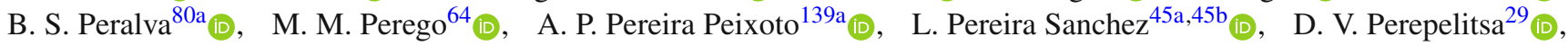

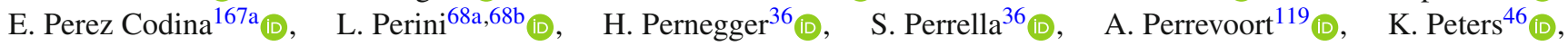

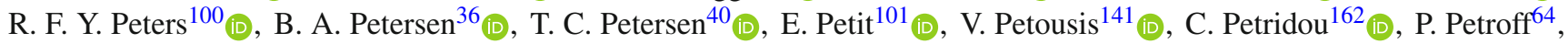

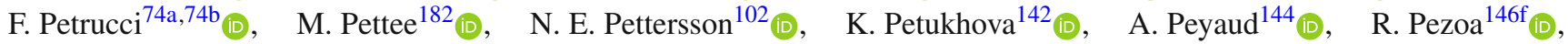

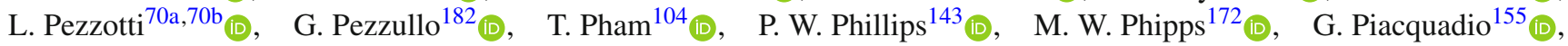

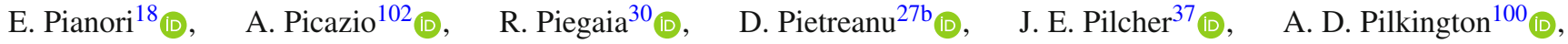
M. Pinamonti ${ }^{66 a, 66 c}$ (D), J. L. Pinfold ${ }^{3}$ (D), C. Pitman Donaldson ${ }^{94}$, L. Pizzimento ${ }^{73 a}, 73 b$ (D), A. Pizzini ${ }^{119}$ (D),

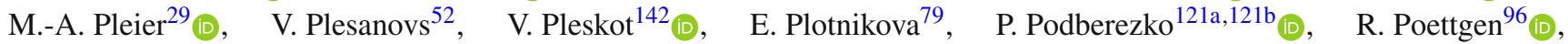

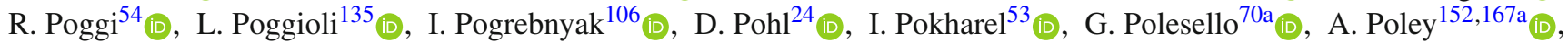

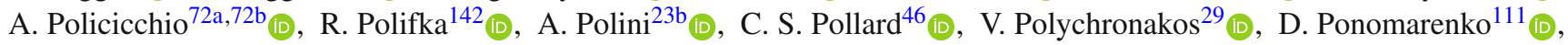

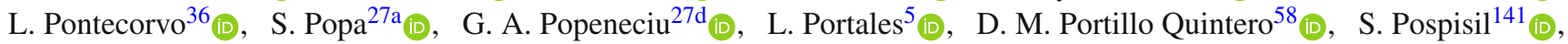




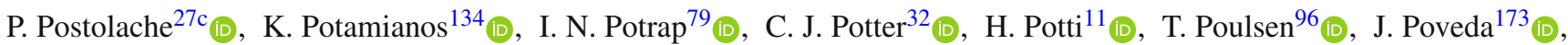

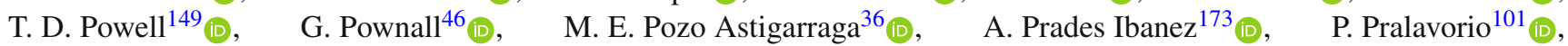

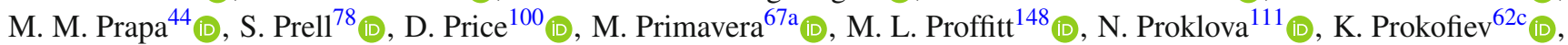

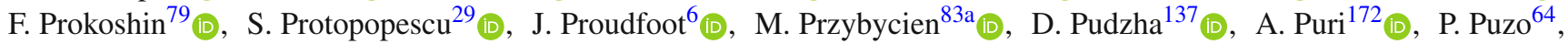

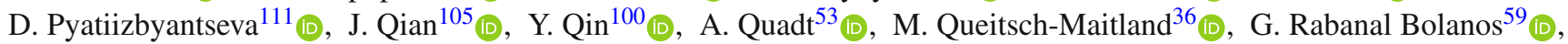

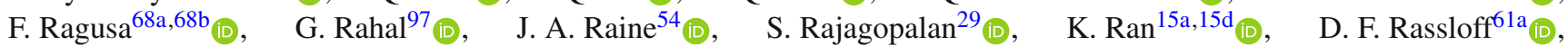

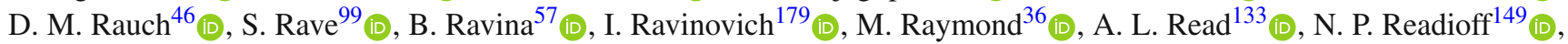
M. Reale ${ }^{67 a, 67 b}$ (D), D. M. Rebuzzi ${ }^{70 a, 70 b}$ (I) G. Redlinger ${ }^{29}$ (D), K. Reeves ${ }^{43}$ (D), D. Reikher ${ }^{161}$ (D), A. Reiss ${ }^{99}$, A. Rej $^{151}$ (D),

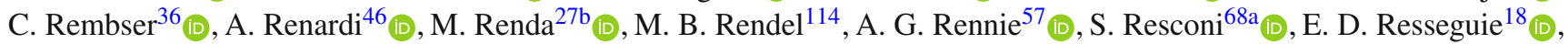
S. Rettie ${ }^{94}$ (I) $\quad$ B. Reynolds ${ }^{127}$, E. Reynolds ${ }^{21}$ (D), O. L. Rezanova ${ }^{121 a, 121 b}$ (i), P. Reznicek ${ }^{142}$ (I), E. Ricci ${ }^{75 a, 75 b}$ (D),

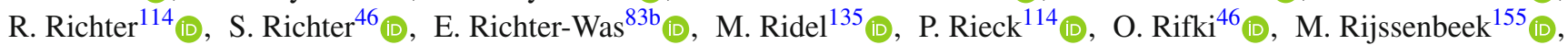
A. Rimoldi ${ }^{70 a, 70 b}$ (D) , M. Rimoldi ${ }^{46}$ (D) L. Rinaldi ${ }^{23 a, 23 b}$ (D) T. T. Rinn ${ }^{172}$ (D), G. Ripellino ${ }^{154}$ (D), I. Riu ${ }^{14}$ (D) , P. Rivadeneira ${ }^{46}$ (D),

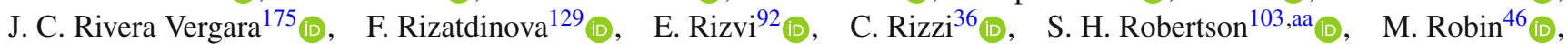

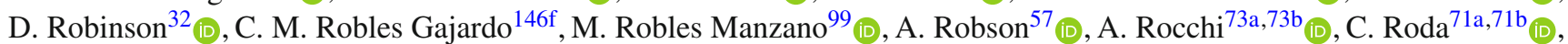

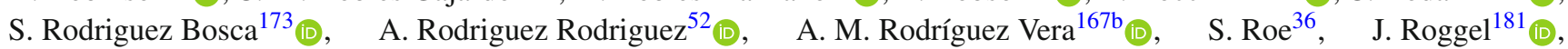

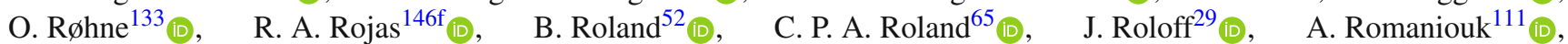

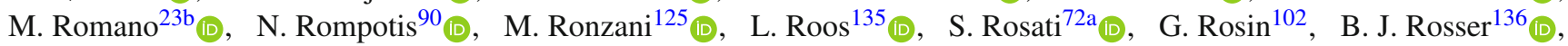

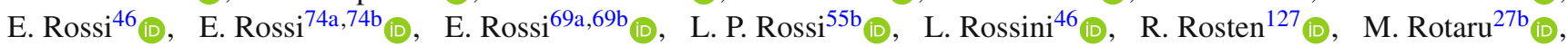

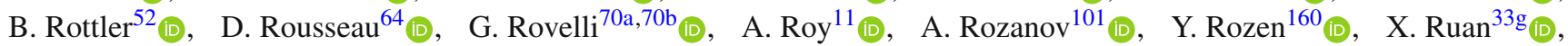

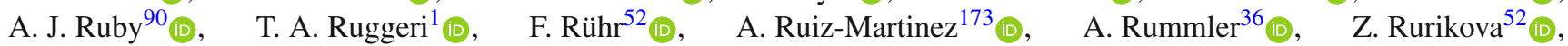
N. A. Rusakovich ${ }^{79}$ (D) H. L. Russell ${ }^{103}$ (D) L. Rustige ${ }^{38,47}$ (D) J. P. Rutherfoord ${ }^{7}$, E. M. Rüttinger ${ }^{149}$ (D), M. Rybar ${ }^{142}$ (D),

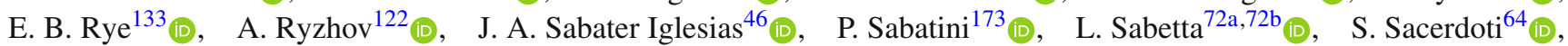

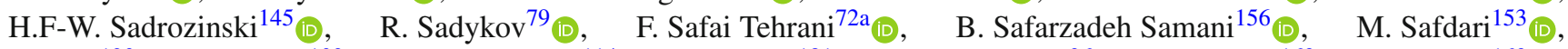

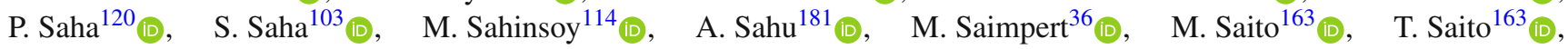
D. Salamani ${ }^{54}$, G. Salamanna ${ }^{74 a, 74 b}{ }_{(\mathbb{D})}$, A. Salnikov ${ }^{153}$ (D), J. Salt ${ }^{173}$ (D), A. Salvador Salas ${ }^{14}$ (D), D. Salvatore ${ }^{41 a, 41 b}$ (D), $^{1}$, F. Salvatore ${ }^{156}$ (D) A. Salzburger ${ }^{36}$ (D) D. Sammel ${ }^{52}$ (i), D. Sampsonidis ${ }^{162}$ (i), D. Sampsonidou ${ }^{60 c, 60 d}$ (I) J. Sánchez ${ }^{173}$ (D),

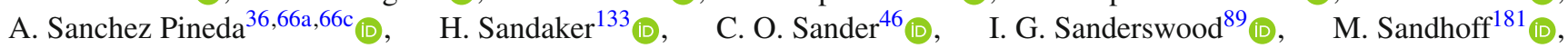
$\begin{array}{lll}\text { C. Sandoval } & 22 \mathrm{~b} \\ \mathbb{1}\end{array}$

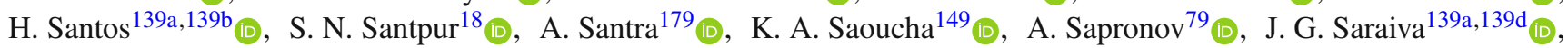
J. Sardain ${ }^{135}$ (D), O. Sasaki ${ }^{81}$ (D), K. Sato ${ }^{168}$ (D), F. Sauerburger ${ }^{52}$ (D) E. Sauvan ${ }^{5}$ (D), P. Savard ${ }^{166, a 1}$ (D), R. Sawada ${ }^{163}$ (D), C. Sawyer ${ }^{143}$ (D), L. Sawyer ${ }^{95}$ (D), I. Sayago Galvan ${ }^{173}, \quad$ C. Sbarra ${ }^{23 b}$ (D), A. Sbrizzi ${ }^{66 a, 66 c}$ (D), T. Scanlon ${ }^{94}$ (D), J. Schaarschmidt ${ }^{148}$ (D), P. Schacht ${ }^{114}$ (D), D. Schaefer ${ }^{37}$ (D), L. Schaefer ${ }^{136}{ }^{(1)}, \quad$ U. Schäfer ${ }^{99}$ (D), A. C. Schaffer ${ }^{64}$ (D),

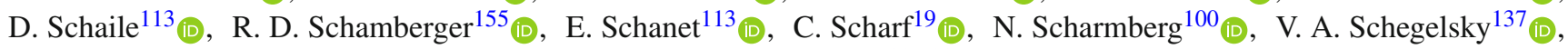

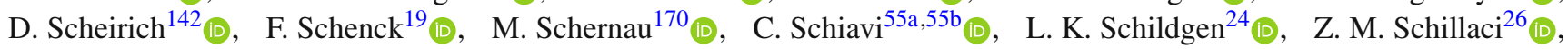

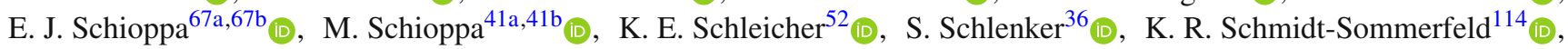

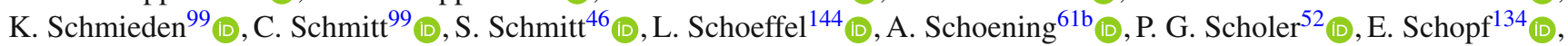
M. Schott ${ }^{99}$ (D), J. F. P. Schouwenberg ${ }^{118}$ (D), J. Schovancova ${ }^{36}$ (D) S. Schramm ${ }^{54}$ (D), F. Schroeder ${ }^{181}$ (D), A. Schulte ${ }^{99}{ }^{(1)}$,

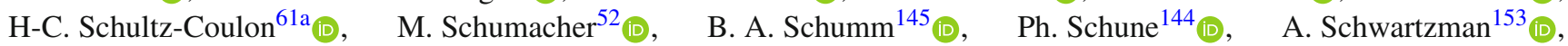

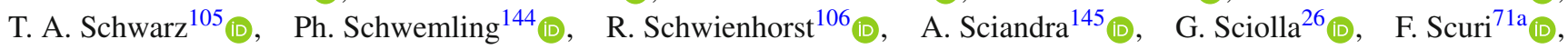

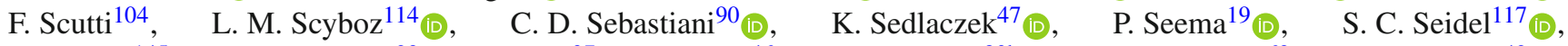

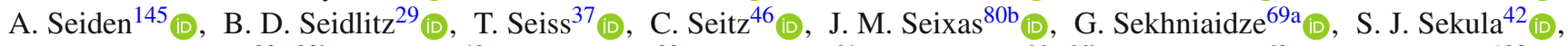

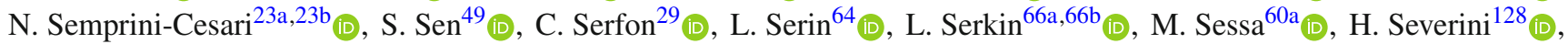

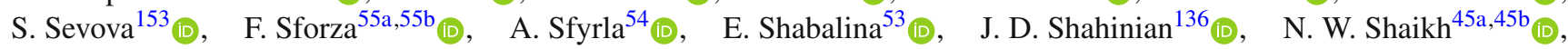

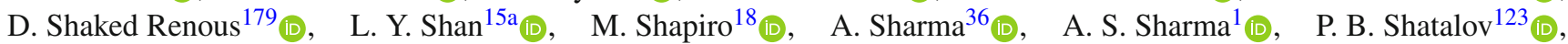

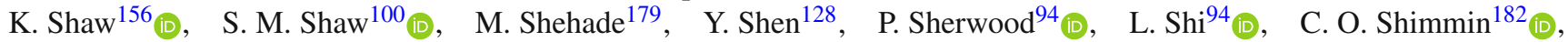

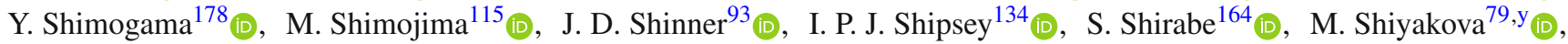
J. Shlomi ${ }^{179}$ (D), M. J. Shochet ${ }^{37}$ (D) J. Shojaii ${ }^{104}$ (D), D. R. Shope ${ }^{154}$ (D), S. Shrestha ${ }^{127}$ (D), E. M. Shrif ${ }^{339}$ (I) , M. J. Shroff ${ }^{175}$ (D),

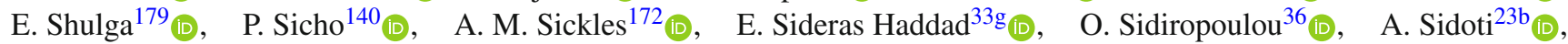
F. Siegert ${ }^{48}$ (D), Dj. Sijacki ${ }^{16}$ (D), M. V. Silva Oliveira ${ }^{36}$ (D), S. B. Silverstein ${ }^{45 a}$ (D), S. Simion ${ }^{64}$, R. Simoniello ${ }^{99}$ (D),

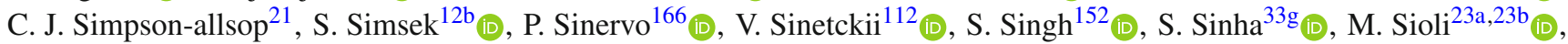
I. Siral ${ }^{131}$ (D), S. Yu. Sivoklokov ${ }^{112}$ (D), J. Sjölin ${ }^{45 a, 45 b}$ (D), A. Skaf ${ }^{53}$ (D), E. Skorda ${ }^{96}$ (D), P. Skubic ${ }^{128}$ (D), M. Slawinska ${ }^{84}$ (D),

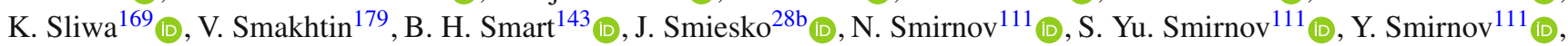

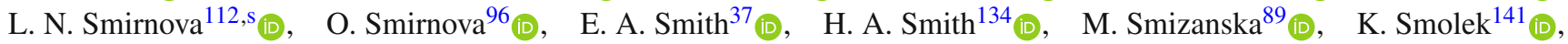




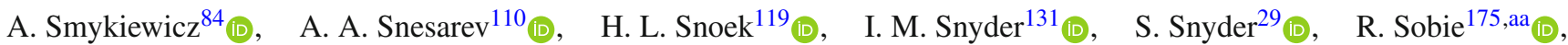
A. Soffer ${ }^{161}$ (D), A. Søgaard $^{50}$ (D), F. Sohns ${ }^{53}$ (D), C. A. Solans Sanchez ${ }^{36}$ (D), E. Yu. Soldatov ${ }^{111}$ (D), U. Soldevila ${ }^{173}$ (D), A. A. Solodkov ${ }^{122}$ (i), A. Soloshenko ${ }^{79}$ (i) , O. V. Solovyanov ${ }^{122}$ (D), V. Solovyev ${ }^{137}$ (D), P. Sommer ${ }^{149}$ (D), H. Son ${ }^{169}$ (D),

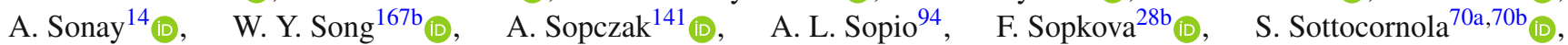

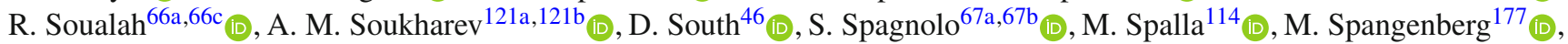

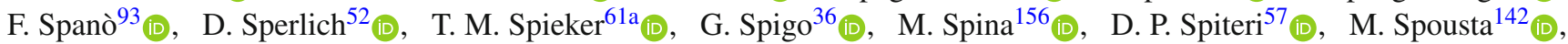

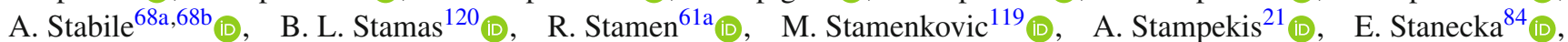

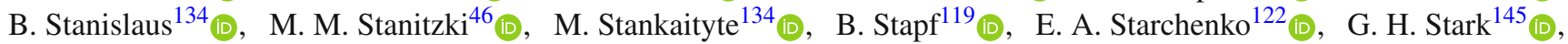

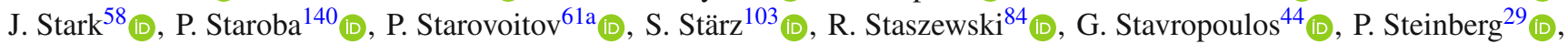

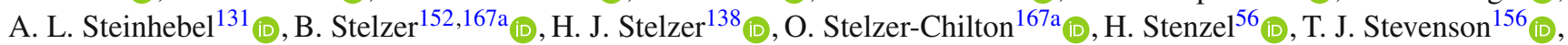

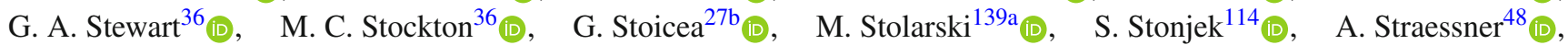
J. Strandberg ${ }^{154}$ (D), S. Strandberg ${ }^{45 a, 45 b}$ (D),$\quad$ M. Strauss ${ }^{128}$ (D), T. Strebler ${ }^{101}$ (D), P. Strizenec ${ }^{28 b} b_{(\mathbb{D})}, \quad$ R. Ströhmer ${ }^{176}$ (D),

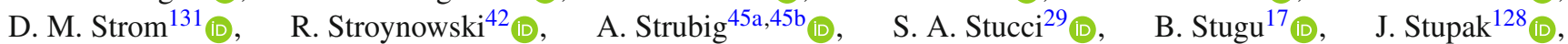

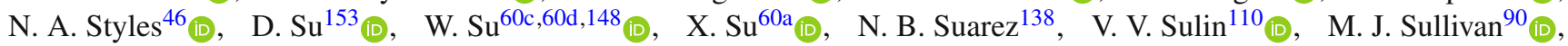

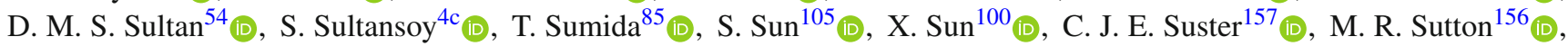

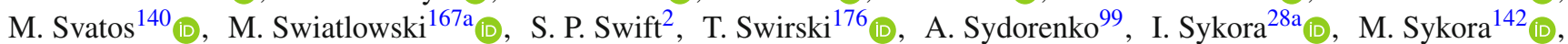

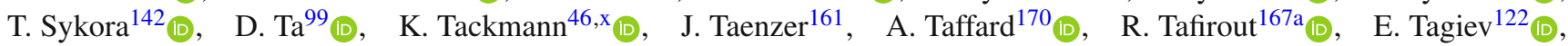

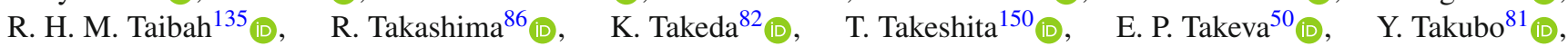

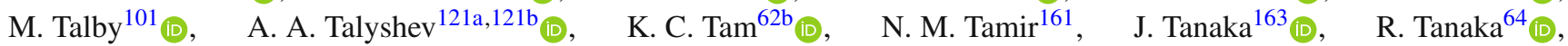

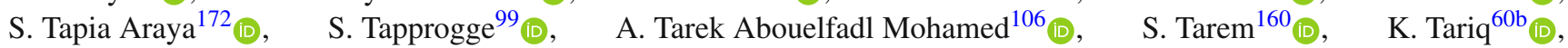

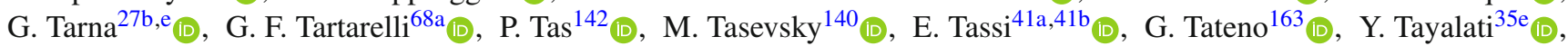
G. N. Taylor ${ }^{104}$ (I),$\quad$ W. Taylor ${ }^{167 b}$ (D), H. Teagle ${ }^{90}, \quad$ A. S. Tee ${ }^{89}$ (D), R. Teixeira De Lima ${ }^{153}$ (D), P. Teixeira-Dias ${ }^{93}$ (D),

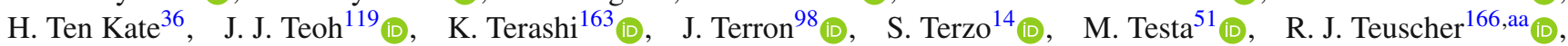

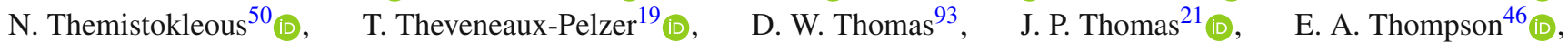

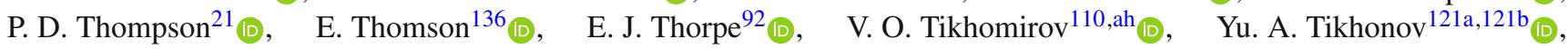
S. Timoshenko ${ }^{111}, \quad$ P. Tipton ${ }^{182}$ (D), S. Tisserant ${ }^{101}$ (D), K. Todome ${ }^{23 \mathrm{a}, 23 \mathrm{~b}}{ }_{\mathbb{D}}, \quad$ S. Todorova-Nova ${ }^{142}$ (D), $\quad$ S. Todt ${ }^{48}$,

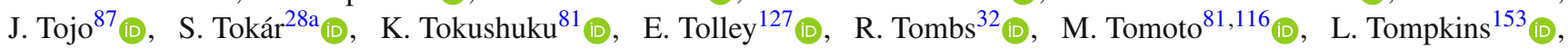

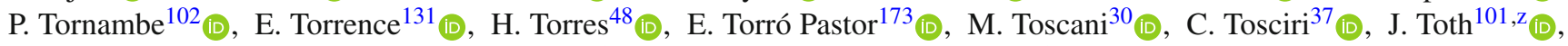

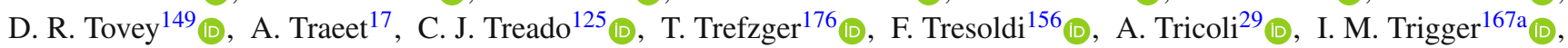
S. Trincaz-Duvoid ${ }^{135}$ (D), D. A. Trischuk ${ }^{174}$ (D), W. Trischuk ${ }^{166}, \quad$ B. $\operatorname{Trocmé}^{58}$ (D), A. Trofymov ${ }^{64}$ (D), C. Troncon ${ }^{68 a_{(D)}}$,

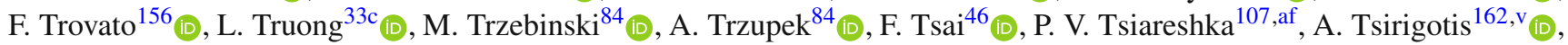
V. Tsiskaridze ${ }^{155}$ (D), E. G. Tskhadadze ${ }^{159 a}$, M. Tsopoulou ${ }^{162}$ (I), I. I. Tsukerman ${ }^{123}$ (D), V. Tsulaia ${ }^{18}$ (D), S. Tsuno ${ }^{81}$ (D),

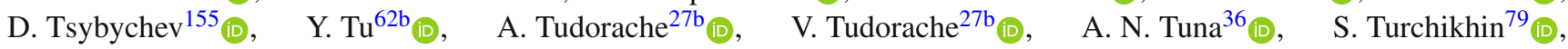

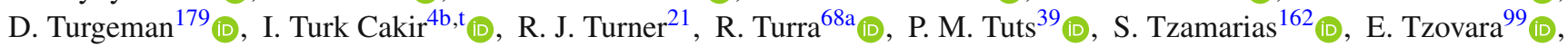

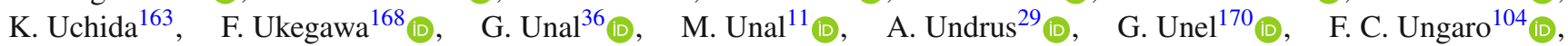

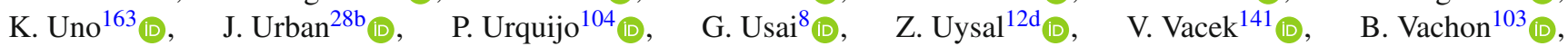

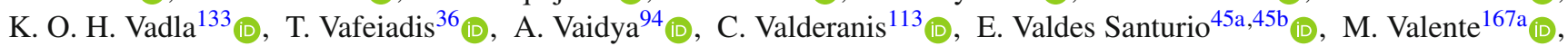

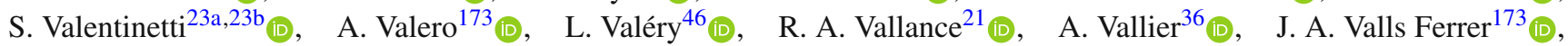

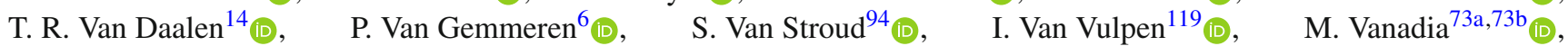

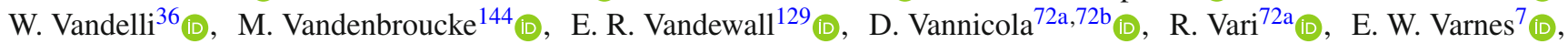

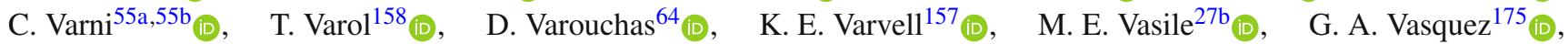
F. Vazeille ${ }^{38}$ (D), D. Vazquez Furelos ${ }^{14}$ (D), T. Vazquez Schroeder ${ }^{36}{ }_{(\mathbb{C})}$, J. Veatch ${ }^{53}$ (D), V. Vecchio ${ }^{100}$ (D), M. J. Veen ${ }^{119}$ (D),

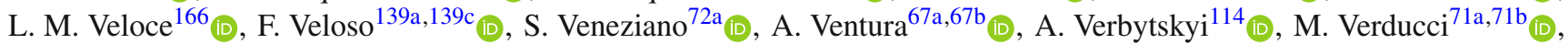
C. Vergis ${ }^{24}$ (D), W. Verkerke ${ }^{119}$ (D), A. T. Vermeulen ${ }^{119}$ (D), J. C. Vermeulen ${ }^{119}$ (D), C. Vernieri ${ }^{153}$ (D), P. J. Verschuuren ${ }^{93}$ (D),

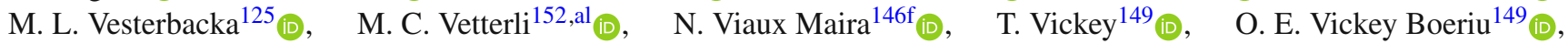

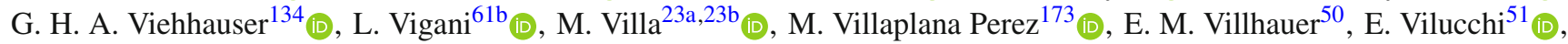

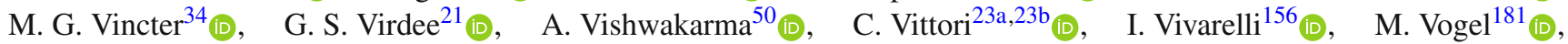

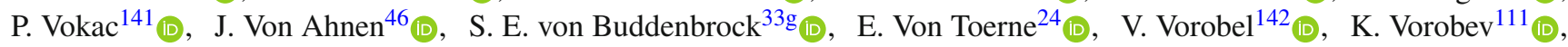

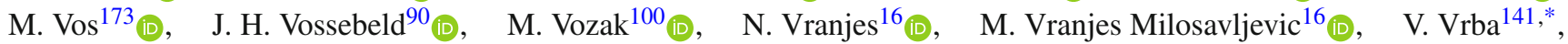
M. Vreeswijk ${ }^{119}$ (D) N. K. Vu ${ }^{101}$ (D), R. Vuillermet ${ }^{36}$ (D) I. Vukotic ${ }^{37}$ (D), S. Wada ${ }^{168}$ (D) , C. Wagner ${ }^{102}$, P. Wagner ${ }^{24}$ (D), W. Wagner ${ }^{181}$ (D) , S. Wahdan ${ }^{181}$ (D) H. Wahlberg ${ }^{88}$ (D), R. Wakasa ${ }^{168}$ (D) , V. M. Walbrecht ${ }^{114}$ (D) J. Walder ${ }^{143}$ (ID), R. Walker ${ }^{113}$ (D),

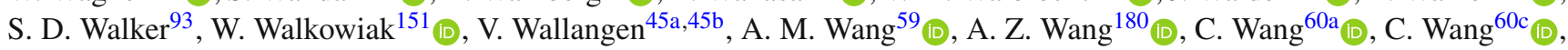

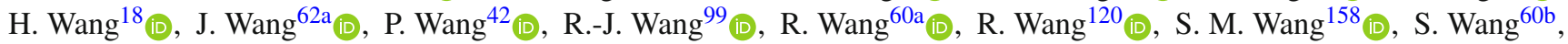




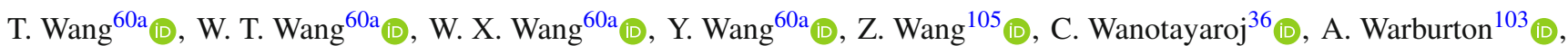

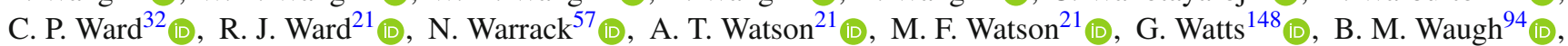

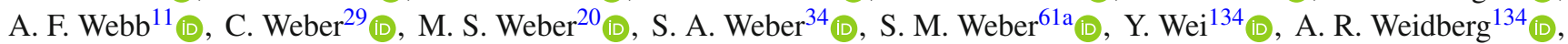
J. Weingarten ${ }^{47}$ (D), M. Weirich ${ }^{99}$ (D), C. Weiser ${ }^{52}$ (D), P. S. Wells ${ }^{36}$ (D) T. Wenaus ${ }^{29}$ (D), B. Wendland ${ }^{47}$ (D), T. Wengler ${ }^{36}$ (D),

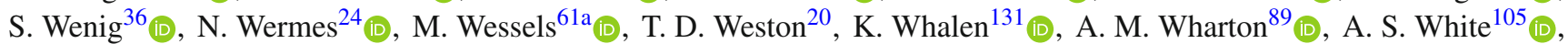

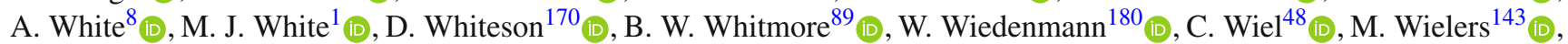
N. Wieseotte ${ }^{99}$, C. Wiglesworth ${ }^{40}$ (D) L. A. M. Wiik-Fuchs ${ }^{52}$ (D) H. G. Wilkens ${ }^{36}$ (D) L. J. Wilkins ${ }^{93}$ (D), D. M. Williams ${ }^{39}{ }^{(D)}$, H. H. Williams ${ }^{136}$, S. Williams ${ }^{32}$ (D) S. Willocq ${ }^{102}$ (D) P. J. Windischhofer ${ }^{134}$ (D), I. Wingerter-Seez ${ }^{5}$ (D) E. Winkels ${ }^{156}$ (D), F. Winklmeier ${ }^{131}$ (D), B. T. Winter ${ }^{52}$ (D), M. Wittgen ${ }^{153}$, M. Wobisch ${ }^{95}$ (D), A. Wolf ${ }^{99}$ (D), R. Wölker ${ }^{134}$ (I) J. Wollrath ${ }^{52}$,

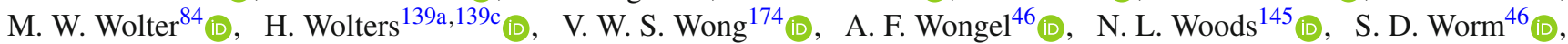

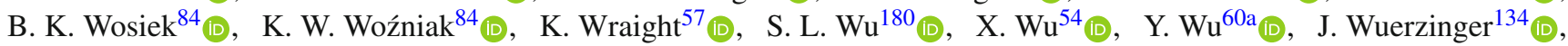

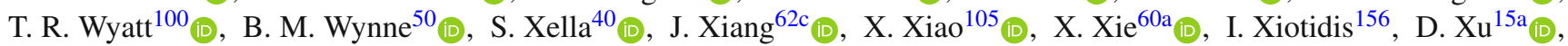

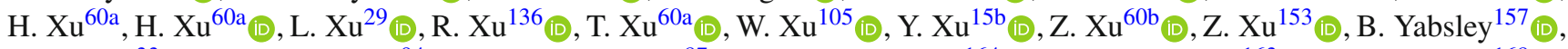

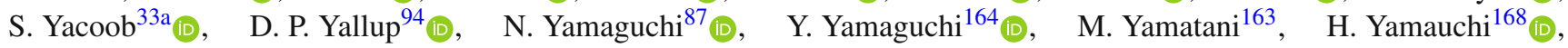

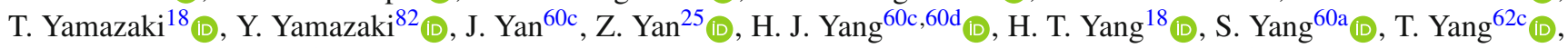

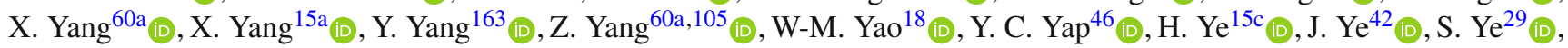
I. Yeletskikh ${ }^{79}$ (D), M. R. Yexley ${ }^{89}$ (D), P. Yin ${ }^{39}$ (D), K. Yorita ${ }^{178}$ (D), K. Yoshihara ${ }^{78}$ (D) C. J. S. Young ${ }^{36}$ (D), C. Young ${ }^{153}$ (D),

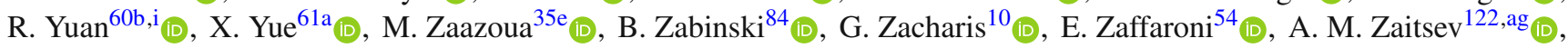

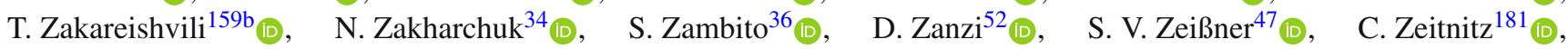

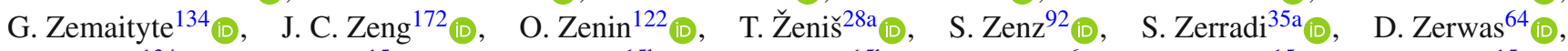

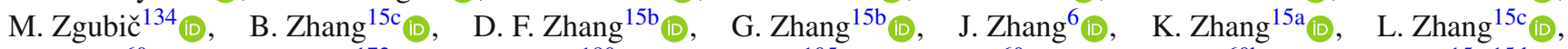

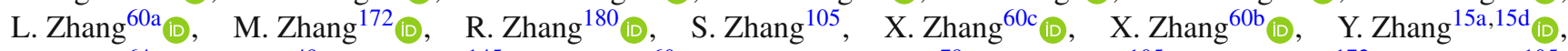
Z. Zhang ${ }^{64}$ (D) P. Zhao ${ }^{49}$ (D) Y. Zhao ${ }^{145}$ (D) Z. Zhao ${ }^{60 a}$ (D), A. Zhemchugov ${ }^{79}$ (D) Z. Zheng ${ }^{105}$ (D), D. Zhong ${ }^{172}$ (D), B. Zhou ${ }^{105}$,

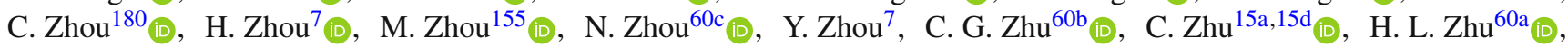

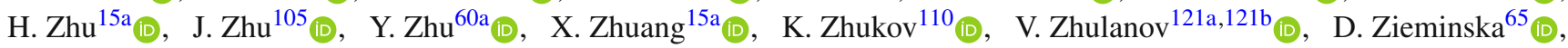

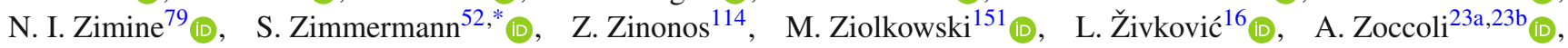

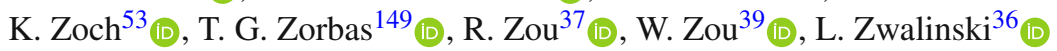

${ }^{1}$ Department of Physics, University of Adelaide, Adelaide, Australia

${ }^{2}$ Physics Department, SUNY Albany, Albany, NY, USA

${ }^{3}$ Department of Physics, University of Alberta, Edmonton, AB, Canada

4 (a) Department of Physics, Ankara University, Ankara, Turkey; ${ }^{(b)}$ Istanbul Aydin University, Application and Research

Center for Advanced Studies, Istanbul, Turkey; ${ }^{(c)}$ Division of Physics, TOBB University of Economics and Technology,

Ankara, Turkey

${ }^{5}$ LAPP, Univ. Savoie Mont Blanc, CNRS/IN2P3, Annecy, France

${ }^{6}$ High Energy Physics Division, Argonne National Laboratory, Argonne, IL, USA

${ }^{7}$ Department of Physics, University of Arizona, Tucson, AZ, USA

${ }^{8}$ Department of Physics, University of Texas at Arlington, Arlington, TX, USA

${ }^{9}$ Physics Department, National and Kapodistrian University of Athens, Athens, Greece

${ }^{10}$ Physics Department, National Technical University of Athens, Zografou, Greece

${ }^{11}$ Department of Physics, University of Texas at Austin, Austin, TX, USA

12 (a) Bahcesehir University, Faculty of Engineering and Natural Sciences, Istanbul, Turkey; ${ }^{(b)}$ Istanbul Bilgi University,

Faculty of Engineering and Natural Sciences, Istanbul, Turkey; ${ }^{\left({ }^{c}\right)}$ Department of Physics, Bogazici University, Istanbul, Turkey; ${ }^{(d)}$ Department of Physics Engineering, Gaziantep University, Gaziantep, Turkey; ${ }^{(e)}$ Department of Physics,

Istanbul University, Istanbul, Turkey; ${ }^{(f)}$ Istinye University, Sariyer, Istanbul, Turkey

${ }^{13}$ Institute of Physics, Azerbaijan Academy of Sciences, Baku, Azerbaijan

${ }^{14}$ Institut de Física d'Altes Energies (IFAE), Barcelona Institute of Science and Technology, Barcelona, Spain

15 (a) Institute of High Energy Physics, Chinese Academy of Sciences, Beijing, China; ${ }^{(b)}$ Physics Department, Tsinghua

University, Beijing, China; ${ }^{(c)}$ Department of Physics, Nanjing University, Nanjing, China; ${ }^{(d)}$ University of Chinese

Academy of Science (UCAS), Beijing, China

${ }^{16}$ Institute of Physics, University of Belgrade, Belgrade, Serbia

${ }^{17}$ Department for Physics and Technology, University of Bergen, Bergen, Norway

${ }^{18}$ Physics Division, Lawrence Berkeley National Laboratory and University of California, Berkeley, CA, USA 
${ }^{19}$ Institut für Physik, Humboldt Universität zu Berlin, Berlin, Germany

${ }^{20}$ Albert Einstein Center for Fundamental Physics and Laboratory for High Energy Physics, University of Bern, Bern, Switzerland

${ }^{21}$ School of Physics and Astronomy, University of Birmingham, Birmingham, UK

22 (a) Facultad de Ciencias y Centro de Investigaciónes, Universidad Antonio Nariño, Bogotá, Colombia; ${ }^{(b)}$ Departamento de Física, Universidad Nacional de Colombia, Bogotá, Colombia

23 (a) Dipartimento di Fisica e Astronomia A. Righi, Università di Bologna, Bologna, Italy; ${ }^{\left({ }^{b}\right)}$ INFN Sezione di Bologna, Bologna, Italy

${ }^{24}$ Physikalisches Institut, Universität Bonn, Bonn, Germany

${ }^{25}$ Department of Physics, Boston University, Boston, MA, USA

${ }^{26}$ Department of Physics, Brandeis University, Waltham, MA, USA

27 (a) Transilvania University of Brasov, Brasov, Romania; ${ }^{(b)}$ Horia Hulubei National Institute of Physics and Nuclear Engineering, Bucharest, Romania; ${ }^{(c)}$ Department of Physics, Alexandru Ioan Cuza University of Iasi, Iasi, Romania; ${ }^{(d)}$ Physics Department, National Institute for Research and Development of Isotopic and Molecular Technologies, Cluj-Napoca, Romania; ${ }^{\left({ }^{e}\right)}$ University Politehnica Bucharest, Bucharest, Romania; ${ }^{(\mathrm{f})}$ West University in Timisoara, Timisoara, Romania

28 (a) Faculty of Mathematics, Physics and Informatics, Comenius University, Bratislava, Slovak Republic; (b) Department of Subnuclear Physics, Institute of Experimental Physics of the Slovak Academy of Sciences, Kosice, Slovak Republic

${ }^{29}$ Physics Department, Brookhaven National Laboratory, Upton, NY, USA

${ }^{30}$ Departamento de Física (FCEN) and IFIBA, Universidad de Buenos Aires and CONICET, Buenos Aires, Argentina

${ }^{31}$ California State University, Long Beach, CA, USA

${ }^{32}$ Cavendish Laboratory, University of Cambridge, Cambridge, UK

33 (a) Department of Physics, University of Cape Town, Cape Town, South Africa; ${ }^{(b)}$ iThemba Labs, Western Cape, South Africa; ${ }^{(c)}$ Department of Mechanical Engineering Science, University of Johannesburg, Johannesburg, South Africa; ${ }^{(d)}$ National Institute of Physics, University of the Philippines, Diliman, Philippines; ${ }^{(e)}$ Department of Physics, University of South Africa, Pretoria, South Africa; (f) University of Zululand, KwaDlangezwa,

South Africa; ${ }^{(\mathrm{g})}$ School of Physics, University of the Witwatersrand, Johannesburg, South Africa

${ }^{34}$ Department of Physics, Carleton University, Ottawa, ON, Canada

35 (a) Faculté des Sciences Ain Chock, Réseau Universitaire de Physique des Hautes Energies - Université Hassan II, Casablanca, Morocco; ${ }^{(b)}$ Faculté des Sciences, Université Ibn-Tofail, Kénitra, Morocco; ${ }^{(c)}$ Faculté des Sciences Semlalia, Université Cadi Ayyad, LPHEA-Marrakech, Marrakech, Morocco; ${ }^{(d)}$ LPMR, Faculté des Sciences, Université Mohamed Premier, Oujda, Morocco; ${ }^{(\mathrm{e})}$ Faculté des sciences, Université Mohammed V, Rabat, Morocco; ${ }^{(\mathrm{f})}$ Mohammed VI Polytechnic University, Ben Guerir, Morocco

${ }^{36}$ CERN, Geneva, Switzerland

${ }^{37}$ Enrico Fermi Institute, University of Chicago, Chicago, IL, USA

${ }^{38}$ LPC, Université Clermont Auvergne, CNRS/IN2P3, Clermont-Ferrand, France

${ }^{39}$ Nevis Laboratory, Columbia University, Irvington, NY, USA

${ }^{40}$ Niels Bohr Institute, University of Copenhagen, Copenhagen, Denmark

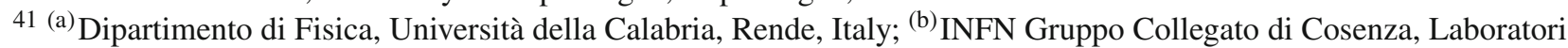
Nazionali di Frascati, Frascati, Italy

42 Physics Department, Southern Methodist University, Dallas, TX, USA

${ }^{43}$ Physics Department, University of Texas at Dallas, Richardson, TX, USA

${ }^{44}$ National Centre for Scientific Research "Demokritos", Agia Paraskevi, Greece

45 (a) Department of Physics, Stockholm University, Stockholm, Sweden; ${ }^{(b)}$ Oskar Klein Centre, Stockholm, Sweden

${ }^{46}$ Deutsches Elektronen-Synchrotron DESY, Hamburg and Zeuthen, Germany

${ }^{47}$ Lehrstuhl für Experimentelle Physik IV, Technische Universität Dortmund, Dortmund, Germany

${ }^{48}$ Institut für Kern- und Teilchenphysik, Technische Universität Dresden, Dresden, Germany

${ }^{49}$ Department of Physics, Duke University, Durham, NC, USA

${ }^{50}$ SUPA-School of Physics and Astronomy, University of Edinburgh, Edinburgh, UK

${ }^{51}$ INFN e Laboratori Nazionali di Frascati, Frascati, Italy

${ }^{52}$ Physikalisches Institut, Albert-Ludwigs-Universität Freiburg, Freiburg, Germany

${ }^{53}$ II. Physikalisches Institut, Georg-August-Universität Göttingen, Göttingen, Germany

${ }^{54}$ Département de Physique Nucléaire et Corpusculaire, Université de Genève, Geneva, Switzerland 
55 (a) Dipartimento di Fisica, Università di Genova, Genoa, Italy; ${ }^{(b)}$ INFN Sezione di Genova, Genoa, Italy

${ }^{56}$ II. Physikalisches Institut, Justus-Liebig-Universität Giessen, Giessen, Germany

${ }^{57}$ SUPA-School of Physics and Astronomy, University of Glasgow, Glasgow, UK

${ }^{58}$ LPSC, Université Grenoble Alpes, CNRS/IN2P3, Grenoble INP, Grenoble, France

${ }^{59}$ Laboratory for Particle Physics and Cosmology, Harvard University, Cambridge, MA, USA

60 (a) Department of Modern Physics and State Key Laboratory of Particle Detection and Electronics, University of Science and Technology of China, Hefei, China; ${ }^{(b)}$ Institute of Frontier and Interdisciplinary Science and Key Laboratory of Particle Physics and Particle Irradiation (MOE), Shandong University, Qingdao, China; ${ }^{(c)}$ School of Physics and

Astronomy, Key Laboratory for Particle Astrophysics and Cosmology (MOE), SKLPPC, Shanghai Jiao Tong University, Shanghai, China; ${ }^{(d)}$ Tsung-Dao Lee Institute, Shanghai, China

61 (a) Kirchhoff-Institut für Physik, Ruprecht-Karls-Universität Heidelberg, Heidelberg, Germany; ${ }^{(b)}$ Physikalisches Institut, Ruprecht-Karls-Universität Heidelberg, Heidelberg, Germany

62 (a) Department of Physics, Chinese University of Hong Kong, Shatin, N.T., Hong Kong, China; ${ }^{(b)}$ Department of Physics, University of Hong Kong, Hong Kong, China; ${ }^{(c)}$ Department of Physics and Institute for Advanced Study, Hong Kong

University of Science and Technology, Clear Water Bay, Kowloon, Hong Kong, China

${ }^{63}$ Department of Physics, National Tsing Hua University, Hsinchu, Taiwan

${ }^{64}$ IJCLab, Université Paris-Saclay, CNRS/IN2P3, 91405 Orsay, France

${ }^{65}$ Department of Physics, Indiana University, Bloomington, IN, USA

66 (a) INFN Gruppo Collegato di Udine, Sezione di Trieste, Udine, Italy; ${ }^{(b)}$ ICTP, Trieste, Italy; ${ }^{(c)}$ Dipartimento Politecnico di Ingegneria e Architettura, Università di Udine, Udine, Italy

67 (a) INFN Sezione di Lecce, Lecce, Italy; ${ }^{\left({ }^{b}\right)}$ Dipartimento di Matematica e Fisica, Università del Salento, Lecce, Italy

68 (a) INFN Sezione di Milano, Milan, Italy; ${ }^{(b)}$ Dipartimento di Fisica, Università di Milano, Milan, Italy

69 (a) INFN Sezione di Napoli, Naples, Italy; ${ }^{(b)}$ Dipartimento di Fisica, Università di Napoli, Naples, Italy

70 (a) INFN Sezione di Pavia, Pavia, Italy; ${ }^{(b)}$ Dipartimento di Fisica, Università di Pavia, Pavia, Italy

71 (a) INFN Sezione di Pisa, Pisa, Italy; ${ }^{\text {(b) }}$ Dipartimento di Fisica E. Fermi, Università di Pisa, Pisa, Italy

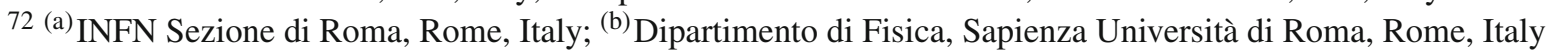

73 (a) INFN Sezione di Roma Tor Vergata, Rome, Italy; ${ }^{(b)}$ Dipartimento di Fisica, Università di Roma Tor Vergata, Rome, Italy

74 (a) INFN Sezione di Roma Tre, Rome, Italy; ${ }^{(b)}$ Dipartimento di Matematica e Fisica, Università Roma Tre, Rome, Italy

75 (a) INFN-TIFPA, Povo, Italy; ${ }^{\text {(b) }}$ Università degli Studi di Trento, Trento, Italy

${ }^{76}$ Institut für Astro- und Teilchenphysik, Leopold-Franzens-Universität, Innsbruck, Austria

${ }^{77}$ University of Iowa, Iowa City, IA, USA

${ }^{78}$ Department of Physics and Astronomy, Iowa State University, Ames, IA, USA

${ }^{79}$ Joint Institute for Nuclear Research, Dubna, Russia

80 (a) Departamento de Engenharia Elétrica, Universidade Federal de Juiz de Fora (UFJF), Juiz de Fora,

Brazil; ${ }^{(b)}$ Universidade Federal do Rio De Janeiro COPPE/EE/IF, Rio de Janeiro, Brazil; ${ }^{(c)}$ Instituto de Física,

Universidade de São Paulo, São Paulo, Brazil; ${ }^{(d)}$ Rio de Janeiro State University, Rio de Janeiro, Brazil

${ }^{81}$ KEK, High Energy Accelerator Research Organization, Tsukuba, Japan

${ }^{82}$ Graduate School of Science, Kobe University, Kobe, Japan

83 (a) AGH University of Science and Technology, Faculty of Physics and Applied Computer Science, Krakow,

Poland; ${ }^{(b)}$ Marian Smoluchowski Institute of Physics, Jagiellonian University, Krakow, Poland

${ }^{84}$ Institute of Nuclear Physics Polish Academy of Sciences, Krakow, Poland

${ }^{85}$ Faculty of Science, Kyoto University, Kyoto, Japan

${ }^{86}$ Kyoto University of Education, Kyoto, Japan

${ }^{87}$ Research Center for Advanced Particle Physics and Department of Physics, Kyushu University, Fukuoka, Japan

${ }^{88}$ Instituto de Física La Plata, Universidad Nacional de La Plata and CONICET, La Plata, Argentina

${ }^{89}$ Physics Department, Lancaster University, Lancaster, UK

${ }^{90}$ Oliver Lodge Laboratory, University of Liverpool, Liverpool, UK

${ }^{91}$ Department of Experimental Particle Physics, Jožef Stefan Institute and Department of Physics, University of Ljubljana, Ljubljana, Slovenia

92 School of Physics and Astronomy, Queen Mary University of London, London, UK

${ }^{93}$ Department of Physics, Royal Holloway University of London, Egham, UK

${ }^{94}$ Department of Physics and Astronomy, University College London, London, UK 
${ }^{95}$ Louisiana Tech University, Ruston, LA, USA

${ }^{96}$ Fysiska institutionen, Lunds universitet, Lund, Sweden

${ }^{97}$ Centre de Calcul de l'Institut National de Physique Nucléaire et de Physique des Particules (IN2P3), Villeurbanne, France

${ }^{98}$ Departamento de Física Teorica C-15 and CIAFF, Universidad Autónoma de Madrid, Madrid, Spain

${ }^{99}$ Institut für Physik, Universität Mainz, Mainz, Germany

${ }^{100}$ School of Physics and Astronomy, University of Manchester, Manchester, UK

${ }^{101}$ CPPM, Aix-Marseille Université, CNRS/IN2P3, Marseille, France

102 Department of Physics, University of Massachusetts, Amherst, MA, USA

${ }^{103}$ Department of Physics, McGill University, Montreal, QC, Canada

${ }^{104}$ School of Physics, University of Melbourne, Melbourne, VIC, Australia

105 Department of Physics, University of Michigan, Ann Arbor, MI, USA

${ }^{106}$ Department of Physics and Astronomy, Michigan State University, East Lansing, MI, USA

${ }^{107}$ B.I. Stepanov Institute of Physics, National Academy of Sciences of Belarus, Minsk, Belarus

108 Research Institute for Nuclear Problems of Byelorussian State University, Minsk, Belarus

109 Group of Particle Physics, University of Montreal, Montreal, QC, Canada

${ }^{110}$ P.N. Lebedev Physical Institute of the Russian Academy of Sciences, Moscow, Russia

${ }^{111}$ National Research Nuclear University MEPhI, Moscow, Russia

112 D.V. Skobeltsyn Institute of Nuclear Physics, M.V. Lomonosov Moscow State University, Moscow, Russia

${ }^{113}$ Fakultät für Physik, Ludwig-Maximilians-Universität München, Munich, Germany

${ }^{114}$ Max-Planck-Institut für Physik (Werner-Heisenberg-Institut), Munich, Germany

115 Nagasaki Institute of Applied Science, Nagasaki, Japan

116 Graduate School of Science and Kobayashi-Maskawa Institute, Nagoya University, Nagoya, Japan

117 Department of Physics and Astronomy, University of New Mexico, Albuquerque, NM, USA

118 Institute for Mathematics, Astrophysics and Particle Physics, Radboud University/Nikhef, Nijmegen, The Netherlands

${ }^{119}$ Nikhef National Institute for Subatomic Physics and University of Amsterdam, Amsterdam, The Netherlands

${ }^{120}$ Department of Physics, Northern Illinois University, DeKalb, IL, USA

121 (a) Budker Institute of Nuclear Physics and NSU, SB RAS, Novosibirsk, Russia; ${ }^{(b)}$ Novosibirsk State University,

Novosibirsk, Russia

122 Institute for High Energy Physics of the National Research Centre Kurchatov Institute, Protvino, Russia

${ }^{123}$ Institute for Theoretical and Experimental Physics named by A.I. Alikhanov of National Research Centre "Kurchatov Institute", Moscow, Russia

124 (a) New York University Abu Dhabi, Abu Dhabi, United Arab Emirates; ${ }^{(b)}$ United Arab Emirates University, Al Ain, United Arab Emirates; ${ }^{(c)}$ University of Sharjah, Sharjah, United Arab Emirates

125 Department of Physics, New York University, New York, NY, USA

${ }^{126}$ Ochanomizu University, Otsuka, Bunkyo-ku, Tokyo, Japan

127 Ohio State University, Columbus, OH, USA

${ }^{128}$ Homer L. Dodge Department of Physics and Astronomy, University of Oklahoma, Norman, OK, USA

${ }^{129}$ Department of Physics, Oklahoma State University, Stillwater, OK, USA

${ }^{130}$ Palacký University, Joint Laboratory of Optics, Olomouc, Czech Republic

${ }^{131}$ Institute for Fundamental Science, University of Oregon, Eugene, OR, USA

132 Graduate School of Science, Osaka University, Osaka, Japan

${ }^{133}$ Department of Physics, University of Oslo, Oslo, Norway

${ }^{134}$ Department of Physics, Oxford University, Oxford, UK

${ }^{135}$ LPNHE, Sorbonne Université, Université de Paris, CNRS/IN2P3, Paris, France

${ }^{136}$ Department of Physics, University of Pennsylvania, Philadelphia, PA, USA

${ }^{137}$ Konstantinov Nuclear Physics Institute of National Research Centre "Kurchatov Institute", PNPI, St. Petersburg, Russia

138 Department of Physics and Astronomy, University of Pittsburgh, Pittsburgh, PA, USA

139 (a) Laboratório de Instrumentação e Física Experimental de Partículas - LIP, Lisbon, Portugal; ${ }^{\left({ }^{b}\right)}$ Departamento de Física, Faculdade de Ciências, Universidade de Lisboa, Lisbon, Portugal; ${ }^{(c)}$ Departamento de Física, Universidade de Coimbra, Coimbra, Portugal; ${ }^{(d)}$ Centro de Física Nuclear da Universidade de Lisboa, Lisbon, Portugal; ${ }^{(e)}$ Departamento de Física, Universidade do Minho, Braga, Portugal; ${ }^{(f)}$ Departamento de Física Teórica y del Cosmos, Universidad de Granada, 
Granada, Spain; ${ }^{(\mathrm{g})}$ Dep Física and CEFITEC of Faculdade de Ciências e Tecnologia, Universidade Nova de Lisboa,

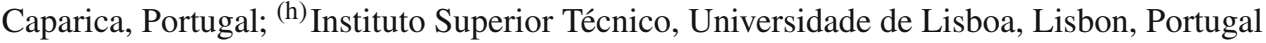

${ }^{140}$ Institute of Physics of the Czech Academy of Sciences, Prague, Czech Republic

${ }^{141}$ Czech Technical University in Prague, Prague, Czech Republic

142 Faculty of Mathematics and Physics, Charles University, Prague, Czech Republic

${ }^{143}$ Particle Physics Department, Rutherford Appleton Laboratory, Didcot, UK

${ }^{144}$ IRFU, CEA, Université Paris-Saclay, Gif-sur-Yvette, France

${ }^{145}$ Santa Cruz Institute for Particle Physics, University of California Santa Cruz, Santa Cruz, CA, USA

146 (a) Departamento de Física, Pontificia Universidad Católica de Chile, Santiago, Chile; ${ }^{(b)}$ Millennium Institute for

Subatomic Physics at High Energy Frontier (SAPHIR), Santiago, Chile; ${ }^{(c)}$ Universidad de la Serena, La Serena,

Chile; (d) Department of Physics, Universidad Andres Bello, Santiago, Chile; ${ }^{(e)}$ Instituto de Alta Investigación,

Universidad de Tarapacá, Arica, Chile; ${ }^{(\mathfrak{f})}$ Departamento de Física, Universidad Técnica Federico Santa María,

Valparaíso, Chile

147 Universidade Federal de São João del Rei (UFSJ), São João del Rei, Brazil

148 Department of Physics, University of Washington, Seattle, WA, USA

${ }^{149}$ Department of Physics and Astronomy, University of Sheffield, Sheffield, UK

${ }^{150}$ Department of Physics, Shinshu University, Nagano, Japan

${ }^{151}$ Department Physik, Universität Siegen, Siegen, Germany

152 Department of Physics, Simon Fraser University, Burnaby, BC, Canada

${ }^{153}$ SLAC National Accelerator Laboratory, Stanford, CA, USA

${ }^{154}$ Department of Physics, Royal Institute of Technology, Stockholm, Sweden

${ }^{155}$ Departments of Physics and Astronomy, Stony Brook University, Stony Brook, NY, USA

156 Department of Physics and Astronomy, University of Sussex, Brighton, UK

157 School of Physics, University of Sydney, Sydney, Australia

${ }^{158}$ Institute of Physics, Academia Sinica, Taipei, Taiwan

159 (a) E. Andronikashvili Institute of Physics, Iv. Javakhishvili Tbilisi State University, Tbilisi, Georgia; ${ }^{(b)}$ High Energy

Physics Institute, Tbilisi State University, Tbilisi, Georgia

160 Department of Physics, Technion, Israel Institute of Technology, Haifa, Israel

${ }^{161}$ Raymond and Beverly Sackler School of Physics and Astronomy, Tel Aviv University, Tel Aviv, Israel

162 Department of Physics, Aristotle University of Thessaloniki, Thessaloniki, Greece

${ }^{163}$ International Center for Elementary Particle Physics and Department of Physics, University of Tokyo, Tokyo, Japan

${ }^{164}$ Department of Physics, Tokyo Institute of Technology, Tokyo, Japan

165 Tomsk State University, Tomsk, Russia

166 Department of Physics, University of Toronto, Toronto, ON, Canada

167 (a) TRIUMF, Vancouver, BC, Canada; ${ }^{\text {(b) }}$ Department of Physics and Astronomy, York University, Toronto, ON, Canada

${ }^{168}$ Division of Physics and Tomonaga Center for the History of the Universe, Faculty of Pure and Applied Sciences,

University of Tsukuba, Tsukuba, Japan

169 Department of Physics and Astronomy, Tufts University, Medford, MA, USA

${ }^{170}$ Department of Physics and Astronomy, University of California Irvine, Irvine, CA, USA

${ }^{171}$ Department of Physics and Astronomy, University of Uppsala, Uppsala, Sweden

172 Department of Physics, University of Illinois, Urbana, IL, USA

${ }^{173}$ Instituto de Física Corpuscular (IFIC), Centro Mixto Universidad de Valencia - CSIC, Valencia, Spain

${ }^{174}$ Department of Physics, University of British Columbia, Vancouver, BC, Canada

175 Department of Physics and Astronomy, University of Victoria, Victoria, BC, Canada

${ }^{176}$ Fakultät für Physik und Astronomie, Julius-Maximilians-Universität Würzburg, Würzburg, Germany

${ }^{177}$ Department of Physics, University of Warwick, Coventry, UK

${ }^{178}$ Waseda University, Tokyo, Japan

${ }^{179}$ Department of Particle Physics and Astrophysics, Weizmann Institute of Science, Rehovot, Israel

${ }^{180}$ Department of Physics, University of Wisconsin, Madison, WI, USA

${ }^{181}$ Fakultät für Mathematik und Naturwissenschaften, Fachgruppe Physik, Bergische Universität Wuppertal, Wuppertal, Germany

${ }^{182}$ Department of Physics, Yale University, New Haven, CT, USA 
${ }^{a}$ Also at Borough of Manhattan Community College, City University of New York, New York, NY, USA

${ }^{\mathrm{b}}$ Also at Center for High Energy Physics, Peking University, Beijing, China

${ }^{c}$ Also at Centro Studi e Ricerche Enrico Fermi, Rome, Italy

${ }^{\mathrm{d}}$ Also at CERN, Geneva, Switzerland

e Also at CPPM, Aix-Marseille Université, CNRS/IN2P3, Marseille, France

${ }^{\mathrm{f}}$ Also at Département de Physique Nucléaire et Corpusculaire, Université de Genève, Geneva, Switzerland

g Also at Departament de Fisica de la Universitat Autonoma de Barcelona, Barcelona, Spain

${ }^{\mathrm{h}}$ Also at Department of Financial and Management Engineering, University of the Aegean, Chios, Greece

${ }^{\mathrm{i}}$ Also at Department of Physics and Astronomy, Michigan State University, East Lansing, MI, USA

${ }^{\mathrm{j}}$ Also at Department of Physics and Astronomy, University of Louisville, Louisville, KY, USA

${ }^{k}$ Also at Department of Physics, Ben Gurion University of the Negev, Beer Sheva, Israel

${ }^{1}$ Also at Department of Physics, California State University, East Bay, USA

${ }^{m}$ Also at Department of Physics, California State University, Fresno, USA

${ }^{\mathrm{n}}$ Also at Department of Physics, California State University, Sacramento, USA

${ }^{\circ}$ Also at Department of Physics, King's College London, London, UK

p Also at Department of Physics, St. Petersburg State Polytechnical University, St. Petersburg, Russia

${ }^{\mathrm{q}}$ Also at Department of Physics, University of Fribourg, Fribourg, Switzerland

${ }^{\mathrm{r}}$ Also at Dipartimento di Matematica, Informatica e Fisica, Università di Udine, Udine, Italy

${ }^{s}$ Also at Faculty of Physics, M.V. Lomonosov Moscow State University, Moscow, Russia

${ }^{t}$ Also at Giresun University, Faculty of Engineering, Giresun, Turkey

u Also at Graduate School of Science, Osaka University, Osaka, Japan

${ }^{v}$ Also at Hellenic Open University, Patras, Greece

${ }^{w}$ Also at Institucio Catalana de Recerca i Estudis Avancats, ICREA, Barcelona, Spain

x Also at Institut für Experimentalphysik, Universität Hamburg, Hamburg, Germany

${ }^{y}$ Also at Institute for Nuclear Research and Nuclear Energy (INRNE) of the Bulgarian Academy of Sciences, Sofia, Bulgaria

${ }^{\mathrm{z}}$ Also at Institute for Particle and Nuclear Physics, Wigner Research Centre for Physics, Budapest, Hungary

aa Also at Institute of Particle Physics (IPP), Toronto, Canada

${ }^{a b}$ Also at Institute of Physics, Azerbaijan Academy of Sciences, Baku, Azerbaijan

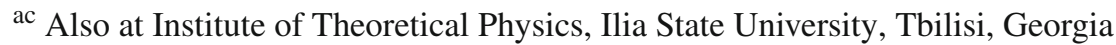

${ }^{\text {ad }}$ Also at Instituto de Fisica Teorica, IFT-UAM/CSIC, Madrid, Spain

ae Also at Department of Physics, Istanbul University, Istanbul, Turkey

${ }^{a f}$ Also at Joint Institute for Nuclear Research, Dubna, Russia

ag Also at Moscow Institute of Physics and Technology State University, Dolgoprudny, Russia

ah Also at National Research Nuclear University MEPhI, Moscow, Russia

ai Also at Physics Department, An-Najah National University, Nablus, Palestine

aj Also at Physikalisches Institut, Albert-Ludwigs-Universität Freiburg, Freiburg, Germany

${ }^{a k}$ Also at The City College of New York, New York, NY, USA

${ }^{\text {al }}$ Also at TRIUMF, Vancouver, BC, Canada

am Also at Universita di Napoli Parthenope, Naples, Italy

${ }^{\text {an }}$ Also at University of Chinese Academy of Sciences (UCAS), Beijing, China

* Deceased 